\title{
A multicentred randomised controlled trial of a primary care-based cognitive behavioural programme for low back pain. The Back Skills Training (BeST) trial
}

SE Lamb, R Lall, Z Hansen,

E Castelnuovo, EJ Withers, V Nichols,

F Griffiths, R Potter, A Szczepura

and $M$ Underwood, on behalf of the BeST trial group

Health Technology Assessment NIHR HTA programme www.hta.ac.uk 


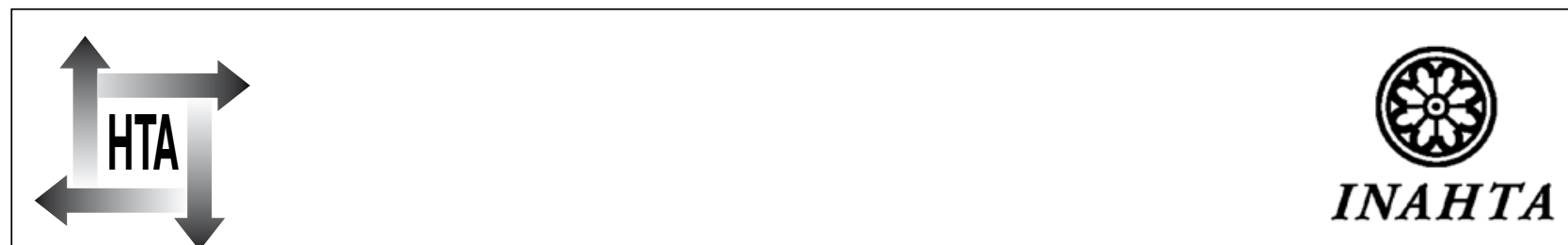

How to obtain copies of this and other HTA programme reports

An electronic version of this title, in Adobe Acrobat format, is available for downloading free of charge for personal use from the HTA website (www.hta.ac.uk).A fully searchable DVD is also available (see below).

Printed copies of HTA journal series issues cost $£ 20$ each (post and packing free in the UK) to both public and private sector purchasers from our despatch agents.

Non-UK purchasers will have to pay a small fee for post and packing. For European countries the cost is $£ 2$ per issue and for the rest of the world $€ 3$ per issue.

How to order:

- fax (with credit card details)

- post (with credit card details or cheque)

- phone during office hours (credit card only).

Additionally the HTA website allows you to either print out your order or download a blank order form.

\section{Contact details are as follows:}

Synergie UK (HTA Department)

Digital House, The Loddon Centre

Wade Road

Basingstoke

Hants RG24 8QW
Email: orders@hta.ac.uk

Tel: 08458124000 - ask for 'HTA Payment Services' (out-of-hours answer-phone service)

Fax: 0845812400 I - put 'HTA Order' on the fax header

\section{Payment methods}

Paying by cheque

If you pay by cheque, the cheque must be in pounds sterling, made payable to University of Southampton and drawn on a bank with a UK address.

Paying by credit card

You can order using your credit card by phone, fax or post.

\section{Subscriptions}

NHS libraries can subscribe free of charge. Public libraries can subscribe at a reduced cost of $£ 100$ for each volume (normally comprising 40-50 titles). The commercial subscription rate is $£ 400$ per volume (addresses within the UK) and $£ 600$ per volume (addresses outside the UK). Please see our website for details. Subscriptions can be purchased only for the current or forthcoming volume.

\section{How do I get a copy of HTA on DVD?}

Please use the form on the HTA website (www.hta.ac.uk/htacd/index.shtml). HTA on DVD is currently free of charge worldwide.

The website also provides information about the HTA programme and lists the membership of the various committees. 


\title{
A multicentred randomised controlled trial of a primary care-based cognitive behavioural programme for low back pain. The Back Skills Training (BeST) trial
}

\author{
SE Lamb,'* R Lall,' Z Hansen,' \\ E Castelnuovo, ${ }^{2}$ EJ Withers,' $\vee$ Nichols,' \\ F Griffiths,' R Potter,' A Szczepura ${ }^{3}$ \\ and M Underwood,' on behalf of the \\ BeST trial group
}

'Warwick Clinical Trials Unit, Warwick Medical School,

University of Warwick, Coventry UK

${ }^{2}$ Centre for Health Economics, University of York, York, UK

${ }^{3}$ Health Sciences Research Institute, Warwick Medical School,

University of Warwick, Coventry, UK

*Corresponding author

Declared competing interests of authors: none

Published August 2010

DOI: $10.3310 / \mathrm{hta} 14410$

This report should be referenced as follows:

Lamb SE, Lall R, Hansen Z, Castelnuovo E, Withers EJ, Nichols V, et al. A multicentred randomised controlled trial of a primary care-based cognitive behavioural programme for low back pain. The Back Skills Training (BeST) trial. Health Technol Assess 20 10; I 4(4I).

Health Technology Assessment is indexed and abstracted in Index Medicus/MEDLINE, Excerpta Medica/EMBASE, Science Citation Index Expanded (SciSearch ${ }^{\circledR}$ ) and Current Contents ${ }^{\circledR} /$ Clinical Medicine. 


\section{NIHR Health Technology Assessment programme}

$T$ he Health Technology Assessment (HTA) programme, part of the National Institute for Health Research (NIHR), was set up in 1993. It produces high-quality research information on the effectiveness, costs and broader impact of health technologies for those who use, manage and provide care in the NHS. 'Health technologies' are broadly defined as all interventions used to promote health, prevent and treat disease, and improve rehabilitation and long-term care.

The research findings from the HTA programme directly influence decision-making bodies such as the National Institute for Health and Clinical Excellence (NICE) and the National Screening Committee (NSC). HTA findings also help to improve the quality of clinical practice in the NHS indirectly in that they form a key component of the 'National Knowledge Service'.

The HTA programme is needs led in that it fills gaps in the evidence needed by the NHS. There are three routes to the start of projects.

First is the commissioned route. Suggestions for research are actively sought from people working in the NHS, from the public and consumer groups and from professional bodies such as royal colleges and NHS trusts. These suggestions are carefully prioritised by panels of independent experts (including NHS service users). The HTA programme then commissions the research by competitive tender.

Second, the HTA programme provides grants for clinical trials for researchers who identify research questions. These are assessed for importance to patients and the NHS, and scientific rigour.

Third, through its Technology Assessment Report (TAR) call-off contract, the HTA programme commissions bespoke reports, principally for NICE, but also for other policy-makers. TARs bring together evidence on the value of specific technologies.

Some HTA research projects, including TARs, may take only months, others need several years. They can cost from as little as $£ 40,000$ to over $£ 1$ million, and may involve synthesising existing evidence, undertaking a trial, or other research collecting new data to answer a research problem.

The final reports from HTA projects are peer reviewed by a number of independent expert referees before publication in the widely read journal series Health Technology Assessment.

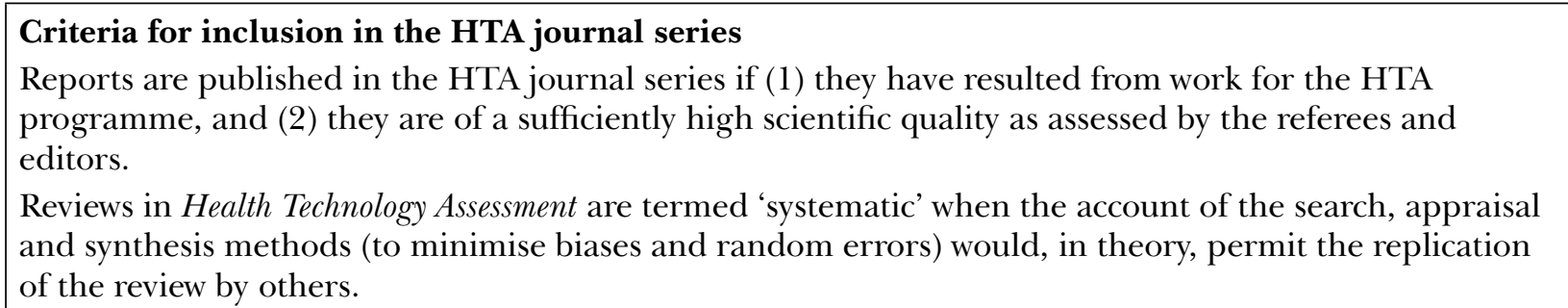

The research reported in this issue of the journal was commissioned by the HTA programme as project number $01 / 75 / 01$. The contractual start date was in October 2003. The draft report began editorial review in January 2009 and was accepted for publication in August 2009. As the funder, by devising a commissioning brief, the HTA programme specified the research question and study design. The authors have been wholly responsible for all data collection, analysis and interpretation, and for writing up their work. The HTA editors and publisher have tried to ensure the accuracy of the authors' report and would like to thank the referees for their constructive comments on the draft document. However, they do not accept liability for damages or losses arising from material published in this report.

The views expressed in this publication are those of the authors and not necessarily those of the HTA programme or the Department of Health.

Editor-in-Chief: $\quad$ Professor Tom Walley CBE

Series Editors: $\quad$ Dr Martin Ashton-Key, Dr Aileen Clarke, Professor Chris Hyde,

Editorial Contact: $\quad$ edit@southampton.ac.uk

ISSN I366-5278

(C) 2010 Queen's Printer and Controller of HMSO

This journal is a member of and subscribes to the principles of the Committee on Publication Ethics (COPE) (http://www.publicationethics.org/). This journal may be freely reproduced for the purposes of private research and study and may be included in professional journals provided that suitable acknowledgement is made and the reproduction is not associated with any form of advertising.

Applications for commercial reproduction should be addressed to: NETSCC, Health Technology Assessment, Alpha House, University of

Southampton Science Park, Southampton SOI6 7NS, UK.

Published by Prepress Projects Ltd, Perth, Scotland (www.prepress-projects.co.uk), on behalf of NETSCC, HTA.

Printed on acid-free paper in the UK by Henry Ling Ltd, The Dorset Press, Dorchester. 


\title{
Abstract
}

\section{A multicentred randomised controlled trial of a primary care-based cognitive behavioural programme for low back pain. The Back Skills Training (BeST) trial}

\author{
SE Lamb,'* R Lall,' Z Hansen,' E Castelnuovo, ${ }^{2}$ EJ Withers,' V Nichols, ' \\ F Griffiths,' R Potter,' A Szczepura ${ }^{3}$ and M Underwood,' on behalf of \\ the BeST trial group
}

\author{
'Warwick Clinical Trials Unit, Warwick Medical School, University of Warwick, Coventry, UK \\ ${ }^{2}$ Centre for Health Economics, University of York, York, UK \\ ${ }^{3}$ Health Sciences Research Institute, Warwick Medical School, University of Warwick, \\ Coventry, UK
}

*Corresponding author

Objectives: To estimate the clinical effectiveness of active management (AM) in general practice versus AM plus a group-based, professionally led cognitive behavioural approach (CBA) for subacute and chronic low back pain (LBP) and to measure the cost of each strategy over a period of 12 months and estimate costeffectiveness.

Design: Pragmatic multicentred randomised controlled trial with investigator-blinded assessment of outcomes.

Setting: Fifty-six general practices from seven English regions.

Participants: People with subacute and chronic LBP who were experiencing symptoms that were at least moderately troublesome.

Interventions: Participants were randomised (in a ratio of $2: 1$ ) to receive either $A M+C B A$ or $A M$ alone. Main outcome measures: Primary outcomes were the Roland Morris Disability Questionnaire (RMQ) and the Modified Von Korff Scale (MVK), which measure LBP and disability. Secondary outcomes included mental and physical health-related quality of life (Short Form 12-item health survey), health status, fear avoidance beliefs and pain self-efficacy. Costutility of CBA was considered from both the UK NHS perspective and a broader health-care perspective, including both NHS costs and costs of privately purchased goods and services related to LBP. Qualityadjusted life-years (QALYs) were calculated from the five-item EuroQoL.

Results: Between April 2005 and April 2007, 70I participants were randomised: 233 to $A M$ and 468 to $A M+C B A$. Of these, 420 were female. The mean age of participants was 54 years and mean baseline RMQ was 8.7. Outcome data were obtained for $85 \%$ of participants at 12 months. Benefits were seen across a range of outcome measures in favour of CBA with no evidence of group or therapist effects. CBA resulted in at least twice as much improvement as AM. Mean additional improvement in the CBA arm was I.I [ $95 \%$ confidence interval $(\mathrm{Cl}) 0.4$ to I.7], I.4 (95\% Cl 0.7 to 2.1 ) and I.3 (95\% Cl 0.6 to 2.1 ) change points in the RMQ at 3, 6 and 12 months respectively. Additional improvement in MVK pain was $6.8(95 \%$ Cl 3.5 to 10.2$), 8.0$ (95\% Cl 4.3 to II.7) and $7.0(95 \%$ $\mathrm{Cl} 3.2$ to 10.7 ) points, and in MVK disability was 4.3 (95\% $\mathrm{Cl} 0.4$ to 8.2 ), 8.1 (95\% $\mathrm{Cl} 4.1$ to 12.0 ) and 8.4 (95\% $\mathrm{Cl} 4.4$ to 12.4 ) points at 3, 6 and 12 months respectively. At 12 months, $60 \%$ of the AM+CBA arm and $31 \%$ of the AM arm reported some or complete recovery. Mean cost of attending a CBA course was $£ 187$ per participant with an additional benefit in QALYs of 0.099 and an additional cost of $£ 178.06$. Incremental cost-effectiveness ratio was $£ \mid 786.00$. Probability of CBA being cost-effective reached $90 \%$ at about $£ 3000$ and remained at that level or above; at a cost-effectiveness threshold of $£ 20,000$, the CBA group had an almost $100 \%$ probability of being considered cost-effective. User perspectives on the acceptability of group treatments were sought through semi-structured interviews. Most were familiar with key messages of AM; most who had attended any group sessions had retained key messages from the sessions and two-thirds talked about a reduction in 
fear avoidance and changes in their behaviour. Group sessions appeared to provide reassurance, lessen isolation and enable participants to learn strategies from each other.

Conclusions: Long-term effectiveness and costeffectiveness of CBA in treating subacute and chronic LBP was shown, making this intervention attractive to patients, clinicians and purchasers. Short-term (3-month) clinical effects were similar to those found in high-quality studies of other therapies and benefits were maintained and increased over the long term (12 months). Cost per QALY was about half that of competing interventions for LBP and because the intervention can be delivered by existing NHS staff following brief training, the back skills training programme could be implemented within the NHS with relative ease.

Trial registration: Current Controlled Trials ISRCTN37807450.

Funding: The National Institute for Health Research Health Technology Assessment programme. 


\section{Contents}

List of abbreviations

vii

Executive summary

I Introduction

Background

Sampling approach and risk factors for the development of chronic low back pain

Current management in the UK

Cognitive behavioural approaches in low back pain

2 Characteristics and description of the intervention

Introduction

Identifying the targets for a cognitive behavioural intervention for low back pain

Framework and structure of the cognitive behavioural intervention

Therapist recruitment and training ..........

Assessment of treatment fidelity

Summary

3 Methods

Aims

Research methods

Treatments

Study procedures

Outcome assessments

Other treatments

Randomisation

Quality assurance

Compliance

Formal approvals

Adverse events

Sample size

Pilot study

Data management

Statistical analysis

Monitoring and time line

4 Results

Participant approach and characteristics of the randomised sample

Description of sample by cluster

Success of randomisation

Flow and characteristics of randomised participants
Missing data and characteristics of the randomised sample 32

Treatments received ................................. 32

Adverse events ...................................... 48

Subgroup analysis ............................... 48

Sensitivity analysis $\ldots \ldots \ldots \ldots \ldots \ldots \ldots \ldots \ldots \ldots . . . \ldots \ldots$

5 Qualitative study ............................... 55

BeST interview study ............................. 55

Description of intervention $\ldots \ldots \ldots \ldots \ldots \ldots \ldots . . . \ldots 6$

The interview ..................................... 56

Data coding and analysis $\ldots \ldots \ldots \ldots \ldots \ldots \ldots \ldots . . \ldots \ldots$

Results ............................................... 58

Conclusion ......................................... 64

6 Economic analysis .............................. 67

Introduction ........................................... 67

Data ............................................... 67

The cost of cognitive behavioural therapy in the randomised controlled study (within-trial analysis) ........................ 70

Methods of analysis ............................. 71

Results ............................................... 75

7 Discussion .......................................... 89

Aims and overview of findings ................. 89

External validity and generalisability of findings 89

Critique of methods and threats to internal validity

How have we determined that this is a clinically important result? ................. 90

Comments on best practice advice ........... 91

Comments on the cognitive behavioural approach tested in BeST ................... 91

Other literature: comparisons of cognitive behavioural therapy with advice interventions in primary care ... 92

Comparison of cognitive behavioural interventions with other physical interventions in primary care

Comparison of other physical treatments with advice only in primary care ......... 93

Cost-effectiveness ................................. 93

Addressing concerns about training and delivery

Other things we have learnt about cognitive behavioural approach interventions

Future research questions 
8

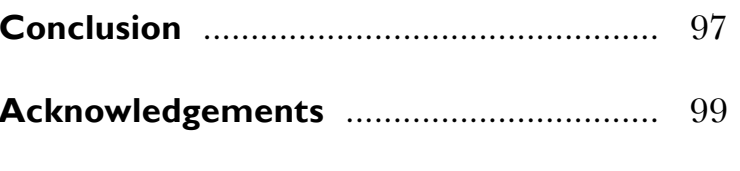

References

101

Appendix I Participant initial approach questionnaire

Appendix 2 First nurse assessment questionnaire

Appendix 3 Temporary exclusion letter

Appendix 4 Nurse randomisation assessment questionnaire

Appendix 5 Consent form

Appendix 6 Baseline questionnaire 131

Appendix 7 Randomisation form 149

Appendix 8 Follow-up questionnaires 151
Appendix 9 Core outcome

questionnaires

Appendix 10 Event notification form

Appendix II Demography and baseline assessments

Appendix 12 Missing data and characteristics of the randomised sample

Appendix 13 Data on employment sickness and benefit status

Appendix 14 Qualitative interview schedule

Appendix 15 Generalised NHS scenario as a secondary analysis

Health Technology Assessment reports published to date

Health Technology Assessment programme 


\section{List of abbreviations}

\begin{tabular}{|c|c|c|c|}
\hline $\mathrm{A} \& \mathrm{E}$ & Accident and Emergency & ICER & incremental cost-effectiveness ratio \\
\hline $\mathrm{AM}$ & active management & ICUR & incremental cost-utility ratio \\
\hline $\mathrm{AM}+\mathrm{CBA}$ & Active Management + Cognitive & ISAP & International Society of Pain \\
\hline ANOVA & $\begin{array}{l}\text { Behavioural Approach } \\
\text { analysis of variance }\end{array}$ & ISRCTN & $\begin{array}{l}\text { International Standard } \\
\text { Randomised Controlled Trial }\end{array}$ \\
\hline ВАВСР & $\begin{array}{l}\text { British Association of Behavioural } \\
\text { and Cognitive Psychotherapies }\end{array}$ & LBP & low back pain \\
\hline BEAM & $\begin{array}{l}\text { Back Pain Exercise and } \\
\text { Manipulation }\end{array}$ & MCID & $\begin{array}{l}\text { minimal clinically important } \\
\text { difference }\end{array}$ \\
\hline $\mathrm{BeST}$ & Back Skills Training & MRC & Medical Research Council \\
\hline $\mathrm{CB}$ & cognitive behaviour & MRI & magnetic resonance imaging \\
\hline CBA & cognitive behavioural approach & MVK & Modified Von Korff Scale \\
\hline CI & confidence interval & NICE & National Institute for Health and \\
\hline CT & computerised tomography & & Cimical excentence \\
\hline DMEC & $\begin{array}{l}\text { Data Monitoring and Ethics } \\
\text { Committee }\end{array}$ & $\begin{array}{l}\text { PCA } \\
\text { PCT }\end{array}$ & $\begin{array}{l}\text { prescription cost analysis } \\
\text { primary-care trust }\end{array}$ \\
\hline EQ-5D & EuroQoL five dimensions & QALY(s) & quality-adjusted life-year(s) \\
\hline FABQ & $\begin{array}{l}\text { Fear Avoidance Beliefs } \\
\text { Questionnaire }\end{array}$ & $\mathrm{RMQ}$ & $\begin{array}{l}\text { Roland Morris Disability } \\
\text { Questionnaire }\end{array}$ \\
\hline GP & general practitioner & SD & standard deviation \\
\hline GPRF & $\begin{array}{l}\text { General Practice Research } \\
\text { Framework }\end{array}$ & SF-12 & Short Form 12-item health survey \\
\hline ICC & intracluster correlation & & \\
\hline \multicolumn{4}{|c|}{$\begin{array}{l}\text { All abbreviations that have been used in this report are listed here unless the abbreviation is well } \\
\text { known (e.g. NHS), or it has been used only once, or it is a non-standard abbreviation used only in } \\
\text { figures/tables/appendices, in which case the abbreviation is defined in the figure legend or in the } \\
\text { notes at the end of the table. }\end{array}$} \\
\hline
\end{tabular}





\section{. \\ Executive summary}

\section{Background}

Low back pain (LBP) is a common and costly problem for which cognitive behavioural approaches may be effective.

\section{Design}

A randomised controlled trial was undertaken with a parallel economic and qualitative evaluation comparing active management (AM) with AM plus group treatment using a cognitive behavioural approach (CBA). We randomised individuals using a stratified unbalanced randomisation $(2: 1$ in favour of the CBA arm).

\section{Setting}

Fifty-six general practices were recruited from seven English regions.

\section{Control intervention}

Primary-care nurses attended a 1-hour training session on the management of LBP, focusing on internationally accepted best practice recommendations for primary care to promote physical activity and analgesia, and to encourage a positive outlook. Nurses were asked to cascade this information within their general practices, and to see each trial participant for an individual advisory session promoting this approach. The advisory session was supplemented with a copy of the The Back Book, which was designed by experts in LBP, to reinforce the messages described above.

\section{Intervention (cognitive behavioural approach) arm}

Physiotherapists, nurses, psychologists and occupational therapists delivered a simple cognitive behavioural formulation that was tailored for LBP, and designed to target unhelpful beliefs about pain and activity, and promote engagement in leisure, physical and occupational activity.
Therapists attended a 2-day training course and were supported with remote mentorship. The intervention was structured and standardised using a treatment manual for both therapists and participants. Each participant attended for an individualised assessment that included goal setting. Thereafter, the CBA intervention was delivered in groups, with approximately eight people starting each cycle. The contents of the group sessions included goal setting, pacing, challenging beliefs, managing pain and improving communication with health professionals. We defined compliance as attending the assessment and at least three of the six group sessions.

\section{Recruitment}

We identified potential participants by searching electronic general practice records, and from direct referrals from general practitioners. Each potential participant went through a two-stage eligibility check to ensure they had at least moderately troublesome back pain present for at least 6 weeks and to exclude those with a serious disorder causing their LBP.

\section{Follow-up}

We collected follow-up data at 3, 6 and 12 months. The primary method of data capture was postal questionnaire. This was supplemented with telephone data collection for individuals who did not return a questionnaire but were happy to provide information.

\section{Clinical outcomes and analysis}

The primary outcomes were the Roland Morris Disability Questionnaire (RMQ) and the Modified Von Korff Scale (MVK), which measure LBP and disability. Secondary outcomes included mental and physical health-related quality of life (Short Form 12-item health survey; SF-12), health status, fear avoidance beliefs and pain self-efficacy. The planned sample size was 700 . We analysed the 
difference in change from baseline scores at each time point, and also analysed these over time to yield a single summary score. We used a linear regression model for the analysis, as the clustering effects (therapist and groups) were found to be non-significant. Models were adjusted for age, sex and baseline covariates. Subgroup analyses were prespecified for fear avoidance beliefs, and the severity and duration of LBP.

\section{Economic analysis}

We considered the cost-utility of the CBA programme from both the UK NHS perspective and a broader health-care perspective. We included all NHS costs needed to deliver the interventions and to provide health care associated with LBP over a 12-month time horizon. For the health-care perspective we included both NHS costs and costs of privately purchased goods and services related to LBP. Quality-adjusted life-years (QALYs) were calculated from the EuroQoL five dimensions. We collected cost data from participant questionnaires. Costs were in UK pounds (£) actualised to 2008 using the Retail Price Index. Discounting was not applied.

\section{Results}

Between April 2005 and April 2007 we randomised 701 participants who provided baseline data; 233 were randomised to best care (AM) and 468 to best care $(\mathrm{AM})+\mathrm{CBA}$. Nearly $60 \%(420 / 701)$ were female, mean age of participants was 54 [standard deviation (SD) 14.9] years and mean baseline RMQ was 8.7 (SD 4.9). Outcome data were obtained for $85 \%$ of participants at 12 months.

Benefits were seen across the range of outcome measures in favour of CBA. There was no evidence of group or therapist effects. Both treatments showed improvements over baseline, but these were of a different magnitude and time course. Overall, CBA resulted in at least twice as much improvement as AM and, for the primary outcomes; improvements were sustained or increased over time. Mean additional improvement in the CBA arm was 1.1 [95\% confidence interval (CI) 0.4 to 1.7 ], 1.4 (95\% CI 0.7 to 2.1$)$ and 1.3 (95\% CI 0.6 to 2.1 ) change points in the RMQ at 3,6 and 12 months respectively. Additional improvement in MVK (pain) was 6.8 (95\% CI 3.5 to 10.2$), 8.0$ (95\% CI 4.3 to 11.7$)$ and 7.0 points (95\% CI 3.2 to 10.7$)$ at 3,6 and 12 months. For
MVK (disability), additional improvements were 4.3 (95\% CI 0.4 to 8.2 ), 8.1 (95\% CI 4.1 to 12.0 ) and 8.4 points (95\% CI 4.4 to 12.4$)$ at 3,6 and 12 months. All differences in the primary outcomes at 6 and 12 months were statistically significant. Differences in physical health-related quality of life and intermediary outcomes were substantial. At 12 months, the treatment effect size was $0.31,0.41$ and 0.45 for the RMQ, MVK and SF-12 physical health scales respectively. At the same time point, $60 \%$ of the CBA arm and $31 \%$ of the AM arm reported some or complete recovery.

\section{Economics}

The mean cost of attending a CBA course was $£ 187$ per participant, which accounted almost entirely for the average difference in NHS costs between the AM and AM+CBA arms $(£ 224.65$ versus $£ 421.52)$. CBA resulted in an additional benefit in QALYs of 0.099 and an additional cost of $£ 178.06$. The incremental cost-effectiveness ratio was $£ 1786.00$. The probability of CBA being cost-effective reached $90 \%$ at about $£ 3000$ and remained at that level or higher above that threshold. At a cost-effectiveness threshold of $£ 20,000$ group CBA had an almost $100 \%$ probability of being considered cost-effective. The cost per QALY was similar in all sensitivity and prespecified subgroup analyses. From the participant perspective CBA resulted, on average, in an additional $£ 130$ of out-of-pocket expenses, increasing cost per QALY to £3093.

\section{Qualitative study}

We explored user perspectives on the acceptability of group treatments and sought insights into how the intervention might work. Semi-structured interviews were completed in a purposive sample of 34 trial participants $(\mathrm{AM}=18, \mathrm{AM}+\mathrm{CBA}=16)$. Almost everyone was familiar with the key messages of the AM approach, although they had not previously received a copy of The Back Book. Most of those who had attended any group sessions had retained key messages from the sessions and twothirds talked about a reduction in fear avoidance and changes in their behaviour. Most also found the exercises helpful and had incorporated exercise into their daily lives. Different individuals reported different strategies included in the CBA package to be helpful. Several people mentioned the importance of the assessment session. Group sessions appear to provide reassurance, to lessen 
isolation and to enable participants to learn strategies from each other.

\section{Conclusions}

This definitive large-scale randomised controlled trial has demonstrated the long-term effectiveness and cost-effectiveness of CBA in treating subacute and chronic LBP. The clinical effectiveness and cost-effectiveness outcomes are likely to make this intervention attractive to patients, clinicians and purchasers. Our short-term (3-month) clinical effects are similar to those found in high-quality studies of other therapies such as manipulation, acupuncture or exercise. Strikingly, and in contrast to many previous studies, the benefits we observed were maintained and increased over the long term (12 months). The intervention is extremely cost-effective from an NHS and a health-care perspective; cost per QALY is less than or about half that of competing interventions for LBP. Finally, because the intervention can be delivered by existing NHS staff following a brief, 2-day training session, the back skills training programme could be implemented into the NHS with relative ease.

\section{Future research questions}

Future research on implementation of the CBA programme will help to ensure that the benefits we found can be translated into a reduction in LBP and associated disability. Further work is needed to examine alternative strategies to delivery, particularly where these improve patient choice and ability to either attend the sessions or gain the cognitive skills and behavioural stimulus embedded in the approach. Some evidence that CBA may also be of help for other musculoskeletal disorders is given by the effects of the package on generalised physical health-related quality of life. Extended follow-up of the BeST cohort may provide additional useful information on the sustainability of clinical effectiveness and cost-effectiveness, and guide the development of brief interventions to help maintain effects over much longer time periods.

\section{Trial registration}

This trial is registered as ISRCTN37807450. 



\section{Chapter I Introduction}

\section{Background}

Low back pain (LBP) is a major public health problem in Western industrialised societies. ${ }^{1}$ In the UK, the annual period prevalence is approximately $37 \% \%^{2,3}$ and LBP is so common that it affects almost everyone at some time during his or her lifetime. ${ }^{4}$ For the majority, LBP follows a recurrent, fluctuating time course, with about $70 \%$ of patients having at least one recurrence within 12 months. ${ }^{5,6}$ Annually around one in three people have an acute bout of LBP, but symptoms resolve quickly and pose no ongoing problem. ${ }^{7} \mathrm{~A}$ significant proportion of people self-manage LBP without consulting the NHS. Estimates suggest that between $7 \%$ and $20 \%$ of the adult population who experience LBP consult a general practitioner $(\mathrm{GP})^{8}$ and this results in 2.6 million additional consultations annually. ${ }^{9}$ Of these people, $75 \%$ have symptoms 1 year later ${ }^{10,11}$ and about $30 \%$ develop persistent disabling LBP.

The direct health-care costs associated with LBP in 1998 were $£ 1628$ million; the majority of this expenditure was on physiotherapy and general practice. ${ }^{1}$ During the years 1994 and 1995, 116 million production days were lost in the UK as the result of LBP, costing an estimated £10,668 million in production and informal care costs. ${ }^{1}$ The majority of these costs are generated by those with the most chronic symptoms, and as a consequence the prevention and amelioration of chronic disabling pain is now the focus of research and clinical activity in this field. ${ }^{12}$

This introduction provides a background to our sampling strategy, risk factors for developing chronic LBP and reasons why cognitive behavioural approaches (CBAs) may be helpful in LBP, an overview of current management of LBP, and the evidence to support CBA at the time the trial was designed.

\section{Sampling approach and risk factors for the development of chronic low back pain}

The population of people who suffer from low back pain is highly diverse. There have been a number of attempts to define different subgroups but none are satisfactory or in widespread use. We focused our trial on non-specific LBP, which is a term used to describe the majority of presentations, i.e. those in which no serious cause (infection, cancer or fracture) for LBP can be identified. The primary focus of our trial was the prevention or amelioration of disabling LBP in those with established (subacute or chronic) symptoms. We defined LBP according to Croft et al. ${ }^{11}$ and used the International Society of Pain (ISAP) definitions of acute, subacute and chronic duration (Table 1). The ISAP provides a simple and widely used definition of back pain syndromes, although this does not include activity limitation.

Considerable advances have been made in identifying risk factors and understanding the processes involved in the development of chronic LBP. ${ }^{14}$ Psychological, social and behavioural risk factors have been found consistently to be stronger predictors of chronic disability than the physical factors associated with the initial onset of pain. ${ }^{15}$ This does not mean that physical symptoms are unimportant; for example, pain radiating down the leg is acknowledged to be a risk factor for developing chronic symptoms. ${ }^{16,17}$ However, a recent prospective study found only a weak association between structural changes and pain and no association with disability or future health care. $^{18}$

Psychological risk factors play an important role in the progression of LBP to chronic disabling LBP. A review of psychological risk factors found that coping strategies, beliefs, distress, depressive mood and somatisation were associated with the development of persisting pain and disability. ${ }^{19,20}$ Of these, 'fear of movement' (which is a negative health belief about the relationship between pain and re-injury such that if I increase my back pain, I am causing further damage, so I should avoid movement), has been that most consistently and strongly associated with the progression to chronic disability. ${ }^{21,22}$ Participation in physical recreational activity is associated with lower LBP and a reduced risk of progression to disability. ${ }^{23}$ There is now a widely held belief that increasing physical activity and/or exercise participation is important 
TABLE I Case definitions used in the Back Skills Training (BeST) trial

Low back pain - pain of musculoskeletal origin in the area bounded by the 12 th rib and below by the gluteal folds (Croft et al. 1998)"

Acute pain - lasting 6 weeks or less

Subacute pain - lasting 6-12 weeks

Chronic pain - lasting more than 3 months (International Association for the Study of Pain, Merskey et al. 1979) ${ }^{13}$

in managing LBP. ${ }^{24}$ Physical activity is usually characterised as a behavioural factor.

Social factors are also important. A systematic review of social risk factors identified low social support in the workplace, heavy manual work and low job satisfaction as strong risk factors for the occurrence of chronic back pain. ${ }^{25}$ More recent studies have also found strong correlations between chronic back pain and low socioeconomic status, educational level, work satisfaction and female gender. ${ }^{6,26,27}$

Epidemiological evidence suggests that interventions that address this range of psychological, behavioural and social risk factors offer hope in reducing the burden of LBP. Recent clinical guidelines ${ }^{6,26}$ stress the importance of assessing psychosocial factors and emotional distress, and suggest that such assessments might assist in targeting intervention more appropriately. Cognitive behavioural approaches encompass a range of interventions that aim to directly change behaviour using models of learning, and to indirectly change behaviour by changing beliefs and behavioural risk factors However, it is broadly acknowledged that there is insufficient evidence to determine the optimal methods of assessment and intervention and that large-scale evaluation is required.

\section{Current management in the UK}

The majority of LBP is managed in primary care, with a small number of people referred to secondary care, often only for a single consultation and with little consistency or rationale. ${ }^{28}$ About $9 \%$ of the population with LBP attend for physiotherapy treatment, usually after primary-care consultation. ${ }^{1}$ Chiropractic and osteopathy are used less often. ${ }^{1}$

Since 2000, there has been a major change in the approach to managing LBP in primary care known as the active management (AM) strategy, which forms the core of all international guidelines. ${ }^{29-31}$ The AM strategy discourages bed rest for LBP and instead advocates physical activity. ${ }^{10}$ Appropriate medication is encouraged, although many classes of pain medication have been shown to have limited long-term benefits and are recommended most often for the management of acute LBP. ${ }^{32,33}$ An information booklet called The Back Book ${ }^{34}$ has facilitated implementation of the AM guidelines in the UK. The Back Book was designed to challenge negative beliefs and behaviours, rather than merely to impart factual information. A randomised controlled trial evaluating The Back Book showed a positive effect on patients' beliefs and clinical outcomes, maintained at 1 year, for those with both acute and recurrent back pain..$^{25,35}$

A 2007 systematic review ${ }^{36}$ evaluating the effectiveness of advice for the management of LBP compared advice offered to acute, subacute and chronic LBP patients. The review suggests that advice to stay active is sufficient for acute LBP patients and could be more widely implemented in practice. There is uncertainty about the management of both subacute and chronic LBP. No conclusions could be drawn from the evidence base about the optimal method of advice, and although there are suggestions in the literature that additional treatments may be beneficial, differences in case definitions of LBP and the treatments tested make it difficult to draw substantial conclusions about what type of advice is optimal for various presentations of LBP.

Unlike most guidance, and at the time of planning this trial, most UK guidelines tentatively recommended early referral to physical treatments, including acupuncture, spinal manipulation and exercise. ${ }^{1}$ The evidence for these treatments has strengthened during the lifetime of the Back Skills Training (BeST) trial. More recent studies have raised some doubt about the effectiveness of traditional physiotherapy approaches to LBP; for example a comparison of routine physiotherapy with a single session of advice for LBP found no 
difference in treatment outcomes. ${ }^{37}$ An Australian study of participants with subacute LBP, found physiotherapist-directed exercise and advice were each slightly more effective than placebo, with greatest effectiveness when combined. ${ }^{38}$ A Cochrane review concludes that exercise therapy appears to be slightly effective at decreasing pain and improving function in adults with chronic LBP $^{39}$ A meta-analysis of the effectiveness of spinal manipulation therapy for LBP concluded that there is no evidence to suggest that manipulation is superior to other standard treatments for acute $\mathrm{LBP}^{40}$ However, a recent large UK trial comparing manipulation with exercise and a combination of manipulation and exercise found small mediumterm benefits in each treatment arm, and greater benefits if treatments were combined. ${ }^{24}$

We selected the active management strategy and The Back Book as the intervention for the control arm of the trial, as these were recommended as best practice strategies within UK guidance at the time of starting and during the conduct of the trial.

\section{Cognitive behavioural approaches in low back pain}

The CBA includes a range of therapies deriving from cognitive and behavioural psychological models that aim to teach an individual to tackle their problems using specific psychological and practical skills. $^{41}$

Cognitive behavioural approaches for managing LBP were first introduced in the UK as part of inpatient pain management programmes for those with very chronic/severe LBP. Intensive (> 100 hours of therapy) multidisciplinary biopsychosocial rehabilitation programmes were found to improve pain and function for patients in the secondary-care setting. ${ }^{42}$ Systematic reviews suggest that the type of CB treatments for chronic LBP ( $>12$ weeks) that could be delivered in primary care appear to have short-term benefits but, possibly, no sustained long-term benefits. ${ }^{43}$

Although there has been no formal systematic review, trials of CBA in populations with acute and subacute LBP report a mixed picture, including some improvement in disability. ${ }^{44-46}$

Although some trials pointed to the potential for CBA in treatment of subacute and chronic LBP, there was a mixed picture of results. Few programmes found sustained benefits. Differences in results may be attributable to poor research design, but more likely they are the result of variable adherence to the principles of CBA and differences in how the programmes are delivered. These include the amount of contact time, level of expertise, components included in programmes and method of delivery. Indications are that the important attributes of effective interventions are ensuring that the health-care professionals who deliver the interventions are able to elicit psychosocial risk factors, implementing a CB framework that results in modification of beliefs as well as behaviours (as opposed to delivering skills alone), and delivering a treatment that is credible to patients. ${ }^{47,48}$

The aim of this study was therefore to develop and test a group-based CBA intervention that could be delivered within the UK NHS and that could be accessed from primary care. We carried out a definitive randomised controlled trial with a parallel economic and qualitative study that would provide a comprehensive evaluation of CBA as a treatment for subacute and chronic LBP, and provide the NHS with evidence to support decision-making in this area of clinical practice. 



\section{Chapter 2}

\section{Characteristics and description of the intervention}

\section{Introduction}

A detailed description of the theoretical basis of the intervention, as well as dose and mode of delivery is an essential step in the reporting of randomised trials of complex interventions. ${ }^{49}$ Our approach was to draw together the essential elements of a CBA, ensuring that the intervention was consistent with the principles of CB therapy, and that we targeted health behaviours and beliefs that are broadly accepted as being on the causal pathway between LBP and disability. The intervention was developed by systematically reviewing experimental and observational literature, and linking the results of these reviews into a CB framework. We also considered the optimal delivery method to balance clinical effectiveness and cost-effectiveness.

The CB model states that the way a person thinks about their problem will produce emotions, including associated physical sensations, which then drive behaviour. ${ }^{50}$ Often, the behaviour will inadvertently maintain the thoughts or beliefs, so creating a maintenance or vicious cycle effect. The following sections provide a rationale for the risk factors selected as treatment targets for the CBA.

\section{Identifying the targets for a cognitive behavioural intervention for low back pain}

The key modifiable risk factors appear to be psychological and behavioural factors that have a mediating effect on activity levels. Psychological constructs, including catastrophising, passive coping, fear avoidance and depression lead to decreased activity levels or, for some, overactivity. These changes in activity levels are implicated in the development of chronic disabling LBP through a pathway of deconditioning, and worsening pain. We specified the targets of the CB intervention:

- $\quad$ to increase activity levels

- to manage periods of overactivity
- to specifically address catastrophising and fear avoidance

- to improve coping skills.

There is consistent evidence across various reviews that coping skills, catastrophising and fear avoidance are key factors in the progression of acute LBP to chronic disability. Distress has also been identified as an important risk factor; ${ }^{51}$ however, it is difficult to define distress separately from the other psychological constructs and $\operatorname{mood} .6,18,52$

Many studies have pointed to the importance of coping strategies and beliefs held by patients. ${ }^{19,53}$ A sense of personal control and self-efficacy are associated with active coping strategies (e.g. taking exercise). A lack of personal control and feelings of helplessness are associated with passive (maladaptive) coping strategies such as rest and catastrophising. ${ }^{54-57}$

The strong link between beliefs predicting behaviour has been shown in two studies. ${ }^{3,58} \mathrm{~A}$ reduction in patients' belief that they were disabled and that increased pain signified harm and the need to restrict activity, was strongly associated with a reduction in pain behaviours, physical disability and depression. These beliefs are commonly referred to as 'catastrophic beliefs' and lead to avoidance of the feared activity or pain. This behaviour has been labelled 'fear avoidance' and has been consistently and strongly associated with the progression of acute LBP to disability. ${ }^{21,22,59-61}$ In addition, catastrophising is associated with hypervigilance for symptoms, which increases pain perception. ${ }^{62,63}$ Interventions that have used CBA to target catastrophising and fear avoidance behaviours appear to reduce disability in the short term. $^{64}$

At the other end of the activity level spectrum, there are people who increase their activity levels in response to pain. ${ }^{65}$ This overactivity has been linked to mood and to unhelpful beliefs ${ }^{66}$ and can lead to poor control over pain and subsequent activity. 
Depression is associated with the risk of developing chronic LBP, ${ }^{67}$ with a hypothesised pathway of apathy, demotivation and low mood resulting in decreased activity and poor outcome. Exercise is effective in the management of depression ${ }^{68}$ and has the potential to reverse this cycle. ${ }^{69}$

Pain intensity has been positively and consistently associated with a poor outcome. ${ }^{70}$ However, pain intensity is not independent of other psychological constructs such as catastrophising. ${ }^{71}$ Education on pain mechanisms appears effective in changing beliefs and improving physical functioning. ${ }^{72}$

Low levels of physical activity have been shown to correlate with future episodes of persistent LBP. ${ }^{23,73,74}$ In addition, a significant proportion of the LBP population will reduce their activity levels in response to developing LBP. ${ }^{75}$ The resultant 'deconditioning or disuse syndrome' describes the physical decline in strength, mobility, endurance and coordination that is postulated to contribute to ongoing pain. ${ }^{76}$

Several systematic reviews have investigated the efficacy of exercises in the treatment of LBP and found modest improvements in pain and function. ${ }^{77-78}$ Hayden et al. ${ }^{79}$ went on to perform a meta-regression to identify features of the exercise programmes associated with successful outcomes. They concluded that the most effective exercises were conducted as part of an individualised supervised programme and included stretching and strengthening exercises. Although counterintuitive, particularly for those suffering LBP, exercise does not increase recurrence of pain. ${ }^{80-82}$ There have been relatively few interventions that have focused on improving general or functional activities. ${ }^{78}$

The intervention did not intend to target social factors that are not modifiable within the context of a primary care-based intervention (e.g. educational level, work-related risk factors, job satisfaction). Models of pain based on disc degeneration, posture, injury, weight and leg-length discrepancy were excluded because there is little evidence to support a relationship between these factors and chronic LBP. ${ }^{83,84}$

\section{Framework and structure of the cognitive behavioural intervention}

The British Association of Behavioural and definitions of CB therapy ${ }^{41}$ and suggests that the following components are essential:

- The intervention should be delivered collaboratively, i.e. intervention draws on the expertise of therapist and client and direction of treatment is determined jointly.

- The intervention should use a goal-oriented approach, i.e. treatment is centred around achieving the client's goals.

- The intervention should use the CB model, i.e. intervention links thoughts, feelings and behaviours.

- The intervention should explore unhelpful beliefs through CB questioning techniques, e.g. the use of open questions to help the client consider alternative ways of thinking.

- The intervention should focus on the development of specific psychological and practical skills to enable the client to tackle problems independently, e.g. problem-solving and relaxation skills.

- The intervention should use homework to achieve skill development, e.g. practising skills discussed during treatment sessions.

We designed an intervention comprising an assessment followed by six group sessions based on these principles. The CB model we developed to underpin the interventions is shown in Figure 1. The figure depicts the concept that back pain leads to altered activity and associated physical changes, which in turn leads to maintenance or worsening of pain. These relationships are mediated by unhelpful thoughts and feelings. The shaded boxes indicate the key skills developed by the BeST intervention, and their hypothesised mode of action.

\section{Group format}

The intervention was designed for group delivery. A review of outcomes in individual versus group $\mathrm{CB}$ therapy found little difference in efficacy. ${ }^{85}$ Group treatment has the potential to maximise costeffectiveness $^{86}$ as well as providing additional nonspecific effects. These benefits include participant modelling of helpful coping strategies, support and increased opportunity for developing problemsolving, a key skill in self-management. ${ }^{87}$

\section{Assessment session}

We chose an initial one-to-one assessment session of up to 90 minutes, which would allow the therapists to better understand an individual's problems and negotiate goals. This assessment also 


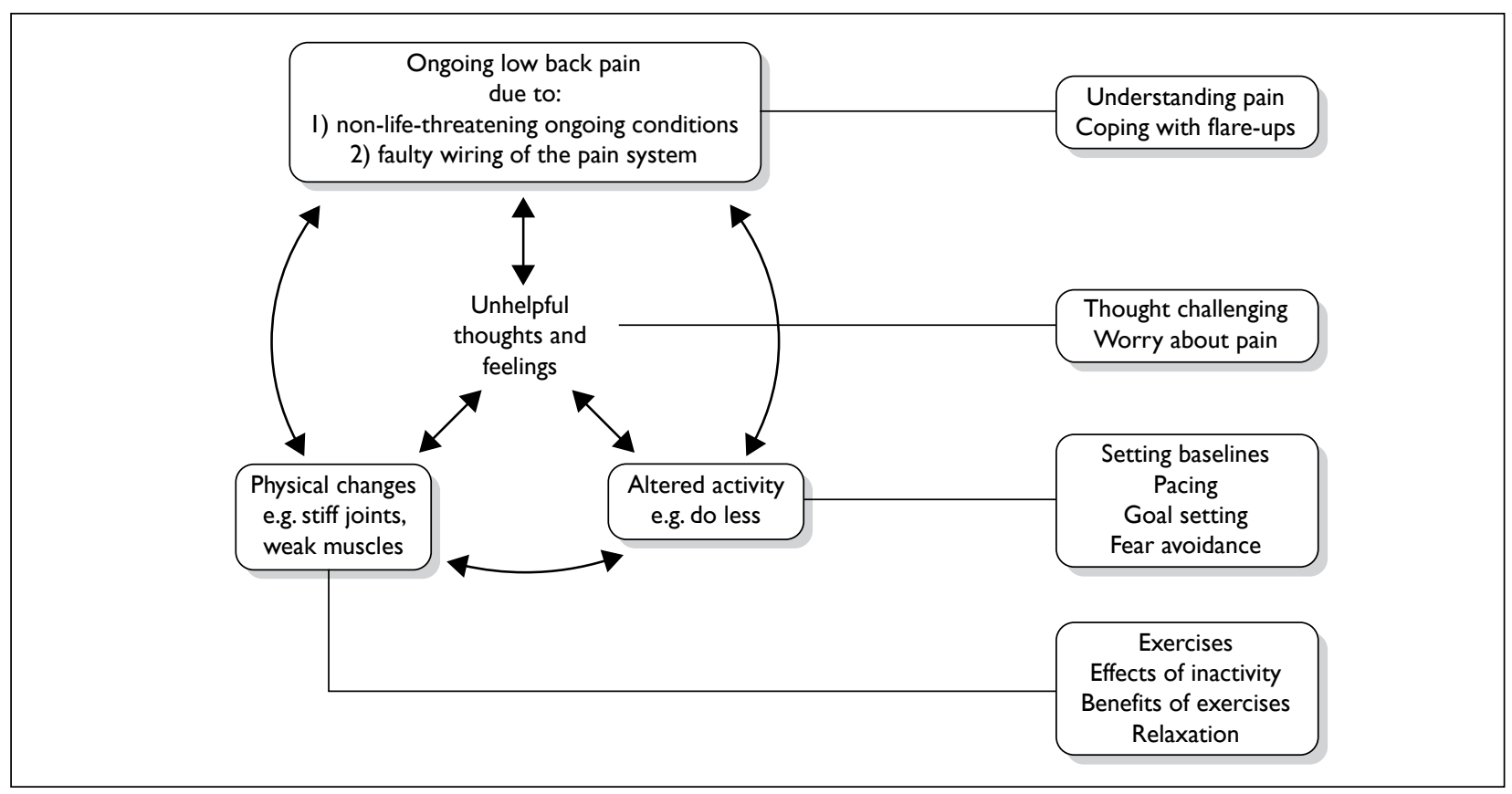

FIGURE I Integrated model of LBP. Text in boxes indicates how sessions target components.

included the negotiation of a simple home exercise programme as we considered promotion of physical activity central to the intervention (this could be as little as one activity). These exercises were checked and progressed on at least one occasion during the group sessions. Goal setting for the programme was an important product of the assessment. Goals were set collaboratively in the assessment session and reviewed as a group in the second session.

\section{Group session length}

Group sessions were of 90 minutes' duration, with a frequency of once per week for 6 weeks (i.e. a total of 10.5 hours including the assessment). The length of the sessions and overall programme was designed to facilitate attendance while ensuring sufficient time to develop skills. A small study on pain management programmes found no difference in outcomes between group or individual treatment and programmes that were 15, 30 or 60 hours in duration. ${ }^{88}$ However, very brief interventions of less than 1 hour were found not to be effective. ${ }^{89}$

\section{Group session content}

The content of each session was prespecified and documented in the therapist's manual. Details of the session contents are given in Table 2. Therapists were requested not only to cover the content of the sessions but also to respond flexibly to participants' needs as necessary.

\section{Group structure}

Each session was structured in line with standard CB therapy approaches to include agenda setting, topics and homework review. The work of the previous session was reviewed briefly at the beginning of each session, and all sessions included a 10-minute break midway through to allow participants to move around and exercise (if they wished to). Each session started with agenda setting, which included asking participants what they wanted to cover in the sessions. Homework was reviewed at some point during each session to allow for group problem-solving.

Each session focused on one or more of the components of the CB model shown in Figure 1. We used simple CB maintenance models for fear avoidance and hypervigilance to provide a structure and rationale for the skills being taught in the sessions (Figures 2 and 3). These provide a conceptual framework for the relationship between thoughts, feelings and behaviours which could be communicated to participants, and form the foundation of discussions to enable problemsolving to break vicious cycles.

We prespecified a benchmark in attendance to define those who we believed had been compliant with the intervention, and those who had not. This threshold was attendance at the assessment session and at least three of the six group sessions. Although not evidence based, we hypothesised that this was the minimum number of sessions required 
TABLE 2 Details of CBA sessions

\section{Session number}

I $\quad 2 \quad 3 \quad 4 \quad 5 \quad 6$

\section{Assessment}

History taking, including current problems and eliciting beliefs on LBP and activity

Collaborative goal setting with plan to start activity goal

Exercises chosen collaboratively from options with level negotiated

Exercises practised and progression discussed

\section{Understanding pain}

Group activity to demonstrate hurt does not equal harm

Current thinking on causes of long-term pain explained

Discussion on group's experience of alternative treatments for LBP with

reference to research evidence and need to self-manage

\section{Benefits of exercise}

Discussion of physical impact of inactivity or altered activity and how changes impact on pain (disuse syndrome)

Discussion on effects of activity/exercise

Introduction to LBP model (Figure I)

\section{Pain fluctuations}

Overactivity/underactivity cycle explained

Use of pacing

Group problem-solving for overactivity, e.g. gardening

\section{Working out starting point for exercises or activities}

How to use baseline setting

\section{How to set goals}

SMART system used to break down an example goal

Feedback from group on how progressing with goals from assessment

Group problem-solving problems with goals

\section{Unhelpful thoughts and feelings}

Styles of unhelpful thinking discussed, including catastrophising

Link with unhelpful behaviours

Identifying unhelpful thoughts

Group problem-solving for challenging unhelpful thoughts

\section{Restarting activities or hobbies}

Discussion on activities commonly avoided in LBP

Fear avoidance cycle (Figure 2)

Group problem-solving out of cycle

Development of specific goals relating to restarting activities

\section{When pain worries us}

Effect of attention to pain explored through group activity

Hypervigilance cycle (Figure 3) used to link unhelpful thoughts and behaviours

Group problem-solving out of cycle

Discussion on the use of medication/distraction/alternating activities

\section{Coping with flare-ups}

Discussion on causes of flare-ups

Plan of what to do in and out of flare-ups

Revision of topics over previous sessions and questions 


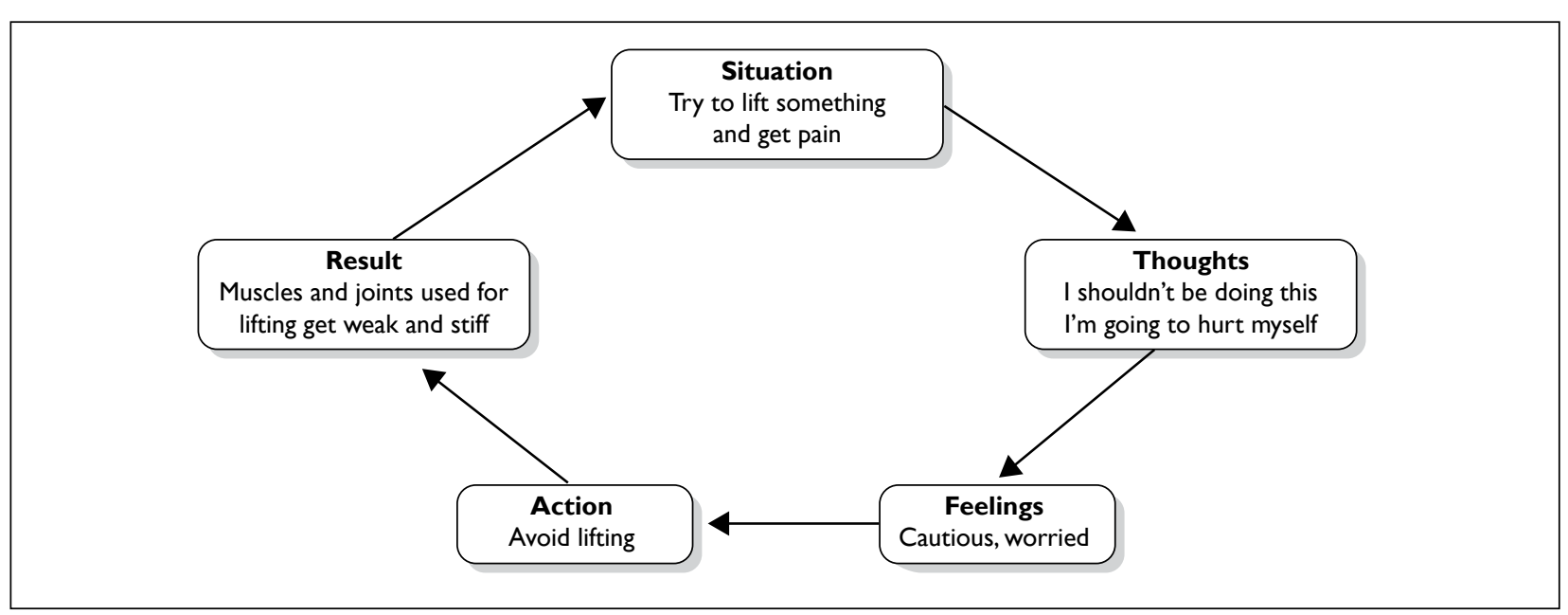

FIGURE 2 Fear avoidance cycle used in BeST session 4.

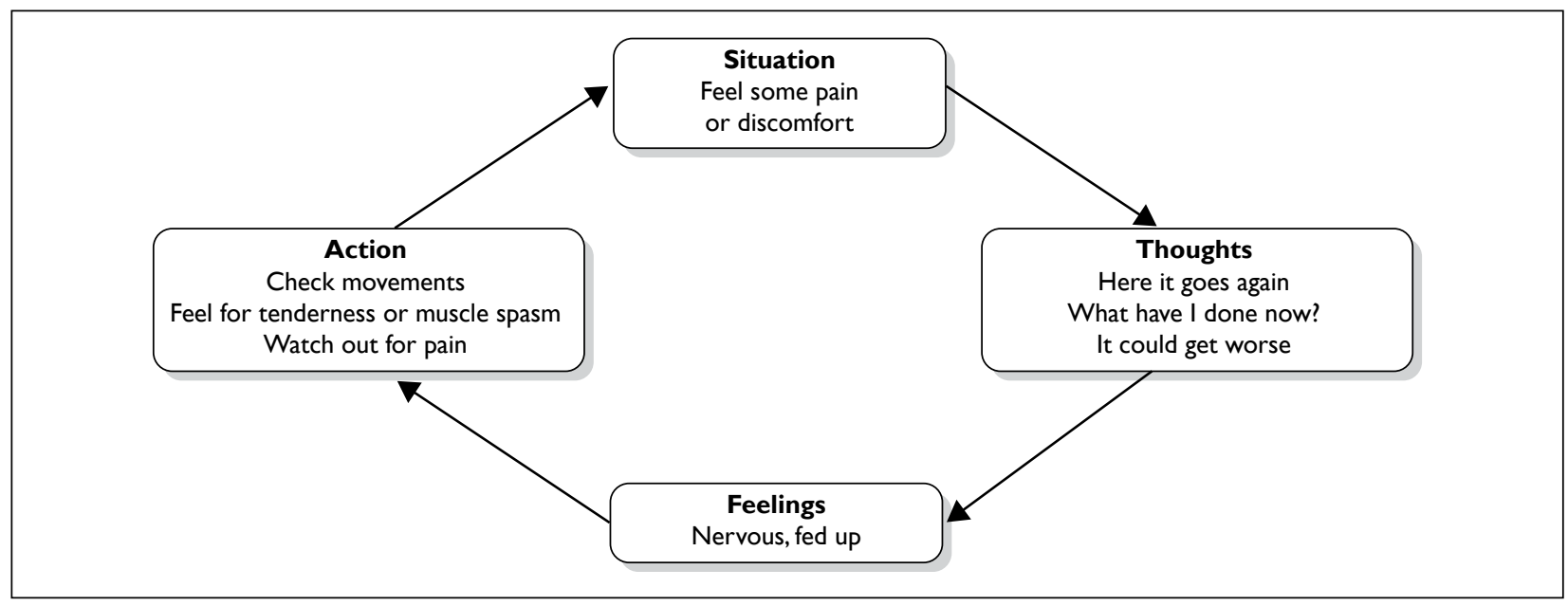

FIGURE 3 Hypervigilance cycle used in BeST session 5.

to cover the key components of $\mathrm{CBA}$ as defined by the BABCP. ${ }^{41}$

\section{Group size, equipment and space required for running the groups}

The only equipment needed was exercise mats, a flipchart easel, pad and pens. The optimal size for a therapy group has not been determined. A size of six to 10 is popular in practice as it is thought to encourage group discussion and problem-solving. Therefore we determined that the venue had to be sufficiently large to seat up to 10 participants in a circle with space to move around and place exercise mats on the floor during the break if participants wanted to do some exercises or have their exercises reviewed by the therapist running the group.
Before running the groups, each therapist was provided with all the necessary paperwork and materials.

\section{Targeting of specific risk factors}

Catastrophic thoughts were targeted in several ways: pain education, teaching participants how to identify these thoughts and challenging beliefs, specific education on fear avoidance and hypervigilance using vicious cycles, and through the therapist's use of questioning. The CB model identifies three levels of thinking. ${ }^{50}$ The most 'superficial' of these is negative automatic thoughts and these will tend to directly relate to the present situation. For example, a participant faced with a lifting task might have the thought 'I'll hurt myself'. Other levels of thinking include 
'assumptions' or 'rules' such as 'In order to be safe, I need to be careful at all times' and 'core beliefs' or 'schema' such as 'I am vulnerable'. These two deeper levels of thinking are usually related to more global domains about the person. They can be targeted directly within the context of CB psychotherapy. Within the trial, we trained health professionals to identify the negative automatic thoughts related to LBP. The questioning techniques that were adopted by the therapists in the trial were not designed to delve for deeper meanings such as the 'downward arrow technique', ${ }^{90}$ but to help patients explore alternative ways of thinking around their LBP, known as 'guided discovery'. ${ }^{91}$

Depression and underactivity were targeted through education on the effects of inactivity and the benefits of exercise, and through providing participants with the skills needed to increase their activity levels: goal setting, baseline setting, pacing and techniques to manage increases in pain.

Overactivity was targeted through thought challenging and developing pacing skills.

Passive coping was addressed through developing problem-solving skills and alternative helpful pain management techniques, such as relaxation, using medication and planning for potential flare-ups.

\section{Participant information folder}

A high-quality participant information folder was designed for the intervention and provided to each participant at the first assessment appointment
(Figure 4). The folder contained an overview of the sessions, how and where the groups would be run, therapist contact details, what participants should expect and exercise sheets. During the assessment these exercise sheets were personalised and the goal sheets were filled in. To support participants in working towards their goals while waiting for the group sessions to start, there were information sheets on goals and how to set baselines (starting level for activity). In addition, there were information sheets on sleep, medication and communicating with health professionals.

At each group session participants were given sheets to add to their folders that summarised the content of the session and provided details of their 'homework' task. If a participant missed any sessions, either the inserts were provided at subsequent sessions or they were sent through the post if the participant was unable to attend any other sessions. As the folder was only provided at the therapist assessment, any participant who did not attend for this session received only the active management intervention.

\section{Pilot study and refining of the intervention}

Several pilot groups were run as part of the study pilot procedures. An independent researcher collected feedback comments, which were given anonymously to the intervention development team. Comments were very positive, although more supervision of exercises was requested. As a result the therapists were instructed to ensure that each 
participant had at least one opportunity to have their exercises reviewed during the programme.

\section{Therapist recruitment and training}

A 2-day programme was developed to train registered health professionals (physiotherapists, psychologists, nurses or occupational therapists). The length of training reflects the typical length of informal training programmes for qualified staff currently available in the UK. We trained a spectrum of health professionals. This was a pragmatic decision - LBP is a common condition and there are unlikely to be sufficient numbers of psychologists to meet demand. It is recognised that generic pain management skills cross professional boundaries. ${ }^{92}$ For example, when CBAs have been applied in diabetes self-management, the treatment effect sizes are found to be the same for psychological specialists and non-specialist clinicians. ${ }^{93}$ Although there are high rates of distress and low mood among LBP sufferers in primary care, these symptoms are generally not severe. Therefore, a prolonged training programme in psychological management for the therapists in the trial was not felt necessary.

With this in mind the training was designed to ensure that individuals with different professional backgrounds would be equipped with the same basic knowledge and skills and that the intervention was effective regardless of which health-care professional delivered it. This included equipping psychologists with knowledge of LBP, physical activity and exercise prescription.

The training covered an understanding of LBP and the risk factors associated with chronicity, understanding the $\mathrm{CB}$ model, developing basic $\mathrm{CB}$ skills such as questioning techniques, developing group facilitation skills and learning the topics to be covered in each session, including pain management techniques. Training was delivered by a cotrained CBT therapist/physiotherapist and a clinical psychologist and was documented in an extensive training manual. ${ }^{49}$

\section{Therapist support}

In addition to the training already described, each therapist received a comprehensive treatment manual detailing the rationale and content of each session, including suggested dialogue. In response to feedback from the first training session a website and DVD were developed. The website contained all support materials, including trial updates and a 'frequently asked questions' section; a screen print is shown in Figure 5. The website also had a forum for posting questions to be answered by the trial intervention team and by other therapists. This section was not used; therapists preferred to e-mail or phone the trial team directly.

The DVD was a recording of sessions 1 and 3 run for volunteers from the research department as group participants. Feedback from therapists indicated that they found the DVD very useful before running the programme for the first time.

Within psychological therapies it is usual practice to have regular supervision sessions with a senior therapist to discuss cases. This is not normal practice within most of the other professions allied to medicine. For the purposes of the trial we allowed for flexible supervision, which consisted of discussions either face-to-face on-site visits or via phone or e-mail, whichever was convenient and appropriate for the issues to be discussed. Supervision was provided during the course of the trial by the clinical research fellow, which was on average 1.5 hours per group run. This supervision usually centred on difficulties encountered in the groups, for example difficulties in setting goals with some individuals.

\section{Satisfaction with training}

All therapists were asked to provide anonymous feedback in a questionnaire sent out 2 weeks after training; $72 \%$ of therapists rated the training as 'very good' with the remaining $28 \%$ rating it as 'good'. Confidence to deliver the intervention was rated as 'fairly confident' or 'very confident' by at least $75 \%$ of all therapists for each of the skills taught. The exception was 'thought challenging'. A significant proportion of therapists rated themselves as 'a little confident' (45\%) or 'not at all confident' $(10 \%)$ in these techniques. As this skill was new to the majority of therapists this rating was to be expected.

\section{Assessment of treatment fidelity}

Quality of the intervention delivery was checked via three processes:

- a site visit was made to a group session for each therapist during their first programme of intervention 


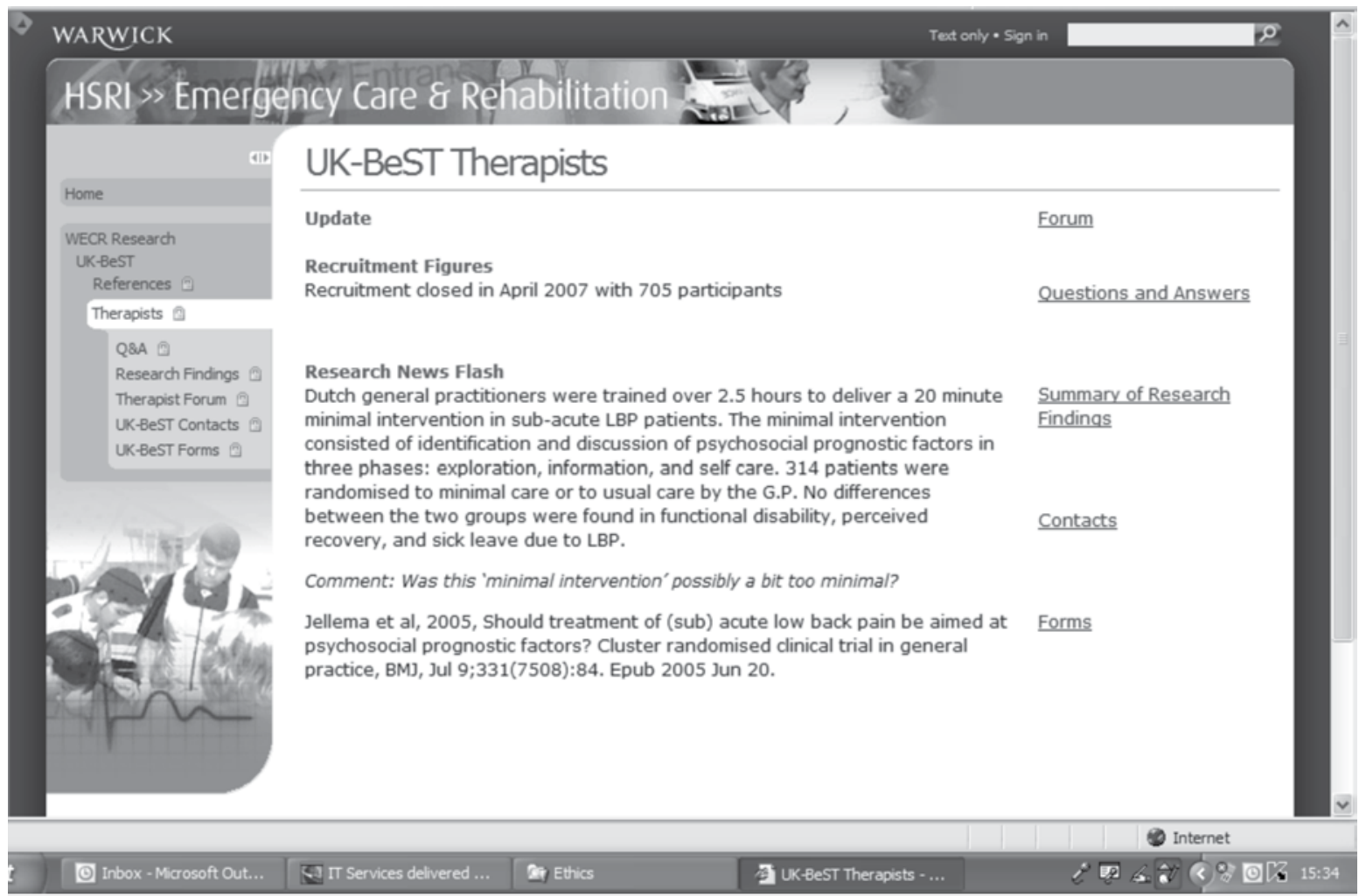

FIGURE 5 Screen print of BeST Therapist webpage.

TABLE 3 Session content and therapist skills

\begin{tabular}{|c|c|c|c|c|}
\hline Item & & $\begin{array}{l}\text { Not achieved } \\
\text { (\% of total) }\end{array}$ & $\begin{array}{l}\text { Partially achieved } \\
\text { (\% of total) }\end{array}$ & $\begin{array}{l}\text { Satisfactorily achieved } \\
\text { (\% of total) }\end{array}$ \\
\hline \multirow[t]{7}{*}{ Content } & Set agenda & $5(14)$ & $8(23)$ & $22(63)$ \\
\hline & Homework reviewed & $0(0)$ & I (3) & $34(97)$ \\
\hline & Topics covered & $0(0)$ & $3(9)$ & $32(91)$ \\
\hline & Break & $0(0)$ & $0(0)$ & $35(100)$ \\
\hline & Homework set & $0(0)$ & $5(14)$ & $30(86)$ \\
\hline & Feedback elicited & $6(17)$ & $7(20)$ & $22(63)$ \\
\hline & Exercises checked in break & $0(0)$ & I (3) & $34(97)$ \\
\hline \multirow[t]{8}{*}{ Style } & $\begin{array}{l}\text { Encouraged group } \\
\text { participation }\end{array}$ & $0(0)$ & $4(\mathrm{II})$ & 31 (89) \\
\hline & Listened appropriately & $0(0)$ & $0(0)$ & $35(100)$ \\
\hline & Empathy demonstrated & $2(6)$ & I (3) & $32(91)$ \\
\hline & Elicited beliefs/thoughts & $2(6)$ & II (3I) & $22(63)$ \\
\hline & $\begin{array}{l}\text { Questioning style } \\
\text { demonstrated }\end{array}$ & $2(6)$ & $8(23)$ & $25(7 \mathrm{I})$ \\
\hline & Referred to CB model & $4(\mathrm{II})$ & $4(\mathrm{II})$ & $27(77)$ \\
\hline & $\begin{array}{l}\text { Appropriate pacing of } \\
\text { session }\end{array}$ & I (3) & $7(20)$ & $27(77)$ \\
\hline & Appeared professional & $0(0)$ & $0(0)$ & $35(100)$ \\
\hline \multirow[t]{2}{*}{ Environment } & Comfortable & $0(0)$ & I (3) & $34(97)$ \\
\hline & Spacious & $0(0)$ & $0(0)$ & $35(100)$ \\
\hline
\end{tabular}


- $\quad$ subsequent groups had one session randomly selected for audio recording

- treatment records were screened for each participant.

The session content and skills demonstrated by the therapist were assessed via these processes using a checklist that included items shown in Table 3. The audio recordings were used as an observational tool as part of a process of evaluation to determine treatment fidelity, and were not used for further training and feedback.

\section{Summary}

A CB intervention was designed that targeted known modifiable risk factors for the development of chronic disabling LBP. The intervention adhered strongly to the principles of a CBA and was structured with sufficient flexibility to allow delivery in a variety of settings and by a range of different health professionals. The training and support package was practical to allow for roll out within the NHS if the intervention proved effective. 



\section{Chapter 3 \\ Methods}

\section{Aims}

There were two aims:

- To estimate the clinical effectiveness of AM in general practice versus AM in general practice plus a group-based, professionally-led CB package $(\mathrm{AM}+\mathrm{CBA})$ for subacute and chronic LBP in terms of:

- reduction in disability associated with LBP

- reduction of pain or improved tolerance of pain symptoms

- reduction of further medical, rehabilitation or surgical treatment for LBP

- improvements in quality of life.

- To measure the cost of each strategy, including treatment and subsequent health-care costs, over a period of 12 months and to estimate cost-effectiveness. The methods employed in the economic analysis are detailed in Chapter 6 .

In addition, we planned to interview a selection of participants to gain insight into the experience of LBP and of the treatments delivered as part of the trial (for the methods used, see Chapter 5).

\section{Research methods}

\section{Study design}

The study comprised a pragmatic, multicentred randomised controlled trial, with investigatorblinded assessment of outcomes.

\section{Setting}

Fifty-six general practices were recruited from seven regional clusters across the $\mathrm{UK}$.

\section{Practices}

The original plan had been to recruit clusters of general practices that had participated in the UK Back pain Exercise and Manipulation trial (UK BEAM) that had been run by the Medical Research Council (MRC) General Practice Research Framework (GPRF). ${ }^{94}$ However, when conducting the pilot study, it became evident that there were insufficient GPRF practices interested in participating in this new study within each area to ensure a flow of recruitment sufficient to sustain the group-based service delivery model. Therefore, alternative strategies were used. The majority of practices were recruited through collaboration with the primary-care trusts (PCTs), and had not been involved in the UK BEAM study. Fewer than $5 \%$ of practices were involved in the BEAM study. This strategy was implemented successfully, although it was labour intensive and caused a delay in starting recruitment. By June 2004, all PCTs in England had been contacted and where interest was expressed a series of visits was undertaken. Initially, seven PCTs were recruited. By the end of November 2004, two PCTs withdrew their consent to participate for logistical reasons, and by July 2005, five clusters (Coventry, Norwich, Langbaurgh, Heart of Birmingham and Solihull) had launched the BeST trial in their PCT. To speed up recruitment and meet target dates for the end of recruitment, two additional PCTs, South and North Warwickshire, were launched in January and March 2006 respectively.

\section{Participants}

Our aim was to recruit people with subacute and chronic LBP, who were experiencing symptoms that were at least moderately troublesome. Low back pain presents in a wide variety of ways, and typically patients present with a spectrum of severity and chronicity. The treatment for acute LBP (defined as a first episode that has occurred for less than 6 weeks) is agreed, and although not evidence based, it is generally recognised that psychological therapies are most likely to benefit those with subacute and chronic conditions. ${ }^{95}$ We wished to exclude people who had transient, minimally troublesome symptoms.

\section{Eligibility criteria}

The eligibility criteria were as follows.

\section{Inclusion criteria}

For entry into the trial, the following inclusion criteria had to be fulfilled: 
- $\quad$ participants had attended general practice reporting $\mathrm{LBP}$ of at least moderate troublesomeness for $>6$ weeks

- participants had to be aged 18 years or older

- participants were able to give informed consent.

\section{Exclusion criteria}

Participants were not eligible for BeST if they:

- $\quad$ were aged $<18$ years

- had been managed previously in a CB programme

- had factors associated with serious pathology [these included cauda equina symptoms, systemic illness (including cancer, human immunodeficiency virus infection, fever), widespread neurology, severe unremitting night-time pain, violent trauma (fall from height, road traffic accident) and substantial unexplained weight loss]

- had severe psychiatric or personality disorders sufficient to merit exclusion as determined by the GP.

\section{Treatments}

\section{Active management (reference group)}

Both the treatment and reference (control) groups received an intervention that was consistent with best practice in primary care (see Chapter 1 , Current management in the UK). Nurses were trained in the approach using a 1-hour training session that included methods to cascade the information within their practice environments. The nurses provided the AM to all trial participants and, in addition, provided each with a copy of The Back Book. ${ }^{35,96}$ The active management intervention lasted approximately 15 minutes.

\section{Cognitive behavioural therapy (for the experimental arm only)}

In addition to $\mathrm{AM}$, participants randomised to CBA received up to six sessions of group therapy (for details, see Chapter 2).

\section{Study procedures}

\section{From initial identification of participants to randomisation}

Participants consulting their GP in the previous 6 months with LBP were identified from computerised searches of primary-care records or from general practice attendances.

A list of potential participants was screened by GPs, who identified those patients who should be excluded on the basis of serious illness or mentalhealth problems. The GPs were encouraged to make prospective referrals of any participants they considered suitable for the trial, although only a small number of participants were referred this way. In some practices, searches were repeated after 12 months to identify further participants.

Once potential participants had been identified, an invitation letter (including an information sheet) to participate in the trial, and an initial approach questionnaire (see Appendix 1) was sent out to determine interest and eligibility. The GP signed the approach letter. Potential participants were given approximately 2 weeks to send the initial approach questionnaire back. If there was no reply after 2 weeks a second letter was sent out. Those participants who expressed an interest in the trial and appeared to meet the initial inclusion criteria were contacted by a research nurse and the first of two assessment appointments with the research nurse was scheduled. The reasons for decline/ withdrawal/exclusion were documented at all stages.

At the first nurse assessment, the trial was explained in more detail to the participant. In general these appointments were carried out face-to-face at the participant's own GP surgery, although sometimes the appointment was conducted over the telephone. Each of the nurses was provided with a laptop and the nurse determined whether the subject was eligible by completing the computerised first nurse assessment questionnaire (see Appendix 2). Those participants who were temporarily excluded (e.g. because they were awaiting or receiving treatment) were issued a temporary exclusion letter (see Appendix $3)$. The temporarily excluded participants were allowed to enter the trial once they had become eligible by contacting the nurse and arranging an appointment. For those participants interested in taking part in the trial who met all the study inclusion criteria, a second appointment was made (appointment two - nurse randomisation appointment). This appointment was arranged for within 1-2 weeks of the first nurse appointment to allow time for the participant to consider whether they would like to enter the trial. For those participants who did not attend either of the two 
nurse appointments, a reminder telephone call was made and the opportunity was provided for another appointment to be made if the participant wished.

At the nurse randomisation appointment, the nurse randomisation assessment questionnaire (see Appendix 4) was completed. If the participants satisfied the eligibility criteria and provided informed consent then they were asked to (1) sign the informed consent form (see Appendix 5) and (2) complete the baseline questionnaire (see appendix 6). Every participant randomised then had an active management session supplemented by The Back Book ${ }^{96}$ and it was stressed that they should read and follow the advice given.

The nurse then filled in the randomisation form (see Appendix 7). The information on this checklist was telephoned through to the randomisation office at the MRC Clinical Trials Unit in London (see Randomisation, below). The unit then provided the nurse with the additional treatment allocation for that participant. The nurse would then let the participant know to which treatment they had been randomised. If the randomisation appointment occurred at a time when the randomisation office was closed, the randomisation form was faxed and the treatment allocation was provided once the office reopened.

\section{From randomisation to follow- up}

For those participants randomised to $\mathrm{AM}+\mathrm{CBA}$, the nurse sent a notification letter to the therapist and provided the participant with a copy. The nurse also advised the participant that the therapist would contact them to arrange a time to start their treatment within the next 2 weeks and asked the participant to contact the therapist if this did not happen. The nurse e-mailed the therapist with confirmation of final numbers referred into the group to check that the number of referrals made matched the number of referrals received.

\section{Follow-up}

Follow-up was conducted at 3, 6 and 12 months after randomisation using self-administered questionnaires. The majority of questionnaires were completed in postal format (see Appendix $8)$. Response was tracked carefully by the trial office, and a reminder questionnaire was sent after 2 weeks if a follow-up questionnaire had not been returned. If the questionnaire had still not been returned after a further 2 weeks, participants were telephoned to check that the questionnaires had been received and to arrange for another to be sent if needed. If the questionnaire remained unreturned after a further 2 weeks a telephone call was made to the participant to request a core set of data that was collected over the telephone at a time convenient to the participant. All people who provided core outcomes were willing to continue to participate in the trial. Core outcomes are detailed in Appendix 9.

The clinical record form was stamped with the date and initialled on receipt at the Warwick Trials Unit Office. It was then checked for correctness and completeness and coded for the data input clerk. Any queries were checked with the statistician. Missing data were clarified with participants where possible.

The baseline and follow-up data were singleentered into the database by the data input clerk. The accuracy of the data entry was checked by taking a $10 \%$ random sample (70 participants) and completing a $100 \%$ correctness check on all the variables on the database against the record forms (baseline, 3, 6 and 12 months). There is no firm guidance as to what constitutes an acceptable error rate. Shen ${ }^{97}$ suggests a very conservative error rate of $0 \%$ for the primary outcome and $0.5 \%$ for the secondary outcomes. This was achieved in BeST.

The baseline and follow-up data were validated continuously through the trial and reported at intervals to the Data Monitoring Committee (see Data monitoring and ethics committee, p. 27). After the final validation checks, the database was 'frozen'. If changes were required thereafter, these were formally documented. The clinical outcome data were validated in SAS (SAS Institute, Cary, NC, USA). The health economic data were validated in SAS and then transferred to STATA (Statacorp, College Station, TX, USA) for analysis.

The validation checks carried out included:

- $\quad$ eligibility criteria

- consecutive date checks

- range checks

- missing data checks. 


\section{Outcome assessments}

\section{Demography and baseline assessments}

Appendix 11 lists the demographic and clinical data that were collected at each of the three prerandomisation stages. The baseline assessments are listed in Appendix 6.

The data included date of birth, sex, LBP/ symptoms in the past 6 weeks, frequency of back pain in the past 6 weeks, troublesomeness in the past 6 weeks, ethnic origin, age when left full-time education and employment details.

Although we had proposed to collect the Acute Low Back Pain Screening Questionnaire (ALBPSQ) ${ }^{98}$ as part of the baseline assessment, this was dropped in the set-up phase of the study to reduce respondent burden, and in recognition that many of the other measures were capturing duplicate information.

\section{Clinical outcomes}

We were guided in the selection of outcome measures by three principles. First, we wished to be consistent with the international recommendations for clinical trials of LBP interventions. ${ }^{99,100}$ Second, we considered more recent methodological studies of LBP outcome measures. Third, we did not want to overburden participants with too many outcomes.

The International Forum of LBP has recommended that trials should measure five domains: pain symptoms, function, generic health status, work disability and satisfaction of care. ${ }^{99,100}$ Table 4 details the measures used in this trial ${ }^{101}$ and the time points of data capture.

A copy of all follow-up questionnaires is provided in Appendix 8.

\section{Primary outcomes}

We selected two primary outcome measures a priori because of concerns with the scaling of the Roland Morris Questionnaire.

\section{Roland Morris Questionnaire}

The Roland Morris Questionnaire (RMQ) is the most widely used measure of LBP disability in primary-care trials. Originally derived from the Sickness Impact Profile, it contains 24 items relating to a range of functions commonly affected by LBP. ${ }^{102}$ It takes less than 5 minutes to complete. It has good reliability ${ }^{101}$ but there are concerns that it does not conform to many of the assumptions that underpin its use in statistical analysis (scaling

TABLE 4 Outcome measures used in the Back Skills Training Trial

\begin{tabular}{|c|c|c|}
\hline Domain & Measures & Time points \\
\hline \multicolumn{3}{|l|}{ Primary measures } \\
\hline Pain-associated disability & Roland Morris Questionnaire ${ }^{102}$ & $0,3,6,12$ \\
\hline Pain & Modified Von Korff Scale ${ }^{103}$ & $0,3,6,12$, Tel $^{\mathrm{a}}$ \\
\hline \multicolumn{3}{|l|}{ Disability } \\
\hline \multicolumn{3}{|l|}{ Secondary measures } \\
\hline Occupational and other limitations & Numbers of days off work, reduced activity and bed rest & $0,3,6,12$, Tel $^{\mathrm{a}}$ \\
\hline Health-related quality of life & Short Form- 12 version $2^{104}$ & $0,3,6,12$, Tel $^{\mathrm{a}}$ \\
\hline Back pain beliefs & ${ }^{a}$ Fear avoidance scale (first five items only) ${ }^{105}$ & $0,3,6,12$ \\
\hline Self-efficacy & Pain self-efficacy questionnaire ${ }^{106}$ & $0,3,6,12$ \\
\hline Satisfaction with treatment & Single-item rating of satisfaction with treatment ${ }^{99}$ & $3,6,12$ \\
\hline Global rating of change & Seven-point rating ${ }^{99}$ & $3,6,12$ \\
\hline \multicolumn{3}{|l|}{ Economic analysis } \\
\hline Resource use & Resource use questionnaire & $3,6,12 \mathrm{Tel}^{\mathrm{a}}$ \\
\hline $\begin{array}{l}\text { Health-related quality of life; time } \\
\text { trade-off score }\end{array}$ & EuroQoL five dimensions (health utility) ${ }^{107}$ & $0,3,6,12, \mathrm{Tel}^{\mathrm{a}}$ \\
\hline
\end{tabular}


and normality of distribution). Data from the Oxfordshire Low Back Pain Trial suggested that it had a marked ceiling effect, failing to capture important clinical information on improvement in participants with subacute or chronic LBP attending NHS physiotherapy. It has been shown to be differentially sensitive at low, mid and high ranges, with (not unsurprisingly) better sensitivity in the middle range. ${ }^{108,109}$ In the low to mid range, the RMQ is less sensitive to within-group changes than the Aberdeen Low Back Pain Score, but better at detecting between-group differences. ${ }^{101}$

\section{Scoring}

Completion of the RMQ scale comprises a mark next to each appropriate statement.

\section{Interpretation}

The total number of marked statements is added up to form a score (out of 24), and a low score is associated with less disability.

\section{Modified Von Korff Scale}

The Modified Von Korff Scale (MVK) ${ }^{103}$ assesses two dimensions - pain and disability associated with back pain in the last 4 weeks. It is made up of six items, each of which is scored on a scale of 0 (no pain/disability) to 10 (worst pain/disability). The first three of these items relate to disability and ask about how back pain interferes with (1) daily activity, (2) recreation and (3) ability to work. The last questions relate to pain and assess the (1) worst pain, (2) average pain and (3) rating of back pain today. The questionnaire was administered at baseline, 3, 6 and 12 months.

\section{Scoring}

The scale has two dimensions:

$\mathrm{MVK}_{\text {disability }}:$ Disability score $=[$ (sum points of the first three questions)/3] $\times 10$

$\mathrm{MVK}_{\text {pain }}:$ Pain score $=[($ sum points of the last three questions) $/ 3] \times 10$.

\section{Interpretation}

The higher the score, the more severe the disability or back pain.

\section{Secondary outcomes Occupational disability and limited activity days}

Three separate questions were used to elicit the number of days in the period from 0 to 3 months, 3 to 6 months and 6 to 12 months that participants:
- had to cut down on normal activities (for more than half a day)

- had time off work because of low back or leg pain (sciatica).

These questions were recommended by the International Forum, and have been used widely (summarised in Deyo et al. ${ }^{99}$ ).

\section{Participant satisfaction}

Participant satisfaction was assessed using the single-item question recommended by the International Low Back Pain Forum: 99 'Over the course of treatment for your LBP or leg pain, how satisfied were you with your overall medical care?' We modified this question slightly to reflect care over the duration of the study, and by changing the term 'medical care' to 'one related to health care'. The question that was asked was 'How satisfied are you with the treatment you received?' Responses were rated on a five-point Likert scale, ranging from 'very dissatisfied' to 'very satisfied'.

\section{Psychological and behavioural measures}

We included these measures because they measure constructs hypothesised to lie on the causal pathway of effect, and we hoped that they would provide some explanation as to why the treatment may or may not be effective.

\section{Fear avoidance beliefs questionnaire}

The Fear Avoidance Beliefs Questionnaire (FABQ) is a measure of the degree of fear of pain and disability, and the avoidance of physical activities that can result. Each item is scored from 0 (completely disagree) to 6 (completely agree). This scale has two dimensions (fear avoidance beliefs about work and fear avoidance beliefs about physical activity). We selected only the section of the measure concerned with physical activity as it has generic applicability.

Scoring The scores from each of these items are summed to provide a total score. Minimal scale score is 0 and maximum scale score is 24 .

Interpretation The higher the scale scores the greater the degree of fear and avoidance beliefs shown by the participant.

\section{Pain self-efficacy}

This is a measure of the patient's confidence to carry out a range of activities despite the back pain. There are 10 items, ranging from 0 (not at all confident) to 6 (completely confident). 
Scoring The scores are totalled with the total ranging from 0 to 60 .

Interpretation A lower score indicates reduced selfefficacy of the participant.

\section{Quality of life}

Quality of life was measured using the Short Form12 (SF-12) version 2. The SF-12 (a short version of the SF-36) is a measure of health-related quality of life and is widely used in back pain trials. The SF12 has performed well in previous clinical trials of LBP using postal follow-up. ${ }^{110}$

\section{Scoring}

The SF-12 manual was used to score the SF-12. Results were expressed in terms of two meta-scores: physical and mental components.

\section{Interpretation}

The SF-12 is scored so that a high score indicates better physical functioning. The physical and mental scores have a range of 0-100 and were designed to have a mean score of 50 and a standard deviation (SD) of 10 in a representative sample of the US population (see Table 5 for age-specific SF-12 scores). Scores $>50$ therefore represent above-average health status. On the other hand, people with a score of 40 function at a level lower than $84 \%$ of the population (one SD) and people with a score $<30$ function at a level lower than approximately $98 \%$ of the population (two SDs).

\section{Health economics - EuroQoL five dimensions}

The EuroQoL five dimensions (EQ-5D) $)^{111}$ measure was collected at baseline, 3, 6 and 12 months' follow-up.

The instrument (see Appendix 8) contains a description of the health state in five dimensions or items: mobility, self-care, usual activities, pain/ discomfort and anxiety/depression. It measures health on five dimensions and a tariff is available for deriving a single utility score. Completion takes less than 5 minutes. ${ }^{107}$

\section{Scoring}

The items are three levels of severity for each item: 1 (no problems), 2 (some problems) and 3 (unable to do/extreme problems). For each item, the respondents must indicate the level of severity that best describes their personal health state at the time of giving the answers. The subject's global health state is defined as the combination of the level of problems for each of the five dimensions. Health states defined by the EQ-5D can be converted to a single summary by applying scores from a standard set of weights (or preferences) derived from general population samples. ${ }^{107}$

\section{Interpretation}

The weightings represent the strength of societal preference for a described health state and are scored between 0 (death or worst imaginable health state) and 1 (full health or best imaginable health state). The quality adjustment is then multiplied by the expected life-years in the assessed health state to arrive at the total quality-adjusted life-years (QALYs) achieved. The total utility associated with a health-care intervention is then the sum of utility weights declared by respondents multiplied by the time spent in those states.

\section{Resource use questionnaire}

Resource use was monitored for the economic analysis and to gain insight into other treatments being used either as a consequence of or alongside the treatments being tested in the trial. A selfreport questionnaire was administered to ascertain whether participants had additional hospital treatment for their LBP during the follow-up period, specifying whether this was NHS or private treatment; any GP consultations; and any manipulation, massage, etc. which they received during follow-up. Participants were also asked about the number and types of any medications and treatments, including pain-relieving medications. Participants were asked to distinguish

TABLE 5 Short Form-12 mean scores (general US population)

\begin{tabular}{lll} 
Age (years) & Physical component & Mental component \\
\hline $45-54$ & 50 & 50 \\
$55-64$ & 47 & 51 \\
$65-74$ & 44 & 52 \\
$\geq 75$ & 39 & 50
\end{tabular}


between prescription and out-of-pocket expenses. We used a structured closed questionnaire to ascertain these data, based on a questionnaire that had been used in previous LBP trials. ${ }^{37}$ Participant self-reported information on service use had been shown to be accurate in terms of intensity of use of different services. ${ }^{112}$

\section{Other treatments}

There was a possibility that participants would seek other forms of treatment during the follow-up period. The resource use questionnaire allowed us to monitor changes in the amount or types of analgesia used, use of physical treatments (osteopathy, chiropractic or physiotherapy), alternative therapies, or referral to secondary-care services. Participants and GPs were encouraged to refrain from referral of participants to other treatments where possible during the first 3 months after randomisation and while participants were attending the CBA course.

\section{Randomisation}

\section{The randomisation system}

Initially a web-based randomisation system was planned. This system was very difficult to implement as it depended on the research nurses being able to access an NHS web link or secure internet link in the practices. The nurses were not always able to have access to practice computers and were often allocated rooms without internet access. Instead, an independently administered telephone randomisation service was used at the MRC Clinical Trials Unit in London. Random allocations were generated by an independent statistician in a ratio of $2: 1$ in favour of the intervention arm of the trial. Randomisation reports were sent to the trial office on a weekly basis.

\section{Method of randomisation}

We used stratified block randomisation. The randomisation was stratified by region (recognising the heterogeneity likely to exist between regions). It was important to balance the severity of back pain (moderately versus very/extremely troublesome) over the two treatment arms in case there was a difference in response based on severity. Block lengths were sufficiently large to ensure that predictions of treatment allocation could not be made, and that the allocation ratio of $2: 1$ could be achieved.

In the initial application, randomisation was based on equal assignment (a ratio of $1: 1$ ) over the two therapy groups. It was recognised, during the trial set-up stages, that such an allocation would produce an insufficient yield of participants to run the groups for the AM+CBA arm. As a result, random allocation was conducted using a $2: 1$ $(\mathrm{AM}+\mathrm{CBA}: \mathrm{AM})$ ratio. This had little impact on the power of the trial and considerably improved efficiency.

The details required by the randomisation officer are shown on the randomisation form (see Appendix 7).

\section{Quality assurance}

We implemented a quality assurance protocol to monitor and ensure that research nurses were adherent to the trial protocol. Each research nurse was visited on at least two occasions by a senior research nurse from the MRC GPRF, and observed taking consent and delivering the active intervention component of the intervention. Problems were minimal.

\section{Compliance}

We measured compliance with the intervention by the number of sessions attended. This information was ascertained from records collected by the therapist providing the treatment.

\section{Formal approvals}

The original ethics approval for the project was dated 26 March 2003 with a substantial amendment approving protocol amendments on 22 July 2004. Further amendments for the qualitative interview study and consent, change to patient information sheets and promotional posters were all approved on 5 March 2005. An amendment to the randomisation procedure was submitted on 24 May 2005 and approved via chairman's action on 14 July 2005. Further chairman's action approval was received on 7 September 2005 for a change in trial personnel. A substantial amendment was approved on 25 January 2006 for version control. Chairman's action was sought on 5 July regarding 
follow-up procedures and approval was received on 1 September 2006.

\section{Adverse events}

\section{Risks and benefits}

The risks to participants in this trial were considered small. The potential benefits were minimisation of back pain symptoms and prevention of chronic problems.

\section{Potential side effects and monitoring}

It was anticipated that there were unlikely to be any serious side effects from the treatments. Possible potential side effects were worsening of symptoms if other more effective treatments were withheld. All adverse events were reported to the Data Monitoring and Ethics Committee (DMEC). Serious adverse events were defined as those that resulted in death or admission to hospital as a result of the intervention, or that caused unwarranted distress to a participant. All deaths and potential events were reported to the Chief Investigator, who determined whether the event might have been or was attributable to the intervention. Events were reported to the ethics committee and to the DMEC.

\section{Sample size}

The primary outcome measures were the RMQ and MVK assessed over 12 months.

Increasingly it has been recognised that advances in modern health care are most likely to yield moderate improvements, but in the context of highly prevalent conditions like LBP, these are considered worthwhile. ${ }^{113}$

\section{Choice of treatment effect}

Deciding the minimal clinically important difference (MCID) between groups was problematic, particularly for the RMQ. Previous trials (including the UK BEAM, Oxfordshire Low Back Pain Trial and York Back Pain and Exercise Trial) adopted a clinically significant difference between groups of 2.5 RMQ points, based on the views of an expert group of clinicians and researchers. This equates to a large standardised effect size of $0.65,{ }^{114}$ assuming an SD of 4.0. observed in several large trials (effect sizes were 0.35 for BEAM and 0.36 for the York Low Back Pain Trial ${ }^{155}$ ). Careful back tracking through trials (reviewed by Bombardier et al. ${ }^{100}$ ) suggested that the MCID had been derived from a few studies of short-term benefits ( $<8$ weeks) of therapies in LBP. This is the stage at which one would expect to see the largest differences between groups because of the natural history of LBP. Powering a trial on the short-term clinical benefit was unlikely to be sufficient to monitor longer-term impacts of public-health significance. The majority of outcomes reported for CBA suggest moderate benefits at 1 year, with a between-group effect size of approximately 0.35 for the majority of outcomes reported in efficacy trials. ${ }^{116}$ This equates to a between-group difference of approximately 1.4 change points on the RMQ disability score (i.e. new treatment approaches are approximately half as good again as the comparative treatment at reducing disability). We therefore considered that an effect size of 0.35 would be a suitable target for the CBA to be worthwhile.

\section{The power of the trial}

We selected a power of $90 \%$ recognising that economic analyses required greater power. Based on the experience of previous trials, the number of participants we intended to recruit was adequate for the purposes of the economic analysis. ${ }^{17}$

We selected a significance level of 0.01 because of the need for a definitive trial. The sample size was sufficient to detect worthwhile benefits in the range of secondary outcome measures at conventional levels of statistical significance.

\section{Adjustment for cluster effects of group interventions}

The unit of randomisation was the individual, but the sample size estimate was inflated to account for the occurrence of cluster effects relating to grouping of patients together in each $\mathrm{CB}$ programme, and the clustering of outcomes around individual therapists (therapist effects). Previous studies of LBP report the intracluster correlation coefficient (ICC) for therapist effects to be $0.01,{ }^{94}$ although comparative data on group effects were not available. The ICC of 0.01 was used as an estimate for the therapist and group effects. Based on collecting outcome data on an average of seven people per group we inflated our sample size by 1.07. 


\section{Loss to follow-up}

We assumed a loss to follow-up of $25 \%$ at 12 months, as achieved in the BEAM trial. ${ }^{94}$

\section{Sample size estimate}

The sample size was estimated at the outset of the trial, and subsequently revised during trial set-up as we examined the practical aspects of setting the trial up. In the original sample size estimate [Health Technology Assessment (HTA) application], we used an effect size of $0.35 \mathrm{SD}$ points, $90 \%$ power and $p<0.01$ and inflation factor for clustering yielding an estimate of 262 in each group. We specified that we wished to detect a between-group difference of approximately 1.4 change points and a standardised effect size of 0.35 . Assuming loss to follow-up of $25 \%$ and a balanced allocation between treatment and control $(1: 1)$, we aimed to recruit 350 in each group (700 in total).

As we progressed with the early phases of the trial, and with the experience gained in another trial we were conducting of group treatments, we opted to use an unbalanced randomisation (2:1 in favour of the intervention). The reason was pragmatic; we could not sustain a flow of participants to fill the groups in a reasonable time frame using balanced randomisation. A randomisation balance of $2: 1$ can be adopted with inconsequential loss of power, but further imbalances necessitate an increase in study size. ${ }^{118,119}$ We estimated that a change to unbalanced randomisation without further inflation of the sample size would still allow us to detect clinically worthwhile benefits (effect size 0.42 and a between-group difference of $1.8 \mathrm{RMQ}$ points. ${ }^{118}$ We chose not to increase the sample size target, recognising that if loss to follow-up was $25 \%$ as predicted the study still had power to detect clinical worthwhile improvements. At the end of the trial, follow-up was better than anticipated, and we retained power to detect the original differences specified.

Using the proposed 700 participants and a $2: 1$ randomisation, we needed approximately 233 participants in the AM group and 467 participants in the $\mathrm{AM}+\mathrm{CBA}$ arm.

\section{Pilot study}

We undertook a series of pilot studies to refine the study procedures and ensure that the intervention was acceptable and deliverable in the format intended.

The intervention was piloted at the Nuffield Orthopaedic Centre NHS Trust in Oxford in the first instance. Two CBA cycles were completed and participants provided feedback on the contents and revised contents of the intervention. We then piloted the intervention in primary care along with the study procedures.

We planned to carry out the pilot study in three general practices in the Coventry area and to recruit 30-40 participants. In fact it was carried out in only one of the practices for the following reasons:

- one practice dropped out when they learnt more about the trial

- we were forced to run the pilot over the July/ August period because of a 7-week delay in the Central Office for Research Ethics Committees confirming ethical approval because of an internal Central Office for Research Ethics Committees communication problem (as a consequence the research nurse who was secured to run the second practice was unavailable - she had to cover leave, went on holiday and was subsequently stranded abroad by hurricanes; however, this practice participated in the main trial).

We tested one of the two recruitment strategies identification of participants through participant record searches but not prospective identification of participants via the GPs. In general the pilot study had been successful and useful in refining the study methods and in particular, methods related to the approach to participants.

\section{Data management}

All the databases were developed in Microsoft ACCESS 2002.

The prerandomisation and randomisation information was captured on laptops, whereas the baseline and follow-up data were collected using postal clinical research forms or by telephone and then entered into the database manually. Computerised validation checks were incorporated into the data sets to minimise data errors. 
Database specifications were set up by the statistician and programmer for each variable collected at baseline and follow-up assessments.

\section{Statistical analysis \\ Prerandomisation and randomisation}

The trial has been reported in accordance with the CONSORT ${ }^{120}$ guidelines that have now been extended to consider the reporting of complex interventions. All statistical tests were two-sided. The statistical analyses were carried out using SAS (version 9.13) and STATA (version 9). The health economic analyses were carried out using STATA. The demographic profile of the sample was summarised as the mean, SD, range and number of participants missing. The categorical data have been summarised using the number (and percentage) of participants within each category.

\section{CONSORT flowchart}

The CONSORT flowchart details the number (and percentage) of participants who were recruited into the trial from the second nurse's assessment time point. The flowchart depicts the passage of participants through the trial (prerandomisation, intervention allocation, follow-up and primary data analysis).

\section{Recruitment of randomised participants}

The number (and percentage) of participants at baseline and follow-up was detailed as follows:

- with data/clinical research forms present

- with core data present

- with no data present.

The follow-up (response) rates were derived from the total data present (i.e. sum of first two bullet points). The cumulative number of withdrawals over each time period was calculated and the frequency of loss to follow-up, blank questionnaire return and withdrawal (with reasons) was summarised.

The primary analysis method was 'intention-totreat'. The participants were analysed according to the therapy to which they were randomised, irrespective of the treatment they actually received.

Intention-to-treat formed the basis for computing the proportion of participants at different stages of the trial from randomisation to 12-month follow- up. The main summary tables and analysis are based on the intention-to-treat population unless otherwise specified.

\section{Per protocol analysis}

Participants who did not adhere to the treatment at the prespecified level of three or more sessions or who were incorrectly randomised were removed to form a per protocol sample. These analyses were undertaken using the primary outcomes and informed the sensitivity analysis.

\section{Clinical outcomes: baseline and follow-up}

Two sets of analyses were carried out:

- primary analysis observed case analysis

- sensitivity analysis missing data imputed at case level and questionnaire level and per protocol analysis.

\section{Change from baseline to each of the follow-up assessments}

Outcomes were summarised as the change from baseline score. The absolute scores were not used because the distribution of the data was, for some outcome measures, substantially non-normal. This was expected, particularly for the RMQ. A range of transformation methods were investigated, but none was able to normalise the data.

We needed to identify a method of summarising the data that would allow us to implement parametric methods including random effects and hierarchical modelling. Non-parametric covariance analysis ${ }^{121}$ is substantially limited in both scope and interpretation and was rejected because it does not allow consideration of clustering effects within the model. Similarly, more sophisticated statistical methods of modelling the probability density function ${ }^{122}$ while allowing for non-normality are significantly limited in interpretation and do not allow for consideration of clustering effects.

The interpretation of change from baseline scores is listed in Table 6.

Change from baseline is interpreted as 'improvement'/'deterioration' in condition from the point the participant provides the first (baseline) clinical assessments and this was found to be normally distributed for all the outcomes. This provided the only robust method of transforming the absolute scores. Although commonly reported 
in many trials, analyses based on change from baseline have to be interpreted with caution. Difficulties arise when there are differences in the central tendency and/or distribution of baseline scores between the randomised groups; ${ }^{123}$ this is not the case for BeST.

We investigated the time frame of improvements. We focused our interest on the average improvement over all follow-up time points, and on the benefit sustained at 12 months, as both indicators are of public health interest.

\section{Summary statistics (observed data) at each follow-up time point}

The absolute continuous questionnaire scores were summarised using means, SDs, median, range and the number of participants with and without the questionnaire score.

The effect sizes were calculated as the mean difference of the absolute values of the treatment effects at the relevant follow-up point divided by the pooled SD of baseline means.

\section{Statistical analysis - linear regression models (observed data) at each follow- up time}

Linear regression models were fitted to the data using the change from baseline values as the response. Unadjusted and adjusted (for baseline, age and sex) estimates (and standard errors) were obtained for the treatment effect with a $95 \%$ confidence interval (CI). Values of $p$ were obtained using the Wald method.

\section{Statistical analysis - random effect models (observed data) Incorporation of therapist/group session random effects}

Random effect regression models were fitted to estimate any potential group or therapist effects. The intervention is delivered as an assessment plus six group sessions. The intention was that once a cohort of participants was scheduled to a group, they would attend all of the sessions with that cohort, as opposed to changing between cohorts. The clinical rationale is that people build up a relationship with other participants over time. An additional potential effect arises from therapists (MRC Population Health Science Research Network workshop: Clustering effects in individually randomised controlled trials and other studies, 2007). We used hierarchical random effect models implemented in SAS (PROC MIXED) to model these effects.

Other effects, such as regional clusters and general practices were not incorporated into the models in a formal manner. Randomisation was stratified by regional clusters, and hence we do not anticipate any imbalance.

\section{Intracluster correlation coefficients}

The ICCs were used to quantify the clustering effects arising from therapist and group effects in the primary outcomes. The smaller the ICC, the less the clustering effect.

TABLE 6 Interpretation of the absolute and change scores

\begin{tabular}{|c|c|c|}
\hline $\begin{array}{l}\text { Questionnairel } \\
\text { score }\end{array}$ & Interpretation of absolute score & $\begin{array}{l}\text { Change from baseline (baseline - follow- } \\
\text { up) }\end{array}$ \\
\hline RMQ & $\begin{array}{l}\text { Lower score implies less disability; higher score } \\
\text { implies more disability }\end{array}$ & $\begin{array}{l}\text {-ve implies deterioration; +ve implies } \\
\text { improvement }\end{array}$ \\
\hline MVK (disability) & $\begin{array}{l}\text { Lower score implies less disability; higher score } \\
\text { implies more disability }\end{array}$ & $\begin{array}{l}\text {-ve implies deterioration; +ve implies } \\
\text { improvement }\end{array}$ \\
\hline MVK (pain) & $\begin{array}{l}\text { Lower score implies less pain; higher score } \\
\text { implies more pain }\end{array}$ & $\begin{array}{l}\text {-ve implies deterioration; +ve implies } \\
\text { improvement }\end{array}$ \\
\hline $\mathrm{FABQ}$ & $\begin{array}{l}\text { Lower score implies less fear/avoidance; higher } \\
\text { score implies more fear/avoidance }\end{array}$ & $\begin{array}{l}\text {-ve implies deterioration; +ve implies } \\
\text { improvement }\end{array}$ \\
\hline Pain self-efficacy & $\begin{array}{l}\text { Lower score implies less confidence; higher score } \\
\text { implies greater confidence }\end{array}$ & $\begin{array}{l}\text {-ve implies became more confident; +ve implies } \\
\text { became less confident }\end{array}$ \\
\hline $\begin{array}{l}\text { SF-I2 (physical and } \\
\text { mental) }\end{array}$ & $\begin{array}{l}\text { Lower score implies poor functioning; higher } \\
\text { score implies better functioning }\end{array}$ & $\begin{array}{l}\text {-ve implies functioning has improved; +ve implies } \\
\text { functioning has deteriorated }\end{array}$ \\
\hline
\end{tabular}




\section{Random effect models}

Two models were fitted separately with group and therapist session as a random effect. These models were adjusted for sex, age and baseline outcome.

\section{Longitudinal models (observed data)}

Longitudinal regression techniques enable us to generate a single estimate of treatment over the 12-month period (analogous to using area under the curve analysis to derive a single estimate for the entire time period). The original protocol stated that we would use area under the curve analysis, so this is a deviation in computing method but not in original intention.

Four longitudinal models were fitted with varying covariance-variance structure (compound symmetry, unstructured, heterogeneous, autoregressive heterogeneous). The model with the smallest Akaike's Information Criterion was selected.

Longitudinal models were fitted with fixed effects (age, sex and baseline) and a random effect (group/ therapist).

\section{Numbers needed to treat}

We estimated the number needed to treat (with a 95\% CI) for the primary outcomes at 12 months and the global indicator of change.

This calculation required us to specify the minimally important change for an individual. Although there is no strong consensus, we used the thresholds suggested by previous studies. ${ }^{124,125}$

These were a $30 \%$ change from baseline, i.e. [(baseline-follow-up at 12 months)/baseline] $\times 100$. Here scores changing by $\leq 30 \%$ are considered as deterioration or stability in disability and those $>30 \%$ are improvement. However, owing to rounding errors generated in the method of calculation, the method is imprecise. We also calculated numbers needed to treat based on the global indicator of change distinguishing between those people who reported any improvement versus none or deterioration, and those people who reported stability of symptoms or improvement versus deterioration.

\section{Employment (hours)/days off sick/ number of days off sick}

Change in work status in the last 3 months was summarised using numbers and \% of participants with change in their work status. Hours of employment and days off sick were statistically tested using chi-squared test statistics. Number of days off work (as sick) was analysed using the Wilcoxon signed rank test.

\section{Harm (safety) data}

The number (and percentage) of participants who have a serious adverse event/death/complaint is reported.

\section{Treatment satisfaction and global indicator of change}

We used a seven-point scale to measure satisfaction and global change. ${ }^{99}$ The number of participants (and percentage) within each of the 'satisfaction'/'global change' categories is given for each treatment arm over the course of the study. A chi-squared test for trend was used to assess the difference in treatment effect at each of the three time points.

\section{Subgroup analysis}

The potential biases inherent in undertaking multiple subgroup analyses are well recognised. ${ }^{126}$ However, the BeST trial offers a unique opportunity to generate hypotheses about the profile of participants most likely to benefit from group-based CBA. The most scientifically robust method of subgroup analysis is a test of interaction between treatment and outcome that has been appropriately powered. This can substantially increase sample size requirement. A rough rule is that detection of interactions approximately twice the size of the main effect requires no increase in the sample size, provided that the subgroups are of equal size, the subgroup comparisons are limited and prespecified, and the results are considered hypothesis-generating as opposed to confirmatory. ${ }^{126}$ We report three prespecified analyses alongside the main trial results:

- participants with subacute versus chronic LBP at study entry (duration was entered as a continuous variable, and then in a separate analysis as a binary variable using a cut point of 3 years' duration)

- participants with moderately versus very/ extremely troublesome back pain at study entry

- participants with higher versus lower fear avoidance at study entry $(\leq 14$ or $>14$ on the Fear Avoidance Scale). 
These comparisons assume detection of large effects and that subgroups are of roughly equal size.

\section{Monitoring and time line Trial Steering Committee}

A Trial Steering Committee was responsible for monitoring and supervising the progress of BeST towards its interim and overall objectives.

\section{Data Monitoring and Ethics Committee}

The DMEC was independent of the trial and monitored the ethical, safety and data integrity aspects of the trial. The DMEC determined what analyses were required at each of the meetings and these were supplied by the trial statistician. 



\section{Chapter 4 \\ Results}

\section{Participant approach and characteristics of the randomised sample}

\author{
A total of 9771 people were identified from \\ primary contacts or from primary-care record \\ searches and were sent an initial approach \\ screening questionnaire. Of these people, 3604 \\ returned the screening questionnaire and 3496 \\ provided data that could be used to assess potential \\ eligibility.
}

Of these, 1465 appeared eligible. The most common reasons for ineligibility were that people no longer had back pain $(n=481)$, their back pain was not of sufficient frequency $(n=611)$ or troublesomeness $(n=479)$, or they were pregnant $(n=20)$. Some participants had several reasons for ineligibility.

All 1465 apparently eligible people were invited to attend the first nurse assessment check: 971 attended the first nurse assessment, and of these 833 were eligible for the second nurse assessment; 754 people attended the second nurse assessment and 705 were randomised. Baseline data were not received for four of these and they have been excluded from our analyses.

There were no important differences between those participants who were determined eligible and randomised, and those who were determined eligible and were not randomised. All randomised participants met the eligibility criteria.

The characteristics of the randomised sample are shown in Table 7. In summary, the majority of the randomised sample (70\%) experienced back pain every day. The most common presentation was pain in the back or buttocks, and stiffness and restricted range of motion. Just over half of the randomised sample reported their back pain to be moderately troublesome (55\%), the remainder reporting very or extremely troublesome pain. The average age of the sample was 54 years, and nearly $60 \%$ of participants were women. Most had left full-time education before the age of 15 , and approximately half of the sample reported that they were currently working. If participants were working then they were generally in full-time work (working between 25 and 40 hours a week), were in professional or skilled manual work and were being paid. The majority of those not working were retired.

\section{Description of sample by cluster}

The study recruited from 56 general practices from seven regions across England: Norwich (27.8\% of the sample), Coventry and Solihull (26.8\%), South Warwickshire (18.6\%), Birmingham (12.6\%), Langbaurgh $(7.5 \%)$ and North Warwickshire (6.7\%). The characteristics of each regional sample were generally consistent (Table 8). The only exception was ethnicity. Although overall the sample was representative of the UK population ethnic mix, the regional samples were different in their ethnic make-up. The Birmingham cluster provided the majority of Asian participants. Birmingham also had the greatest proportion of people who were not working.

\section{Success of randomisation}

The severity of back pain was used as a stratification variable along with the regional centre and, as shown in Table 9, the randomisation resulted in a satisfactory balance in the stratification variables over the treatment groups.

\section{Flow and characteristics of randomised participants}

Figure 6 presents the flow diagram for the study. The number of participants randomised and in whom a baseline assessment was received by the trial office was 701 (233 on the AM and 468 on $\mathrm{AM}+\mathrm{CBA})$. An additional four people provided consent and were randomised but no baseline questionnaire was received for them. Hence they have been excluded from the analysis (Figure 6).

In summary, $78 \%$ of the participants provided data at 3 months, $83 \%$ at 6 months and $85 \%$ at 
TABLE 7 Demographic information for those participants who attended the second nurse assessment by randomisation status

\begin{tabular}{|c|c|c|c|}
\hline Details & & $\begin{array}{l}\text { Attended second nurse } \\
\text { assessment }\end{array}$ & Eligible and randomised \\
\hline \multirow[t]{2}{*}{ No. of participants } & Total & 754 & 705 \\
\hline & (with data) & 722 & 701 \\
\hline \multirow[t]{3}{*}{ Age (years) } & Mean (SD) & $54(15)$ & $54(15)$ \\
\hline & Range & $18-85$ & $18-85$ \\
\hline & Missing & 6 & 2 \\
\hline \multirow[t]{3}{*}{ Gender } & Male & $289(40 \%)$ & $279(39 \%)$ \\
\hline & Female & 427 (59\%) & $420(60 \%)$ \\
\hline & Missing $^{\mathrm{a}}$ & $6(1 \%)$ & $2(0 \%)$ \\
\hline \multirow[t]{6}{*}{ Ethnic origin } & White & $632(88 \%)$ & $618(88 \%)$ \\
\hline & Mixed & $8(1 \%)$ & 7 (I\%) \\
\hline & Asian & $29(4 \%)$ & $29(4 \%)$ \\
\hline & Black & $13(2 \%)$ & II (2\%) \\
\hline & Chinese & $2(0 \%)$ & $2(0 \%)$ \\
\hline & Missing $^{\mathrm{a}}$ & $38(5 \%)$ & $34(5 \%)$ \\
\hline \multirow[t]{4}{*}{ Frequency of back pain } & Every day & $487(68 \%)$ & 477 (68\%) \\
\hline & Every day to $3 / 4$ days & $104(14 \%)$ & $102(15 \%)$ \\
\hline & $3 / 4$ to $1 / 2$ a day & $56(8 \%)$ & $56(8 \%)$ \\
\hline & Missinga & $70(10 \%)$ & $66(9 \%)$ \\
\hline \multirow[t]{4}{*}{ Troublesome back } & Not at all & 0 & 0 \\
\hline & Slightly & $7(1 \%)$ & 0 \\
\hline & Moderately & $392(54 \%)$ & $382(55 \%)$ \\
\hline & Very/extremely & $323(45 \%)$ & $319(45 \%)$ \\
\hline \multirow{5}{*}{$\begin{array}{l}\text { Left full-time education } \\
\text { (years) }\end{array}$} & 16 or less & $400(55 \%)$ & 387 (55\%) \\
\hline & $17-19$ & $169(23 \%)$ & $165(23 \%)$ \\
\hline & 20 or over & $113(16 \%)$ & $113(16 \%)$ \\
\hline & Still in further education & $3(0 \%)$ & $3(0 \%)$ \\
\hline & Missing $^{\mathrm{a}}$ & $28(4 \%)$ & $37(5 \%)$ \\
\hline \multirow[t]{3}{*}{ Currently working } & Yes & $303(42 \%)$ & $299(42 \%)$ \\
\hline & No & $302(42 \%)$ & $289(41 \%)$ \\
\hline & Missing $^{\mathrm{a}}$ & 117 (16\%) & 117 (I6\%) \\
\hline
\end{tabular}

12 months. Approximately $1 \%$ of the clinical research forms were returned blank over the course of the trial. A greater amount of the data was collected by telephone follow-up at 12 months (14\%) compared with 3 months (4\%) and 6 months $(8 \%)$. There was no difference in the amount of telephone follow-up between the two arms of the trial at any of the follow-up time points $(p=0.13)$. There was no significant difference in the amount of non-response between treatments over time $(p=0.24 ;$ Table 10).

Just over $5 \%$ (38/701) of participants withdrew over the course of the trial. There was no association between withdrawal and treatment allocation at any of the time points $(p=0.66)$. The usual reason for withdrawal was that participants did not want to complete questionnaires - 15 participants (2\%). 


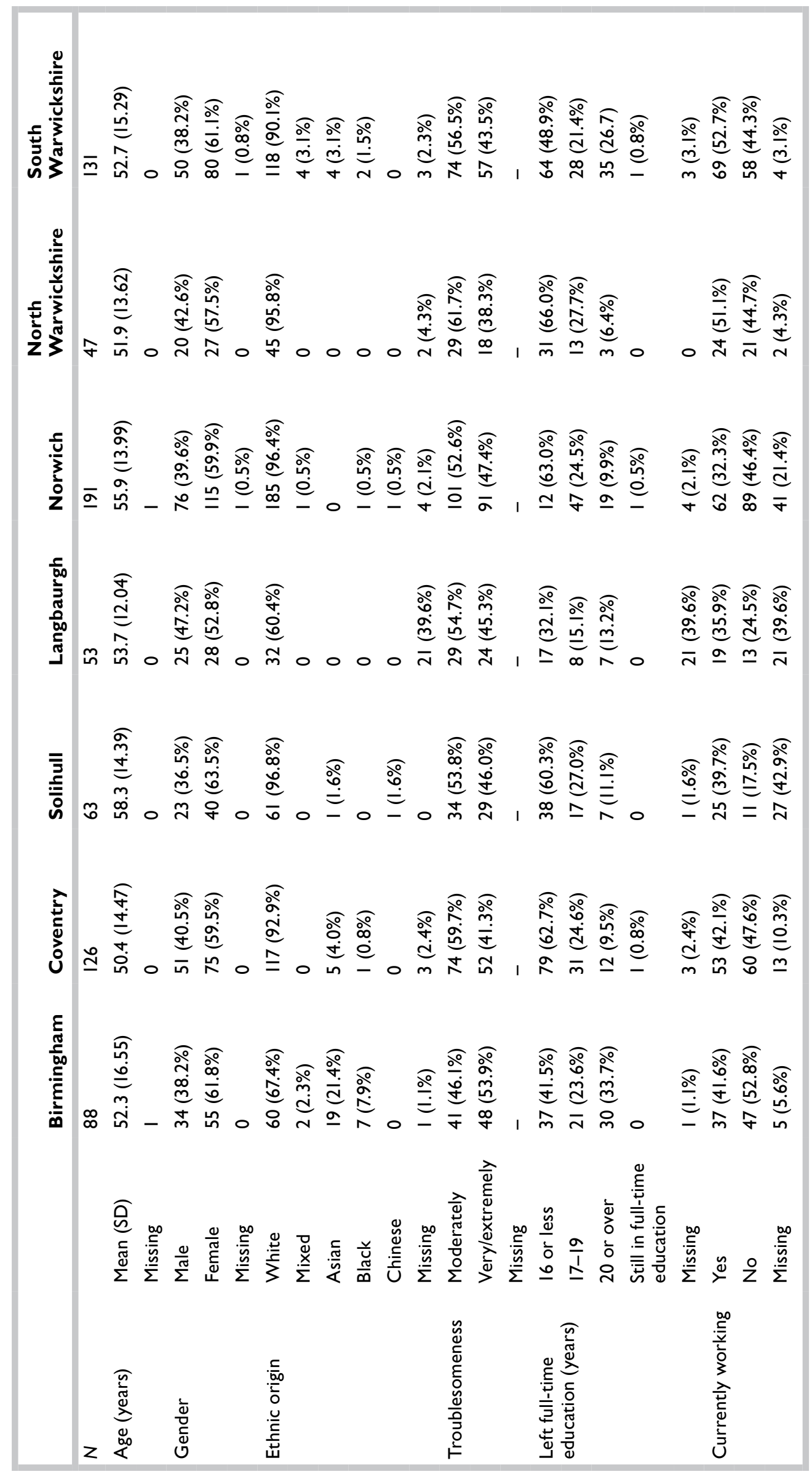


TABLE 9 Number (and percentage) of participants randomised by treatment and regional cluster/severity of back pain

\begin{tabular}{|c|c|c|c|c|}
\hline \multirow[b]{2}{*}{ Cluster } & \multirow[b]{2}{*}{ Severity of pain } & \multicolumn{3}{|c|}{ Treatment } \\
\hline & & AM (\%) & AM+CBA (\%) & Total \\
\hline \multirow[t]{2}{*}{ Birmingham } & Moderate & $14(6)$ & $27(6)$ & $4 \mid(6)$ \\
\hline & Severe & $16(7)$ & $32(7)$ & $48(7)$ \\
\hline \multirow[t]{2}{*}{ Coventry } & Moderate & $23(10)$ & $5 I(I I)$ & $74(\mathrm{II})$ \\
\hline & Severe & $20(9)$ & $32(7)$ & $52(7)$ \\
\hline \multirow[t]{2}{*}{ Solihull } & Moderate & $13(6)$ & $21(5)$ & $34(5)$ \\
\hline & Severe & $7(3)$ & $22(5)$ & $29(4)$ \\
\hline \multirow[t]{2}{*}{ Langbaurgh } & Moderate & II (5) & $18(4)$ & $29(4)$ \\
\hline & Severe & $4(2)$ & $20(4)$ & $24(3)$ \\
\hline \multirow[t]{2}{*}{ Norwich } & Moderate & $34(15)$ & $67(14)$ & $101(14)$ \\
\hline & Severe & $31(13)$ & $60(13)$ & $91(13)$ \\
\hline \multirow[t]{2}{*}{ North Warwickshire } & Moderate & $10(4)$ & $19(4)$ & $29(4)$ \\
\hline & Severe & $6(3)$ & $12(3)$ & $18(3)$ \\
\hline \multirow[t]{2}{*}{ South Warwickshire } & Moderate & $25(\mathrm{II})$ & 49 (II) & $74(\mathrm{II})$ \\
\hline & Severe & $19(8)$ & $38(8)$ & $57(8)$ \\
\hline Total & & 233 & 468 & 701 \\
\hline
\end{tabular}

\section{Missing data and characteristics of the randomised sample}

There was no statistically significant difference in the return rates between the two arms of the trial at any of the follow-up time points $(p=0.67)$. There was no evidence of a systematic difference in the baseline characteristics of participants who provided follow-up data and those who did not (see Appendix 12). The most common pattern of missing data was for data to be missing at the first follow-up point, and then complete for the remainder (Table 11). We obtained some followup data on over $90 \%$ of participants. Reasons for withdrawal are given in Table 12.

The demographic characteristics of all randomised participants (by treatment group) are shown in Table 13. Demographic and baseline characteristics were well balanced across the treatment arms.

\section{Treatments received}

\section{Intervention arm (AM+CBA)}

One participant was randomised to AM but received $\mathrm{CBA}$ in addition to $\mathrm{AM}$.

\section{Details of therapists for cognitive behavioural approach}

We used a variety of methods to recruit the therapists and although we targeted all allied health and nursing professionals, it was mainly physiotherapists who responded. We recruited and trained 19 therapists: 14 physiotherapists, two occupational therapists, one nurse, one clinical psychologist and one health psychologist. In total $77.3 \%(n=378)$ of participants attended groups facilitated by physiotherapists, $6 \%(n=30)$ saw occupational therapists, $9 \%(n=45)$ saw psychologists and $3 \%(n=15)$ saw nurses.

Details of the therapists are shown in Table 14. The therapists had a range of experience with years since professional qualification ranging from 2 to 32 years with a mean of 13.6 years (SD 9.6). Some therapists had experience of running similar groups in the past.

\section{Attendance for assessment and sessions in the trial}

Attendance for the assessment and group sessions is summarised in Table 15. Nearly two-thirds (63\%) of the participants randomised to receive CBA attended the assessment session and at least three of the six group sessions, and were therefore considered to have received the basic elements of 


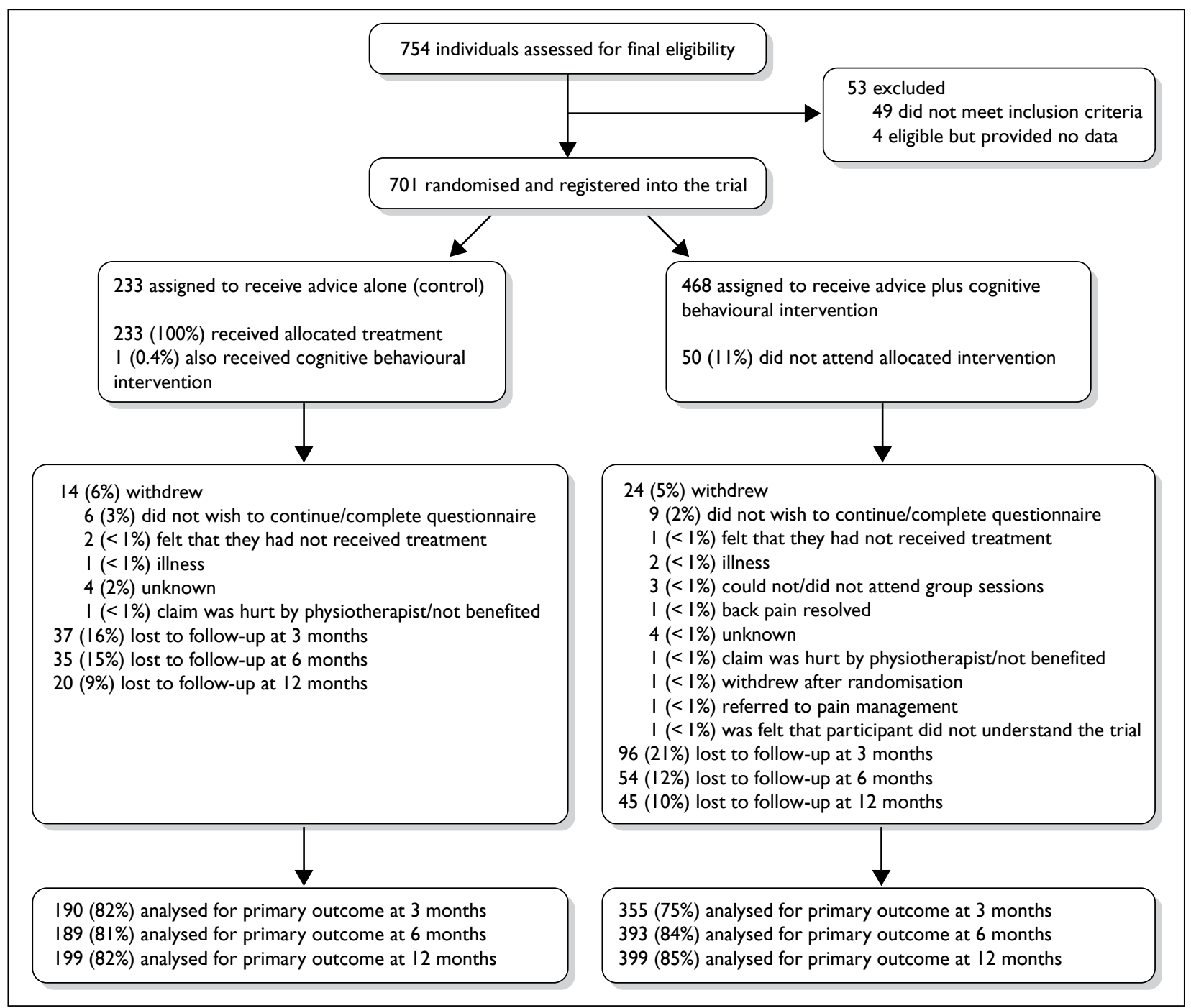

FIGURE 6 Flow chart of the trial.

the treatment. Just over $10 \%$ of people randomised to CBA did not attend any of the programme. Attendance at all six sessions was achieved by $25 \%$ of the sample. There was no evidence to suggest that therapist characteristics were associated with attendance - neither the professional background (Table 16), previous experience with CBA nor years since qualification were associated with attendance rates (Table 15).

There were few differences between the groups of participants who were adherent and those who were not. On average, participants who adhered were 4.5 years older than those who did not $(95 \%$ CI 1.8 to 7.5 ), and had slightly lower pain scores at baseline [MVK (pain) mean difference 4.5, 95\% CI 0.94 to 8.19 ]. There were no differences in RMQ, MVK (disability), sex, troublesomeness of pain or fear avoidance beliefs at baseline.
There were 174 (37\%) participants who did not comply (i.e. had not received three or more groupbased sessions of the therapy) with the AM+CBA therapy.

Therapists were asked to document the reasons for non-attendance if possible. A reason was documented in 80 cases (Table 17).

In addition four participants were excluded from the groups by the therapists (Table 18).

\section{Time from randomisation to starting treatment}

Table 19 provides summary statistics for the time scale of treatment delivery. The target time for attendance at the therapist assessment was set at 45 days after randomisation. Of those participants who attended the assessment, 271 out of $418(65 \%)$ 
TABLE IO Flow of all randomised participants

\begin{tabular}{|c|c|c|c|c|c|}
\hline & & AM (\%) & AM+CBA (\%) & Total (\%) & $p$-value ${ }^{a}$ \\
\hline \multirow{4}{*}{$\begin{array}{l}\text { Number of participants } \\
\text { (with postal questionnaires) }\end{array}$} & Baseline & 233 & 468 & 701 & \multirow[t]{4}{*}{0.67} \\
\hline & Month 3 & $179(77)$ & $335(72)$ & $514(73)$ & \\
\hline & Month 6 & $177(76)$ & $352(75)$ & $529(76)$ & \\
\hline & Month 12 & $161(69)$ & $339(72)$ & $500(7 I)$ & \\
\hline \multirow{4}{*}{$\begin{array}{l}\text { Number of participants } \\
\text { (with core data) }\end{array}$} & Baseline & 0 & 0 & 0 & \multirow[t]{8}{*}{0.13} \\
\hline & Month 3 & II (5) & $20(4)$ & $31(4)$ & \\
\hline & Month 6 & $12(5)$ & $41(9)$ & $53(8)$ & \\
\hline & Month 12 & $38(16)$ & $60(13)$ & $98(14)$ & \\
\hline \multirow{4}{*}{$\begin{array}{l}\text { Total number of participants } \\
\text { (with any data) }\end{array}$} & Baseline & $233(100)$ & $468(100)$ & $701(100)$ & \\
\hline & Month 3 & $190(82)$ & $355(76)$ & $545(78)$ & \\
\hline & Month 6 & $189(8 I)$ & $393(84)$ & $582(83)$ & \\
\hline & Month 12 & $199(85)$ & $399(85)$ & $598(85)$ & \\
\hline \multirow[t]{3}{*}{ Cumulative withdrawals } & Month 3 & $6(3)$ & $17(4)$ & $23(3)$ & \multirow[t]{3}{*}{0.66} \\
\hline & Month 6 & $9(4)$ & $21(5)$ & $30(4)$ & \\
\hline & Month 12 & $14(6)$ & $24(5)$ & $38(5)$ & \\
\hline \multirow[t]{3}{*}{ Lost to follow-up (discrete) } & Month 3 & $34(15)$ & $83(18)$ & $117(17)$ & \multirow[t]{9}{*}{0.24} \\
\hline & Month 6 & $30(13)$ & $44(9)$ & $74(\mathrm{II})$ & \\
\hline & Month 12 & $19(8)$ & $42(9)$ & $61(9)$ & \\
\hline \multirow[t]{3}{*}{ Returned blank } & Month 3 & I (0.4) & $6(I)$ & $7(\mathrm{I})$ & \\
\hline & Month 6 & $3(I)$ & $5(I)$ & $8(I)$ & \\
\hline & Month 12 & I (0.4) & $3(0.6)$ & $4(0.6)$ & \\
\hline \multirow[t]{3}{*}{ Other reasons for no data } & Month 3 & $2(I)$ & $7(2)$ & $9(1)$ & \\
\hline & Month 6 & $2(1)$ & $5(I)$ & $7(\mathrm{I})$ & \\
\hline & Month 12 & 0 & 0 & 0 & \\
\hline
\end{tabular}

received their assessment within the target time and $313(75 \%)$ had completed the group treatment by the 3-month follow-up time point (Table 19).

Meeting the target time proved more difficult to achieve for some of the groups. This was mainly because of delays at one site: $16 \%$ of participants at this site were assessed before 45 days compared with an average of $80 \%$ across the other sites (Figure 7). There did not appear to be pressures on venue or staff time at this site but there were consistent delays in assessing participants and starting groups. A minority of other groups were delayed by last minute changes in therapist availability.

Thirteen participants attended a group at a later date than the first one they were randomised to due to changes in their personal circumstances after randomisation. No participants switched to a different group once sessions had commenced. There was no change-over in therapist during any of the 6 -week group sessions. The attendance rate for those who started the group in less than 45 days was the same as for those who waited longer than 45 days (71\% and $70 \%$ respectively).

\section{Number and size of groups}

Group size was difficult to standardise because of variations in recruitment rate. In total, 62 groups were run with a mean size of eight (SD 1.62, range 4-12). A breakdown of the group sizes and attendance rates is given in Table 20. Group size was not associated with attendance rate $(p=0.17)$.

\section{Group intervention venue and time}

During the trial, a variety of premises were used and a range of times were offered to cater for the needs of the participants. The venue setting did not impact on attendance compliance, with $63 \%$ 
TABLE II Tracking of participants longitudinally who returned clinical research forms/core outcomes

\begin{tabular}{lllc}
\hline months & $\mathbf{6}$ months & $\mathbf{1 2}$ months & Total (\%) \\
\hline Present & Present & Present & $492(70.2)$ \\
Present & Present & Absent & $18(2.6)$ \\
Present & Absent & Absent & $21(3.0)$ \\
Present & Absent & Present & $14(2.0)$ \\
Absent & Present & Present & $58(8.3)$ \\
Absent & Absent & Present & $34(4.9)$ \\
Absent & Present & Absent & $14(2.0)$ \\
Absent & Absent & Absent & $50(7.1)$
\end{tabular}

TABLE 12 Reasons for withdrawals in each arm

\begin{tabular}{|c|c|c|c|}
\hline & AM (\%) & AM+CBA (\%) & Total (\%) \\
\hline Did not wish to continue/complete questionnaires & $6(3)$ & $9(2)$ & $15(2)$ \\
\hline Felt they did not receive treatment & $2(I)$ & $\mathrm{I}(0.2)$ & $3(0.4)$ \\
\hline Illness & $\mathrm{I}(0.4)$ & $2(0.4)$ & $3(0.4)$ \\
\hline Could not/did not attend groups & 0 & $3(0.6)$ & $3(0.6)$ \\
\hline Back pain resolved & 0 & $\mathrm{I}(0.2)$ & $\mathrm{I}(0.1)$ \\
\hline Unknown & $4(2)$ & $4(1)$ & $8(1)$ \\
\hline Withdrew after randomisation & 0 & $\mathrm{I}(0.2)$ & $\mathrm{I}(0.1)$ \\
\hline Claims was hurt by physiotherapist/not benefiting & $\mathrm{I}(0.4)$ & $\mathrm{I}(0.2)$ & $2(0.3)$ \\
\hline Referred to pain management & 0 & $\mathrm{I}(0.2)$ & $\mathrm{I}(0.1)$ \\
\hline Was felt participant did not understand the trial & 0 & I $(0.2)$ & $\mathrm{I}(0.1)$ \\
\hline Deaths & 0 & $\mathrm{I}(0.2)$ & $\mathrm{I}(0.2)$ \\
\hline Withdrawals (total) & $14(6)$ & $25(5)$ & $39(6)$ \\
\hline
\end{tabular}

TABLE I3 Demographic characteristics for all randomised participants by treatment

$\begin{array}{llcc}\text { Details } & & \text { AM } & \text { AM+CBA } \\ \text { Age (years) } & \text { Mean (SD) } & 233 & 466 \\ & \text { Range } & 54(15) & 53(15) \\ \text { Missing } & 19-85 & 18-85 & 2 \\ \text { Gender } & \text { Male } & - & 189(40 \%) \\ & \text { Female } & 90(39 \%) & 278(59 \%) \\ \text { Ethnic origin } & \text { Missing } & 142(61 \%) & 1(0 \%) \\ & \text { White } & 1(0 \%) & 412(88 \%) \\ & \text { Mixed } & 206(88 \%) & 4(1 \%) \\ \text { Frequency of back pain } & 3(1 \%) & 21(5 \%) \\ & \text { Alack } & 8(3 \%) & 7(2 \%) \\ & \text { Chinese } & 4(2 \%) & 1(0 \%) \\ & \text { Other } & 1(0 \%) & 23(5 \%) \\ & \text { Every day } & 11(5 \%) & 315(67 \%) \\ & \text { Every day to } 3 / 4 \text { days } & 162(70 \%) & 71(15 \%)\end{array}$


TABLE I3 Demographic characteristics for all randomised participants by treatment (continued)

\begin{tabular}{|c|c|c|c|}
\hline Details & & AM & $A M+C B A$ \\
\hline \multirow[t]{3}{*}{ Back pain troublesomeness } & Moderately & $130(56 \%)$ & $252(54 \%)$ \\
\hline & Very/extremely & 103 (44\%) & $216(46 \%)$ \\
\hline & Missing & 0 & 0 \\
\hline \multirow[t]{5}{*}{ Left full-time education (years) } & 16 or less & $12 \mid(52 \%)$ & $266(57 \%)$ \\
\hline & $17-19$ & 61 (26\%) & $104(22 \%)$ \\
\hline & 20 or over & $40(17 \%)$ & $73(16 \%)$ \\
\hline & Still in full-time education & $2(1 \%)$ & I (0\%) \\
\hline & Missing & $9(4 \%)$ & $24(5 \%)$ \\
\hline \multirow{5}{*}{$\begin{array}{l}\text { Duration of back pain (pain first } \\
\text { ever started, years ago) }\end{array}$} & $N$ & 226 & 446 \\
\hline & Mean & $13(13)$ & $13(13)$ \\
\hline & Median & 9 & 10 \\
\hline & Range & $0.25-53.0$ & $0.12-84.0$ \\
\hline & Missing & 7 & 22 \\
\hline \multirow[t]{5}{*}{ Back pain } & Comes and goes over time & $53(23 \%)$ & $108(23 \%)$ \\
\hline & Fairly constant & 122 (52\%) & $250(53 \%)$ \\
\hline & Getting worse & $55(24 \%)$ & $104(22 \%)$ \\
\hline & Getting better & $\mathrm{I}(0 \%)$ & $4(1 \%)$ \\
\hline & Missing & $2(1 \%)$ & $2(0 \%)$ \\
\hline \multirow{3}{*}{$\begin{array}{l}\text { Received treatment for back pain } \\
\text { in last } 6 \text { months }\end{array}$} & Yes & $80(34 \%)$ & 197 (42\%) \\
\hline & No & $15 \mid$ (65\%) & $269(58 \%)$ \\
\hline & Missing & $2(1 \%)$ & $2(0 \%)$ \\
\hline \multirow{3}{*}{$\begin{array}{l}\text { Received benefit payments (of } \\
\text { any type) }\end{array}$} & Yes & 97 (42\%) & $180(39 \%)$ \\
\hline & No & I35 (58\%) & $281(60 \%)$ \\
\hline & Missing & $\mathrm{I}(0 \%)$ & $7(2 \%)$ \\
\hline \multirow[t]{4}{*}{ Currently working } & Yes, full time & $7 \mid(31 \%)$ & 156 (33\%) \\
\hline & Yes, part time & $39(17 \%)$ & $82(18 \%)$ \\
\hline & No & 122 (52\%) & $228(49 \%)$ \\
\hline & Missing & $\mathrm{I}(0 \%)$ & $2(0 \%)$ \\
\hline
\end{tabular}

attendance for both PCT premises and private community premises. The day and time of the group did impact on compliance. Groups run on a Friday had an attendance of $70 \%$ compared with around $61 \%$ on other days. Groups running around lunchtime and after work saw the best compliance, with $68 \%$ and $65 \%$ respectively. Evening groups were surprisingly poorly attended (51\%) despite being requested by participants.

Thirty-five group sessions (56\% of all groups run) were assessed for quality assurance either with an assessor in the session or via audio recordings. The results are shown in Table 21 .

As many of the therapists were new to the CB skills of questioning, agenda setting, eliciting beliefs and feedback we expected these items to be more difficult to achieve, and this was reflected in the quality assurance scores. The remaining skills were evidenced in most groups.

\section{Description of treatment effectiveness}

Data summaries by treatment group are given in Table 22 for the primary outcomes and in Tables 23 and 24 for the secondary outcomes.

\section{Therapist and group effects}

The ICCs were very small and there was no evidence of significant clustering effects related either to group sessions or to therapist (Table 25), the implication being that group session and therapist effects were negligible. Estimates of the 


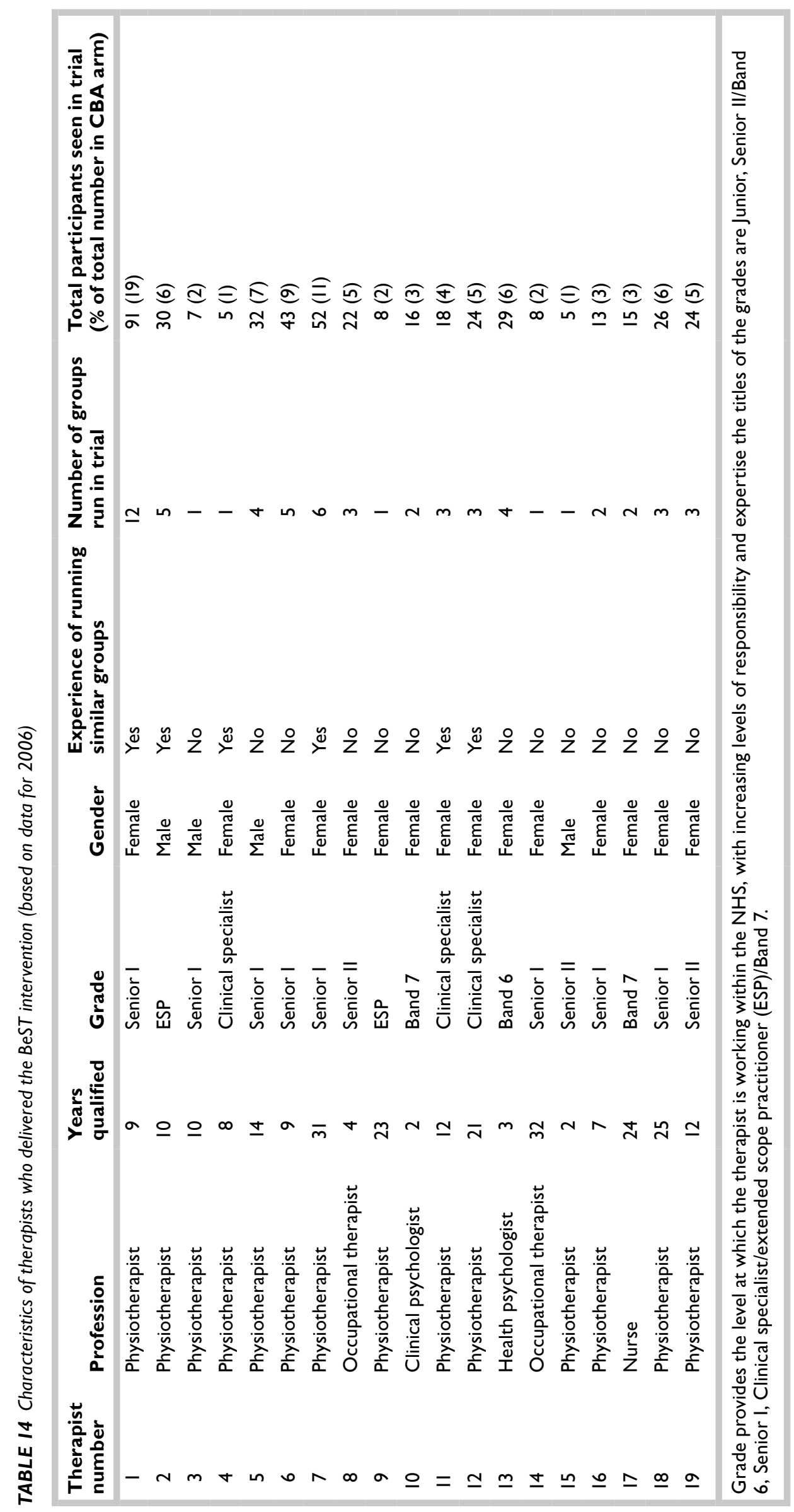


TABLE I5 Number (and percentage) of participants attending the assessment and group sessions

\begin{tabular}{lc} 
& No. (\%) of participants \\
Did not attend for assessment & $50(\mathrm{II})$ \\
Attended assessment & $418(89)$ \\
Attended assessment plus $\geq 1$ session & $358(77)$ \\
Attended assessment plus $\geq 2$ sessions & $325(69)$ \\
Attended assessment plus $\geq 3$ sessions & $294(63)$ \\
Attended assessment plus $\geq 4$ sessions & $275(59)$ \\
Attended assessment plus $\geq 5$ sessions & $225(48)$ \\
Attended assessment plus $\geq 6$ sessions & $121(26)$ \\
\hline The number of sessions attended does not imply consecutive sessions.
\end{tabular}

TABLE 16 Number (and percentage) of patients seen by each therapist profession with attendance rates

\begin{tabular}{lccr} 
Profession & $\begin{array}{l}\text { Attended <3 group } \\
\text { sessions (\%) }\end{array}$ & $\begin{array}{l}\text { Attended } \geq \mathbf{3} \text { group } \\
\text { sessions (\%) }\end{array}$ & Total (\%) \\
\hline Physiotherapists & $136(36)$ & $242(64)$ & $378(100)$ \\
Occupational therapists & II (37) & $19(63)$ & $30(100)$ \\
Nurses & $7(47)$ & $8(53)$ & $15(100)$ \\
Psychologists & $20(44)$ & $25(56)$ & $45(100)$
\end{tabular}

TABLE 17 Reasons provided by participants for not attending

$\begin{array}{ll} & \text { No. of participants (\%) } \\ \text { Unknown } & 95(54) \\ \text { Unwell } & 18(10) \\ \text { Changes to work } & 16(9) \\ \text { Family issues } & 15(9) \\ \text { Decided format of group 'not for them' } & 10(6) \\ \text { Difficulty attending - other } & 10(6) \\ \text { Decided no benefit to intervention } & 4(2) \\ \text { Moved } & 3(2)\end{array}$

TABLE 18 Reasons provided by the therapists for excluding the four participants from the group

\begin{tabular}{ll} 
Therapist & Reason \\
\hline 8 & $\begin{array}{l}\text { Patient has other musculoskeletal disorders and also very resistant to change. Participant and therapist } \\
\text { agreed that the group was not for him }\end{array}$ \\
6 & Wife aggressive to therapist and wanted to attend sessions. Participant had learning difficulties \\
7 & Excluded after randomisation by GP \\
5 & Excluded as participant was receiving private physiotherapy
\end{tabular}


TABLE 19 Time from randomisation to assessment, the first group session and attendance at last CB group

\begin{tabular}{lllll}
\hline & $\begin{array}{l}\text { No. of days from } \\
\text { randomisation to } \\
\text { assessment }\end{array}$ & $\begin{array}{l}\text { No. of days from } \\
\text { assessment to } \\
\text { session one }\end{array}$ & $\begin{array}{l}\text { No. of days from } \\
\text { randomisation to } \\
\text { session one }\end{array}$ & $\begin{array}{l}\text { No. of days from } \\
\text { randomisation to last } \\
\text { CB session attended }\end{array}$ \\
Mean & 25.7 & 17.7 & 43.1 & 82.1 \\
Median & 21.0 & 14.0 & 38.0 & 77.0 \\
SD & 22.3 & 13.7 & 26.0 & 26.4
\end{tabular}

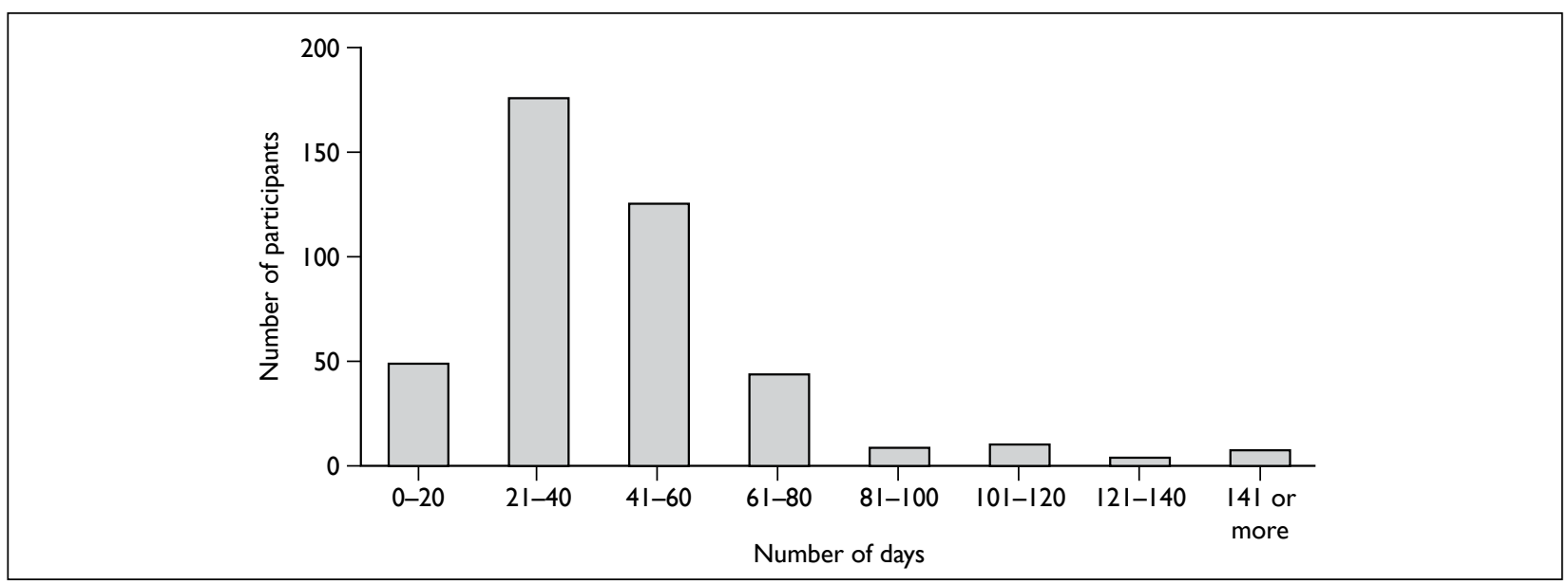

FIGURE 7 Number of days from randomisation to receiving assessment.

TABLE 20 Group size with number of patients and attendance rates at group sessions

\begin{tabular}{cccc|} 
Group size & $\begin{array}{l}\text { No. of groups run at this } \\
\text { size }\end{array}$ & Total participants & $\begin{array}{l}\text { Attended } \geq 3 \text { group sessions } \\
(\%)\end{array}$ \\
\hline 4 & 2 & 8 & $6(75)$ \\
5 & 3 & 15 & $10(67)$ \\
6 & 11 & 66 & $44(67)$ \\
7 & 14 & 98 & $60(61)$ \\
8 & 14 & 112 & $70(64)$ \\
9 & 10 & 90 & $52(58)$ \\
10 & 6 & 60 & $40(68)$ \\
11 & 1 & 11 & $5(46)$ \\
12 & 1 & 12 & $7(64)$ \\
\hline
\end{tabular}


TABLE 2 I Quality assurance tool with percentage achieved during visits or via recordings

\begin{tabular}{|llccl}
\hline Item & Not achieved & $\begin{array}{l}\text { Partially achieved } \\
\text { (\% of total) }\end{array}$ & $\begin{array}{l}\text { Satisfactorily } \\
\text { achieved (\% of total) }\end{array}$ \\
\hline Content & Set agenda & $5(14)$ & $8(23)$ & $22(63)$ \\
& Homework reviewed & $0(0)$ & $1(3)$ & $34(97)$ \\
& Topics covered & $0(0)$ & $3(9)$ & $32(91)$ \\
& Break & $0(0)$ & $0(0)$ & $35(100)$ \\
& Homework set & $0(0)$ & $5(14)$ & $30(86)$ \\
Style & Feedback elicited & $6(17)$ & $7(20)$ & $22(63)$ \\
& Exercises checked in break & $0(0)$ & $1(3)$ & $34(97)$ \\
& Encouraged group participation & $0(0)$ & $4(11)$ & $31(89)$ \\
& Listened appropriately & $0(0)$ & $0(0)$ & $35(100)$ \\
& Empathy demonstrated & $2(6)$ & $1(3)$ & $32(91)$ \\
& Elicited beliefs/thoughts & $2(6)$ & $11(31)$ & $22(63)$ \\
& Questioning style demonstrated & $2(6)$ & $8(23)$ & $25(71)$ \\
& Referred to CB model & $4(11)$ & $4(11)$ & $27(77)$ \\
& Appropriate pacing of session & $1(3)$ & $7(20)$ & $27(77)$ \\
& Appeared professional & $0(0)$ & $0(0)$ & $35(100)$ \\
& Comfortable & $0(0)$ & $1(3)$ & $34(97)$ \\
& Spacious & $0(0)$ & $0(0)$ & $35(100)$
\end{tabular}

TABLE 22 Summary statistics for the primary clinical outcome measures at each follow-up time point by treatment (unadjusted)

\begin{tabular}{|c|c|c|c|c|c|c|c|}
\hline & & \multicolumn{2}{|l|}{ RMQ } & \multicolumn{2}{|c|}{ MVK (disability) } & \multicolumn{2}{|c|}{ MVK (pain) } \\
\hline & & AM & $A M+C B A$ & AM & AM+CBA & AM & $A M+C B A$ \\
\hline \multirow[t]{5}{*}{ Baseline } & $N$ & 232 & 468 & 228 & 455 & 230 & 463 \\
\hline & Mean & 8.5 & 8.8 & 46.2 & 48.5 & 59.4 & 59.3 \\
\hline & SD & 4.72 & 4.99 & 23.79 & 23.85 & 19.51 & 19.24 \\
\hline & Median & 7.5 & 8.0 & 46.7 & 50.0 & 60.0 & 56.7 \\
\hline & Range & $0-22$ & $0-23$ & $0-100$ & $0-100$ & $10-100$ & $6.67-100$ \\
\hline \multirow[t]{5}{*}{ Month 3} & $N$ & 179 & 335 & 187 & 345 & 189 & 354 \\
\hline & Mean & 7.3 & 6.5 & 38.8 & 35.3 & 52.9 & 46.76 \\
\hline & SD & 5.28 & 5.27 & 24.68 & 24.33 & 22.74 & 23.13 \\
\hline & Median & 6.0 & 5.0 & 36.7 & 30.0 & 53.3 & 46.7 \\
\hline & Range & $0-24$ & $0-24$ & $0-100$ & $0-96.67$ & $0-100$ & $0-100$ \\
\hline \multirow[t]{5}{*}{ Month 6} & $N$ & 177 & 352 & 183 & 381 & 187 & 387 \\
\hline & Mean & 7.4 & 6.1 & 41.8 & 33.9 & 53.0 & 44.5 \\
\hline & SD & 5.34 & 5.44 & 24.72 & 25.94 & 23.29 & 25.16 \\
\hline & Median & 6.0 & 4.0 & 43.3 & 30.0 & 53.3 & 43.3 \\
\hline & Range & $0-23$ & $0-24$ & $0-100$ & $0-100$ & $0-100$ & $0-100$ \\
\hline \multirow[t]{5}{*}{ Month 12} & $N$ & 159 & 339 & 189 & 374 & 195 & 392 \\
\hline & Mean & 6.9 & 6.1 & 40.0 & 33.3 & 51.0 & 44.5 \\
\hline & $S D$ & 5.12 & 5.62 & 25.22 & 26.22 & 23.93 & 26.04 \\
\hline & Median & 6.0 & 4.0 & 40.0 & 26.7 & 50.0 & 46.7 \\
\hline & Range & $0-22$ & $0-24$ & $0-100$ & $0-100$ & $3.33-100$ & $0-100$ \\
\hline
\end{tabular}

MVK, Modified Von Korff Scale; RMQ, Roland Morris Disability Questionnaire. 
TABLE 23 Summary statistics for the health-related quality of life outcome measures at each follow-up time point and treatment

\begin{tabular}{|c|c|c|c|c|c|}
\hline & & \multicolumn{2}{|c|}{ SF-I2 (physical) } & \multicolumn{2}{|c|}{ SF-I2 (mental) } \\
\hline & & AM & $A M+C B A$ & AM & $A M+C B A$ \\
\hline \multirow[t]{5}{*}{ Baseline } & $N$ & 217 & 421 & 217 & 421 \\
\hline & Mean & 46.1 & 44.5 & 37.5 & 37.1 \\
\hline & $S D$ & 11.03 & $|I .5|$ & 10.08 & 9.31 \\
\hline & Median & 46.4 & 43.9 & 37.4 & 37.3 \\
\hline & Range & $17-73$ & $11-69$ & $|2-6|$ & $7-62$ \\
\hline \multirow[t]{5}{*}{ Month 3} & $N$ & 176 & 332 & 176 & 332 \\
\hline & Mean & 46.4 & 47.0 & 39.1 & 40.7 \\
\hline & SD & 11.25 & $1 \mathrm{I} .44$ & 10.38 & 11.05 \\
\hline & Median & 47.6 & 48.9 & 39.8 & 42.4 \\
\hline & Range & $12-68$ & II-7I & $11-62$ & $7-61$ \\
\hline \multirow[t]{5}{*}{ Month 6} & $N$ & 177 & 362 & 177 & 362 \\
\hline & Mean & 46.1 & 47.6 & 39.2 & 41.0 \\
\hline & SD & 10.61 & 11.45 & 10.33 & 11.26 \\
\hline & Median & 47.0 & 49.6 & 39.7 & 41.7 \\
\hline & Range & $17-66$ & $|I-7|$ & $10-69$ & $7-68$ \\
\hline \multirow[t]{5}{*}{ Month 12} & $N$ & 187 & 375 & 187 & 375 \\
\hline & Mean & 47.0 & 46.4 & 38.6 & 41.9 \\
\hline & SD & 11.35 & 11.51 & $|I .3|$ & 11.77 \\
\hline & Median & 47.9 & 48.3 & 39.3 & 43.5 \\
\hline & Range & $12-68$ & $|3-7|$ & $13-63$ & $7-70$ \\
\hline
\end{tabular}

treatment effects were not altered by the inclusion of therapist or group effects and hence results are based on the linear models adjusted for age, sex and the baseline value of the variable being tested. Within the adjusted models, only age and baseline measures were statistically significant. This was consistent across all the outcomes and time points.

Adjusted effects are given in Table 26. Both tables provide the estimate of difference in change between the intervention groups with a $95 \%$ CI.

\section{Primary outcomes}

\section{Back pain related disability - Roland} Morris Disability Questionnaire

Figure 8 shows the change from baseline in the RMQ for each of the follow-up time points by treatment arm. Improvements in LBP-related disability occurred in the CBA and AM arms of the trial, but were of different magnitude and time course. Improvements in the AM arm were on average 1.1 RMQ points, with change occurring between baseline and 3 months and no further change thereafter. The change in the CBA arm was almost double that of the AM arm by 3 months, and the treatment difference continued to widen at 6 and 12 months.

The difference between the treatment arms was estimated to be, on average, 1.1 RMQ points at 3 months, rising to 1.4 and 1.3 RMQ points at 6 and 12 months respectively. These differences between treatments were all statistically significant.

\section{Back disability - Modified Von Korff Scale (disability)}

Figure 9 shows the change in MVK (disability) by treatment arm. Both treatment arms showed improvements over baseline, but these were of different magnitude and time course. The AM group improved between 0 and 3 months, and declined thereafter. The CBA group showed a greater improvement. At 6 and 12 months the difference between CBA and AM was more than twofold. 
TABLE 24 Summary statistics for the secondary clinical outcome measures at each follow-up time point and treatment

\begin{tabular}{|c|c|c|c|c|c|}
\hline & & \multicolumn{2}{|c|}{ FABQ } & \multicolumn{2}{|c|}{ Pain self-efficacy } \\
\hline & & AM & $A M+C B A$ & AM & $A M+C B A$ \\
\hline \multirow[t]{5}{*}{ Baseline } & $N$ & 219 & 443 & 223 & 453 \\
\hline & Mean & 14.2 & 13.6 & 41.2 & 39.6 \\
\hline & SD & 6.22 & 6.34 & 12.54 & 13.39 \\
\hline & Median & 15.0 & 14.0 & 43.0 & 41.0 \\
\hline & Range & $0-24$ & $0-24$ & $5-60$ & $2-60$ \\
\hline \multirow[t]{5}{*}{ Month 3} & $N$ & 173 & 319 & 173 & 325 \\
\hline & Mean & 13.3 & 10.3 & 40.6 & 43.0 \\
\hline & SD & 6.07 & 6.37 & $|3.5|$ & $13.3 \mid$ \\
\hline & Median & 14.0 & 11.0 & 43.0 & 46.0 \\
\hline & Range & $0-24$ & $0-24$ & $3-60$ & $2-60$ \\
\hline \multirow[t]{5}{*}{ Month 6} & $N$ & 168 & 336 & 170 & 333 \\
\hline & Mean & 13.8 & 10.4 & 40.4 & 43.3 \\
\hline & SD & 6.09 & 6.54 & 13.38 & 13.54 \\
\hline & Median & 14.0 & 10.5 & 42.0 & 46.0 \\
\hline & Range & $0-24$ & $0-24$ & $2-60$ & $3-60$ \\
\hline \multirow[t]{5}{*}{ Month 12} & $N$ & 152 & 320 & 155 & 317 \\
\hline & Mean & 13.1 & 9.9 & 41.3 & 43.2 \\
\hline & SD & 6.18 & 6.26 & 13.28 & 14.08 \\
\hline & Median & 14.0 & 10.0 & 43.0 & 47.0 \\
\hline & Range & $0-24$ & $0-24$ & $2-60$ & $4-60$ \\
\hline
\end{tabular}

TABLE 25 Intracluster correlation coefficient for RMQ score and MVK questionnaire (using change from baseline)

\begin{tabular}{|c|c|c|c|c|c|c|c|}
\hline \multirow[b]{2}{*}{ Cluster } & \multirow[b]{2}{*}{ Month } & \multicolumn{2}{|l|}{ RMQ } & \multicolumn{2}{|c|}{ MVK (disability) } & \multicolumn{2}{|c|}{ MVK (pain) } \\
\hline & & ICC & $95 \% \mathrm{Cl}$ & ICC & $95 \% \mathrm{Cl}$ & ICC & $95 \% \mathrm{Cl}$ \\
\hline \multirow{3}{*}{$\begin{array}{l}\text { Group } \\
\text { session }\end{array}$} & 3 & -0.01 & -0.02 to 0.001 & -0.02 & -0.04 to -0.02 & 0.01 & -0.02 to 0.03 \\
\hline & 6 & 0.09 & -0.02 to 0.19 & -0.04 & -0.06 to -0.01 & -0.02 & -0.03 to -0.02 \\
\hline & 12 & -0.002 & -0.02 to 0.02 & -0.02 & -0.02 to -0.02 & 0.02 & -0.02 to 0.06 \\
\hline \multirow[t]{3}{*}{ Therapist } & 3 & 0.01 & -0.04 to 0.05 & -0.02 & -0.04 to 0.01 & 0.01 & -0.03 to 0.05 \\
\hline & 6 & 0.03 & -0.02 to 0.09 & -0.004 & -0.04 to 0.03 & -0.001 & -0.03 to 0.03 \\
\hline & 12 & -0.0001 & -0.04 to 0.04 & -0.002 & -0.04 to 0.03 & -0.003 & -0.03 to 0.03 \\
\hline
\end{tabular}

The difference between CBA and AM was estimated to be on average $4.3 \%$ at 3 months, $8.1 \%$ at 6 months and $8.4 \%$ at 12 months. The differences between treatments were statistically significant at all time points.

\section{Back disability - Modified Von Korff Scale (pain)}

Figure 10 shows the change in the MVK (pain) by treatment arm. Both treatment arms showed improvements over baseline, but these were of different magnitude and time course. Pain levels 
TABLE 26 Clinical outcome measures (using change from baseline) - adjusted (for age, sex and baseline) linear regression models analysis

\begin{tabular}{|c|c|c|c|c|c|}
\hline & \multirow[b]{2}{*}{ Period } & \multirow[b]{2}{*}{$\mathbf{N}(\%)$} & \multicolumn{2}{|c|}{ Mean difference between treatments } & \multirow[b]{2}{*}{$p$-value } \\
\hline & & & Mean & $95 \% \mathrm{Cl}$ & \\
\hline \multirow[t]{4}{*}{ RMQ } & 3 & $513(73.2)$ & -1.1 & -1.74 to -0.37 & 0.003 \\
\hline & 6 & $528(75.3)$ & -1.4 & -2.14 to -0.72 & $<0.0001$ \\
\hline & 12 & $498(71.0)$ & -1.3 & -2.05 to -0.55 & 0.0008 \\
\hline & Longitudinal & $1539(73.2)$ & -1.2 & -1.93 to 0.39 & 0.0001 \\
\hline \multirow[t]{4}{*}{ MVK (disability) } & 3 & $519(74.0)$ & -4.3 & -8.20 to -0.42 & 0.03 \\
\hline & 6 & $551(78.6)$ & -8.1 & -12.03 to -4.09 & $<0.0001$ \\
\hline & 12 & $552(78.7)$ & -8.4 & -12.39 to -4.35 & $<0.0001$ \\
\hline & Longitudinal & $1622(77.1)$ & -6.5 & -10.54 to -2.48 & $<0.0001$ \\
\hline \multirow[t]{4}{*}{ MVK (pain) } & 3 & $538(76.7)$ & -6.8 & -10.18 to -3.46 & $<0.0001$ \\
\hline & 6 & $569(8 \mathrm{I} .2)$ & -8.0 & -11.73 to -4.30 & $<0.0001$ \\
\hline & 12 & $583(83.2)$ & -7.0 & -10.66 to -3.24 & 0.0003 \\
\hline & Longitudinal & $1690(80.4)$ & -6.7 & -10.44 to -2.98 & $<0.0001$ \\
\hline \multirow[t]{4}{*}{ SF-I2 (physical) } & 3 & $470(67.0)$ & 2.2 & 0.72 to 3.68 & 0.004 \\
\hline & 6 & 497 (70.9) & 1.8 & 0.34 to 3.25 & 0.016 \\
\hline & 12 & $521(74.3)$ & 4.1 & 2.56 to 5.57 & $<0.0001$ \\
\hline & Longitudinal & $1488(70.8)$ & 4.1 & 2.08 to 6.04 & $<0.0001$ \\
\hline \multirow[t]{4}{*}{ SF-12 (mental) } & 3 & $470(67.0)$ & 1.3 & -0.37 to 2.96 & 0.129 \\
\hline & 6 & 497 (70.9) & 2.5 & 0.78 to 4.26 & 0.005 \\
\hline & 12 & $521(74.3)$ & 0.1 & -1.62 to 1.80 & 0.91 \\
\hline & Longitudinal & $1488(70.8)$ & 0.1 & -2.16 to 2.34 & 0.91 \\
\hline \multirow[t]{4}{*}{ FABQ (physical activity) } & 3 & $47 I(67.2)$ & -2.6 & -3.64 to -1.63 & $<0.0001$ \\
\hline & 6 & $480(68.5)$ & -3.1 & -4.15 to -2.05 & $<0.0001$ \\
\hline & 12 & $447(63.7)$ & -3.0 & -4.08 to -1.88 & $<0.0001$ \\
\hline & Longitudinal & $1398(66.5)$ & -2.8 & -3.98 to -1.73 & $<0.0001$ \\
\hline \multirow[t]{4}{*}{ Pain self-efficacy } & 3 & $483(68.9)$ & 3.2 & 1.34 to 4.93 & 0.0007 \\
\hline & 6 & $490(69.9)$ & 4.1 & 2.30 to 5.98 & $<0.0001$ \\
\hline & 12 & $456(65.0)$ & 3.8 & 1.93 to 5.67 & $<0.0001$ \\
\hline & Longitudinal & $1429(68.0)$ & 3.9 & 1.94 to 5.85 & $<0.0001$ \\
\hline
\end{tabular}

in the AM group improved gradually over the 12-month period. Improvements in pain were more than double in the CBA arm at 3 months, and pain continued to improve (although at a slower rate) between 3, 6 and 12 months. The effect on pain peaked at 12 months. These differences between treatments were statistically significant at all time points.

The difference between CBA and AM was estimated to be on average $6.8 \%$ at 3 months, $8 \%$ at 6 months and $7 \%$ at 12 months.
On average, a participant improved twice as much on the AM+CBA arm as on AM alone.

\section{Secondary and intermediary outcomes Short Form-12 physical subscale}

Figure 11 shows the change in the SF-12 physical subscale by treatment arm. Both treatment arms showed improvements over baseline in the first 6 months of follow-up, but these were of different magnitude and time course. Improvements in the AM arm were very small, and by 12 months there was no difference in comparison with baseline. 


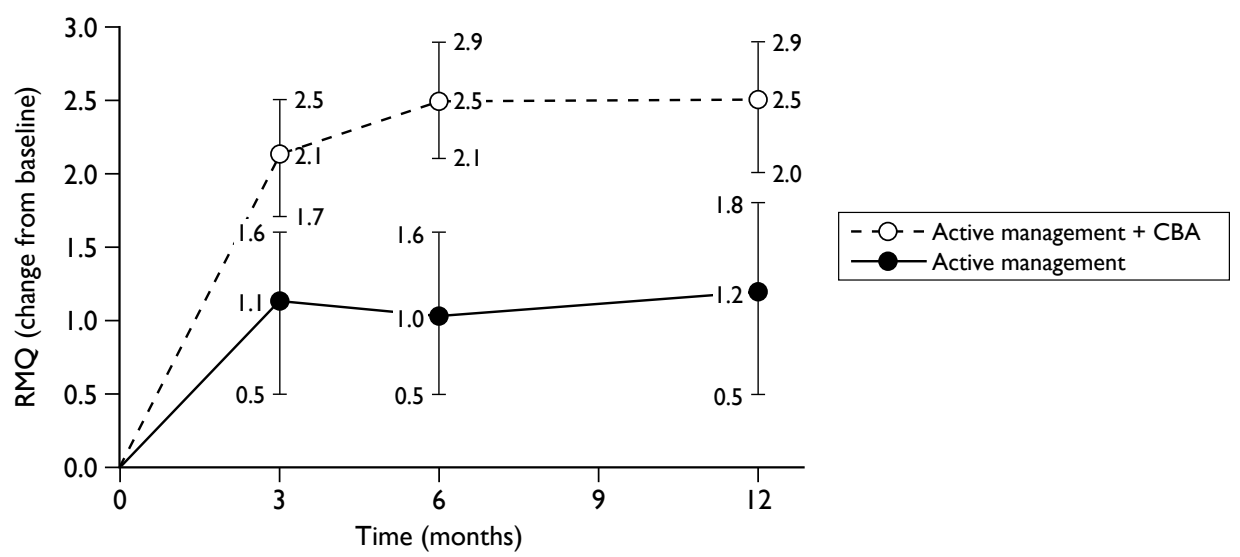

FIGURE 8 Roland Morris Questionnaire (RMQ) scores (change from baseline). Least square estimates of the mean ( $95 \% \mathrm{Cl})$ from the linear regression models (observed data).

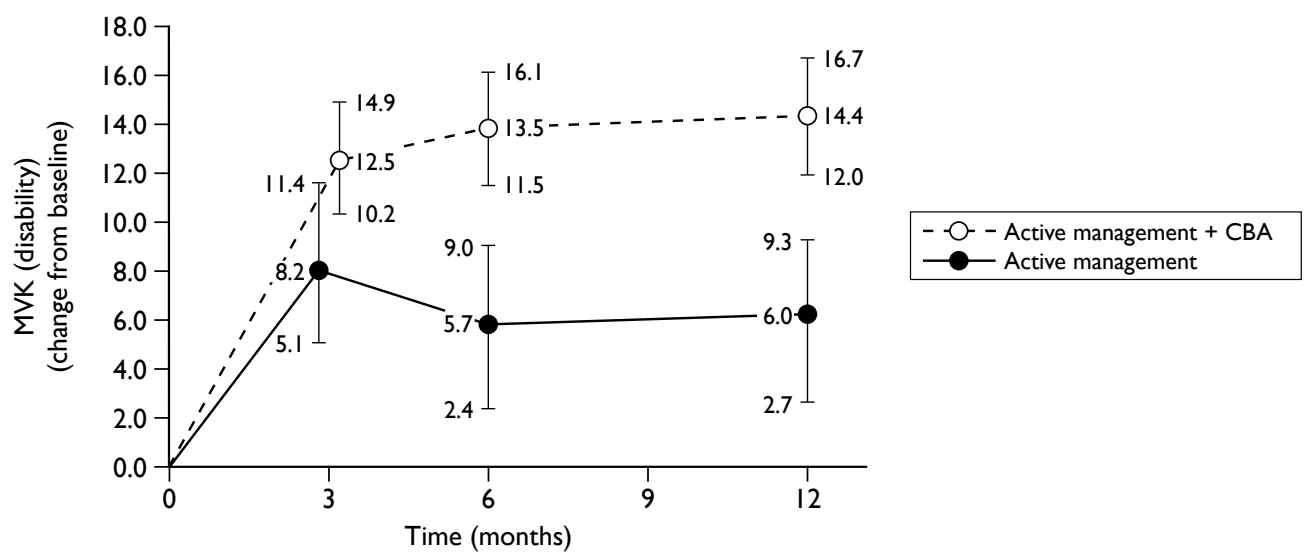

FIGURE 9 Modified Von Korff (MVK) (disability) scores (change from baseline). Least square estimates of the mean (95\% Cl) from the linear regression models (observed data).

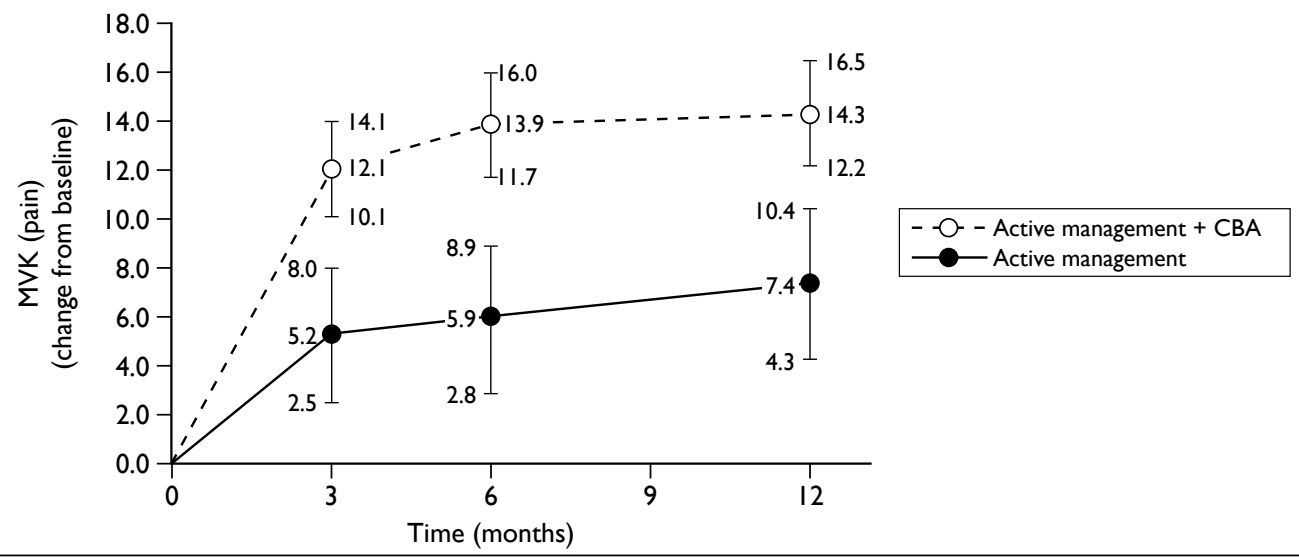

FIGURE 10 Modified Von Korff (MVK) (pain) scores (change from baseline). Least square estimates of the mean (95\% Cl) from the linear regression models (observed data). 
Improvements in the CBA arm were larger, with the greatest improvement at 12 months.

The difference between CBA and AM was estimated to be on average 2.2 at 3 months, 1.8 at 6 months and 4.1 at 12 months. The differences between treatments were statistically significant at all time points.

\section{Short Form-12 mental subscale}

Figure 12 shows the change in SF-12 mental subscale by treatment arm. In the AM arm, there was no change in mental-health score across the 12 months. In the CBA arm, there were statistically significant improvements in mental health at 6 months, with the maximum improvement at 6 months. By 12 months, mental-health scores fell, and there was no statistically significant difference between the CBA and AM arms.
The difference between CBA and AM was estimated to be on average 1.3 at 3 months, 2.5 at 6 months, and 0.1 at 12 months.

\section{Fear Avoidance Beliefs Questionnaire}

Figure 13 shows changes in fear avoidance beliefs by treatment arm. In the AM arm, there was no evidence of change in fear avoidance beliefs across the 12 months. In the CBA arm, there were substantial improvements in fear avoidance at 3, 6 and 12 months. Improvement occurred between 0 and 3 months and was sustained to 12 months.

The difference between CBA and AM was estimated to be on average 2.6 at 3 months, 3.1 at 6 months and 3.0 at 12 months. The differences between treatments were statistically significant at all time points.

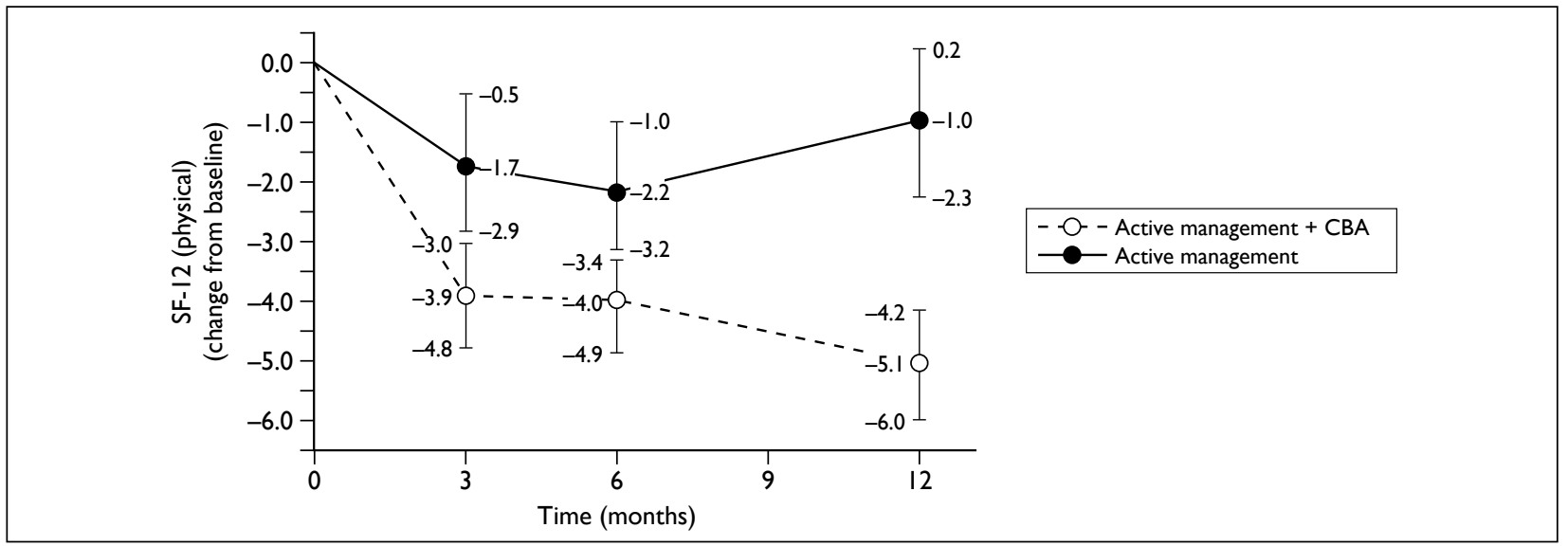

FIGURE II Short Form 12-item health survey (physical) scores (change from baseline). Least square estimates of the mean (95\% CI) from the linear regression models (observed data).

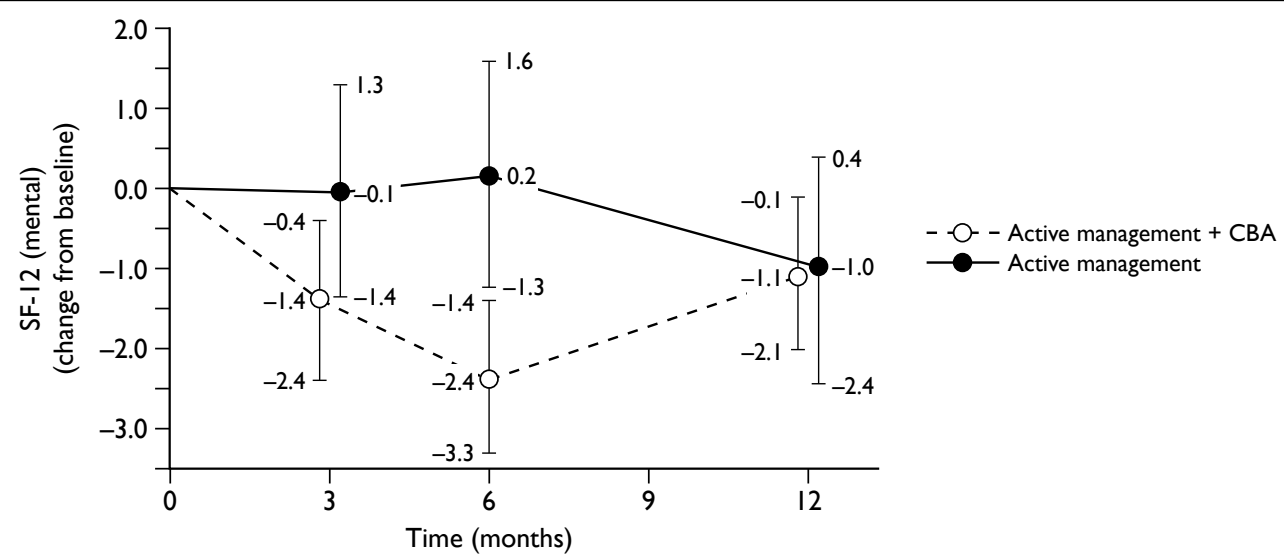

FIGURE I2 Short Form 12-item health survey (mental) scores (change from baseline). Least square estimates of the mean (95\% Cl) from the linear regression models (observed data). 


\section{Pain self-efficacy}

Figure 14 shows pain self-efficacy by treatment arm. There was no discernible change in pain self-efficacy in the AM arm. Self-efficacy improved in the CBA arm, with maximal improvements at 6 months, and sustained improvement at 12 months.

The difference between CBA and AM was estimated to be on average 3.2 at 3 months, 4.1 at 6 months and 3.8 at 12 months. The differences between treatment arms were statistically significant at all time points.

\section{Changes in employment and hours worked}

The number and proportion of participants who were receiving any entitlements/benefits were very small for both therapy groups. There was a small difference in the numbers of people changing their hours of work between the groups (in favour of the CBA) but these differences were not statistically significant. A slightly higher proportion of people randomised to CBA reported having sick days at 3 and 6 months, but this difference was small and not statistically significant. Overall there were no differences in the numbers of days off sick. Data on sickness, benefit claim and employment are given in Appendix 13.

\section{Global indicators of change, benefit and satisfaction with treatment}

Self-rated global assessments of change in back pain and satisfaction with treatment favoured CBA over AM (data shown in Table 27). Differences were statistically significant for all comparisons regardless of the time point of follow-up.

By 12 months, $60 \%$ of participants in the CBA arm rated that they had improvements, whereas

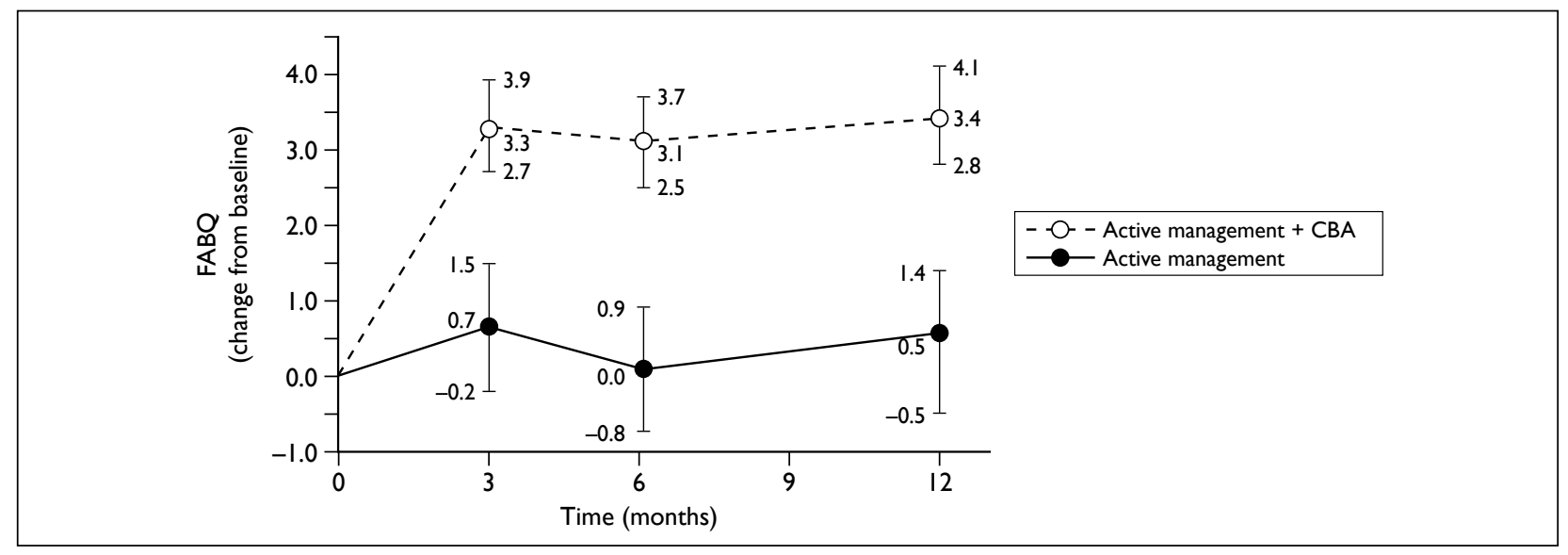

FIGURE I3 Fear Avoidance Beliefs Questionnaire (FABQ) (physical activity) scores (change from baseline). Least square estimates of the mean $(95 \% \mathrm{Cl})$ from the linear regression models (observed data).

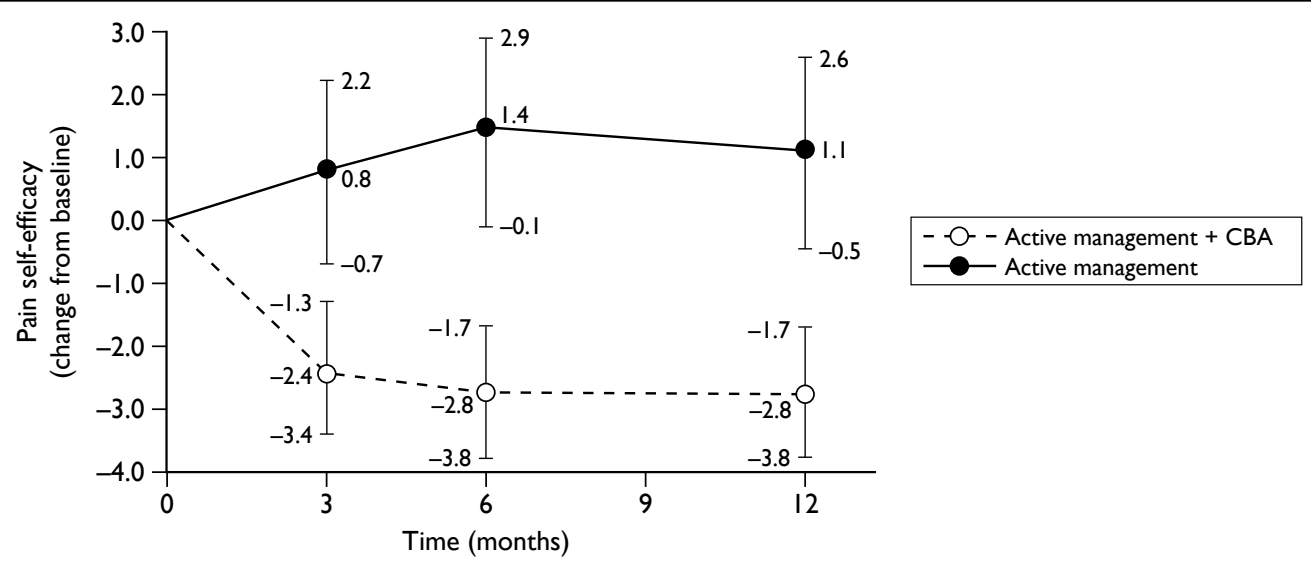

FIGURE I4 Pain Self-efficacy scores (change from baseline). Least square estimates of the mean (95\% Cl) from the linear regression models (observed data). 
TABLE 27 Change in back pain and treatment satisfaction (n, \%)

\begin{tabular}{|c|c|c|c|c|}
\hline Month & & AM & AM+CBA & $p$-value \\
\hline \multicolumn{5}{|c|}{ Change in back pain } \\
\hline \multirow[t]{7}{*}{3} & Completely recovered & $\mathrm{I}(0.5)$ & $3(I)$ & $<0.0001$ \\
\hline & Much improved & $8(4)$ & $95(27)$ & \\
\hline & Slightly improved & $45(24)$ & $98(28)$ & \\
\hline & No change & $93(50)$ & $124(35)$ & \\
\hline & Slightly worsened & $29(16)$ & $20(6)$ & \\
\hline & Much worsened & $8(4)$ & $12(3)$ & \\
\hline & Vastly worsened & $3(2)$ & $2(I)$ & \\
\hline \multirow[t]{7}{*}{6} & Completely recovered & $2(I)$ & $31(12)$ & $<0.0001$ \\
\hline & Much improved & $19(10)$ & $105(4 I)$ & \\
\hline & Slightly improved & $35(19)$ & $82(32)$ & \\
\hline & No change & 81 (44) & $133(11)$ & \\
\hline & Slightly worsened & $27(15)$ & $14(I)$ & \\
\hline & Much worsened & $18(10)$ & $20(2)$ & \\
\hline & Vastly worsened & $4(2)$ & $\mathrm{I}(\mathrm{I})$ & \\
\hline \multirow[t]{7}{*}{12} & Completely recovered & $6(3)$ & $44(\mathrm{II})$ & $<0.0001$ \\
\hline & Much improved & $24(12)$ & $106(27)$ & \\
\hline & Slightly improved & $32(16)$ & $85(22)$ & \\
\hline & No change & $89(45)$ & $122(3 \mid)$ & \\
\hline & Slightly worsened & $24(12)$ & $20(5)$ & \\
\hline & Much worsened & $17(9)$ & $9(2)$ & \\
\hline & Vastly worsened & $5(3)$ & $9(2)$ & \\
\hline \multicolumn{5}{|c|}{ Satisfaction } \\
\hline \multirow[t]{5}{*}{3} & Very dissatisfied & $19(11)$ & $20(6)$ & $<0.0001$ \\
\hline & Somewhat dissatisfied & $23(13)$ & II (3) & \\
\hline & Neither satisfied nor dissatisfied & $75(44)$ & $46(14)$ & \\
\hline & Somewhat satisfied & $38(22)$ & $117(35)$ & \\
\hline & Very satisfied & $17(10)$ & $137(4 \mid)$ & \\
\hline \multirow[t]{5}{*}{6} & Very dissatisfied & $20(12)$ & $28(8)$ & $<0.0001$ \\
\hline & Somewhat dissatisfied & $20(12)$ & $23(9)$ & \\
\hline & Neither satisfied nor dissatisfied & $84(52)$ & 7I (20) & \\
\hline & Somewhat satisfied & $24(15)$ & III (3I) & \\
\hline & Very satisfied & $15(9)$ & $110(3 \mathrm{I})$ & \\
\hline \multirow[t]{5}{*}{12} & Very dissatisfied & $14(9)$ & $19(6)$ & $<0.0001$ \\
\hline & Somewhat dissatisfied & $22(15)$ & $21(6)$ & \\
\hline & Neither satisfied nor dissatisfied & $72(48)$ & $76(23)$ & \\
\hline & Somewhat satisfied & $30(20)$ & $104(32)$ & \\
\hline & Very satisfied & $13(9)$ & $108(33)$ & \\
\hline
\end{tabular}


$31 \%$ in the AM arm reported improvement. Less than $25 \%$ of people randomised to AM reported a decline at 12 months, in comparison with $9 \%$ in the CBA arm. Similar trends were noted in the benefit question. Levels of satisfaction were greater in the CBA arm in comparison with AM.

\section{Numbers needed to treat}

Using a threshold of change of $30 \%$ to determine an MCID approximately seven participants would be needed on the AM+CBA arm to see one participant improve. The same estimate was gained using the MVK scales.

The numbers needed to treat using the global indicator of change scale was 7 (improvement versus stability or decline) or 3 (improvement and stability versus decline).

\section{Estimates of the standardised difference (effect size)}

The effect sizes for each of the outcomes are derived from the adjusted treatment estimates (Table 28).

\section{Adverse events}

Only one adverse event occurred during the study, and this involved a participant receiving AM. An adverse event notification (Appendix 10) was submitted to West Midlands MREC on 3 August 2006. This patient was admitted to hospital on 11 July 2006 with acute spinal cord compression, which was subsequently decompressed operatively. Following discharge, the participant developed a pulmonary embolus as a complication, which

TABLE 28 Effect sizes of the clinical outcome measures at 12 months based on unadjusted differences estimated in regression modelling

\begin{tabular}{ll} 
& Effect size \\
\hline RMQ (absolute) & 0.31 \\
MVK (disability) & 0.41 \\
MVK (pain) & 0.37 \\
Pain self-efficacy & 0.35 \\
FABQ & 0.47 \\
SF-12 (physical) & 0.45 \\
SF-12 (mental) & 0.07 \\
FABQ, Fear Avoidance Beliefs Questionnaire; MVK, \\
Modified Von Korff Scale; RMQ, Roland Morris \\
Disability Questionnaire; SF-12, Short Form I2-item \\
health survey.
\end{tabular}

required readmission. Final discharge was on 27 July 2006. As part of the 'control intervention', the patient received The Back Book and a single session of advice. There is no indication that advice to stay active increases the risk of spinal cord compression, which affects a small proportion of back pain patients. The Back Book gives details of warning signs and advises immediate consultation with a doctor. The patient followed this advice and therefore the emergency was dealt with appropriately without delay.

\section{Subgroup analysis}

We undertook three prespecified subgroup analyses and one additional subgroup analysis based on the RMQ baseline score. For each of the three subgroups, no statistical interactions were found between the subgroups and the treatment assigned.

\section{Fear avoidance beliefs at baseline}

Results are given in Table 29. Fear avoidance at baseline was not associated with the size of treatment effect measured using the RMQ or the MVK (pain) either at 12 months or when considered over the entire follow-up period. However, there was a statistically significant interaction between baseline fear avoidance and outcomes measured using the MVK for disability. The treatment effect was substantially larger in the cohort who had baseline fear avoidance scores $<14$ (i.e. were not fear avoidance at baseline). However, these observations were not consistent across all primary outcomes.

\section{Pain severity (troublesomeness) at baseline}

Results are given in Table 30. Estimates of the treatment effect were larger in people with moderately troublesome LBP as opposed to severe LBP. Active management had little or no effect in those with moderately troublesome back pain, which accounted for this. The interaction term was not statistically significant $(p=0.07)$. When the effect over the whole year was considered there was no indication of a difference between the two groups.

\section{Duration of back pain}

Results are given in Table 31. Estimates of the treatment effect were larger in people with 
TABLE 29 Subgroup analysis - linear regression and longitudinal models assessing the RMQ, MVK (disability) and MVK (pain) changes from baseline to 12 months and overall change from baseline respectively, for those with fear avoidance of $<14$ and $\geq 14$ at baseline

\begin{tabular}{|c|c|c|c|c|c|c|c|}
\hline & & & Mean & SE & $\mathbf{N}$ & $\begin{array}{l}\text { Treatment } \\
\text { estimate }(95 \% \mathrm{Cl})\end{array}$ & $\begin{array}{l}\text { p-value } \\
\text { (interaction) }\end{array}$ \\
\hline \multicolumn{8}{|c|}{ Roland Morris Disability Questionnaire } \\
\hline \multicolumn{8}{|c|}{12 months } \\
\hline \multirow{4}{*}{$\begin{array}{l}\text { Fear avoidance } \\
\times \text { treatment }\end{array}$} & $<14$ & AM & 1.1 & 0.49 & 72 & & 0.56 \\
\hline & $<14$ & $\mathrm{AM}+\mathrm{CBA}$ & 2.5 & 0.33 & 156 & $\mathrm{I} .3(0.19$ to $2.5 \mathrm{I})$ & \\
\hline & $\geq 14$ & AM & 0.9 & 0.47 & 77 & & \\
\hline & $\geq 14$ & $\mathrm{AM}+\mathrm{CBA}$ & 2.7 & 0.32 & 165 & I.8 (0.7I to 2.95$)$ & \\
\hline \multicolumn{8}{|l|}{ Longitudinal } \\
\hline \multirow{4}{*}{$\begin{array}{l}\text { Fear avoidance } \\
\times \text { treatment }\end{array}$} & $<14$ & AM & 0.8 & 0.39 & 223 & & 0.74 \\
\hline & $<14$ & $\mathrm{AM}+\mathrm{CBA}$ & 2.3 & 0.27 & 463 & $1.5(0.58$ to 2.46$)$ & \\
\hline & $\geq 14$ & AM & 1.1 & 0.33 & 261 & & \\
\hline & $\geq 14$ & $\mathrm{AM}+\mathrm{CBA}$ & 2.4 & 0.25 & 511 & $1.3(0.47$ to 2.15$)$ & \\
\hline \multicolumn{8}{|c|}{ Modified Von Korff (disability) } \\
\hline \multicolumn{8}{|c|}{12 months } \\
\hline \multirow{4}{*}{$\begin{array}{l}\text { Fear avoidance } \\
\times \text { treatment }\end{array}$} & $<14$ & AM & 1.6 & 2.97 & 77 & 14.8 (7.76 to 21.88$)$ & 0.07 \\
\hline & $<14$ & $\mathrm{AM}+\mathrm{CBA}$ & 16.4 & 2.03 & 165 & & \\
\hline & $\geq 14$ & AM & 8.2 & 2.62 & 99 & $6.0(-0.36$ to 12.38$)$ & \\
\hline & $\geq 14$ & $\mathrm{AM}+\mathrm{CBA}$ & 14.2 & 1.93 & 182 & & \\
\hline \multicolumn{8}{|l|}{ Longitudinal } \\
\hline \multirow{4}{*}{$\begin{array}{l}\text { Fear avoidance } \\
x \text { treatment }\end{array}$} & $<14$ & AM & 3.1 & 2.34 & 228 & 11.7 (6.09 to 17.23) & 0.03 \\
\hline & $<14$ & $\mathrm{AM}+\mathrm{CBA}$ & 14.7 & 1.61 & 480 & & \\
\hline & $\geq 14$ & AM & 8.5 & 2.04 & 292 & $4.8(-0.19$ to 9.77$)$ & \\
\hline & $\geq 14$ & $\mathrm{AM}+\mathrm{CBA}$ & 13.3 & 1.52 & 540 & & \\
\hline \multicolumn{8}{|c|}{ Modified Von Korff (pain) } \\
\hline \multicolumn{8}{|c|}{12 months } \\
\hline \multirow{4}{*}{$\begin{array}{l}\text { Fear avoidance } \\
\times \text { treatment }\end{array}$} & $<14$ & AM & 9.4 & 2.52 & 80 & $6.6(0.66$ to 12.58$)$ & 0.92 \\
\hline & $<14$ & $\mathrm{AM}+\mathrm{CBA}$ & 16.0 & 1.69 & 179 & & \\
\hline & $\geq 14$ & AM & 6.5 & 2.23 & 103 & 7.1 (1.62 to 12.48$)$ & \\
\hline & $\geq 14$ & $\mathrm{AM}+\mathrm{CBA}$ & 13.5 & 1.64 & 189 & & \\
\hline \multicolumn{8}{|l|}{ Longitudinal } \\
\hline \multirow{4}{*}{$\begin{array}{l}\text { Fear avoidance } \\
\times \text { treatment }\end{array}$} & $<14$ & AM & 7.3 & 1.96 & 235 & 6.9 (2.03 to II.33) & 0.91 \\
\hline & $<14$ & $\mathrm{AM}+\mathrm{CBA}$ & 14.0 & 1.34 & 504 & & \\
\hline & $\geq 14$ & AM & 5.6 & 1.71 & 299 & 7.1 (2.90 to II.22) & \\
\hline & $\geq 14$ & $\mathrm{AM}+\mathrm{CBA}$ & 12.7 & 1.26 & 562 & & \\
\hline
\end{tabular}

moderately troublesome low back pain as opposed to severe low back pain. Active management having little or no effect in those with moderately troublesome back pain accounted for this. The interaction term was not found to be statistically significant $(p=0.07)$. When the effect over the whole year was considered there was no indication of a difference between the two groups. 
TABLE 30 Subgroup analysis - linear regression and longitudinal models assessing the RMQ, MVK (disability) and MVK (pain) change from baseline to 12 months and overall change from baseline respectively, for those with pain severity as moderate and very/extremely troublesome

\begin{tabular}{|c|c|c|c|c|c|c|c|}
\hline & & & Mean & SE & $\mathbf{N}$ & $\begin{array}{l}\text { Treatment } \\
\text { estimate }(95 \% \mathrm{Cl})\end{array}$ & $\begin{array}{l}p \text {-value } \\
\text { (interaction) }\end{array}$ \\
\hline \multicolumn{8}{|c|}{ Roland Morris Disability Questionnaire } \\
\hline \multicolumn{8}{|c|}{12 months } \\
\hline \multirow{4}{*}{$\begin{array}{l}\text { Pain severity } \\
\times \text { Treatment }\end{array}$} & Moderate & AM & 0.3 & 0.42 & 96 & 2.1 (1.07 to 3.07$)$ & 0.07 \\
\hline & Moderate & $\mathrm{AM}+\mathrm{CBA}$ & 2.4 & 0.30 & 185 & & \\
\hline & Severe/very severe & AM & 2.1 & 0.52 & 63 & $-0.6(-1.82$ to 0.58$)$ & \\
\hline & Severe/very severe & $\mathrm{AM}+\mathrm{CBA}$ & 2.7 & 0.33 & 154 & & \\
\hline \multicolumn{8}{|l|}{ Longitudinal } \\
\hline \multirow{4}{*}{$\begin{array}{l}\text { Pain severity } \\
\times \text { treatment }\end{array}$} & Moderate & AM & 0.4 & 0.34 & 297 & $\mathrm{I} .7(0.90$ to $2.5 \mathrm{I})$ & 0.12 \\
\hline & Moderate & $\mathrm{AM}+\mathrm{CBA}$ & 2.1 & 0.24 & 557 & & \\
\hline & Severe/very severe & AM & 1.9 & 0.38 & 216 & $0.7(-0.19$ to $1.6 \mathrm{I})$ & \\
\hline & Severe/very severe & $\mathrm{AM}+\mathrm{CBA}$ & 2.6 & 0.26 & 469 & & \\
\hline \multicolumn{8}{|c|}{ Modified Von Korff (disability) } \\
\hline \multicolumn{8}{|c|}{12 months } \\
\hline \multirow{4}{*}{$\begin{array}{l}\text { Pain severity } \\
\times \text { treatment }\end{array}$} & Moderate & AM & 2.6 & 2.50 & 107 & II.6 (5.53 to 17.73) & 0.33 \\
\hline & Moderate & $\mathrm{AM}+\mathrm{CBA}$ & 14.3 & 1.85 & 197 & & \\
\hline & Severe/very severe & AM & 8.7 & 2.91 & 79 & 7.1 (0.1I to 13.97) & \\
\hline & Severe/very severe & $\mathrm{AM}+\mathrm{CBA}$ & 15.8 & 1.99 & 169 & & \\
\hline \multicolumn{8}{|l|}{ Longitudinal } \\
\hline \multirow{4}{*}{$\begin{array}{l}\text { Pain severity } \\
\times \text { treatment }\end{array}$} & Moderate & AM & 3.5 & 1.99 & 308 & 8.6 (3.78 to 13.42$)$ & 0.49 \\
\hline & Moderate & $\mathrm{AM}+\mathrm{CBA}$ & 12.1 & 1.46 & 574 & & \\
\hline & Severe/very severe & AM & 9.6 & 2.23 & 242 & 6.1 (0.76 to II.42) & \\
\hline & Severe/very severe & $\mathrm{AM}+\mathrm{CBA}$ & 15.7 & 1.56 & 498 & & \\
\hline \multicolumn{8}{|c|}{ Modified Von Korff (pain) } \\
\hline \multicolumn{8}{|l|}{12 months } \\
\hline \multirow{4}{*}{$\begin{array}{l}\text { Pain severity } \\
\times \text { treatment }\end{array}$} & Moderate & $A M$ & 3.9 & 2.14 & 110 & $9.0(3.83$ to 14.17$)$ & 0.27 \\
\hline & Moderate & $\mathrm{AM}+\mathrm{CBA}$ & 12.9 & 1.55 & 209 & & \\
\hline & Severe/very severe & AM & 11.2 & 2.45 & 84 & $4.6(-1.16$ to 10.44$)$ & \\
\hline & Severe/very severe & $\mathrm{AM}+\mathrm{CBA}$ & 15.9 & 1.67 & 180 & & \\
\hline \multicolumn{8}{|l|}{ Longitudinal } \\
\hline \multirow{4}{*}{$\begin{array}{l}\text { Pain severity } \\
\times \text { treatment }\end{array}$} & Moderate & AM & 3.9 & 1.66 & 316 & 7.0 (3.02 to 11.02$)$ & 0.87 \\
\hline & Moderate & $A M+C B A$ & 10.9 & 1.20 & 602 & & \\
\hline & Severe/very severe & AM & 8.8 & 1.85 & 250 & 6.5 (2.10 to 10.92$)$ & \\
\hline & Severe/very severe & $\mathrm{AM}+\mathrm{CBA}$ & 15.3 & 1.29 & 522 & & \\
\hline
\end{tabular}


TABLE 3 I Subgroup analysis - linear regression and longitudinal models assessing the RMQ, MVK (disability) and MVK (pain) change from baseline to 12 months and overall change from baseline respectively, for those with duration of back pain as recorded at baseline of $\leq 3$ years and $>3$ years

\begin{tabular}{|c|c|c|c|c|c|c|c|}
\hline & & & Mean & SE & $\mathbf{N}$ & $\begin{array}{l}\text { Treatment estimate } \\
(95 \% \mathrm{CI})\end{array}$ & $\begin{array}{l}p \text {-value } \\
\text { (interaction) }\end{array}$ \\
\hline \multicolumn{8}{|c|}{ Roland Morris Disability Questionnaire } \\
\hline \multicolumn{8}{|l|}{12 months } \\
\hline \multirow{4}{*}{$\begin{array}{l}\text { Duration (years) } \\
\times \text { treatment }\end{array}$} & $\leq 3$ & AM & 1.8 & 0.62 & 40 & $1.5(-0.08$ to 3.02$)$ & 0.96 \\
\hline & $\leq 3$ & $\mathrm{AM}+\mathrm{CBA}$ & 3.2 & 0.45 & 83 & & \\
\hline & $>3$ & AM & 0.8 & 0.39 & 115 & 1.5 (0.59 to 2.43$)$ & \\
\hline & $>3$ & $\mathrm{AM}+\mathrm{CBA}$ & 2.3 & 0.27 & 240 & & \\
\hline \multicolumn{8}{|l|}{ Longitudinal } \\
\hline \multirow{4}{*}{$\begin{array}{l}\text { Duration (years) } \\
\times \text { treatment }\end{array}$} & $\leq 3$ & AM & 1.3 & 0.50 & $|3|$ & $1.5(0.33$ to 2.73$)$ & 0.60 \\
\hline & $\leq 3$ & $\mathrm{AM}+\mathrm{CBA}$ & 2.9 & 0.36 & 262 & & \\
\hline & $>3$ & AM & 0.9 & 0.30 & 369 & I.2 (0.43 to I.89) & \\
\hline & $>3$ & $\mathrm{AM}+\mathrm{CBA}$ & 2.1 & 0.22 & 713 & & \\
\hline \multicolumn{8}{|c|}{ Modified Von Korff (disability) } \\
\hline \multicolumn{8}{|c|}{12 months } \\
\hline \multirow{4}{*}{$\begin{array}{l}\text { Duration (years) } \\
\times \text { treatment }\end{array}$} & $\leq 3$ & AM & 8.2 & 3.61 & 51 & 13.9 (5.17 to 22.57$)$ & 0.33 \\
\hline & $\leq 3$ & $\mathrm{AM}+\mathrm{CBA}$ & 22.1 & 2.60 & 98 & & \\
\hline & $>3$ & AM & 4.1 & 2.25 & $|3|$ & 8.7 (3.29 to 14.15$)$ & \\
\hline & $>3$ & $\mathrm{AM}+\mathrm{CBA}$ & 12.8 & 1.62 & 251 & & \\
\hline \multicolumn{8}{|l|}{ Longitudinal } \\
\hline \multirow{4}{*}{$\begin{array}{l}\text { Duration (years) } \\
\times \text { treatment }\end{array}$} & $\leq 3$ & AM & 9.1 & 2.82 & 153 & I0.I (5.23 to 14.99) & 0.49 \\
\hline & $\leq 3$ & $\mathrm{AM}+\mathrm{CBA}$ & 19.2 & 2.06 & 285 & & \\
\hline & $>3$ & AM & 5.1 & 1.76 & 386 & 7.3 (3.04 to II.54) & \\
\hline & $>3$ & $\mathrm{AM}+\mathrm{CBA}$ & 12.4 & 1.29 & 735 & & \\
\hline \multicolumn{8}{|c|}{ Modified Von Korff (pain) } \\
\hline \multicolumn{8}{|l|}{12 months } \\
\hline \multirow{4}{*}{$\begin{array}{l}\text { Duration (years) } \\
\times \text { treatment }\end{array}$} & $\leq 3$ & AM & 7.9 & 3.17 & 51 & $10.9(3.30$ to 18.50$)$ & 0.26 \\
\hline & $\leq 3$ & $\mathrm{AM}+\mathrm{CBA}$ & 18.8 & 2.22 & 104 & & \\
\hline & $>3$ & $A M$ & 7.2 & 1.93 & 138 & $5.8(1.12$ to 10.44$)$ & \\
\hline & $>3$ & $\mathrm{AM}+\mathrm{CBA}$ & 13.0 & 1.39 & 267 & & \\
\hline \multicolumn{8}{|l|}{ Longitudinal } \\
\hline \multirow{4}{*}{$\begin{array}{l}\text { Duration (years) } \\
\times \text { treatment }\end{array}$} & $\leq 3$ & $\mathrm{AM}$ & 7.6 & 2.38 & 153 & $8.2(2.50$ to 13.94$)$ & 0.65 \\
\hline & $\leq 3$ & $A M+C B A$ & 15.8 & 1.70 & 303 & & \\
\hline & $>3$ & AM & 5.8 & $\mathrm{I} .47$ & 399 & 6.7 (3.10 to 10.20$)$ & \\
\hline & $>3$ & $\mathrm{AM}+\mathrm{CBA}$ & 12.5 & 1.07 & 769 & & \\
\hline
\end{tabular}


TABLE 32 Subgroup analysis - linear regression and longitudinal models assessing the RMQ, MVK (disability) and MVK (pain) change from baseline to 12 months and overall change from baseline respectively, for those with $R M Q<4$ and $\geq 4$ at baseline

\begin{tabular}{|c|c|c|c|c|c|c|c|}
\hline & & & Mean & SE & $\mathbf{N}$ & $\begin{array}{l}\text { Treatment estimate } \\
(95 \% \mathrm{Cl})\end{array}$ & $\begin{array}{l}p \text {-value } \\
\text { (interaction) }\end{array}$ \\
\hline \multicolumn{8}{|c|}{ Roland Morris Disability Questionnaire } \\
\hline \multirow{4}{*}{$\begin{array}{l}\text { Baseline RMQ } \\
\times \text { treatment }\end{array}$} & $<4$ & AM & -0.2 & 0.75 & 29 & & 0.20 \\
\hline & $<4$ & $\mathrm{AM}+\mathrm{CBA}$ & 0.2 & 0.53 & 57 & $0.4(-1.37$ to 2.23$)$ & \\
\hline & $\geq 4$ & AM & 1.3 & 0.35 & 130 & & \\
\hline & $\geq 4$ & $\mathrm{AM}+\mathrm{CBA}$ & 3.0 & 0.24 & 282 & $1.7(0.88$ to 2.56$)$ & \\
\hline \multicolumn{8}{|l|}{ Longitudinal } \\
\hline \multirow{4}{*}{$\begin{array}{l}\text { Baseline RMQ } \\
\times \text { treatment }\end{array}$} & $<4$ & AM & -0.3 & 0.61 & 88 & & 0.08 \\
\hline & $<4$ & $A M+C B A$ & -0.2 & 0.43 & 138 & $0.1(-1.37$ to 1.57$)$ & \\
\hline & $\geq 4$ & AM & 1.3 & 0.27 & 425 & & \\
\hline & $\geq 4$ & $\mathrm{AM}+\mathrm{CBA}$ & 2.8 & 0.19 & 858 & $1.5(0.86$ to 2.16$)$ & \\
\hline \multicolumn{8}{|c|}{ Modified Von Korff (disability) } \\
\hline \multicolumn{8}{|c|}{12 months } \\
\hline \multirow{4}{*}{$\begin{array}{l}\text { Baseline RMQ } \\
\times \text { treatment }\end{array}$} & $<4$ & AM & 0.9 & 4.58 & 32 & $9.4(-1.64$ to 20.40$)$ & 0.94 \\
\hline & $<4$ & $\mathrm{AM}+\mathrm{CBA}$ & 10.3 & 3.26 & 63 & & \\
\hline & $\geq 4$ & AM & 6.1 & 2.09 & 154 & 9.8 (4.80 to 14.84$)$ & \\
\hline & $\geq 4$ & $\mathrm{AM}+\mathrm{CBA}$ & 15.9 & 1.49 & 303 & & \\
\hline \multicolumn{8}{|l|}{ Longitudinal } \\
\hline \multirow{4}{*}{$\begin{array}{l}\text { Baseline RMQ } \\
\text { × treatment }\end{array}$} & $<4$ & AM & 3.1 & 3.79 & 88 & $6.2(2.81$ to 15.15$)$ & 0.72 \\
\hline & $<4$ & $\mathrm{AM}+\mathrm{CBA}$ & 9.3 & 2.57 & 185 & & \\
\hline & $\geq 4$ & AM & 6.8 & 1.62 & 462 & 7.9 (4.02 to II.86) & \\
\hline & $\geq 4$ & $\mathrm{AM}+\mathrm{CBA}$ & 14.7 & 1.18 & 887 & & \\
\hline \multicolumn{8}{|c|}{ Modified Von Korff (pain) } \\
\hline \multicolumn{8}{|c|}{12 months } \\
\hline \multirow{4}{*}{$\begin{array}{l}\text { Baseline RMQ } \\
\times \text { treatment }\end{array}$} & $<4$ & AM & 8.7 & 3.92 & 33 & $2.8(-6.61$ to 12.18$)$ & 0.31 \\
\hline & $<4$ & $\mathrm{AM}+\mathrm{CBA}$ & 11.5 & 2.73 & 68 & & \\
\hline & $\geq 4$ & AM & 6.8 & 1.78 & 161 & 8.1 (3.85 to 12.39) & \\
\hline & $\geq 4$ & $\mathrm{AM}+\mathrm{CBA}$ & 14.9 & 1.26 & 321 & & \\
\hline \multicolumn{8}{|l|}{ Longitudinal } \\
\hline \multirow{4}{*}{$\begin{array}{l}\text { Baseline RMQ } \\
\times \text { treatment }\end{array}$} & $<4$ & $\mathrm{AM}$ & 7.7 & 3.13 & 92 & $2.0(-5.46$ to 9.40$)$ & 0.16 \\
\hline & $<4$ & $\mathrm{AM}+\mathrm{CBA}$ & 9.7 & 2.15 & 191 & & \\
\hline & $\geq 4$ & $A M$ & 5.8 & 1.35 & 474 & 7.9 (4.60 to II.I0) & \\
\hline & $\geq 4$ & $A M+C B A$ & 13.6 & 0.97 & 933 & & \\
\hline
\end{tabular}




\section{Roland Morris score}

Results are given in Table 32. The trend was toward large treatment effects in those participants with an RMQ greater than 4 at baseline. However, the interaction tests were not statistically significant. There was no evidence of a difference in models where the outcome was the MVK.

\section{Sensitivity analysis}

\section{Per protocol analysis}

There was no difference in the results when they were analysed using only those individuals who had attended at least the assessment and three sessions of CBA.

\section{Multiple imputation of missing data}

There was no difference in the results between the observed cases analysis and an analysis based on multiple imputations. The findings appear insensitive to the method of dealing with missing data. 



\section{Chapter 5 \\ Qualitative study}

\section{BeST interview study}

\section{Introduction}

The aims of the interview study were to explore user perspectives on the acceptability of both interventions tested and to gain insight into how the interventions might work. Previous studies have indicated the importance of understanding the patient's perspective within research on LBP. ${ }^{127-130}$

\section{Method}

We performed 34 semi-structured interviews, 4-5 months after randomisation, and after participants had received their intervention. Eighteen participants were from the AM arm and 16 were from the $\mathrm{AM}+\mathrm{CBA}$ arm of the main BeST trial.

\section{Sample}

The interviewees were sampled purposively from two clusters local to the trial coordinating centre for pragmatic reasons of time and cost [Coventry and Solihull (one cluster) and South Warwickshire]. Sampling aimed for equal numbers of interviewees from the intervention and control arm of the study and a range of two key participant characteristics which relate to the nature of the intervention: disability as measured by the RMQ and fear avoidance as measured by the FABQ. Sampling categories for disability and fear avoidance were developed as follows. The RMQ has a 24-point scale and the median of the first 100 baseline scores was 9 . Two categories were used for sampling: high (9-24) and low (0-8). As the CBA is aimed at reducing fear avoidance we also selected the interviewees across three bands of the FABQ to ensure diversity; 0-9 for 'non-avoiders', 18-24 for 'avoiders' and a mid-range group of 10-17.

We aimed for equal numbers of interviewees in each of the cells of Table 33. However, the category representing high $R M Q$ and low $F A B Q$ scores had few potential candidates.

Where there was a choice of potential interviewees we aimed to select individuals to reflect key demographic characteristics of the sample included in the clinical trial. We modelled this on the characteristics of the first 100 participants, and considered self-rated pain severity (as given by participants at the randomisation appointment) and gender representation in particular.

\section{Interviewee recruitment}

Participants were eligible to be contacted for interview if they had agreed to this on their initial consent form. A total of 40 of the $701 \mathrm{BeST}$ trial participants were invited for interview by a phone call from a researcher (V.N.) who outlined that the face-to-face interview would last approximately 45 minutes and be held at a venue of their choice (Figure 15). Potential participants were informed that the interviews would be about their experience of back pain and their views of the interventions tested in the trial, and were briefed that the interviews would be recorded if they were in agreement. Four interviews were undertaken as a pilot and are not included in the final analysis. Two potential participants declined to be interviewed: one because of an operation; the other did not give a reason.

TABLE 33 Number of interviewees in each sample category

\begin{tabular}{|llll|} 
& $\begin{array}{l}\text { Avoiders } \\
\text { (FABQ= 18-24) }\end{array}$ & $\begin{array}{l}\text { Mixed } \\
(\text { FABQ = 10-17) }\end{array}$ & $\begin{array}{l}\text { Non-avoiders } \\
\text { (FABQ=0-9) }\end{array}$ \\
\hline High disability (RMQ=9-24) & 6 & 7 & 3 \\
Low disability (RMQ=0-8) & 5 & 7 & 6
\end{tabular}




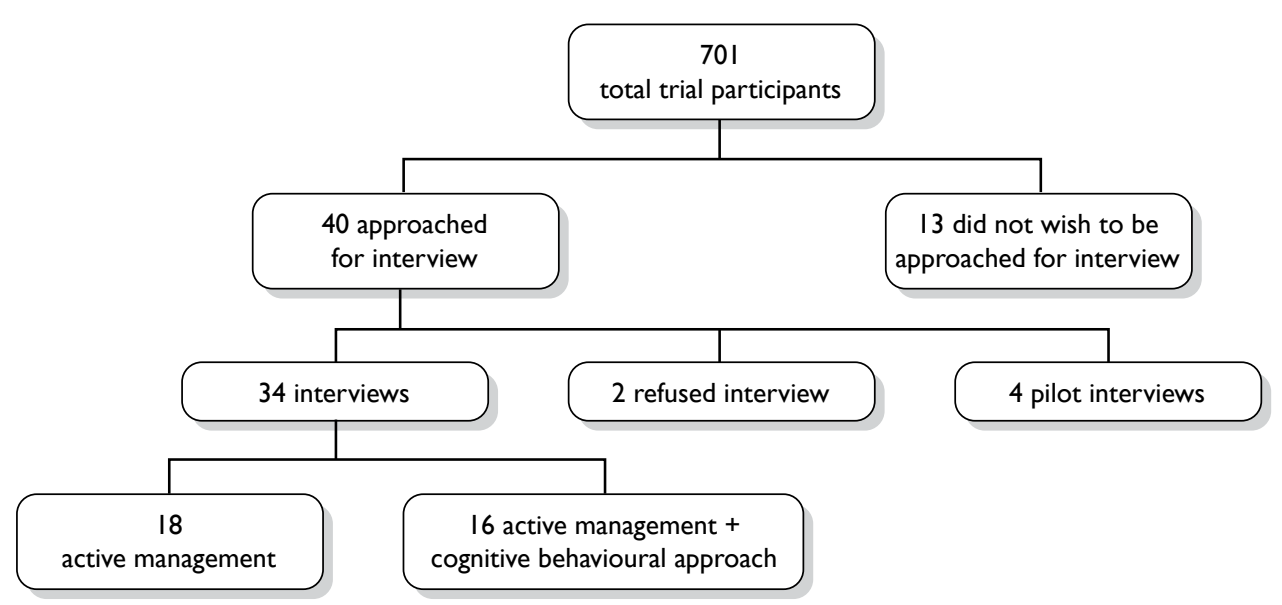

FIGURE I5 CONSORT diagram of interviews.

\section{Participant characteristics}

Table 34 gives the demographic characteristics of the interviewees. The range of RMQ and FABQ pain severity scores was similar to the clinical trial sample, as was the proportion of males and females and people from ethnic minorities. There was a wide age range among the interviewees. In comparison with the trial, a greater proportion of interviewees left school at 16 years or less, suggesting that we were successful in accessing those of lower educational attainment.

\section{Description of intervention}

\section{Attendance}

Of the $16 \mathrm{AM}+\mathrm{CBA}$ interviewees, nine attended assessment plus at least three group sessions, a slightly lower proportion than that in the whole trial. Table 35 lists the attendance of all interviewees for each group session.

Participants were considered to have received the intervention if they attended three or more group sessions. Four of the 16 interviewees randomised to the CBA did not attend any of the group sessions: two because of illness, one because of family commitments and one because after the initial assessment the interviewee decided the group would not be useful. Three interviewees attended the first one or two sessions only: two because of work commitments and one because of family commitments. Of these, one felt the group was not useful to him and two said the sessions were useful. The remaining nine attended three or more sessions but only one attended all six sessions. Absences were for a variety of reasons, such as a

\section{The interview}

A semi-structured interview schedule was designed and developed by undertaking four pilot interviews, which were not subsequently used in the analysis. In the final interview schedule, interviewees were asked about their lives in general and their back pain, as this formed the context for understanding their lived experience before and after the intervention.

The following questions regarding the interventions were asked of all interviewees:

- Which treatment did you have on this trial? Could you tell me what you thought of that?

- Were you given any written material to do with your back?

Questions asked only of the AM+CBA interviewees were:

- What do you think about physical activity for your back?

- How do you decide when to go back to work/ normal activities?

- How do you feel about the whole process of back skills training?

- How did you feel about the logbooks and goal setting?

- How did you feel about being in a group?

- Any other comments about the sessions?

Additional questions were included in the interview schedule and are provided in Appendix 14. However, these were not part of the commissioned project and hence are not reported here. 
TABLE 34 Characteristics of interviewees

\begin{tabular}{|c|c|c|c|c|c|c|}
\hline & $\begin{array}{l}\text { All BeST trial } \\
\text { participants (\%) }\end{array}$ & $A M(\%)$ & $\begin{array}{l}A M+C B A \\
(\%)\end{array}$ & $\begin{array}{l}\text { All interview } \\
\text { participants } \\
(\%)\end{array}$ & $\begin{array}{l}\text { Interview } \\
\text { AM (\%) }\end{array}$ & $\begin{array}{l}\text { Interview } \\
\text { AM+CBA (\%) }\end{array}$ \\
\hline$N$ & 701 & 233 & 468 & 34 & 18 & 16 \\
\hline Age range (years) & $18-85$ & $19-85$ & $18-85$ & $19-76$ & $19-76$ & $28-69$ \\
\hline \multicolumn{7}{|c|}{ Roland Morris Disability Questionnaire baseline scores } \\
\hline Mean & 8.7 & 8.5 & 8.8 & 8.7 & 8.4 & 9.1 \\
\hline Range & $0-23$ & $0-22$ & $0-23$ & $2-19$ & $3-18$ & $2-19$ \\
\hline Missing & 1 & 1 & 0 & 0 & 0 & 0 \\
\hline \multicolumn{7}{|c|}{ Fear Avoidance Beliefs Questionnaire baseline scores } \\
\hline Mean & 13.8 & 14.2 & 13.6 & 14.2 & 14.9 & 13.3 \\
\hline Range & $0-24$ & $0-24$ & $0-24$ & $0-24$ & $6-24$ & $0-24$ \\
\hline Missing & 39 & 14 & 25 & 1 & 0 & 1 \\
\hline \multicolumn{7}{|l|}{ Gender } \\
\hline Male & $284(4 I)$ & $90(39)$ & $189(40)$ & $16(47)$ & $8(44)$ & $8(50)$ \\
\hline Female & $415(59)$ & $142(60)$ & $278(60)$ & $18(53)$ & $10(57)$ & $8(50)$ \\
\hline Missing & $2(0)$ & I (0) & I (0) & $0(0)$ & $0(0)$ & $0(0)$ \\
\hline \multicolumn{7}{|c|}{ Severity of back pain } \\
\hline Very/extremely & $319(46)$ & $103(44)$ & $216(46)$ & $15(44)$ & 7 (39) & $8(50)$ \\
\hline Moderate & $382(54)$ & $130(56)$ & $252(54)$ & $19(56)$ & II (6I) & $8(50)$ \\
\hline Missing & $2(0)$ & $0(0)$ & $2(0)$ & $0(0)$ & $0(0)$ & $0(0)$ \\
\hline \multicolumn{7}{|l|}{ Ethnicity } \\
\hline White & $618(88)$ & $206(88)$ & $412(88)$ & $31(91)$ & $17(94)$ & $14(88)$ \\
\hline Mixed & $7(\mathrm{I})$ & $3(I)$ & $4(\mathrm{I})$ & I (3) & $0(0)$ & I (6) \\
\hline Asian & $29(4)$ & $8(3)$ & $21(4)$ & $2(6)$ & $I(6)$ & I (6) \\
\hline Black & II (2) & $4(2)$ & $7(2)$ & $0(0)$ & $0(0)$ & $0(0)$ \\
\hline Chinese & $2(0)$ & $\mathrm{I}(0)$ & I (0) & $0(0)$ & $0(0)$ & $0(0)$ \\
\hline Missing & $34(5)$ & II (5) & $23(5)$ & $0(0)$ & $0(0)$ & $0(0)$ \\
\hline \multicolumn{7}{|c|}{ Age left education (years) } \\
\hline Still in education & $3(0)$ & $2(I)$ & I (0) & I (3) & $I(6)$ & $0(0)$ \\
\hline 16 or less & $387(55)$ & $|2|(52)$ & $266(57)$ & $19(56)$ & 7 (39) & $12(75)$ \\
\hline $17-19$ & $165(24)$ & $61(26)$ & $104(22)$ & $9(26)$ & $6(33)$ & $3(19)$ \\
\hline 20 or over & $113(16)$ & $40(17)$ & $73(16)$ & $5(15)$ & $4(22)$ & I (6) \\
\hline Missing & $33(5)$ & $9(4)$ & $24(5)$ & $0(0)$ & $0(0)$ & $0(0)$ \\
\hline
\end{tabular}

Prompts were used to expand on questions to elicit personal experience and opinions surrounding their LBP and the interventions. For example, if a person mentioned that a component of the intervention was not useful they were asked why, what should be changed and whether it would be useful to others. At the end of the interview, participants were asked if there was anything in the interview they wished to have removed from the audio recording, and were reminded that the interview was confidential to the research team and would be made anonymous when transcribed.

One participant had not finished the group sessions by the time of the interview, so was contacted later for a short telephone interview to ask about the last group session. One participant asked to see a copy of the transcript which was sent with a prepaid envelope for any comments (this was duly returned with minor typographical changes 
TABLE 35 Attendance at group sessions

\begin{tabular}{|c|c|c|c|c|c|c|c|c|c|}
\hline \multirow{2}{*}{$\begin{array}{l}\text { Interviewee } \\
\text { identifier }\end{array}$} & \multirow[b]{2}{*}{ Group $^{a}$} & \multicolumn{6}{|c|}{ Session } & \multirow{2}{*}{$\begin{array}{l}\text { No. of sessions } \\
\text { attended }\end{array}$} & \multirow{2}{*}{$\begin{array}{l}\text { Total no. in } \\
\text { group }\end{array}$} \\
\hline & & I & 2 & 3 & 4 & 5 & 6 & & \\
\hline 19 & A & $*$ & $x$ & $x$ & $x$ & $x$ & $x$ & 1 & 7 \\
\hline 20 & A & $*$ & $*$ & $x$ & $x$ & $x$ & $x$ & 2 & 7 \\
\hline 21 & $A$ & $*$ & $*$ & $*$ & $*$ & $*$ & $*$ & 6 & 7 \\
\hline 22 & B & $*$ & $*$ & $x$ & $*$ & $*$ & $*$ & 5 & 5 \\
\hline 23 & C & $*$ & $*$ & $*$ & $*$ & $x$ & $x$ & 4 & 6 \\
\hline 24 & C & $x$ & $*$ & $*$ & $x$ & $*$ & $x$ & 3 & 6 \\
\hline 25 & $\mathrm{D}$ & $*$ & $*$ & $x$ & $*$ & $*$ & $*$ & 5 & 7 \\
\hline 26 & $E$ & $x$ & $*$ & $*$ & $x$ & $*$ & $*$ & 4 & 6 \\
\hline 28 & B & $*$ & $*$ & $x$ & $x$ & $*$ & $*$ & 4 & 5 \\
\hline 31 & $\mathrm{~F}$ & $x$ & $*$ & $*$ & $x$ & $*$ & $*$ & 4 & 7 \\
\hline 32 & G & $*$ & $x$ & $x$ & $*$ & $*$ & $*$ & 4 & 7 \\
\hline 34 & G & $*$ & $*$ & $x$ & $x$ & $x$ & $x$ & 2 & 7 \\
\hline Total & & $9 / 12$ & $10 / 12$ & $5 / 12$ & $5 / 12$ & $8 / 12$ & $7 / 12$ & & \\
\hline
\end{tabular}

only) and another asked for a copy of any excerpt which may be used in the final report, which was sent and elicited no response.

\section{Data coding and analysis}

All interviews were carried out by one researcher (V.N.). At the time of interview the interviewer was not aware of the detailed content of the course sessions, to reduce any tendency to ask leading questions. During analysis V.N. was briefed fully on the content to enable her to understand which part of the intervention interviewees were talking about.

Audio recordings of interviews were transcribed and checked by the interviewer. All transcripts were entered into NVIVO, a computer software tool for managing qualitative data (NVIVO, QSR, Portsmouth, UK). Thematic codes were developed based on the interview schedule, the components of the intervention and issues arising in the interviews. The research team agreed codes and their definition. All data were double coded and coding was compared. Where there were inconsistencies in coding these were discussed and agreed. The data were extracted from all interviews for each coded theme relevant to the experience of AM or AM+CBA. The number of participants mentioning a theme was counted, the content of the coded data was summarised and illustrative quotes were identified for presentation in the results.

\section{Results}

The results describe the interviewees' experiences of back pain, being on the trial and their account of the interventions they received. The first section summarises the accounts of receiving AM only. We then draw on the accounts of those receiving $\mathrm{AM}+\mathrm{CBA}$ and summarise their account of the AM component, the individual assessment and the group sessions. The results include numbers of interviewees who talked about themes. Each interviewee may have talked about one or more of these themes.

\section{Active management}

This section summarises the accounts of the 18 interviewees who received AM only.

Nearly all the interviewees were familiar with the messages given in The Back Book, although none reported receiving the book previously. The advice to stay active, take regular analgesia and undertake exercise was familiar to all interviewees except one and had been gained from a variety of sources. The one interviewee who was unfamiliar with the content of The Back Book before the trial found the advice valuable.

...it [The Back Book] did involve a certain amount of details... They were easily adaptable to yourself. It wasn't something where it was a 
leaflet given to you that just has a list of things that you could do and that was it...it was done in a way that you could all sort of take bits from it that you needed. [Interviewee 5]

Seven interviewees felt that it was a 'good booklet':

Yes, it's quite a neat little document to have for a point of reference and it was quite clearly laid out and yes, I think someone coming in initially having a problem with their back would probably benefit from that, because it may give some further insight into managing back pain, but for me I had seen...I'd been given literature like that before. [Interviewee 16]

Of these, three added they had found the information reassuring:

...I think the one thing that was reassuring was that I couldn't make it any worse by carrying on doing things. [Interviewee 3]

Two of the seven also suggested that it may be useful for others in early episodes of back pain:

...if it was the first time I done it, it would have been a great book but I've seen a lot of it before. [Interviewee 18]

Two-thirds of the interviewees reported that the advice in The Back Book was common sense, nothing new or similar to other advice they had received in the past:

I thought it was common sense things really, I really did and I thought 'well it's not telling you anything that isn't real common sense'. [Interviewee 14]

Well, I've been...it's exactly...I've been told and I've had things like that for years, so I do know...yes, I read it and it was in a way nothing new for me. [Interviewee 17]

Well, I read it but it was all what I'd sort of read before as well when I've been to clinics and surgeries and things like that. [Interviewee 6]

However, six interviewees could not remember receiving The Back Book in the trial at all, even when prompted. One of these participants also said that he had 'lost interest' [Interviewee 9] after not being chosen for the group intervention and felt that this was part of the reason that he could not remember the book.

The Back Book and AM session was considered too general or unhelpful by two interviewees who wanted specific treatments.

Like it doesn't tell you what you've actually gotta do with your back. [Interviewee 8]

I was hoping that I was going to get some... maybe go on an exercise course or something like that you know? You know, I mean it's interesting just talking but it doesn't really do anything does it? [Interviewee 10]

\section{Active management and cognitive behavioural approach group intervention}

This section summarises the accounts of the 16 interviewees who were in the AM+CBA arm of the trial. Of these, 12 attended at least one group session.

Two or more of the key messages of the CBA group intervention were picked up by 11 of the 12 interviewees attending at least one group session. Six of these had picked up three or more of the key messages.

Details of the interviewees' accounts are reported in the following sections covering the AM session, the initial assessment and the CBA group sessions.

\section{Active management experience for the participants receiving CBA}

All $16 \mathrm{AM}+\mathrm{CBA}$ interviewees received the AM session after randomisation. However, these interviewees' descriptions of the AM part of the intervention were more general and sometimes vague, compared with the AM interviewees. The parts of the intervention that followed this session were remembered more clearly. When asked about the AM component of the intervention, all 16 interviewees gave a response but often needed prompting. Some gave more than one comment in different themes and the themes were similar to those reported by participants randomised to AM.

Eight interviewees felt that The Back Book gave 'common sense' information or that they already knew the information from other sources. One of these interviewees compared The Back Book to the BeST intervention folder given at assessment: 
It was interesting but it was straightforward about what it was. I think it was a good opener to whether you went on the programme...you know, if you were lucky enough to be chosen or whether you just had the booklet and it did explain backs and that but I preferred the folder. Yeah, the folder sticks in your mind more than the little book. [Interviewee 24]

Four interviewees could not remember anything about the AM session's content and four mixed up the BeST intervention folder given at assessment with The Back Book.

One participant thought The Back Book was unhelpful:

I did feel that...they didn't quite understand what it was like to have back pain...I just wondered if any of them had actually suffered any back pain and if they really understood exactly what it was like to have this...just this awful feeling really of never ever going to be any better. [Interviewee 32]

\section{Initial assessment}

All 16 interviewees attended the initial assessment before commencing the six group sessions. Their accounts of each of the assessment components are described below: exercises, goal setting, the BeST intervention folder and the relationship with the therapist.

\section{Exercises}

This section draws together interviewee accounts of exercise both at the initial assessment and throughout the group sessions, as the interviewees did not distinguish clearly between exercises at initial assessment and exercise discussed during group sessions.

Exercises were talked about and considered of benefit by nine interviewees:

Overall I feel they've been helpful,...really helpful...I was a bit worried until...about my knee, but now I know it's my back I can concentrate on that more. I've...because I cut all the exercises out that I...but I've started them up again and touch wood they're working. [Interviewee 22]

Exercises had been incorporated into their lives to good effect by seven of the nine interviewees:
I had the booklet (folder) with all the exercises, which is very good I have to say. They are good strengthening exercises, good flexible exercises, which in the training that I do...I do quite a lot of flexing and quite a lot of exercise anyway and I incorporate the booklet (folder) into that as well now, so from my point of view that's been a benefit. [Interviewee 19]

Exercises had increased the ability to cope for two of the nine interviewees:

...since going on the course, try and exercise; try and move it, try and free it up, whereas previously I'd have followed the...you know, lay down, rested it and I sort of picked up on that and it did make a difference the other day for sure. I went from absolute agony to...I actually phoned in work to say I wasn't coming in and then an hour and a half later I phoned in and said 'Yes, ok I'll come in I'm ok I'm better.' Not 'fixed' but better able to cope. [Interviewee 20]

...but I feel more confident that I'll be able to...if I do get it bad, get over it with the exercises and the same with the positive side as well, you know and sometimes it hurts, but you've got to go through it... [Interviewee 21]

The flexibility of how the exercises were taught was specifically mentioned and appreciated by four interviewees from the whole sample of 16 :

I didn't really have any expectations. I thought it might have been harder in the exercises and I'm sort of thinking 'oh God, are they going to get me down on my hands and knees trying to do all the...' but it was very easy [therapist's name] was very good how she explained things and she saw to at least one of (us) each week to make sure that we were doing the exercises. If we had a problem with any of them she would help us through it... [Interviewee 24]

One participant had not done the exercises they had been shown, but said they might in the future:

...I'm not a natural exerciser so it's very hard to go from doing absolutely nothing to doing something you know on a regular basis...now I didn't do them but I know I could...I can if I wanted to, highly unlikely but if I wanted to I could do it but I would have said it was worth while. [Interviewee 25] 
Two interviewees felt that they would have liked the opportunity to exercise and be shown how to do the exercise in the 'correct way':

they were (not) going to actually...physically going to show you the exercises that you need to do...I think is sort of a real key to you know keeping flexibility right, making sure you are doing the exercise right. Because some people can do an exercise completely wrong and they're not stretching the muscle they're supposed to be stretching. [Interviewee 19]

It would better that...if the exercises could actually be conducted in the sessions...It's not that we weren't allowed to do it, I should say. It's just that we weren't given the opportunity to do the exercise. [Interviewee 31]

One interviewee said that the exercises were ineffective for them. This interviewee was unable to attend any of the groups:

...she gave me a set of exercises to do but they didn't seem to help you know, stretches and exercises but that didn't seem to help. [Interviewee 33]

One interviewee was already doing a lot of exercise:

I'm already exercising more than most people exercise. [Interviewee 23]

\section{Goal setting}

Of the 16 interviewees, 13 talked about setting goals collaboratively with the therapist at the initial assessment.

Goal setting was popular with six interviewees:

What mine (goal) was, was to walk to the shop and back and I did it within the first couple of weeks because the car was off the road so I had to do it and I was so pleased when I'd done it that I actually made it there and back. [Interviewee 24]

Well I have joined the gym so that was one of my goals to go back and do that, but then I've got to work on that. [Interviewee 32]

Goal setting was already being used by a further two interviewees:

Many things I learned on the course I already worked out myself over time...I've achieved the goal that I actually set on the course with the swimming. [Interviewee 23]

Goal setting was not used by three interviewees:

I'm not bothered about goal setting.

[Interviewee 22]

I felt I wasn't particularly bad enough to have to set goals, because it isn't restricting me in the way it was probably affecting other people. [Interviewee 26]

I mean I'd like to go on it every day and do ' $x$ ' amount of time on it, but at the moment like I say, I actually haven't but that was one of my goals. [Interviewee 30]

Two interviewees felt that goal setting would be good for others:

For some people I'm sure it's good, but for me personally...I was happier just doing it my way in the end. [Interviewee 20]

\section{BeST intervention folder}

Seven interviewees mentioned the folder.

Five interviewees found the folder good, of whom three specifically mentioned exercises and one used it for reference:

It showed you exercises, it explained back pain, that going round in a circle. It gave me goals, what to deal with um...and once you'd done with one goal...you know, 'do you want to lead on to another goal?' With the exercises you started at one level and if you wanted to carry on different levels but each week we were given two pages about what we talked about that day which was very good. [Interviewee 24]

Yes I did actually think it was quite useful but yes it has been quite good and I have referred to it strangely enough. [Interviewee 25]

No one used the logbooks.

\section{Relationship with therapist}

The relationship with the therapist at the initial assessment was described as positive or helpful by four interviewees:

She was very good, everything was explained. She asked...she waited for us to answer and if we had any questions she'd listen to us and 
she'd...you didn't feel as though you were being rushed. [Interviewee 24]

I felt quite uplifted when I came out actually, that somebody perhaps wanted to help me. I don't know. Whether just that feeling of, 'someone's listening'. [Interviewee 32]

\section{Group sessions}

Out of the 16 interviewees who attended for the initial assessment only 12 attended one or more group sessions. Their accounts of the group sessions are presented under the following themes: change in fear avoidance, value of interaction, pacing, pain education, relaxation and reinforcement. The final section reports the negative comments about the group sessions.

\section{Change in fear avoidance}

The most marked change in attitudes, beliefs and behaviours attributed to the group sessions was a decrease in fear avoidance, described by 10 interviewees:

I always assumed that if I was in pain that I was going to damage it (the back)...I'll take paracetamol or the Ibuleve gel around the area and since going on the course, try and exercise; try and move it, try and free it up, whereas previously I'd have followed the...you know, lay down, rested it and I sort of picked up on that and it did make a difference the other day for sure. [Interviewee 20]

I would take it easy and I will try and restrict the amount of heavy lifting I done I wouldn't lift it...I were conscious of not damaging it... because I was frightened to do these because what's the point of exercising and then doing your back in like? I'm not so worried about it now. [Interviewee 21]

You're afraid that if you push yourself too far that maybe you're doing more damage to your back...but as I say, that was good in the fact that what it showed me is that I can push myself that little bit further with the pain, and it's not actually doing any damage. [Interviewee 31]

The only things that have changed from the trial is that I will attempt more things...even though it hurts. [Interviewee 23]

I'd probably say it's (back pain) on a similar scale of what it was, but not...it's not emphasised by worry or fear. It's not being fed.
I'm not sitting there thinking about my back constantly basically, thinking 'Oh well I can't do that. Can I lift that?' I'd just go and lift it and lift it carefully. [Interviewee 26]

One interviewee talked of still being fear avoidant:

I like to do it if I can but I'm wary of it that is the main thing. I'm frightened of it...it sounds silly but I'd like to put it in a washing machine, wash it and put it back new which will never happen but yeah I'm frightened to do anything new with it in case it does it, I'll do it bit by bit...I prefer to do it bit by bit than to see how far I can go...years ago I'd have gone and done a whole lot but now you won't because you're frightened of your back. [Interviewee 24]

An understanding of cycles of pain and the link with fear avoidance was expressed by five interviewees:

I did tend to protect my back, I did tend to... but obviously if you're not going to use your back then you're going to lose your back, you know and you get into the circles and the whole thing made a lot of sense really. [Interviewee 21]

I guess that is a good thing to do, you know, to use the exercises because it is an absolute vicious circle. You don't exercise because your back hurts which in turn weakens your muscle which in turn makes your back bad and you're on the treadmill really so it's difficult. [Interviewee 25]

What I've tended to do as a result of the sessions is even though my back is aching I've pushed myself and not given in. I've gone on and done something and like...you know... I've gone down and taken her (niece) to the swimming baths. [Interviewee 31]

\section{Value of interaction in the group sessions}

The opportunity to share views and experiences with the group was valued by nine interviewees:

The difficulties they were having were all quite common with the difficulties I was having and from that, I found it quite comforting. [Interviewee 31]

Sometimes you can pick up more from the people because they've done things or 
they get round things in a different way. [Interviewee 31]

That was...yes, just listening to what people did, what treatments they'd had yes, but other treatments that everybody had tried and what effectiveness that had had. [Interviewee 20]

I found it very good because we were...because when you are by yourself you're the only one with this problem and nobody else knows what you're going through. Because you're living with a family group and they might have a back ache but they don't have the back like we do... it was reassuring that you are not by yourself. [Interviewee 24]

One participant didn't feel comfortable in a group and attended only one session:

I wasn't comfortable with...(the group)...it's just because you feel a bit silly you know?... It's not the normal approach that you do. It's normally like us one to one or whatever and you feel like you can say what you want to say, or you don't want to sound stupid by saying something really simple that's not connected with the questions they're asking. [Interviewee 19]

Three interviewees gave accounts of why their group dynamics may not have worked as well as they might have. These were due to poor attendance or individuals not participating or monopolising the group.

Comparison was made between the interviewee and other group members by six participants, of whom four described their back pain as less severe than others:

Mine wasn't so bad compared to like some of them. [Interviewee 28]

I'm not a particularly bad case. When I was sat with the three guys from the group I knew...I was sitting there thinking 'Well, I'm not this... quite this bad.'...I thought 'Well, I'm not in his category', which obviously made me feel a lot better. [Interviewee 26]

I realise how lucky I was, because some people have suffered an awful long time. [Interviewee 19]

\section{Pacing}

The technique of pacing of activities was mentioned by three interviewees:

(If I) build up reasonably slow, then there's no reason why I can't have a go at anything, which is what I'm in the process of hopefully doing now. [Interviewee 21]

Difficulty in applying pacing in their lives was described by two interviewees:

I know I shouldn't (over) do it but you don't know how many good days you're going to get so... [Interviewee 22]

Although they said on the research you need to get a happy medium when you've got a bad back problem...they wanted you to get... so that you could do as much on a bad day as you could on a good day, that is impossible... because as I say sometimes you're totally incapable even to get out of bed sometimes so you can't do say a walk to the end of the road and back which they wanted you to do and I found that sort of, good idea but impossible to do. [Interviewee 24]

\section{Pain education}

Understanding the psychological component of pain was useful for three interviewees:

The psychological effects...because that's actually quite important because it does have a big effect on people. I'm sure it...well, certainly on me...I mean mostly in the evenings when I'm watching television I'm uncomfortable for most of the evening to be honest and I just keep shifting my position...Well, mainly what they were telling us at this back clinic. You distract yourself. That's really the only way. I get up, I walk round, I probably get myself a drink, watch television and get absorbed in something perhaps for an hour and I'm not thinking about it. During the daytime I just am busy and that takes my mind off it. [Interviewee 32]

However one participant did not find the pain advice useful:

That's something to do with mental high pain and I was like well yes if you want to know more... deeper into backs it's great but that was irrelevant for what...for me if you like... it weren't getting to the point you know?

[Interviewee 34] 


\section{Relaxation techniques}

Relaxation was described as useful by two interviewees of whom one had used it previously:

I think it was good, I did pick up things and I mean we talked about relaxation and that was quite good and [therapist] went over some relaxation techniques, I found those useful. [Interviewee 25]

\section{Reinforcement}

The group sessions reinforced activities and beliefs they had already learnt about. These included exercise, goal setting, relaxation and pacing. One participant felt that the group had confirmed that there was nothing new that they had not already tried.

\section{Dissatisfaction with the treatment}

Four people made negative comments about the groups. The negative comments are described fully below.

One interviewee attended one group session only and stopped because of work commitments and because he had expected medical tests or a definitive diagnosis. He also stated a preference for a one-to-one treatment rather than a group and said:

Going on the actual course... although I missed quite a lot because the first week I went I felt it was a little bit patronising I have to say, a little bit like going back to school and that sort of thing. [Interviewee 19]

Another interviewee said that they 'knew everything already', the course had confirmed this and they felt that there was a problem with the pitch and delivery:

You've got it pitched incorrectly...I mean the person who was taking it...she was a really sweetie she's lovely, but...and she was following instructions that they've all got from these books, but it's pitched really. I mean I should think it's pitched for a teenager...It's not pitched for intelligent adults... Either the pace or the way they actually talk to you or...you know, you need to be much more...For a start you need a lot more examples up your own sleeves in your own heads that you can bring out when you try to get a group to talk, you know. I mean you need a lot of teaching skills really and you haven't got them...It's partially the content and it's partially the way it's put over. It's both. [Interviewee 23]

One interviewee did not like the therapist's approach:

I didn't like him. I thought it was patronising, arrogant and I thought he was a $* * * *$ to be honest with you because about what goals I had and they talk to you like you're a kid. Do you know what I mean, which I didn't like?... I turned round and I said I wanted to run a marathon and he slapped me down.

[Interviewee 28]

This interviewee also knew the information given in the group.

An interviewee who only attended two sessions because of work commitments and felt they had stopped learning from the group sessions:

I missed out two or three sessions.... and some of it I found a little bit boring...Well like the.. well...when they were saying right what sort of pain is there, there's severe pain, there's mediocre pain, there's little pain....and I was like 'well yes if you want to know more...deeper into backs it's great but that was irrelevant for what...for me if you like' but sometimes it's nice to talk to people about their back but... it weren't getting to the point you know?... And it was great, I mean six sessions I realised I did three or four and then the sessions I did do...it was great but then I said 'well I'm not learning here' and you think to make my back better or though it was interesting what other people do so it...if you give it a mark out of 10,5 or 6 . Yes for the course generally...The first one (assessment) the back 10 out of 10 and I'd go to that again...that was brilliant. [Interviewee 34]

These four people were asked how useful they felt the groups would be for other people. Three felt that other people may benefit from this type of intervention; the other did not comment.

\section{Conclusion}

The CBA group intervention was added value for the trial participants over AM. Almost all participants were already familiar with the AM approach. 
Most of the interviewees in the CBA intervention arm of the trial and who attended at least one session picked up key messages from the intervention and two-thirds talked about a reduction in fear avoidance and changes in their behaviour. Over half found the exercises helpful and many of these had incorporated exercise into their daily lives. Other aspects of the intervention were useful to some and not others, suggesting that offering the range of techniques is important.

Although participants of the BeST trial were considered to have received the intervention only if they had attended three or more sessions, the interview data suggest that fewer than three sessions may still be useful for some participants.

A minority of interviewees were unhappy with certain aspects of the group intervention and several of these felt they knew the content of the intervention already.

Issues that were raised by the interviews that should be considered in translating the intervention into practice were as follows:

- The individual assessment, although an unusual component of CBA, sets the agenda for the groups, begins the CBA and allows a relationship to be established with the therapist. It is an important element of the intervention.

- Exercises were considered an important component by most interviewees and demonstration and tailoring of exercises was valued. Great emphasis could be placed on tailoring and demonstrating exercises during the sessions.

- Participants can benefit even if they choose to use only certain aspects of the intervention or attend a limited number of groups.

Translation into practice should consider giving participants more information about the sessions ahead of time, so that people are able to choose what to engage with and when. Although not mentioned specifically, a rolling programme of group meetings may mean that participants have a greater choice about being able to join when they choose.

- Group sessions where participants share experiences can provide them with reassurance, lessen isolation and enable participants to learn strategies from each other.

- Consider increasing training for therapists in group facilitation to increase sharing of experiences. 



\section{Chapter 6 Economic analysis}

\section{Introduction}

We aimed to assess the cost-utility of adding a CBA programme to AM. Our primary outcome was cost-utility expressed as the incremental cost-utility ratio (ICUR), ${ }^{131}$ the shadow 'price' of CBA, which indicates the cost of gaining one additional QALY relative to the current therapy.

We assessed cost-utility over a 12-month time frame, and the base year was 2008. Costs were expressed in UK pounds (£) and actualised to 2008 using the Office of National Statistics Retail Price Index ${ }^{132}$ where required. Neither costs nor outcomes were discounted as the time frame for the analysis was 12 months.

We considered cost-utility from both the UK NHS and the general health-care perspective. ${ }^{131}$ The NHS perspective included all health-care costs supported by the NHS to deliver AM or AM+CBA and to provide health care associated with back pain treatment and symptoms in the 12-month period after randomisation. The health-care perspective is broader and included both the total health-care costs to the NHS and the cost of privately purchased goods and services (medical and non-medical) related to LBP and private transport.

The base-case analysis was based on the withintrial resource consumption. To illustrate the generalisability of the cost-effectiveness analysis, we developed an alternative cost-utility scenario based on the potential resource consumption for the CBA programme delivered within NHS structures where therapists are almost entirely based at one site (and hence there is no need to allow for travel time and expenses). The within-trial analysis was the primary economic analysis and is presented in this chapter. The generalised NHS scenario was a secondary analysis and appears in Appendix 15.

\section{Data}

\section{Outcome data}

Health outcomes were expressed as QALYs calculated from utility weights ${ }^{133}$ estimated from preference-based questionnaires (EQ-5D) completed at baseline, 3, 6 and 12 months.

The EQ- $5 \mathrm{D}^{107}$ is a generic measure of health status that includes five health dimensions: mobility, selfcare, usual activities (e.g. work, study, housework, family or leisure activities), pain/discomfort and depression/anxiety. For each dimension, the respondent is asked to rate the extent of their problem, choosing between 'no problem', 'some problems' or 'unable to perform'. The combination of all potential responses gives rise to 243 health states ranging from full health to worst possible health state.

\section{Resource utilisation data}

The resources used to deliver the CBA were obtained from administration and visit records. CBA resource use included duration of the assessment visit, number and duration of scheduled CBA sessions (contact time) and non-contact time, equipment, consumables, educational materials and items and resources used during the training of CBA therapists. Non-contact time included the time used to contact participants and set up the sessions, writing clinical notes, administration and record-keeping. Support and supervision time provided by the intervention support team were incorporated. Travel time was calculated for therapists seconded to the programme using actual travel time reimbursed by the trial.

We collected other health-care resource use and participant costs from participant questionnaires at baseline, 3, 6 and 12 months. Participants were asked to keep a record of type and quantity of each resource item used, for services provided by the NHS and for services either privately purchased or funded by private health insurance.

Other NHS resource use data included the number of contacts with GPs, nurses, physiotherapists, psychologists, other health-care consultations, diagnostic tests [including X-ray, magnetic resonance imaging (MRI) scan, computerised tomography (CT) scan and blood tests], accident and emergency (A\&E) attendances, and hospital admissions. 
We also collected data on the provision of drugs used in the treatment of back pain from participants' questionnaires. These included painkillers, anti-inflammatory tablets, antiinflammatory gels and creams, sleeping tablets and antidepressants, and other drugs indicated by participants as having been prescribed for back pain.

We sought data on out-of-pocket expenses, as well as on back care sourced from the private (nonNHS) sector. This included the resource use and costs of services such as aromatherapy, chiropractic, counselling, massage, private orthopaedic surgery, osteopathy, psychology, physiotherapy and the number of items and cost of any equipment item, housing adaptations and aids. We asked participants to indicate the type and quantity and, where appropriate, unit cost for each item of privately purchased health-care resources. We also asked participants to indicate where these costs had been paid for either in total or in part by a private insurance policy.

We verified both NHS and private hospital admissions data. A researcher (E.W.) contacted the patients who had declared any hospital admission and collected data on cause of admission, ward of admission (to determine the Health Resource Group), procedures received and length of stay.

\section{Unit costs}

The total cost of health care was calculated by multiplying resource use data by their respective unit costs. Unit costs were the cost of $\mathrm{AM}$ or $\mathrm{AM}+\mathrm{CBA}$, the cost of primary care and community-based services, the cost of hospitalbased services, admissions and A\&E services, the cost of drug prescriptions, and the cost of private health-care items and equipment. All unit costs reported in this section are 2006 prices, with the exception of private consultations and tests (Table 36), and were actualised to the year 2008 for the cost-utility analysis using the appropriate inflation index.

\section{NHS health-care unit costs}

Unit costs for any of the resources consumed and consultations within the NHS were obtained from published sources pertinent to the UK. ${ }^{134-136}$ Unit costs for these items are reported in Table 36.

Unit costs for pharmacological agents were the average cost for one prescription of each drug type consumed in the trial, including painkillers, anti-inflammatory drugs, anti-inflammatory gels and creams, sleeping tablets and antidepressants. Unit costs were calculated based on the typical drugs commonly prescribed for back pain, based on a consensus exercise amongst six academic GPs working in the localities from which we recruited the sample. The average cost of the prescriptions was then computed from the Prescription Cost Analysis (PCA) database. ${ }^{136}$

Participants were asked to report if they were dispensing any drugs in addition to the categories described above. Costs for these additional drugs were calculated using the frequency of use reported

TABLE 36 Unit costs for NHS consultations and drugs (2006 prices)

\begin{tabular}{|c|c|c|}
\hline Item & Unit cost ( $(\epsilon)$ & Source \\
\hline GP visit (surgery) & 34.00 & Curtis $2007^{134}$ \\
\hline Nurse visit (surgery) & 9.00 & Curtis $2007^{134}$ \\
\hline Physiotherapy visit & 31.80 & Curtis $2007^{134}$ \\
\hline Outpatient consultation (back pain problems) & 112.70 & NHS Reference Costs ${ }^{135}$ \\
\hline Psychologist consultation & 67.00 & Curtis $2007^{134}$ \\
\hline Painkillers & 4.57 & PCA database $\mathrm{e}^{136}$ \\
\hline Anti-inflammatory drugs & 8.22 & PCA database $\mathrm{e}^{136}$ \\
\hline Gels, creams and ointments & 5.75 & PCA database $\mathrm{e}^{136}$ \\
\hline Sleeping pills & 3.66 & PCA database ${ }^{136}$ \\
\hline Antidepressants & 5.86 & PCA database ${ }^{136}$ \\
\hline
\end{tabular}


by respondents, when available, or assuming occasional consumption of one dispensing unit (prescription). These additional items were yearly treatment with paracetamol at $£ 12.00$ assuming one box per week, pain control (fentanyl) patches, at the cost of $£ 86.70$ per prescription, and muscle relaxants at $£ 11.90$ per prescription. ${ }^{136}$

The costs of diagnostic tests and of hospital and A\&E admissions were obtained from the NHS Reference Costs database. ${ }^{135}$ The cost of blood tests was calculated using the average cost of blood tests (Speciality codes DAP823 and DAP839). This was $£ 3.10$. The costs of other diagnostics were CT scan (£117.90), MRI scan (£203.80) and X-ray (£28.00).

The unit cost of hospital admissions was obtained from the category HD26C Musculoskeletal Signs and Symptoms Without Complications. The cost of a day case was $£ 529.50$ and the cost of an extended-stay case was $£ 572.00$. The cost of an A\&E attendance without a subsequent admission was $£ 170.10$.

\section{Private health-care unit costs}

Private costs were estimated based on resource consumption indicated in participants' questionnaires multiplied by the respective unit cost.

The unit costs of private consultations included a range of services (shown in Table 37). These costs were obtained from a telephone survey of six providers of each type of service, based in Coventry, Manchester, Newcastle, Essex, Liverpool and London, conducted between 1 February and 5 February 2008 (Table 37).

Private costs also included the cost of privately paid diagnostic tests, including X-ray, MRI scan, CT scan and blood testing. Unit costs for private tests and diagnostics were obtained from the same telephone survey and are detailed in Table 37.

Medications (painkillers, anti-inflammatory drugs, anti-inflammatory gels and creams, sleeping tablets and antidepressants) purchased privately were valued at the same NHS cost for similar preparations obtained from the PCA database. ${ }^{136}$

TABLE 37 Unit costs of community- and hospital-based private consultations

\begin{tabular}{|c|c|c|}
\hline Item & Unit cost (E) & Source \\
\hline \multicolumn{3}{|l|}{ Hospital-based fees } \\
\hline $\begin{array}{l}\text { Outpatient consultation with } \\
\text { NHS consultant }\end{array}$ & 182.50 & \multirow{5}{*}{$\begin{array}{l}\text { Phone survey of six consultants' private consultation fees for patients } \\
\text { with LBP, at BMI Alexandra Hospital (Cheadle); BMI Meriden Hospital } \\
\text { (Coventry); BMI Sarum Road Hospital (Winchester); Spire Bristol; Spire } \\
\text { Gatwick Park; Spire Leicester. Conducted in February 2008. Estimates } \\
\text { are the average cost }\end{array}$} \\
\hline X-ray & 86.20 & \\
\hline CT scan & 551.60 & \\
\hline MRI scan & 597.90 & \\
\hline Blood tests & 109.80 & \\
\hline \multicolumn{3}{|l|}{ Community-based fees } \\
\hline Physiotherapy & 38.20 & \multirow{9}{*}{$\begin{array}{l}\text { Phone survey of six specialists' private consultation fees for patients } \\
\text { with LBP, at Coventry, Essex, Liverpool, London, Manchester and } \\
\text { Newcastle. Conducted between I February and } 5 \text { February } 2008 . \\
\text { Estimates are the average cost }\end{array}$} \\
\hline Osteopath & 44.30 & \\
\hline Chiropractor & 35.30 & \\
\hline Psychologist & 70.40 & \\
\hline Counsellor & 50.00 & \\
\hline Massage therapist & 24.80 & \\
\hline Aromatherapist & 25.30 & \\
\hline Dietician & 55.80 & \\
\hline Acupuncture & 34.50 & \\
\hline
\end{tabular}


For hospital consultations, participants were asked to indicate whether the cost was disbursed directly by the participant or reimbursed by health insurance, while equipment costs, housing adaptations and aids, all purchased privately, were estimated using the amounts declared by participants only. Finally, we computed participant travel cost for all visits, consultations and hospital admissions declared as either NHS or private services using unit costs obtained from one published study for the UK. ${ }^{137}$ Travel to community-based services was estimated at $£ 3.50$ and that to hospital-based services was estimated at $£ 6.50$ per trip.

\section{The cost of cognitive behavioural therapy in the randomised controlled study (within-trial analysis)}

Unit costs for the AM+CBA intervention were estimated from the trial data, using resource use described above and other staff cost information retrieved from published sources. ${ }^{134}$ The unit costs for the components of the CBA programme were staff salary costs (including overheads and capital costs), training costs, consumables and equipment and travel costs. The total cost per CBA cycle was then computed for each group and allocated to each participant in relation to the number of sessions attended and the number of participants per session.

In the cost-utility analysis, CBA was estimated using the actual resource used to deliver the programme, i.e. the total working time provided within the context of the trial. Staff salary costs of one CBA cycle were then calculated as the cost of the total number of contact and non-contact hours spent by a therapist to deliver CBA multiplied by the normal salaried staff hourly rate.

The effective staff hourly rates paid in the trial were not likely to represent the normal salaried cost of staff. As with many trials, some of the activities were paid for under casual working arrangements. Although these include a holiday enhancement, they do not account for the broader costs of employment if undertaken on a salaried basis. Therefore the total normal staff hourly cost was computed, inflating the effective salary rates paid in the trial to include gross salary, employers' and overhead costs, capital costs and capital overheads. Gross salaries were calculated based on effective seniority for each therapist combined with published NHS pay scales. ${ }^{138}$ Salaries were inflated with $8 \%$ employers' National Insurance costs and $14 \%$ for employers' contribution to superannuation pay. ${ }^{134}$ Salaries were then inflated at the year 2008 using percentage pay increases for the year 2006-7 $(5.5 \%)$ reported in Curtis. ${ }^{134}$ As salary increase data for the year 2007-8 are not available the same percentage increase was compounded for that period.

Overheads were applied to total staff time in a proportion of $5 \%$ of the salary for direct revenue overheads and $£ 2904$ per year for indirect overheads. ${ }^{134}$ Capital costs and overheads were valued at $£ 2752$ per year. ${ }^{134}$ Hourly rates were then computed from the total salary costs, assuming a total working time of 5 days and 52 weeks, less 27-33 days of holiday per year, depending on the therapist's salary band, 8 days for statutory holidays and an average of 12 days' sick leave and 3 days' training. ${ }^{138}$ Hourly rates used in the costeffectiveness are summarised in Table 38.

Finally, the total salary cost of one cycle of CBA was computed by multiplying the number of worked hours per cycle by the therapists' normal hourly salary rates. The total working time was the sum of contact and non-contact time observed for each therapist in the trial. Therapists' travel time was included in non-contact time, as the intervention was delivered by therapists seconded to the trial from other institutions where they had a permanent position.

The total cost of training, supervision, consumables and equipment was allocated top-down to each CBA cycle, based on the number of groups in the CBA programme.

The cost of consumables and equipment was computed by multiplying the number of items bought for the trial by their acquisition costs.

Training costs were estimated by summing the cost of external lecturing fees, intervention materials, lecture room fees, and residential expenses, including accommodation, travel and subsistence costs. Salary costs of therapists attending training were not added, to avoid double counting as working time spent on training was already included in the computation of the therapists' hourly rate. Training costs were allocated by $\mathrm{CB}$ cycle rather than as a component of salaries, as we requested that the staff did not deliver $\mathrm{CB}$ therapy outside the trial context. The CBA training course 
TABLE 38 Hourly salary rates calculated for the CBA programme

\begin{tabular}{llllll}
\hline Therapist & Band & Base salary $(\boldsymbol{E})$ & Staff total cost $(\boldsymbol{\epsilon})^{\mathbf{a}}$ & Days worked per year & Hourly rate $(\boldsymbol{\epsilon})$ \\
\hline I & $6 / 4$ & 23,789 & 39,283 & 210 & 24.90 \\
2 & $6 / 7$ & 26,720 & 43,426 & 210 & 27.60 \\
3 & $6 / 8$ & 27,622 & 44,701 & 210 & 28.40 \\
4 & $6 / 9$ & 28,524 & 45,976 & 210 & 29.20 \\
5,6 & $6 / I 1$ & 31,004 & 49,481 & 210 & 31.40 \\
$7-10$ & $7 / 7$ & 31,906 & 50,756 & 208 & 32.50 \\
II-19 & $7 / I 1$ & 36,416 & 57,132 & 204 & 37.30 \\
\hline
\end{tabular}

lasted 2 days. We assumed that therapists would have refresher training/mentoring for an average of 2 days a year, therefore we assumed that the costs of training would be repeated yearly. This seemed a reasonable approximation which accounts for the cost of continuous training for specialist groups, but excludes other types of training that may be unrelated to the CBA programme. ${ }^{139}$

Finally, therapists' travel expenses were computed from actual travel mileage declared by therapists and paid at $£ 0.40 /$ mile. Travel costs were allocated based on the actual travelled mileage for each group.

We did not incorporate a separate cost for space used within NHS physiotherapy departments. The space used for physiotherapy conducted on NHS premises (community and secondary care) comprises treatment cubicles, office space and a gym space with the space being used flexibly depending on the need for privacy, equipment, etc. Some patients may be treated in the gym and others in a treatment cubicle. We considered that the overheads in the Personal Social Services Research Unit costs would include the cost of accessing these spaces (on average). In the case of CBA, sessions were run for small groups (up to eight people) in spaces comparable to the gym or office used in normal NHS physiotherapy practice. From our experience, these two spaces are likely to be used for the majority of the patients, therefore we chose to use the estimated overheads of the Personal Social Services Research Unit as they incorporate space requirements. Although it could be argued that revising the cost of capital upwards to exclude the use of cubicles should be undertaken, we believe this is likely to have negligible impact on the cost of the CBA.
The total cost of one session was then obtained as a proportion (one-sixth) of the total cost of one CBA cycle, including supervision, training, consumables, equipment and travel time. The cost of one session was allocated to participants in proportion to session attendance, i.e. dividing the cost of one session by the number of participants in each group and session. Finally, the cost of CBA was computed for each participant multiplying the cost per patient of each session by the number of sessions attended.

The cost of the assessment visit was the cost of 1.5 hours' contact time at each therapist's hourly rate, and the cost of consumables and of CBA materials given to the participant (£2.60 in total per participant assessed). The cost of assessment was attributed to those participants who were assessed.

\section{The cost of active management}

At the start of the trial, all individuals were scheduled with a 15-minute nurse consultation at the time of recruitment and given The Back Book, a self-help advice booklet. The total cost of AM was $£ 14.05$ per participant. This included the salary cost of a practice nurse (£13.75), based on the cost per contact hour, ${ }^{134}$ and the duration of the consultation. The cost of The Back Book was $£ 1.00$ (assuming that the books are purchased in bulk quantities of 24 or more). The cost of AM was actualised to the year 2008 using the Office for National Statistics Retail Price Index. ${ }^{132}$

\section{Methods of analysis}

The base-case economic evaluation was conducted using the sample of participants who had 
completed questionnaires at baseline and at least at 3 months.

\section{Missing data}

For individuals who had missing cost data, unconditional mean imputation methods were used. ${ }^{140}$ For NHS costs, data were imputed only if some resource use items were present. In the case of private costs, cost data were also imputed for self-funded items. We did not undertake imputation if respondents failed to return the entire follow-up questionnaire.

Although unconditional mean imputation is recognised to underestimate the variability of estimates, we used this method because of simplicity and because the number of missing items was low for each resource consumption item.

Missing EQ-5D scores were estimated using multiple imputation techniques. ${ }^{141}$ Missing data were imputed only for those individuals who provided at least one item response for the EQ-5D and not for individuals where all items were missing. The cost-effectiveness incremental analysis was therefore conducted on the cases who had completed questionnaires at least at 3 months. This included the cases who had completed and returned a questionnaire with some missing values. For these cases, we then imputed the missing EQ-5D items. For the full economic evaluation, we used all the cases for which at least one measurement for costs was available, provided that the 3-month data were available.

\section{Total costs of care}

Total NHS costs for each participant were obtained by adding the cost of each health-care cost component described previously.

The total cost of care from the generic health-care viewpoint was calculated as the sum of the cost of private health care, transport and the cost of NHS care.

A descriptive analysis of resource use and the total annual cost of care was conducted using cases who provided complete follow-up at 12 months. Individuals who completed questionnaires at 6 or 12 months but not at 3 months were excluded from this analysis. Mean resource use and costs were tabulated by type of health-care services. Differences in mean costs between AM and $\mathrm{AM}+\mathrm{CBA}$ were tested using a $t$-test for differences in means. Standard errors for mean resource use were also reported. As the distribution of costs is positive and skewed, we constructed confidence intervals for mean costs using a bootstrap approach, sampling 1000 random samples of costs with samples of size equal to that of the original number of participants in each group. The $95 \%$ confidence limits were then calculated from the empirical distribution of the mean costs in each group and tabulated. We analysed differences in cost by sex, age, duration of LBP and RMQ scores using analysis of variance (ANOVA) for the total sample and both arms of the trial.

\section{Quality-adjusted life-years}

Utility weights were estimated transforming quality of life data (EQ-5D) into an index on the interval $[0,1]$, using an econometric model developed from time-trade-off health valuations provided by a sample of the general population in the UK. ${ }^{131,133}$ A utility weight represents the relative value to the individual of a certain health state, described by the combination of EQ-5D scores (see Chapter 3, Clinical outcomes, Secondary outcomes). The two extreme values of the interval indicate the utility weight for death and perfect health respectively. Compared with perfect health, a lower utility weight indicates a lower preference for less desirable health states.

Quality-adjusted life-years were then calculated for each participant as the 'area under the curve', the weighted sum of utility weights measured at baseline and 3, 6 and 12 months, and length of follow-up between each measurement, expressed in years. To compute the area under the curve, we assumed that utility between two periods was equal to the average of utility scores at two consecutive measurements.

We present a descriptive analysis of utility weights and total QALYs using participants who, at a minimum, provided at least some EQ-5D items and had completed follow-up at 3 months. Summary utility weights and QALYs were calculated at baseline, 3, 6 and 12 months, using $t$-tests for differences in means, and explored the variations in QALYs as a function of sex, age, duration of LBP and RMQ scores, using ANOVA.

We examined the association between costs or utility weight and commonly used prognostic factors, including age, sex and duration of back pain at entry, as these factors are easily identifiable 
and may offer useful indications for clinical practice.

Furthermore, we examined the impact of subdividing the study population into two groups, using a threshold RMQ score of $\geq 4$ versus $<4$. This threshold has been used by several large trials of LBP treatments as an entry criterion (excluding those with a score $<4$ ).

\section{Incremental analysis}

We calculated the ICUR as the ratio of the difference in mean costs of care (incremental costs) and of the difference in mean QALYs (incremental QALYs) between AM and AM+CBA.

The mean differences in costs and QALYs for $\mathrm{AM}+\mathrm{CBA}$ were estimated using a regression approach, including a treatment term in the regression for costs and QALYs, controlling for utility and other relevant baseline characteristics. Therefore the incremental costs and QALYs are the regression coefficient for the treatment term. A positive cost coefficient indicates a positive incremental cost associated with $\mathrm{AM}+\mathrm{CBA}$. Likewise, incremental QALYs indicate a positive or a negative gain in quality-adjusted survival. The ICUR is then the ratio of incremental costs and incremental QALYs, i.e. the ratio of the coefficients of the cost regression and the QALY regression respectively.

Mean costs and QALYs were estimated as the inverse probability weighted mean of the total quality-adjusted survival and cost of each participant in the two groups. ${ }^{140}$ The use of inverse probability weighting is justified because the rate of accrual of costs and QALYs varies across participants, although no deaths were observed during the study. Therefore the accrual of costs and QALYs may differ between patients who were followed up for the whole duration of the trial and those who were lost to follow-up at earlier dates.

Furthermore, a regression approach controls for factors that may be important in the prediction of costs and quality of life. The estimation of mean costs and QALYs should be adjusted a priori by utility at baseline. This is because the value of utility at baseline correlates with the total QALY as it enters its calculation directly. Any randomised comparison of two or more treatments is likely to show a difference in utility by group at baseline. Any imbalance in the utility at baseline, regardless of statistical significance, should always be regarded as a source of bias in the computation of the total QALY difference between the two groups. In addition, such analysis also allows for the incorporation of adjustments for control factors depending on the results of the cost and QALYs analysis, which may reveal additional significant predictors of costs and QALYs.

To investigate variations around the costutility ratio, we used non-parametric bootstrap techniques, sampling 1000 random samples of costs and QALYs with samples of size equal to that of the original number of participants in each group. The incremental costs and QALYs were then computed using the inverse-weighted regression approach for each sample.

The bootstrapping process consisted of two steps. First, a sample was drawn from each treatment group. These samples were sized proportionately to the randomised groups, and the bootstrap also randomly sampled baseline characteristics. The second step consisted of rerunning the original analysis on the bootstrapped data. This included recomputing the inverse probability weights and rerunning the regression on the samples generated by the bootstrap. Therefore the variation in the baseline characteristics is adjusted for in the regression and the variation in the costs and QALYs is summarised in the parameters of the regression coefficients.

Incremental costs and QALYs were plotted on the cost-effectiveness plane, the plot of the joint distribution of the values of incremental costs and incremental QALYs.

There are four potential combinations of incremental costs and QALYs:

- $\quad$ CBA is more effective and more costly than AM. This combination identifies points on quadrant I of the cost-effectiveness plane. In this case $\mathrm{AM}+\mathrm{CBA}$ would be considered costeffective if the incremental cost-utility ratio is lower than the societal decision-maker's willingness to pay. This is a monetary value that the decision-maker is willing to pay for one additional QALY, and as the decision-maker's willingness to pay threshold has proven empirically difficult to set, ${ }^{142}$ we calculated the cost-utility over the range $£ 0$ to $£ 35,000$. The decision-maker's cost-effectiveness threshold is indicated with the symbol $\lambda$.

- $\mathrm{AM}+\mathrm{CBA}$ is less costly and less effective than AM (points on quadrant III of the cost- 
effectiveness plane). Then CBA would be considered cost-effective if the ICUR is lower than the decision-maker's cost-effectiveness threshold.

- $\mathrm{CBA}$ is less expensive and more effective than AM (quadrant IV). Then CBA is 'dominant' because the decision-maker's choice would always be for CBA.

- $\mathrm{AM}+\mathrm{CBA}$ is more costly and less effective than AM alone (quadrant II). Then CBA is said to be 'dominated' by AM as there is no costeffectiveness threshold at which the decisionmaker would be willing to pay to implement CBA.

The cost-effectiveness plane allows visual assessment of the extent of cost-effectiveness or dominance combinations for the comparison of two interventions.

The cost-utility helps decision-making around the adoption of CBA. Because confidence intervals cannot be constructed for the ICUR, because of the presence of dominance combinations, we investigated the empirical probability that CBA is cost-effective using the net benefit statistic to estimate cost-effectiveness acceptability curves for CBA. ${ }^{143}$ The net benefit statistic,

Net benefit $=\lambda \times$ incremental

QALY - incremental cost

compares the monetary value of QALYs with the costs of the programme and is a summary indicator of the net contribution of CBA to health gain, expressed in monetary terms. Therefore $\mathrm{AM}+\mathrm{CBA}$ is cost-effective if net benefit is greater than zero, where $\lambda$ is the net health gain.

Net benefit was calculated for each bootstrap sample. Using the empirical frequency of positive net benefit we obtained the probability that $\mathrm{AM}+\mathrm{CBA}$ is cost-effective. The probability of CBA being cost-effective indicates the uncertainty around the cost-utility of CBA and therefore the degree of confidence associated with recommending the intervention. As the probability that CBA is cost-effective depends on the healthcare cost-effectiveness threshold, we plotted the probability that CBA is cost-effective as a function of $\lambda$ on the cost-effectiveness acceptability plot.
The results from the bootstrap process were similar to those of the regression model run on the original data set (what we would call the 'deterministic' analysis in other circumstances). The bootstrap allowed estimation of the variability of the incremental cost-effectiveness ratio (ICER) to construct cost-effectiveness acceptability curves. As a by-product of the bootstrap we used the bootstrap estimated for the regression coefficients to estimate their variability and distribution, although we report the exact estimates. This is because the inverse weight procedure works by subdividing costs over periods, therefore both the exact coefficients and the bootstrapped coefficients are derived from three separate regressions and the derivation of confidence intervals analytically is unyielding.

\section{Predictors of costs and utility and subgroup analysis}

The analysis of predictors was carried out using ANOVA controlled for treatment allocation. Although normally the stratification of costeffectiveness results should be driven by predictors of costs and quality of life, the extent to which predictors drive cost-effectiveness is generally unrelated to statistical significance. Therefore, to test the robustness of potential recommendations in favour of CBA, we conducted the cost-utility analysis separately for males and females, for individuals older than 60 or younger then 60 years and for individuals with RMQ scores of $<4$ or $\geq 4$. The latter analysis was also conducted to ensure the comparability of our results to results of other studies of LBP treatments. These findings are intended to assess the generalisability of the costeffectiveness analysis.

\section{Sensitivity analysis}

As there were cost outliers, we conducted a sensitivity analysis excluding the cases with the highest costs (above the 90th percentile of the distribution).

To assess the impact of inverse probability weights in the cost-effectiveness analysis we also conducted a sensitivity analysis including baseline adjustment and adjustment for predictors in the regression used to estimate the incremental costs and QALYs but not inverse weighting. 


\section{Results}

\section{Cases included in the economic evaluation}

The proportion of returned questionnaires valid for the economic evaluation at 3, 6 and 12 months of follow-up was between $70 \%$ and $77 \%$ (Figure 16).

The cost-utility analysis was conducted using the cases with complete follow-up at least for 3 months, $n=528$. This was the majority of cases in the trial $(75 \%)$. Individuals who completed questionnaires at 6 or 12 months but not at 3 months were excluded from the cost-effectiveness analysis.

For presentation purposes only, the descriptive analysis of mean resource use, costs and quality of life scores over a 1-year period was conducted on cases with complete follow-up at 12 months, $70 \%$ of the participants in the trial $(n=490)$.

There was no difference in length of follow-up between the AM+CBA and the AM groups for cases included in the economic evaluation. Mean follow-up for cases included was 360 days (SD 62 ) in the AM group and 365 days (SD 52) in the $\mathrm{AM}+\mathrm{CBA}$ group. Cases excluded had shorter follow-up: 85 days (SD 124) and 68 days (SD 88) in the $\mathrm{AM}$ and $\mathrm{AM}+\mathrm{CBA}$ groups respectively.

\section{Baseline characteristics of sample included and not included in the economic evaluation}

The majority of the sample included in the economic evaluation was female (59\% in both AM and $\mathrm{AM}+\mathrm{CBA}$ ), with equal age in the two groups (55 years on average), equal duration of LBP at recruitment (13 years) and equal proportion of individuals with RMQ scores $\geq 4$ (84\% in the AM group and $83 \%$ in the AM+CBA group). RMQ scores were significantly higher in women, with $88 \%$ of females having an RMQ score $\geq 4$, compared with $77 \%$ in men $(p=0.001)$.

The cases included in the economic analyses differed in some respects from the cases excluded (Table 39). In both groups, cases excluded were younger than cases included. There were also differences in the mean duration of LBT between cases included and excluded in the AM+CBA group, with lower duration of back pain in cases excluded from the economic evaluation. There were no differences in the severity of LBP between cases included and excluded (RMQ score $\geq 4: 84 \%$ in both groups). Mean utility score at baseline was with lower utility at entry for those excluded.

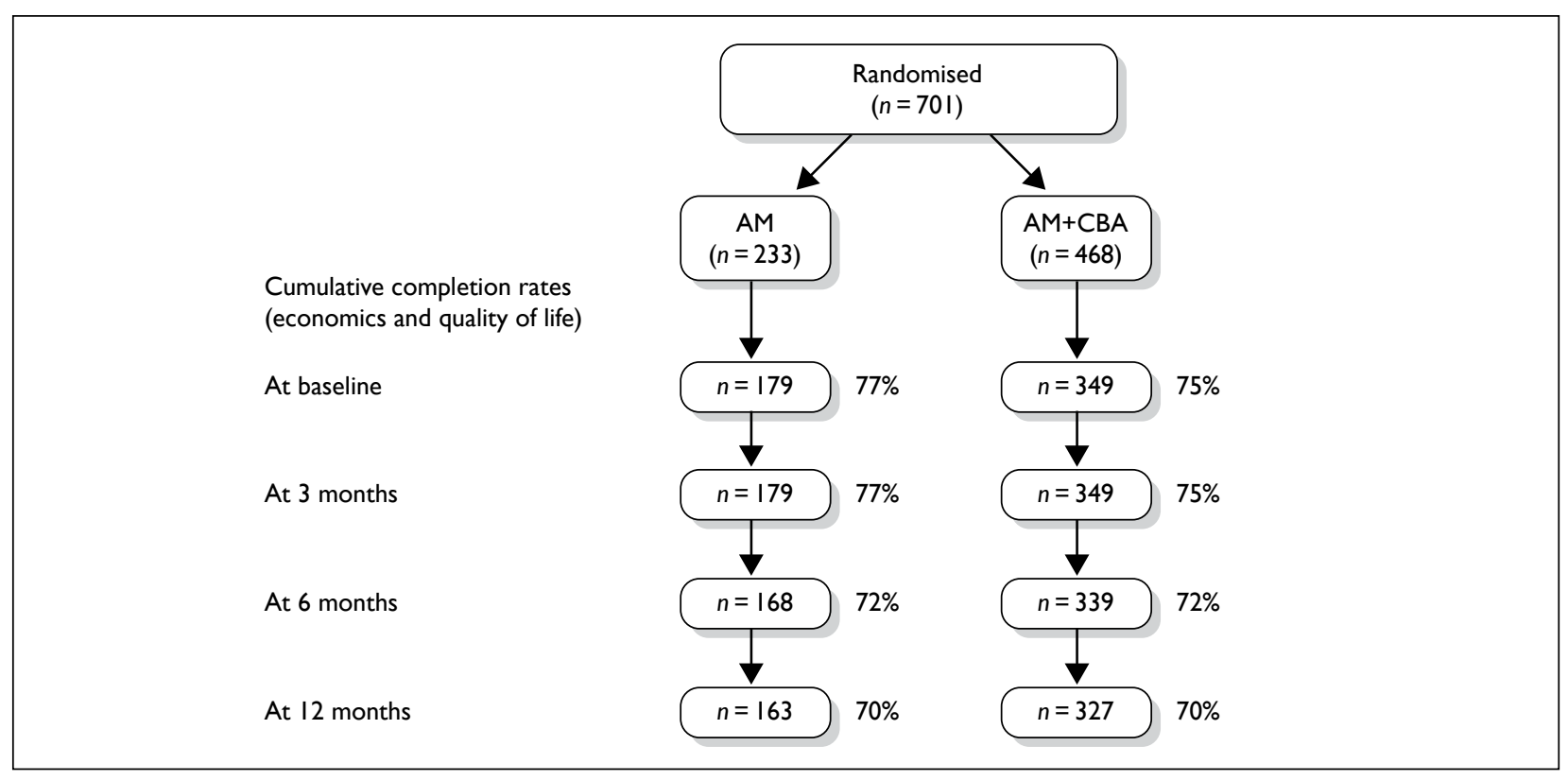

FIGURE 16 Flow chart of loss to follow-up - economic data. 


\section{Utility scores}

There were some differences in mean baseline utility score between AM (0.606) and the AM+CBA arm of the trial (0.574), although these differences were not statistically significant (data shown in Table 40). At baseline women expressed worse utility $(0.569)$ than men $(0.607)$, although differences were not statistically significant $(p=0.11)$. As would be expected, baseline utility differed according to baseline severity. Participants with RMQ scores $\geq 4$ or more had a mean baseline utility of 0.552 whereas those with RMQ scores $<4$ had a baseline utility of 0.750 (mean difference $0.198 ; p<0.001$ ).

Figure 17 shows the mean utility scores for $\mathrm{AM}+\mathrm{CBA}$ at baseline and follow-up time points. Utility scores improved during follow-up for the $\mathrm{AM}+\mathrm{CBA}$ group, with changes evident at 3 months and sustained to 12 months. By the end of followup, the mean utility scores had risen by 0.066 ,

TABLE 39 Baseline characteristics of cases included or excluded from economic evaluation

\begin{tabular}{|c|c|c|c|c|c|}
\hline \multirow[b]{2}{*}{$\begin{array}{l}\text { Characteristics at } \\
\text { baseline }\end{array}$} & \multicolumn{2}{|c|}{$\begin{array}{l}\text { Individuals included } \\
\text { (mean, standard error) }\end{array}$} & \multicolumn{2}{|c|}{$\begin{array}{l}\text { Individuals excluded } \\
\text { (mean, standard error) }\end{array}$} & \multirow[b]{2}{*}{$p$-value } \\
\hline & $A M(N=179)$ & $\begin{array}{l}A M+C B A \\
(N=349)\end{array}$ & $A M(N=54)$ & $\begin{array}{l}A M+C B A \\
(N=119)\end{array}$ & \\
\hline Age (years) & $54(I . I)$ & $55(0.8)$ & $50(2.2)$ & $50(1.4)$ & 0.001 \\
\hline$\%$ female & $59 \%(3.6 \%)$ & $59 \%(2.6 \%)$ & $63 \%(6.6 \%)$ & $57 \%(4.6 \%)$ & 0.910 \\
\hline Duration of LBP (years) & $13(0.9)$ & $13(0.9)$ & $12(2.0)$ & $9(1.4)$ & 0.030 \\
\hline Utility scores & $0.606(0.019)$ & $0.574(0.014)$ & $0.481(0.045)$ & $0.525(0.026)$ & 0.002 \\
\hline
\end{tabular}

TABLE 40 Mean utility scores at baseline, 3, 6 and 12 months

\begin{tabular}{|c|c|c|c|c|c|}
\hline & \multicolumn{2}{|l|}{$\mathbf{A M}^{\mathrm{a}}$} & \multicolumn{2}{|c|}{$A M+C B A^{a}$} & \multirow[b]{2}{*}{ Mean difference } \\
\hline & Mean & SE & Mean & SE & \\
\hline Baseline $(n=528)$ & 0.606 & 0.019 & 0.574 & 0.014 & -0.032 \\
\hline 3 months $(n=528)$ & 0.567 & 0.022 & 0.628 & 0.014 & 0.061 \\
\hline 6 months $(n=527)$ & 0.593 & 0.021 & 0.630 & 0.016 & 0.037 \\
\hline 12 months $(n=490)$ & 0.592 & 0.023 & 0.640 & 0.016 & 0.048 \\
\hline
\end{tabular}

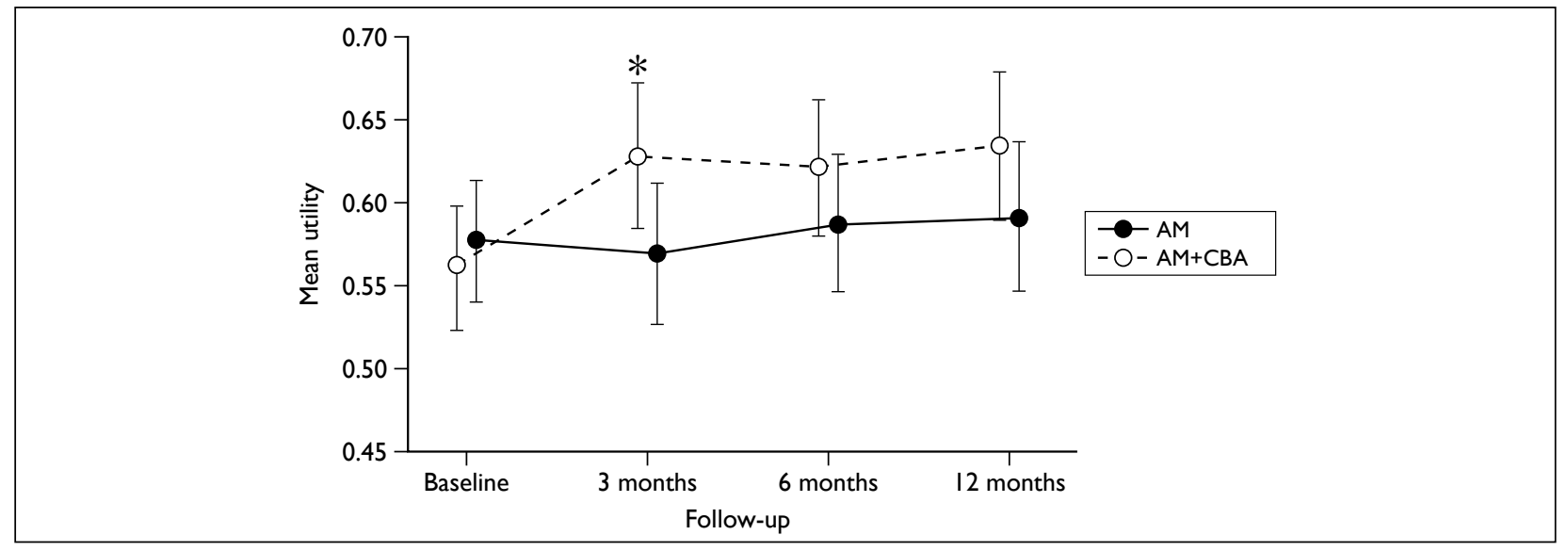

FIGURE I7 Mean utility scores at baseline, 3, 6 and 12 months. Vertical bars show $95 \%$ confidence intervals; data points are offset to improve visibility. *Statistically significant difference between $A M$ and $A M+C B A, p=0.001$. 
whereas in the AM group mean utility dropped initially at 3 months but improved in the follow-up, and remained unchanged over the 12 months, with a drop of -0.014 at 12 months in comparison with baseline.

\section{Quality-adjusted life-years}

We calculated the QALYs as the weighted mean of utility weights between two measurements and time spent in the study. Figure 18 gives the cumulative QALYs over 3, 6 and 12 months. These are the total QALYs from baseline up to each time point. AM+CBA accumulated 0.662 mean QALYs and AM accumulated 0.624 mean QALYs over the 12-month follow-up, with an unadjusted difference of 0.038 QALYs at 12 months (Figure 18). These are crude estimates and are the mean of the area under the curve, obtained from the crude QALY scores multiplied by the length of followup. These estimates are illustrated here only for reference because they are not the final estimates of incremental QALYs based on the regression approach used to estimate the cost-effectiveness of CBA.

\section{Incremental quality-adjusted life-years}

The adjusted estimate of the incremental QALYs used in the cost-effectiveness analysis was obtained by fitting an ordinary least squares regression to the cumulative QALYs and adjusting for utility scores at baseline and for sex, and weighted by the inverse probability of follow-up. This section details the results of the regression model adjusted for the predictors for QALYs, first without and then including the inverse probability weighting.

The adjusted regression equation included a term for baseline utility scores and two dummy terms for female gender and treatment.

Regression coefficients were then estimated using the inverse probability weighting method described in Tables 41 and 42.

This regression was weighted by the inverse probability of follow-up. With this approach, the mean total QALY with AM was 0.603 and the incremental QALY associated with treatment with $\mathrm{AM}+\mathrm{CBA}$ was +0.099 .

\section{NHS resource consumption}

A large proportion of trial participants, $44 \%$, had no contacts with primary-care services during the study, with the exception of the CBA programme. The most frequently used NHS services were GP and physiotherapy consultations (Table 43). GPs were consulted by $50 \%$ of the trial participants, with $17 \%$ of the trial participants consulting more than three times over the course of follow-up. Physiotherapy was used by $19 \%$ of participants. Two-thirds of trial participants who were seen by a GP were prescribed pharmacological agents. Fifty per cent of the sample received more than three prescriptions over the follow-up period. The use of diagnostics tests was limited, as was use of hospital care. There were 11 admissions overall: five in the

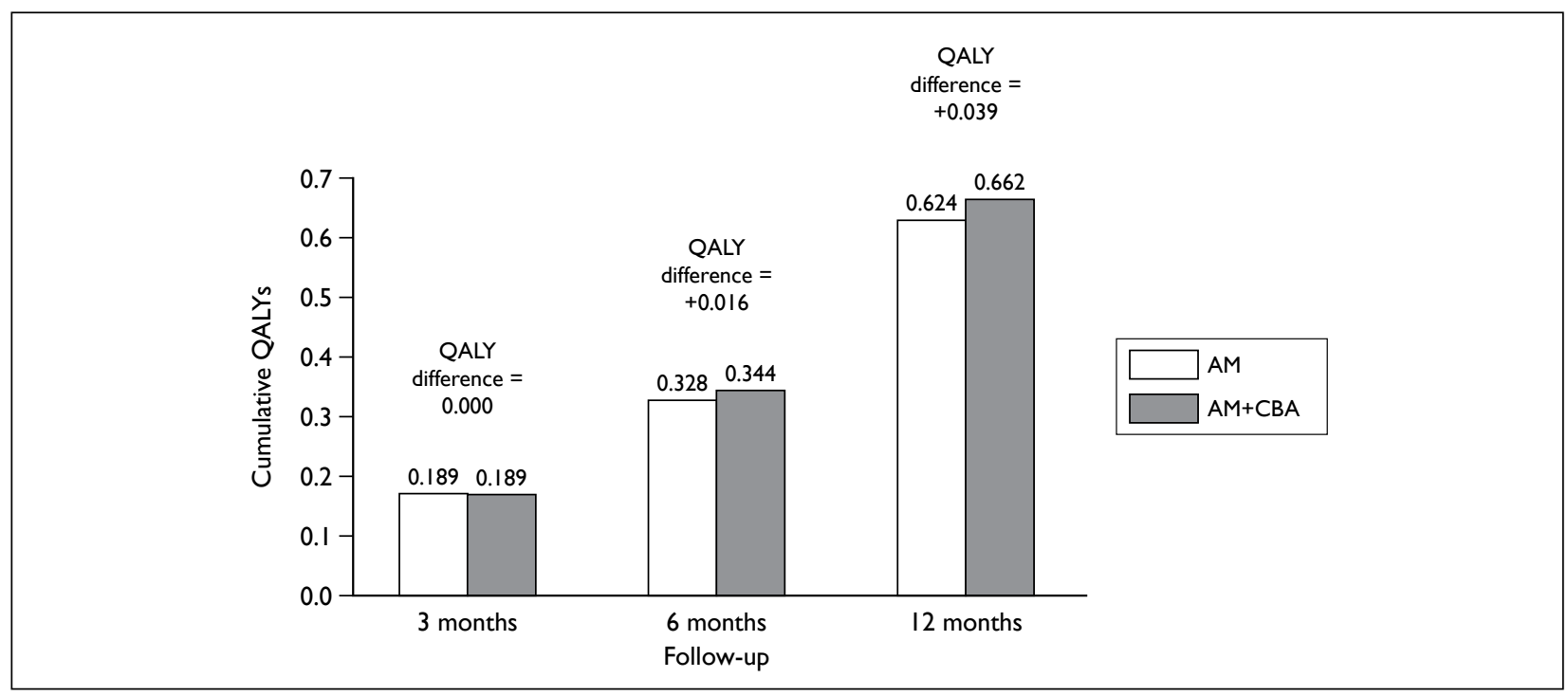

FIGURE 18 Cumulative quality-adjusted life-years (QALYS) - unadjusted. 
TABLE 4 I Regression coefficients, QALYs, unadjusted by inverse probability weighting

$\begin{array}{lclll} & \text { Coefficient } & \text { SE } & \mathbf{p}>\mathbf{t} & \mathbf{9 5 \%} \mathbf{C l} \\ \text { Gender } & -0.02 & 0.02 & 0.333 & -0.05 \text { to } 0.02 \\ \text { Utility at baseline } & 0.66 & 0.03 & 0.000 & 0.60 \text { to } 0.72 \\ \text { Treatment (CBA) } & 0.06 & 0.02 & 0.000 & 0.03 \text { to } 0.10 \\ \text { Constant } & 0.21 & 0.03 & 0.000 & 0.16 \text { to } 0.26 \\ \text { SE, standard error. } & & & & \end{array}$

TABLE 42 Regression coefficients, QALYs, adjusted by inverse probability weighting

$\begin{array}{lclll} & \text { Coefficient } & \text { SE } & \mathbf{p}>\mathbf{t} & \mathbf{9 5 \%} \mathbf{C l} \\ \text { Gender } & -0.04 & 0.02 & 0.047 & -0.09 \text { to } 0.01 \\ \text { Utility at baseline } & 0.64 & 0.05 & 0.000 & 0.54 \text { to } 0.73 \\ \text { Treatment (CBA) } & 0.10 & 0.03 & 0.000 & 0.04 \text { to } 0.16 \\ \text { Constant } & 0.25 & 0.04 & 0.000 & 0.18 \text { to } 0.33 \\ \text { SE, standard error. } & & & & \end{array}$

TABLE 43 Mean resource consumption, NHS, by type

\begin{tabular}{|c|c|c|c|c|c|}
\hline \multirow[b]{2}{*}{ Service } & \multirow[b]{2}{*}{ Item } & \multicolumn{2}{|c|}{$A M(N=163)$} & \multicolumn{2}{|c|}{$A M+C B A(N=327)$} \\
\hline & & Mean no. & SE & Mean no. & SE \\
\hline \multirow[t]{3}{*}{ Primary-care consultations } & General practitioner & 1.86 & 0.017 & 2.06 & 0.011 \\
\hline & Practice nurse & 0.11 & 0.003 & 0.16 & 0.003 \\
\hline & Physiotherapist & 0.90 & 0.016 & 0.91 & 0.008 \\
\hline \multirow[t]{4}{*}{ Hospital care } & A\&E visits & 0.02 & 0.001 & 0.03 & 0.001 \\
\hline & Outpatient & 0.21 & 0.004 & 0.29 & 0.003 \\
\hline & Psychologist & 0.09 & 0.004 & 0.10 & 0.002 \\
\hline & Admissions & 0.03 & 0.000 & 0.02 & 0.000 \\
\hline \multirow[t]{4}{*}{ Diagnostic tests } & X-ray & 0.28 & 0.005 & 0.17 & 0.002 \\
\hline & CT scan & 0.01 & 0.001 & 0.03 & 0.001 \\
\hline & MRI scan & 0.04 & 0.001 & 0.10 & 0.001 \\
\hline & Blood tests & 0.50 & 0.007 & 0.63 & 0.007 \\
\hline \multirow[t]{5}{*}{ Drug prescriptions } & Pain killers & 2.22 & 0.028 & 2.01 & 0.010 \\
\hline & Anti-inflammatory drugs & 2.02 & 0.079 & 1.09 & 0.007 \\
\hline & Gels and creams & 0.24 & 0.006 & 0.20 & 0.003 \\
\hline & Sleeping pills & 0.48 & 0.018 & 0.28 & 0.004 \\
\hline & Antidepressants & 0.37 & 0.007 & 0.40 & 0.004 \\
\hline
\end{tabular}

$\mathrm{AM}$ and six in the AM+CBA group. Six admissions were day cases, three in each group.

Overall there was no difference in the mean number of each type of service accessed in the two groups. None of the differences in NHS resource use was statistically significant. 
TABLE 44 Mean resource consumption, private health-care expenditure, by type

\begin{tabular}{|c|c|c|c|c|c|}
\hline \multirow[b]{2}{*}{ Service } & \multirow[b]{2}{*}{ Item } & \multicolumn{2}{|c|}{$A M(N=163)$} & \multicolumn{2}{|c|}{$A M+C B A(N=327)$} \\
\hline & & Mean no. & SE & Mean no. & SE \\
\hline \multirow{8}{*}{$\begin{array}{l}\text { Primary-care and } \\
\text { community-care } \\
\text { consultations }\end{array}$} & Physiotherapy & 0.32 & 0.009 & 0.40 & 0.007 \\
\hline & Osteopath & 0.53 & 0.014 & 0.28 & 0.006 \\
\hline & Chiropractor & 0.45 & 0.017 & 0.83 & 0.020 \\
\hline & Psychologist & - & - & 0.03 & 0.002 \\
\hline & Counselling & - & - & 0.01 & 0.001 \\
\hline & Massage therapy & 0.40 & 0.013 & 0.47 & 0.007 \\
\hline & Aromatherapy & - & - & 0.09 & 0.003 \\
\hline & Acupuncture & 0.09 & 0.005 & 0.22 & 0.004 \\
\hline \multirow[t]{6}{*}{ Equipment } & Exercise equipment & 0.24 & 0.004 & 0.20 & 0.002 \\
\hline & Back support items & 0.16 & 0.003 & 0.13 & 0.001 \\
\hline & Aids & 0.03 & 0.001 & 0.02 & 0.001 \\
\hline & Small house furniture & 0.07 & 0.002 & 0.05 & 0.001 \\
\hline & Beds and mattresses & 0.22 & 0.003 & 0.24 & 0.002 \\
\hline & Other equipment & 0.07 & 0.002 & 0.07 & 0.001 \\
\hline \multirow[t]{2}{*}{ Hospital care } & Outpatient & 0.06 & 0.002 & 0.08 & 0.002 \\
\hline & Admissions & 0.01 & 0.000 & 0.01 & 0.000 \\
\hline \multirow[t]{5}{*}{ Diagnostic tests } & X-ray & 0.01 & 0.001 & 0.04 & 0.001 \\
\hline & CT scan & - & - & 0.01 & 0.000 \\
\hline & MRI scan & 0.01 & 0.001 & 0.02 & 0.000 \\
\hline & Blood tests & 0.01 & 0.001 & 0.09 & 0.002 \\
\hline & Other tests & 0.01 & 0.000 & - & - \\
\hline \multirow[t]{4}{*}{ Drug purchases } & Pain killers & 4.99 & 0.065 & 3.65 & 0.024 \\
\hline & Anti-inflammatory drugs & 2.94 & 0.049 & 1.77 & 0.012 \\
\hline & Gels and creams & 0.88 & 0.012 & 0.72 & 0.006 \\
\hline & Other drugs & 0.27 & 0.005 & 0.28 & 0.004 \\
\hline
\end{tabular}

\section{Private resource consumption}

There were no differences in the use of privately paid health-care services by treatment (Table 44). Overall, 23\% of participants reported using private health-care services, and $16 \%$ of all participants accessed these services more than four times. A substantial proportion of participants $(63 \%)$ opted to purchase medications over the counter. The number of over the counter purchases was greater than the number of NHS prescriptions, with over two-thirds of those individuals purchasing over the counter medications reporting purchasing four or more items.

Access to primary and hospital care was mainly through NHS services, whereas equipment, aids and adaptations were entirely paid for privately.
Purchases of equipment, aids and adaptations were reported by $36 \%$ of participants.

\section{Costs \\ The within-trial cost of the $A M+C B A$ programme}

The AM+CBA programme was administered to 62 groups attended by 7.6 participants (minimum four, maximum 12) on average, and run by 19 therapists. Overall the programme delivered 372 sessions, with a total number of attendants equal to 161. For each group, there were 9 hours of contact time, and 8.4 hours on average for non-contact time, including 6.15 hours' support time (minimum 1, maximum 11.5 hours) and 2.25 hours' travel time (minimum 0 , maximum 14 hours). Therefore the mean contact to non- 
contact time ratio was $1: 1.9$. The mean salary cost of 1 contact hour (including non-contact time) was $£ 33.65$.

The mean total cost per group of the CBA programme was $£ 826.08$ (minimum $£ 584.09$, maximum £1294.35). This amount comprised $£ 583.38$ for salary costs, $£ 84.21$ for supervision costs, $£ 39.50$ for disposables and equipment, $£ 17.30$ for travel costs (40 miles on average per group) and $£ 101.69$ for training costs. Assessment cost was $£ 53.45$ including consumables and patient information material, and the cost of the AM advice at recruitment was $£ 16.32$ (2008 prices). The mean cost per patient for attending a cycle of CBA was $£ 187$ (SE 0.266).

\section{Total health-care costs - NHS perspective}

Total NHS costs were approximately $£ 247$ for the AM group and $£ 421$ for the CBA group. The difference was almost entirely attributable to the cost of CBA, $£ 187$, with the cost of NHS care other than attending the CBA programme being very similar for both groups. Mean health-care costs, excluding AM and CBA, were approximately $£ 207$ for the AM and $£ 217$ for the CBA group. The greatest cost was for consultations in primary care (GPs and physiotherapists) and for outpatient consultations and hospital admissions. In the trial, one patient initially allocated to AM received $\mathrm{AM}+\mathrm{CBA}$ instead, at a cost of $£ 179$.

Table 45 presents mean programme costs for individuals with complete data at 12 months.

None of the cost differences were statistically significant with the exception of other consultations $(p=0.045)$, CBA costs and as a consequence of the latter, total costs.

TABLE 45 Mean costs of care $(£)$, NHS

\begin{tabular}{|c|c|c|c|c|c|}
\hline \multirow[b]{2}{*}{ Service } & \multirow[b]{2}{*}{ Item } & \multicolumn{2}{|l|}{$A M(N=163)$} & \multicolumn{2}{|c|}{$A M+C B A(N=327)$} \\
\hline & & Mean cost $(t)$ & $\begin{array}{l}95 \% \mathrm{Cl} \\
\text { (bootstrap) }\end{array}$ & Mean cost $(t)$ & $\begin{array}{l}96 \% \mathrm{Cl} \\
\text { (bootstrap) }\end{array}$ \\
\hline \multirow{4}{*}{$\begin{array}{l}\text { Primary-care } \\
\text { consultations }\end{array}$} & General practitioner & 63.20 & 49.85 to 78.95 & 69.96 & 57.27 to 84.74 \\
\hline & Practice nurse & 0.99 & 0.44 to 1.66 & 1.46 & 0.74 to 2.45 \\
\hline & Physiotherapist & 28.55 & 16.35 to 41.94 & 28.89 & 19.63 to 39.67 \\
\hline & Other consultations & 2.43 & 0.00 to 6.107 & 0.00 & 0.00 to 0.00 \\
\hline \multirow[t]{4}{*}{ Hospital care } & A\&E visits & 4.62 & 0.00 to 11.55 & 5.76 & 1.15 to 11.79 \\
\hline & Outpatient & 24.19 & 13.82 to 37.67 & 33.08 & 22.40 to 45.83 \\
\hline & Psychologist & 5.75 & 0.41 to 13.15 & 6.67 & 2.37 to 12.76 \\
\hline & Admissions & 22.44 & 7.19 to 41.42 & 11.18 & 3.58 to 20.57 \\
\hline \multirow[t]{5}{*}{ Diagnostic tests } & X-ray & 8.73 & 5.32 to 12.72 & 5.42 & 3.54 to 7.65 \\
\hline & CT scan & 1.60 & 0.00 to 4.01 & 3.99 & 1.59 to 6.78 \\
\hline & MRI scan & 9.88 & 2.78 to 19.07 & 22.95 & 13.79 to 34.03 \\
\hline & Blood tests & 2.15 & I. 46 to 3.03 & 2.15 & 1.45 to 3.12 \\
\hline & Other diagnostics & 0.00 & - & 1.04 & 0.26 to 2.34 \\
\hline \multirow{6}{*}{$\begin{array}{l}\text { Drug } \\
\text { prescriptions }\end{array}$} & Pain killers & 10.27 & 7.43 to $|3.7|$ & 9.30 & 7.80 to 11.16 \\
\hline & Anti-inflammatory drugs & 16.77 & 6.46 to 36.61 & 9.03 & 7.15 to 11.27 \\
\hline & Gels and creams & 1.41 & 0.65 to 2.43 & 1.19 & 0.68 to 1.82 \\
\hline & Sleeping pills & 1.78 & 0.38 to 3.61 & 1.04 & 0.52 to 1.66 \\
\hline & Antidepressants & 2.18 & 1.15 to 3.51 & 2.35 & 1.55 to 3.29 \\
\hline & Other drugs & 0.86 & 0.23 to 1.83 & 1.50 & 0.22 to 3.47 \\
\hline \multicolumn{2}{|c|}{ All NHS other care (mean) } & 207.23 & 160.09 to 262.90 & 217.53 & 178.75 to 262.21 \\
\hline & AM & 16.32 & - & 16.32 & - \\
\hline & CBA & $1.10^{\mathrm{a}}$ & 0.00 to 3.31 & 187.67 & 178.45 to 196.80 \\
\hline \multicolumn{2}{|c|}{ Overall mean NHS costs } & 224.65 & 176.99 to 275.66 & 421.52 & 378.11 to 468.70 \\
\hline
\end{tabular}


From the descriptive analysis in the previous section, there is no apparent strong predictor of differences in accrual of total costs and QALYs by group with the exception of RMQ scores. There were no differences in the mean cost of the CBA programme by age, sex, severity or duration of low back pain.

Total cost of NHS care was predicted by the RMQ score and, to a lesser extent, by sex. An RMQ score of $\geq 4$ was associated with a statistically significant increase in mean expenditure of $£ 185.42$. This difference was attributable to NHS care other than the CBA programme. Individuals with higher scores spent more in GP consultations (on average, $+£ 54.37, p=0.000)$, physiotherapy $(+£ 25.58$, $p=0.012)$, hospitalisations $(+£ 31.49, p=0.005)$, painkillers $(+£ 4.83, p=0.019)$, antidepressants $(+£ 2.02, p=0.027)$, and diagnostic tests such as MRI $(+£ 22.44, p=0.027)$ and X-ray $(+£ 6.53$, $p=0.007)$.

Other health-care costs tended to be larger for women than for men in both treatment groups, although not statistically significantly so. The cost difference by sex was $£ 64.70(p=0.098)$. Women tended to have higher costs for some NHS health-care items, albeit this difference was often more marked in the AM group. There were sex differences in the cost generated from drug therapies (sleeping pills, $-£ 2.90, p=0.09$ ), use of X-rays $(+£ 4, p=0.021)$ and hospital admissions $(+£ 20, p=0.024)$. There were no sizeable differences in costs by age. The cost of care increased with age by a factor of $£ 1.13$ per year of age $(p=0.4)$.

The duration of LBP did not have an impact on the cost of care.

\section{Total health-care costs, general health- care perspective}

There was no significant difference in private costs between $\mathrm{AM}$ and $\mathrm{AM}+\mathrm{CBA}$ although there was a trend toward higher private costs in the AM $+\mathrm{CBA}$ arm [by approximately $+£ 130$ on average (Table 46)]. The majority of private costs were generated by equipment and house adaptations, which cost $£ 135$ and $£ 163$ in total in the AM and $\mathrm{AM}+\mathrm{CBA}$ group respectively, and consultations with medical and health-care professionals. The costs of these consultations were $£ 65$ for AM and $£ 82$ for AM+CBA. Overall, hospital admissions were low, but there were more admissions in the $\mathrm{AM}+\mathrm{CBA}$ arm, and as these are expensive resources, the costs associated with hospitalisation were also higher in the AM+CBA group.

Private health-care costs were higher in participants with a baseline score $\geq 4$ on the RMQ $(+£ 197.68, p=0.09)$. This difference, although not statistically significant, is likely to assume economic significance. There were no particular services that explained this increased expenditure, apart from travel costs $(+£ 13.90$, $p=0.01$ ). Private expenditure was weakly associated with sex, with higher average costs for women than men, $+£ 90(p=0.38)$. The impact of age was larger for private costs compared with NHS costs. Expenditure on chiropractic increased with age $(+£ 1.18$ per additional year of patient age; $p=0.068$ ), acupuncture costs increased by a factor of $+£ 0.35(p=0.057)$ and cost of aids increased by $+£ 0.28(p=0.037)$. However, these effects were not economically significant given the extent of the total expenditure. The duration of LBP also had no impact on costs with only negligible differences in expenditure per year of LBP.

Overall, when broader health-care costs were considered, the total cost of AM+CBA was just under twice that of AM only. This difference arose from two sources - private expenditure and the additional costs of the AM+CBA programme (Table 47).

\section{Incremental costs}

The adjusted estimate of the incremental costs used in the cost-effectiveness analysis was obtained by fitting an ordinary least squares regression to the cumulative cost of care, using a regression model similar to that reported for QALYs (see Incremental QALYs).

The regression model included adjustments for utility scores at baseline and for sex, and was weighted by the inverse probability of follow-up. The results of the regression model adjusted for the predictors for costs, first without and then including the inverse probability weighting, are reported in Tables 48 and 49. The adjusted regression equation included a term for baseline utility scores and two dummy terms for female gender and treatment. These tables report the exact coefficient estimates, rather than expected value obtained from the bootstrap procedure.

Regression coefficients were then estimated using the inverse probability weighting method. 
TABLE 46 Mean costs $(E)$ of private health care and related costs

\begin{tabular}{|c|c|c|c|c|c|}
\hline \multirow[b]{2}{*}{ Service } & \multirow[b]{2}{*}{ Item } & \multicolumn{2}{|l|}{$A M(N=163)$} & \multicolumn{2}{|c|}{$A M+C B A(N=327)$} \\
\hline & & Mean cost $(t)$ & $\begin{array}{l}95 \% \mathrm{Cl} \\
\text { (bootstrap) }\end{array}$ & Mean cost $(t)$ & $\begin{array}{l}95 \% \mathrm{Cl} \\
\text { (bootstrap) }\end{array}$ \\
\hline \multirow{9}{*}{$\begin{array}{l}\text { Primary-care } \\
\text { consultations }\end{array}$} & Physiotherapy & 12.19 & 4.45 to 21.56 & 15.42 & 7.13 to 25.93 \\
\hline & Osteopath & 23.64 & 10.33 to 40.09 & 12.19 & 4.61 to 22.90 \\
\hline & Chiropractor & 15.81 & 3.90 to 34.22 & 29.47 & 10.20 to 60.13 \\
\hline & Psychologist & 0.00 & - & 2.37 & 0.00 to 7.10 \\
\hline & Counselling & 0.00 & - & 0.61 & 0.00 to 1.83 \\
\hline & Massage therapy & 10.04 & 3.04 to 18.33 & 11.76 & 6.26 to 18.16 \\
\hline & Aromatherapy & 0.00 & - & 2.17 & 0.23 to 5.11 \\
\hline & Acupuncture & 3.17 & 0.00 to 8.25 & 7.49 & 3.11 to 13.08 \\
\hline & All primary care (mean) & 64.85 & & 81.48 & \\
\hline \multirow[t]{7}{*}{ Equipment } & Equipment (TENS) & 6.80 & 3.65 to 10.43 & 9.30 & 4.95 to 15.75 \\
\hline & Back support items & 4.29 & 1.97 to 7.11 & 4.28 & 2.45 to 6.66 \\
\hline & Aids & 1.12 & 0.06 to 2.72 & 3.30 & 0.21 to 7.47 \\
\hline & Small house furniture & 23.17 & $4.4 I$ to 48.70 & 28.95 & 12.09 to 48.55 \\
\hline & Beds and mattresses & 96.60 & 60.22 to 137.49 & 113.64 & 75.90 to 158.33 \\
\hline & Other equipment & 2.53 & 0.78 to 4.67 & 3.79 & I.18 to 7.88 \\
\hline & All equipment (mean) & $134.5 \mid$ & & 163.26 & \\
\hline \multirow[t]{4}{*}{ Hospital care } & Outpatient consultations & II.20 & 2.24 to 22.39 & 15.07 & 6.14 to 26.79 \\
\hline & Admissions, paid by patient & 3.53 & 0.00 to 10.58 & 56.04 & 0.00 to 146.94 \\
\hline & $\begin{array}{l}\text { Admissions, paid by health } \\
\text { insurance }\end{array}$ & 3.53 & 0.00 to 10.58 & 2.91 & 0.00 to 8.72 \\
\hline & All hospital care (mean) & 18.26 & & 74.02 & \\
\hline \multirow[t]{6}{*}{ Diagnostic tests } & X-ray & 1.06 & 0.00 to 3.17 & 3.69 & 1.05 to 7.64 \\
\hline & CT scan & 0.00 & - & 3.37 & 0.00 to 8.43 \\
\hline & MRI scan & 7.34 & 0.00 to 18.34 & 9.14 & 1.83 to 18.28 \\
\hline & Blood tests & 1.35 & 0.00 to 3.37 & 9.74 & 4.03 to 16.79 \\
\hline & Other & 0.52 & 0.00 to 1.83 & 0.00 & 0.00 to 0.00 \\
\hline & All diagnostics (mean) & 10.27 & & 25.94 & \\
\hline \multirow{5}{*}{$\begin{array}{l}\text { Drug } \\
\text { prescriptions }\end{array}$} & Painkillers & 6.50 & 4.48 to 9.08 & 6.87 & 4.87 to 9.18 \\
\hline & Anti-inflammatory & 4.99 & 3.09 to 7.18 & 9.22 & 4.90 to 14.84 \\
\hline & Topical gels and creams & 4.01 & 2.32 to 6.05 & 7.91 & 2.65 to 15.59 \\
\hline & Other drugs & 2.76 & 1.35 to 4.49 & 2.69 & 1.52 to 4.17 \\
\hline & Overall drugs (mean) & 18.26 & & 26.69 & \\
\hline \multicolumn{2}{|l|}{ Transport costs } & 24.50 & 19.53 to 30.05 & 29.17 & 23.70 to 35.14 \\
\hline \multicolumn{2}{|c|}{$\begin{array}{l}\text { Overall mean cost of private health care and } \\
\text { related costs }\end{array}$} & 270.65 & 205.87 to 350.63 & 400.55 & 292.16 to 541.27 \\
\hline
\end{tabular}

incremental cost of treatment with $\mathrm{AM}+\mathrm{CBA}$ was $+£ 181.55$.

The cost coefficients calculated from the healthcare perspective are reported in Table 49.
The inverse weighting had no impact on the regression as the coefficients were almost identical to the unadjusted analysis. With this approach, the mean total cost with AM was $£ 224.94$ and the 
TABLE 47 Mean and total NHS and private health-care costs $(£)$ - NHS and general health-care perspectives

\begin{tabular}{|c|c|c|c|c|}
\hline \multirow[b]{2}{*}{ Service } & \multicolumn{2}{|l|}{ AM } & \multicolumn{2}{|l|}{$A M+C B A$} \\
\hline & Mean cost $(t)$ & 95\% Cl (bootstrap) & Mean cost $(t)$ & 95\% Cl (bootstrap) \\
\hline $\begin{array}{l}\text { Total cost initial intervention (AM, } \\
A M+C B A)(a)\end{array}$ & 17.42 & & 203.99 & \\
\hline Total other NHS costs (b) & 207.23 & 160.09 to 262.90 & 217.53 & 178.75 to 262.21 \\
\hline Total NHS costs $(c=a+b)$ & 224.65 & 176.99 to 275.66 & 421.52 & 378.11 to 468.70 \\
\hline Total cost of private health care (d) & 270.65 & 205.87 to 350.63 & 400.55 & 292.16 to 541.27 \\
\hline $\begin{array}{l}\text { Total costs, general health-care } \\
\text { perspective }(e=c+d)\end{array}$ & 493.68 & 405.08 to 590.37 & 821.71 & 695.11 to 964.48 \\
\hline
\end{tabular}

TABLE 48 Regression coefficients, QALYs, unadjusted by inverse probability weighting - NHS perspective

\begin{tabular}{lcccc} 
& Coefficient $(\boldsymbol{\epsilon})$ & $\mathbf{S E}(\boldsymbol{\epsilon})$ & $\mathbf{p}>\boldsymbol{t}$ & $\mathbf{9 5 \%} \mathbf{C I}(\boldsymbol{\epsilon})$ \\
\hline Gender & 32.72 & 33.98 & 0.000 & 113.69 to 247.22 \\
Utility at baseline & -365.90 & 32.79 & 0.319 & -31.70 to 97.14 \\
Treatment (CBA) & 180.46 & 60.77 & 0.000 & -485.29 to -246.51 \\
Constant & 419.48 & 51.03 & 0.000 & 319.23 to 519.72 \\
SE, standard error. & & & &
\end{tabular}

TABLE 49 Regression coefficients, QALYs, adjusted by inverse probability weighting - general health-care perspective

\begin{tabular}{lcccc} 
& Coefficient $(\boldsymbol{\epsilon})$ & $\mathbf{S E}(\boldsymbol{\epsilon})$ & $\boldsymbol{p}>\boldsymbol{t}$ & $\mathbf{9 5 \%} \mathbf{C l}(\boldsymbol{\epsilon})$ \\
\hline Gender & 56.88 & $102.4 \mathrm{I}$ & 0.002 & 113.85 to 516.23 \\
Utility at baseline & -590.10 & 98.83 & 0.565 & -137.26 to 251.03 \\
Treatment (CBA) & 315.03 & 183.15 & 0.001 & -949.91 to -230.30 \\
Constant & 797.04 & 153.78 & 0.000 & 494.95 to 1099.14 \\
SE, standard error. & & & &
\end{tabular}

\section{Cost-effectiveness}

Separate models are presented for the NHS and the general health-care perspectives. Expected incremental costs and incremental QALYs are reported in Tables 50 and 51.

\section{NHS perspective}

From the NHS perspective, CBA was associated with an increase in health-care costs, $+£ 178.06$, and an improvement in QALYs, +0.099 . The ICUR was $£ 1786$, indicating that CBA is costeffective at the currently accepted decision-maker's cost-effectiveness threshold.

The cost-utility was not sensitive to cost outliers. The ICUR obtained excluding the cases with costs higher than the 90th percentile was $£ 1797$, with an incremental cost of $£ 162.44$.

The cost-utility was sensitive to the inverse weighting method. Excluding inverse weighting, the model estimated +0.061 incremental QALYs and $+£ 177.43$ incremental costs (from the bootstrap procedure), yielding an ICUR of £2909. This ICUR remains nevertheless well below the recognised decision-maker's cost-effectiveness threshold.

\section{Uncertainty}

To assess the variability of this estimate, we plotted the incremental costs and incremental QALYs on the cost-effectiveness plane (Figure 19) calculated for each of the 1000 bootstrap estimates upon 
TABLE 50 Incremental costs and $Q A L Y s, A M+C B A$ compared with $A M-N H S$ perspective

\begin{tabular}{llll} 
& AM & AM+CBA & Difference \\
\hline Total NHS costs & $£ 278.99$ & $£ 457.05$ & $+£ 178.06$ \\
Total QALYs & 0.604 & 0.703 & +0.099 \\
ICUR & & & $£ \mid 786$ \\
\hline ICUR, incremental cost-utility ratio. & &
\end{tabular}

TABLE 5 I Incremental costs and QALYs, AM+CBA compared with AM - NHS perspective, by subgroup

\begin{tabular}{|c|c|c|c|c|c|c|c|}
\hline & \multicolumn{2}{|l|}{$\mathbf{A M}$} & \multicolumn{2}{|c|}{$A M+C B A$} & \multirow{2}{*}{$\begin{array}{l}\text { Incremental } \\
\text { cost }(t)\end{array}$} & \multirow{2}{*}{$\begin{array}{l}\text { Incremental } \\
\text { QALYs }\end{array}$} & \multirow[b]{2}{*}{$\operatorname{ICER}(\ell)$} \\
\hline & Cost (t) & QALY & Cost (E) & QALY & & & \\
\hline Males $(n=214)$ & 186.72 & 0.640 & 397.47 & 0.727 & 210.75 & 0.087 & 2422 \\
\hline Females $(n=3 \mid 4)$ & 251.34 & 0.583 & 413.56 & 0.694 & 162.22 & 0.111 & $|46|$ \\
\hline $\begin{array}{l}\text { Older than } 60 \text { years } \\
(n=220)\end{array}$ & 218.53 & 0.563 & 454.15 & 0.690 & 235.62 & 0.127 & 1855 \\
\hline $\begin{array}{l}\text { Younger than } \\
60 \text { years }(n=308)\end{array}$ & 228.11 & 0.620 & 372.65 & 0.714 & 144.54 & 0.094 & 1538 \\
\hline $\begin{array}{l}\text { Duration of pain } \\
\leq 3 \text { years }(n=154)\end{array}$ & 259.35 & 0.681 & 383.74 & 0.749 & 124.39 & 0.068 & 1829 \\
\hline $\begin{array}{l}\text { Duration of pain } \\
>3 \text { years }(n=374)\end{array}$ & 210.74 & 0.559 & 415.23 & 0.688 & 204.49 & 0.129 & 1585 \\
\hline $\begin{array}{l}\text { RMQ score at } \\
\text { baseline } \geq 4 \\
(n=442)\end{array}$ & 236.76 & 0.583 & 427.20 & 0.708 & 190.44 & 0.125 & 1524 \\
\hline $\begin{array}{l}\text { RMQ score at } \\
\text { baseline }<4(n=86)\end{array}$ & 151.53 & 0.729 & 302.18 & 0.722 & 150.65 & -0.007 & $\begin{array}{l}\text { AM+CBA } \\
\text { dominated }\end{array}$ \\
\hline
\end{tabular}

which the estimation of the ICUR was made. Figure 19 shows that the incremental cost and QALY of CBA are both always positive. Therefore CBA is never dominant (i.e. less costly and more effective).

To visually assess the range of variation for the ICER, we also plotted the cost-effectiveness thresholds at $£ 500$ and $£ 4000$. These are the dotted and dashed lines in Figure 19.

As the ICUR is the ratio of incremental costs and QALYs, the slope of the line joining any of the points with the origin is a graphical representation of the ICER. Therefore all points above the $£ 500$ line and below the $£ 4000$ line are points associated with an ICER of between $£ 500$ and $£ 4000$.

We summarised the variations of the ICUR in a cost-effectiveness acceptability curve (Figure 20).
This is the probability that CBA is cost-effective at the decision maker's cost-effectiveness threshold $\lambda$, the willingness to pay for one QALY, plotted here for the range $£ 0-£ 35,000$. The probability of CBA being cost-effective reaches $90 \%$ at about $£ 3000$ and remains at that level or higher above that threshold. At $£ 30,000 \mathrm{CBA}$ has an almost $100 \%$ probability of being considered cost-effective.

\section{Subgroup analysis}

We estimated the cost-utility of CBA by age and sex to test the robustness of recommendations within the general population with LBP. The descriptive analysis of costs and QALYs (see Costs) showed that costs and QALYs are not likely to vary by sex or age. However, back pain severity (RMQ scores) was shown to be associated with higher costs. The subgroup analysis reported here (Table 51) shows confirmatory evidence that age, sex and duration of back pain do not have a large impact 


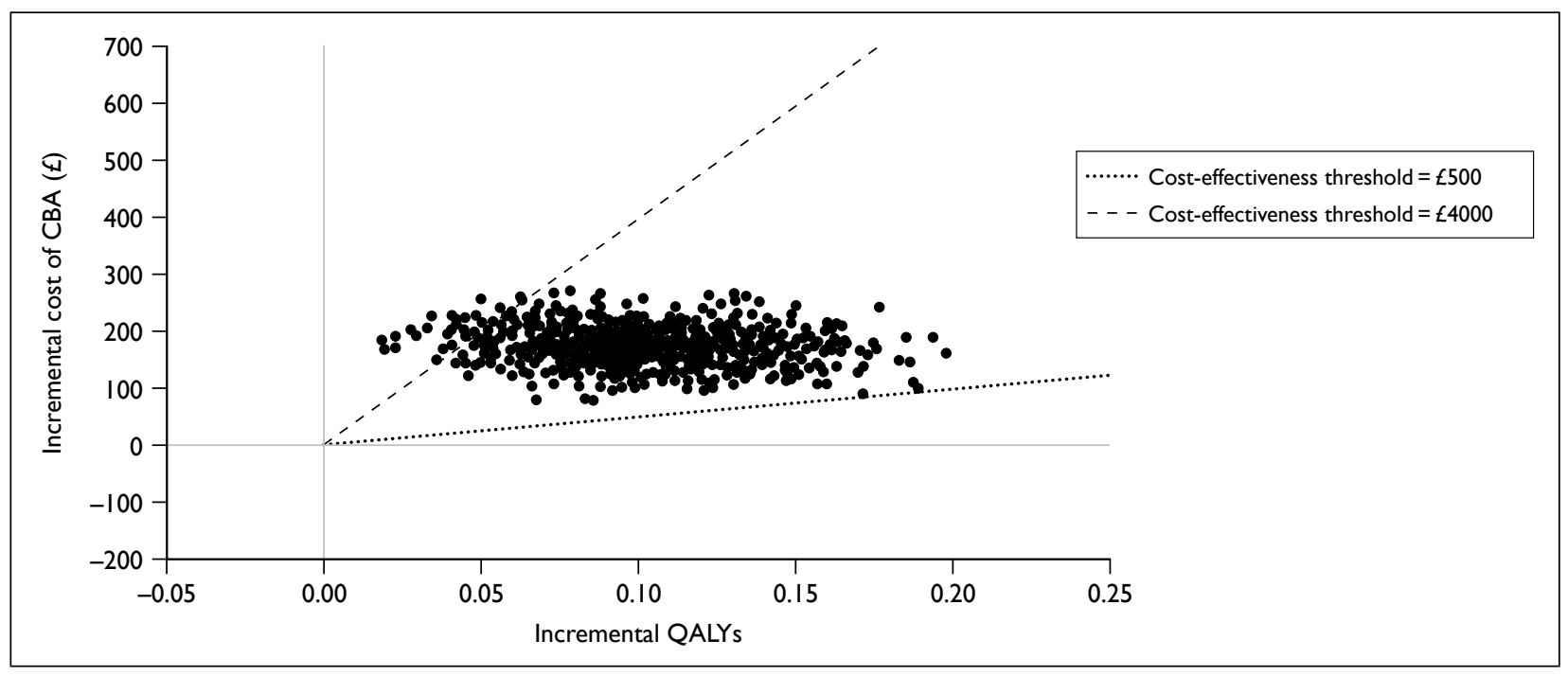

FIGURE 19 Cost-effectiveness plane, incremental costs and incremental QALYs, AM+CBA versus AM - NHS perspective.

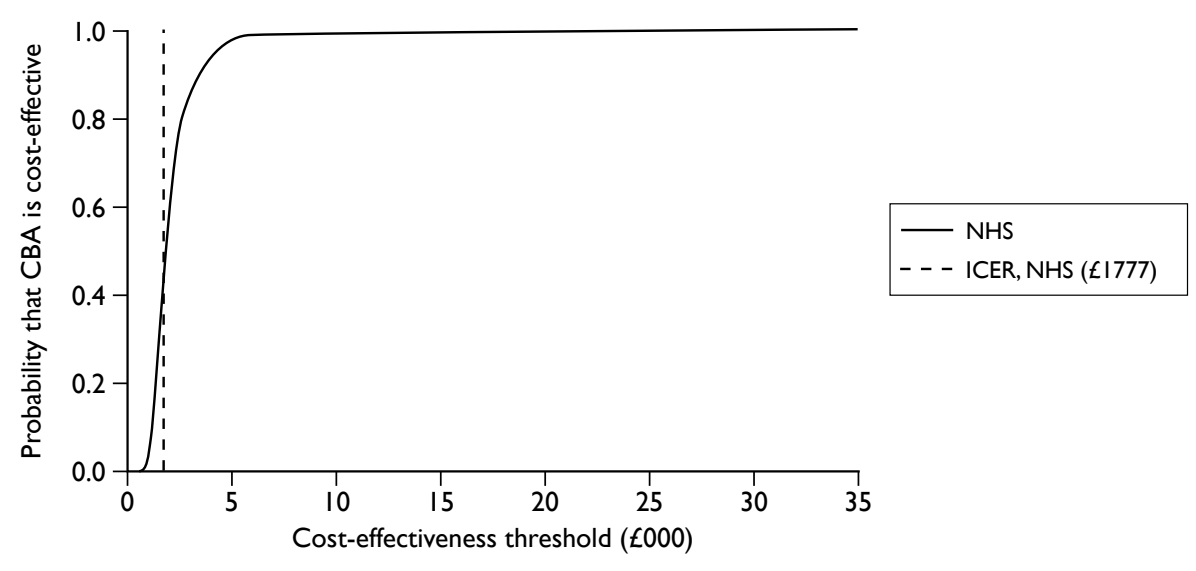

FIGURE 20 Cost-effectiveness acceptability curves, $A M+C B A$ versus $A M-N H S$ perspective.

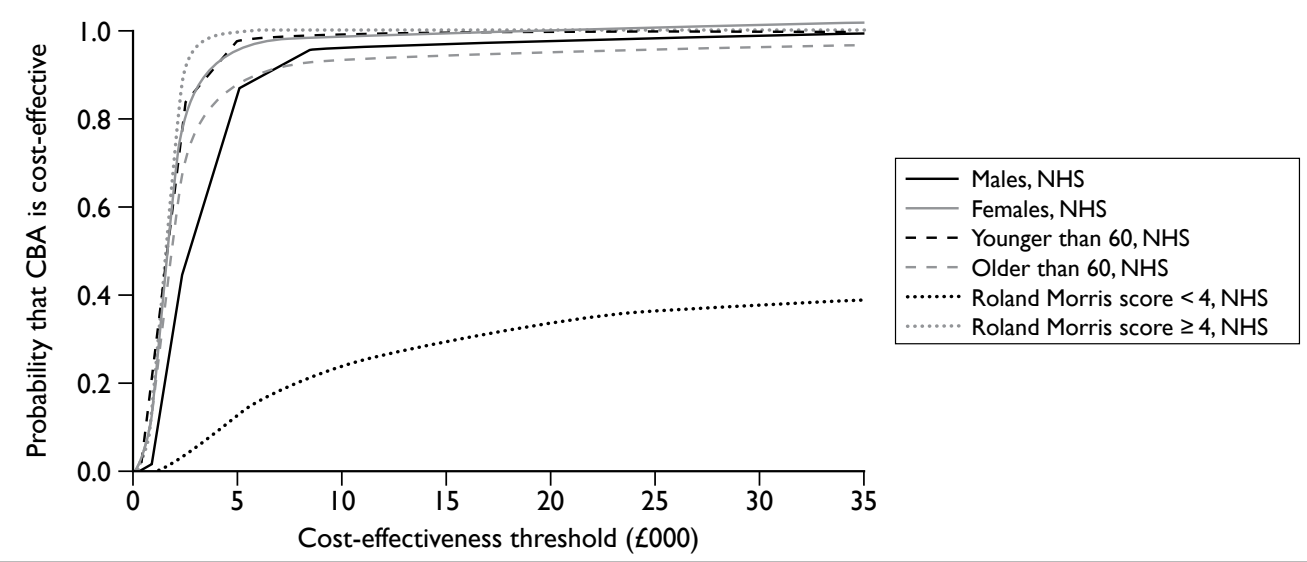

FIGURE 2 I Cost-effectiveness acceptability curves, $A M+C B A$ versus $A M-N H S$ perspective, by subgroup. 
on the cost-effectiveness of CBA, which remains well below currently recognised cost-effectiveness thresholds. However, in the subgroup analysis for RMQ scores we found that CBA was associated with positive incremental costs whilst incremental QALYs were negative in the low-score range (RMQ $<4)$; therefore CBA was 'dominated' by AM in this group, suggesting the CBA may not be costeffective in less severe back pain.

The cost-effectiveness acceptability curves by subgroup (Figure 21) show that the probability of CBA being cost-effective is between $80 \%$ and $99 \%$ for age and sex at cost-effectiveness thresholds of about $£ 5000$. Over this threshold, the probability of CBA being cost-effective remains constant. For individuals with less severe problems from their back pain $(\mathrm{RMQ}$ scores < 4) the probability that CBA is cost-effective is less than $40 \%$ over the customary range of decision-makers' willingness to pay.

\section{General health-care perspective}

From the health-care perspective, CBA was more expensive by approximately $£ 314$, when calculated from within-trial costs. With an improvement in
QALYs of + 0.099, the ICUR was £3093, with CBA still cost-effective at the currently accepted decision-makers' cost-effectiveness threshold (Table 52).

The cost-utility analysis was not sensitive to cost outliers. The ICUR computed using cases with costs lower than the 90th percentile was £2098, with an incremental cost of $£ 189.62$.

\section{Uncertainty}

The incremental costs and QALYs of CBA under the general health-care perspective remain positive, indicating no dominance for CBA (Figure 22).

The probability that the ICUR is below the accepted cost-effectiveness threshold is lower than that under the health-care perspective for low levels of willingness to pay.

At a threshold of $£ 5000$, the probability that CBA is cost-effective is $89 \%$ and this increases to $99 \%$ at about $£ 10,000$, after which it remains constant (Figure 23).

TABLE 52 Incremental costs and $Q A L Y s, A M+C B A$ compared with $A M$ - general health-care perspective

\begin{tabular}{llll} 
& AM & AM+CBA & Difference \\
\hline Total health-care costs & $£ 723.41$ & $£ 1037.78$ & $+£ 314.37$ \\
Total QALYs & 0.604 & 0.703 & +0.099 \\
ICUR & & & $£ 3093$
\end{tabular}

ICUR, incremental cost-utility ratio; QALY, quality-adjusted life-year.

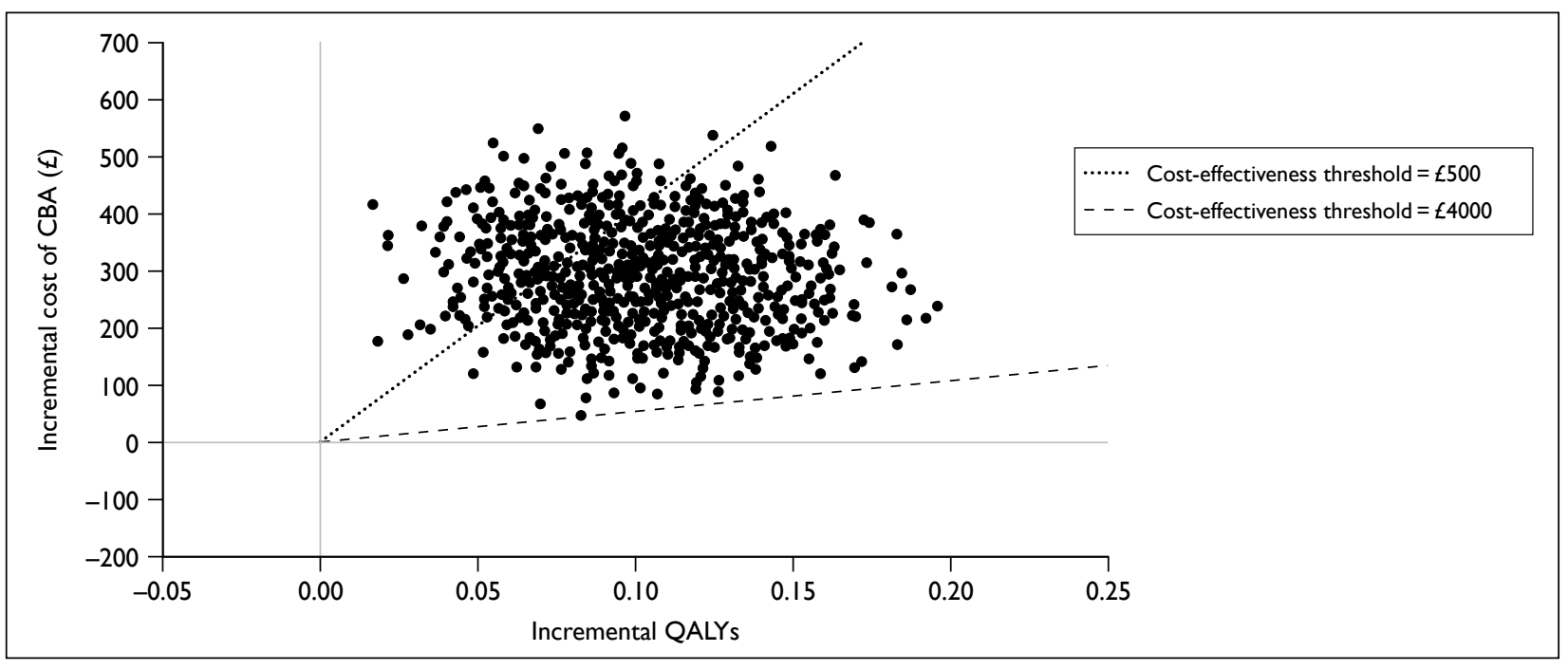

FIGURE 22 Cost-effectiveness plane, $A M+C B A$ versus $A M$ - general health-care perspective. 


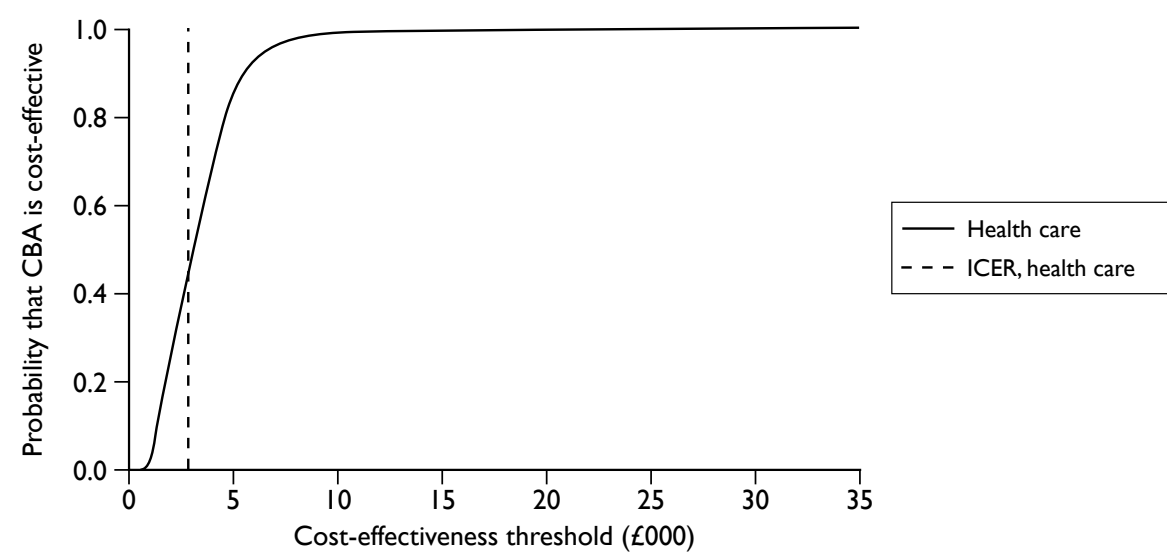

FIGURE 23 Cost-effectiveness acceptability curves, $A M+C B A$ versus $A M$ - general health-care perspective.

TABLE 53 Incremental costs and $Q A L Y s, A M+C B A$ compared with $A M$ - general health-care perspective, by subgroup.

\begin{tabular}{|c|c|c|c|c|c|c|c|}
\hline & \multicolumn{2}{|l|}{ AM } & \multicolumn{2}{|c|}{$A M+C B A$} & \multirow{2}{*}{$\begin{array}{l}\text { Incremental } \\
\text { cost }(t)\end{array}$} & \multirow{2}{*}{$\begin{array}{l}\text { Incremental } \\
\text { QALYs }\end{array}$} & \multirow[b]{2}{*}{$\operatorname{ICER}(\ell)$} \\
\hline & Cost (t) & QALY & Cost $(t)$ & QALY & & & \\
\hline Males $(n=214)$ & 433.89 & 0.640 & 774.22 & 0.727 & 340.33 & 0.087 & 3912 \\
\hline Females $(n=314)$ & 522.26 & 0.583 & 817.20 & 0.694 & 294.94 & 0.111 & 2657 \\
\hline $\begin{array}{l}\text { Older than } 60 \text { years } \\
(n=220)\end{array}$ & 469.61 & 0.563 & 938.46 & 0.690 & 468.85 & 0.127 & 3692 \\
\hline $\begin{array}{l}\text { Younger than } \\
60 \text { years }(n=308)\end{array}$ & 499.96 & 0.620 & 705.33 & $0.7 \mid 4$ & 205.37 & 0.094 & 2185 \\
\hline $\begin{array}{l}\text { Duration of pain } \leq \\
3 \text { years }(n=154)\end{array}$ & 424.64 & 0.681 & 653.55 & 0.749 & 228.91 & 0.068 & 3366 \\
\hline $\begin{array}{l}\text { Duration of pain } \\
>3 \text { years }(n=374)\end{array}$ & 512.74 & 0.559 & 866.76 & 0.688 & 354.02 & 0.129 & 2744 \\
\hline $\begin{array}{l}\text { RMQ score at } \\
\text { baseline } \geq 4 \\
(n=442)\end{array}$ & 499.10 & 0.583 & 861.95 & 0.708 & 362.85 & 0.125 & 2903 \\
\hline $\begin{array}{l}\text { RMQ score at } \\
\text { baseline }<4(n=86)\end{array}$ & 405.15 & 0.729 & 506.48 & 0.722 & 101.34 & -0.007 & $\begin{array}{l}\mathrm{AM}+\mathrm{CBA} \\
\text { dominated }\end{array}$ \\
\hline
\end{tabular}

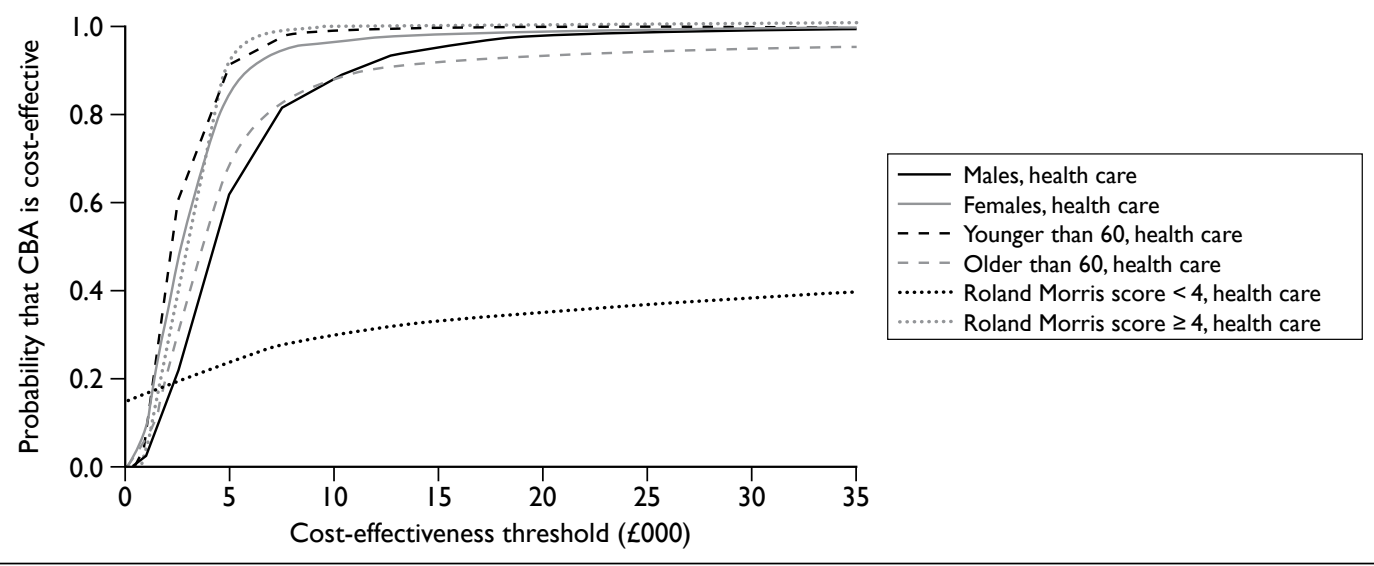

FIGURE 24 Cost-effectiveness acceptability curves, $A M+C B A$ versus $A M$ - general health-care perspective, by subgroup. 


\section{Subgroup analysis}

As for the NHS perspective, the subgroup analysis (Table 53) shows that age, sex and duration of pain do not have a large impact on the cost-effectiveness of CBA, well below currently recognised costeffectiveness thresholds; however for individuals with low back pain severity, CBA is dominated, i.e. more costly and less effective than current practice.
At the $£ 10,000$ cost-effectiveness threshold, CBA is cost-effective with a probability of at least $80 \%$ for all age and sex subgroups, and remains constant above that threshold (Figure 24). However, as for the NHS perspective, the probability that CBA is cost-effective in individuals with RMQ scores $<4$ is low, below $40 \%$ at all accepted willingness-to-pay thresholds. 


\section{Chapter 7 Discussion}

\section{Aims and overview of findings}

The discussion focuses on the interpretation of the findings, internal and external validity of the trial and associated analyses, comparison with other trial data, implications for clinical practice in the NHS and further research.

Low back pain is a common problem. Our aim was to optimise the design of a CBA package that could be delivered within the NHS, and to estimate the effectiveness, cost-effectiveness and acceptability of CBA in addition to best practice advice in primary care using a pragmatic trial design.

This was a definitive large-scale randomised controlled trial, which has demonstrated the longterm effectiveness and cost-effectiveness of CBA in treating subacute and chronic LBP. The clinical and cost-effectiveness outcomes are likely to make this intervention attractive to patients, clinicians and purchasers. Our short-term (3-month) clinical effects are similar to those found in high-quality studies of other therapies including manipulation, exercise and acupuncture. ${ }^{144-146}$ Strikingly, and in contrast to many previous studies, the benefits we observed were maintained over the long term (12 months). The intervention is extremely cost-effective from an NHS and a societal perspective; cost per QALY is less than half that of competing interventions for LBP. Finally, since the intervention can be delivered by existing NHS staff following a brief, 2-day training session, the back skills training programme could be implemented into the NHS with relative ease.

\section{External validity and generalisability of findings}

The external validity of the trial is good. The trial sample was recruited from a range of general practices, including single-handed practices. The regions we recruited ranged from inner city to rural areas, and included areas of significant deprivation. The intervention was delivered by NHS staff and as such, the trial achieved the aim of testing the intervention in an everyday setting and in a sample that was representative of a range of NHS users and providers.

The average age of participants was somewhat older than seen in previous trials. This may be attributable to the ageing of the population. However, in contrast to many previous trials of LBP we did not specify an upper age limit to our entry criteria. Concerns about osteoporosis often predicate the exclusion of older people from trials of spinal manipulation.

The sample is representative of people who will accept the invitation to participate in CBA. This cannot be disentangled from the invitation to participate in the trial. People who were potentially eligible but declined were somewhat younger, but no different in other respects. The most common reason for declining was that people did not want to complete questionnaires during follow-up, and that despite reporting troublesome LBP, people did not consider it bad enough to engage in treatment. Neither of these factors has a significant impact on the interpretation of the findings. There is no suggestion of bias arising from selective loss to follow-up or withdrawal.

Overall, the trial sample was broadly representative of the ethnic mix of the UK. ${ }^{147}$ Four per cent of people recruited were of Asian origin, although this ethnic group was predominantly recruited from the Birmingham cluster.

\section{Critique of methods and threats to internal validity}

We raised concerns with regard to the psychometric properties of the RMQ before starting the trial, and chose to adopt two primary outcome measures at the outset (the RMQ and the MVK scores). Difficulties relating to the ceiling effect of the RMQ have been observed in several studies, and more recent trials of back pain have chosen to define eligibility criteria of having an RMQ score of $>4$ to avert some of these problems. ${ }^{94,148,149}$ However, this approach reduces external validity the RMQ is not used in clinical practice to triage or characterise LBP. Any estimates of the cost- 
effectiveness using a higher RMQ score as an entry criterion should also incorporate an estimate of the costs of screening all people on the RMQ and the quality of life consequences of misclassification with the RMQ. We are not aware of any studies that do. We have presented an analysis of the subgrouping by RMQ score to enable closer comparison with other studies that have used the threshold approach. Interestingly, we report that the CBA intervention is unlikely to be cost-effective in people with low RMQ scores at baseline. This analysis should be treated as exploratory - the difference is almost entirely on differences in cost-expenditure and not associated with clinical outcomes.

Instead of using an RMQ cut-off point, we chose to include people who reported their back pain as at least moderately troublesome. The simplicity and clinical relevance of the troublesome question is widely accepted..$^{150,151}$ The BeST trial included people who have troublesome back pain but who scored $<4$ points on the RMQ. Hence, we had greater potential to encounter analytical problems relating to ceiling effects in the RMQ. We provided strong justification for the betweengroup difference that we wished to detect at the trial protocol stage because we anticipated that the difference between groups would be smaller than in previous trials because of the score range within which we were operating. This point has been supported by Lauridsen et al., ${ }^{125}$ who observed that for individuals who scored $<6$ on the RMQ, an MCID was estimated to be 2, but for those individuals with scores between 6 and 12, the MCID was in the order of 6 . There have been a number of data-driven attempts to determine the MCID for the RMQ, although these should be interpreted with caution because many are based on samples that have used a threshold of $>4 \mathrm{RMQ}$ points to determine eligibility.

The scaling properties of the RMQ also make calculation of numbers needed to treat difficult. Recommendations suggest that a threshold of $30 \%$ change should be used to determine a minimally important change on the measure, ${ }^{124}$ but because the RMQ is a categorical scale, substantial errors are incurred in the calculation (because of rounding errors). We also calculated the numbers needed to treat for the MVK. There is no consensus on the MCID for the MVK, and we used guidance in the chronic pain literature that a $30 \%$ change in a 10-point numeric rating scale is clinically significant. ${ }^{152-154}$ All estimates of numbers needed to treat have to be interpreted with caution.
We specified in the original protocol that we would undertake an interim analysis to compare the psychometric properties of the two primary outcome measures. Standard psychometric approaches did not, however, yield any useful data on which to base a choice - the main difficulty with the RMQ is the skewness in the distribution of raw scores, which severely limits what type of statistical methods can be applied to investigate betweengroup differences, particularly where these involve multilevel modelling. We opted to retain two primary outcomes and, reassuringly, the findings are consistent across both.

A potential criticism is that although we randomised individuals to the treatment options, we did not randomly allocate therapists to participants. It was impossible to achieve this logistically. Also, the method of allocation we studied is reflective of the intervention delivery mechanism if generalised into primary care.

\section{How have we determined that this is a clinically important result?}

Interpretation of any trial is complex. The first step is to rule out that the differences we observed between treatment groups were the result of chance. The study was powered to be definitive, and the statistical significance of the comparisons we drew across a range of measures supports rejecting the null hypothesis (there is no difference between the groups) at low levels of uncertainty. Steps were taken to minimise type I (false-positive) error by including prespecified analysis plans, setting the alpha to 0.01 , and prespecification of primary outcome measures.

Determining clinical significance is more challenging. We specified a priori (protocol version 1) that we wished to detect a difference of 1.4 RMQ change score points between two treatment comparators (i.e. for the new treatment to be improved by about half as much again when compared with the control group). For the purposes of the sample size estimate, this approximated to a standardised difference of 0.35 (assuming data were normally distributed and an SD of 4.0) at 12 months. We modified the trial design before starting recruitment to an unbalanced randomisation, and chose to retain the sample size at the original 700 patients. One eventuality of this scenario was that we might have reduced power by a small amount, or that 
we would only be able to detect larger effect sizes. Ultimately, with retention remaining higher than anticipated and little or no clustering in the data, there was minimal loss of power to detect the differences that we specified originally. We were able to demonstrate a statistically significant effect for our primary outcomes within the range we prespecified as the MCID. Participants who were randomised to CBA received on average, twice as much improvement in outcome across a range of measures. As a consequence, we are confident that we have demonstrated a difference that is both clinically important and statistically significant. The effect size is similar in magnitude to those found in the more robust trials used to inform the draft National Institute for Health and Clinical Excellence (NICE) guidance on LBP. Estimates of the standardised difference for the RMQ have to be interpreted with caution because the distribution of scores was significantly skewed. When we looked at scores integrated over the entire 12 months, the treatment differences were a little smaller. This was because differences in the early phase of the followup were smaller than those being observed at the later stages.

\section{Comments on best practice advice}

Recommendations to support best practice advice are based largely on expert opinion supported by epidemiological data highlighting the importance of physical activity and a limited amount of trial evidence. ${ }^{36,155}$ A trial comparing sham and biomedical advice demonstrated a small short-lived effect of advice on subacute LBP. ${ }^{38}$

The quantitative and qualitative data collected in the best practice advice arm provide insight into the natural history of LBP when managed with a low-intensity intervention. The intervention we selected for the control arm was consistent with international LBP guidance for primary care, and allows comparison across a range of studies that have used a similar control. Back pain improved in the best practice arm. Just over one-quarter of the people who received best practice improved in their symptoms at 1 year (28\%). This is broadly consistent with the natural history of back pain in people - approximately $27 \%$ will be pain-free or improved at 12 months. ${ }^{156-158}$

Comparisons between epidemiological data and the BeST trial data are difficult to draw - we targeted people who had subacute and chronic
LBP and experienced trouble with their backs and in whom the recovery rate might be expected to be lower than in the general population with back pain. Within our trial, The Back Book and brief session of advice had no lasting effect on beliefs about back pain or confidence to deal with the pain. This is disappointing; The Back Book was designed to target these beliefs and clearly fails to achieve any lasting change in mindset for the great majority of people. Reinforcing advice at regular intervals might improve the efficacy of the best practice, but evidence is needed to demonstrate this.

\section{Comments on the cognitive behavioural approach tested in BeST}

Cognitive behavioural interventions take a variety of guises, varying in formulation, intensity, depth and integration with other treatments. The CB method is based on accessing and modifying beliefs that are conceptualised at three levels negative automatic thoughts, 'assumptions' or 'rules', and core beliefs. The latter are deeply held core beliefs. We included the negative automatic thoughts, but not the other belief levels, because their relationship to LBP is not known; and the management of complex beliefs requires specialist psychological expertise and was beyond the scope of group setting we proposed. Guided discovery is the key skill required to elicit beliefs, and is often accompanied by education in skills such as pacing and goal setting.

Just over half of people who were randomised to the CBA intervention reported improvements this is substantially greater than would be expected from natural recovery or from the provision of AM alone. $\mathrm{CBA}$ is used to treat a range of conditions, and we tailored the approach to LBP by using $\mathrm{CB}$ principles to target health beliefs and behaviours associated with poor outcome in this condition. The targets were avoiding movement and activity through fear of pain and further damage, promoting steady build up in physical, leisure and occupational activity. The tailoring involved fusing knowledge on risk factors and effective treatments. It is generally accepted that increasing physical activity is an essential part of the pathway to recovery in LBP, and therefore, we ensured that therapists were trained in up to date knowledge about activity and back pain, as well as CBA. This was particularly important for therapists who were recruited from a psychological discipline. Given 
the importance of physical activity, we stipulated that one of the goals set at the initial assessment should be related to physical activity or exercise. This was well received by participants and in the pilot evaluations we responded to participant comments that they would like more demonstration and checking of exercise and activity. That said, only very limited amounts of time were spent on direct supervision of exercise, and this was very much up to individual choice. We estimate that on average direct supervision of exercise occurred in less than $20 \%$ of participants, for less than $5 \%$ of the intervention time.

\section{Other literature: comparisons of cognitive behavioural therapy with advice interventions in primary care}

Cognitive behavioural approaches have been applied in a variety of ways in back pain - in isolation, alongside as opposed to integrated with other interventions, integrated with other treatments (notably exercise), and in formats in which the educational skills are used but scant attention is paid to eliciting beliefs. The recent draft NICE guidance on LBP has attempted to classify interventions, distinguishing between psychological interventions and combined psychological/physical interventions. ${ }^{159,160}$ As our primary intention was to challenge beliefs that would effect a change in a range of behaviours, and over $95 \%$ of the intervention contact time was directed toward psychological goals, we would label our intervention as psychological.

During the lifespan of the BeST study, several variants of $\mathrm{CB}$ approaches have been tested in comparison with best practice advice.

Two additional trials provide insight into the effectiveness of best practice advice as a comparator.

Johnson et al. ${ }^{149}$ selected people if they had an $\mathrm{RMQ}>5$ and back pain of longer than 3 months' duration, but excluded those who had consulted for symptom management in the last 6 months. The interventions tested were a booklet and audio advice about back pain (based on $\mathrm{CB}$ principles) in comparison with the advice plus group CBA sessions. The estimates of betweengroup differences were smaller than we observed in BeST although they bordered on statistical and clinical significance at some time points. The sample size was small, making interpretation very difficult. Many trials of LBP have chosen unrealistically large effect sizes, and have resulted in trials underpowered to detect smaller differences that are substantially important when the publichealth impact of LBP is considered. In comparison with BeST, there are some differences in the intervention - the training and intervention was provided by physiotherapists, and the degree of psychological input and compliance with British Association for Counselling and Psychotherapy definitions of a $\mathrm{CB}$ intervention is unclear. The control advice intervention may have been of a greater intensity, sufficient to match the group treatments. The participants of the Johnson trial reported more chronic symptoms, although our subgroup data indicate that this should not be an important variant in the treatment response.

Jellema et al. ${ }^{161}$ trained GPs to identify and intervene on psychosocial factors. The intervention was a 20-minute $\mathrm{CB}$ session, in addition to the original consultation. GPs were trained to use standardised questions to elicit information on fear avoidance, beliefs about the causes of LBP, worries, catastrophising and other behaviours. Treatment techniques included information giving, and goal setting, supplemented by The Back Book. A followup appointment was made if persistent LBP was anticipated. GPs were given 5 hours of training in the method. The control arm received the usual care of 'wait and see', graded uptake of activities and pain medication. There was no difference in the effectiveness of the strategies, and no change in either fear avoidance or coping. The intervention may have been too brief, and process evaluation suggests that the GPs found difficulty in identifying psychosocial risk factors. The intervention was also less intensive, and some may question whether it is a CB intervention at all.

\section{Comparison of cognitive behavioural interventions with other physical interventions in primary care}

The Hands on, Hands off trial of Hay et al. ${ }^{148}$ describes a pain management programme for primary care in Staffordshire, UK, that was delivered on a one-to-one basis that included some of the types of education skills used in BeST. The overall duration of the programme was much 
shorter, suggesting that belief elicitation may have been a smaller component of the programme. The intervention was no more effective than the comparator, which was physiotherapy including manipulation. This makes interpretation of this trial difficult. Both treatments may have been equally effective, or equally ineffective. Frost $e t$ al.'s Oxfordshire Low Back Pain Trial ${ }^{37}$ reported that physiotherapy delivered within the NHS is no more effective than a best practice advisory session. Comparison with BeST results is also complicated because Hay et al. ${ }^{148}$ recruited people with back pain of short duration, excluding those with pain for longer than 12 weeks. The one-to-one CB intervention tested in the Hands on, Hands off trial was more expensive than routine physiotherapy. ${ }^{162}$

Two studies from the Netherlands have tested interventions that are very intensive. Smeets et al. ${ }^{47,162}$ recruiting people with chronic LBP reported that a highly intensive $\mathrm{CB}$ intervention was more effective than a waiting list control, but of similar effectiveness to an intensive exercise training protocol. An average of 22 group CB therapy sessions were delivered per participant. Van de Roer et al. ${ }^{163}$ drew a comparison between physiotherapy and an intensive cognitive group programme (up to 30 sessions for the group-based intervention, and an average of nine sessions of physiotherapy). There was no difference in costeffectiveness between the two approaches (clinical outcomes have not been reported to date).

Critchley et al..$^{86}$ investigated the effectiveness of a pain management programme that was predominantly supervised exercise, and additional sessions on goal setting and positive coping strategies. There is no mention of eliciting and challenging beliefs. There were no differences between individual sessions of physiotherapy, pain management groups, or spine stabilisation exercises, with all groups demonstrating improvement. Again it is difficult to draw strong comparisons with the BeST trial or conclusions on effectiveness, as there was no usual care or best practice arm.

\section{Comparison of other physical treatments with advice only in primary care}

Our short-term (3-month) clinical effects are similar to those found in high-quality studies of other therapies including manipulation exercise and acupuncture. ${ }^{86}$ Strikingly, and in contrast to many previous studies, the benefits we observed were maintained over the long term (12 months).

The UK BEAM trial compared exercise and manipulation (alone and in combination) with best practice advice. ${ }^{94}$ They found that a spinal manipulation package produced a small to moderate benefit at 3 months and by a smaller but still statistically significant margin at 1 year, that exercise produced a small benefit at 3 months but not at 1 year, and that manipulation followed by exercise produced a small to moderate benefit at 3 months and a smaller benefit at 1 year.

Unlike acupuncture, CBA had a broad range of effects. In a well-conducted definitive trial, acupuncture reduced pain, but did not impact on disability outcomes. ${ }^{143}$ The CBA tested in BeST impacted on a range of outcomes including disability, pain, health-related quality of life and general health status. We detected a larger effect in global health measures and health-related quality of life than in other trials of physical interventions, suggesting that the CBA has effects outside the spine and so has potential for greater overall benefit than those more localised treatments.

\section{Cost-effectiveness}

Despite a reasonable number of reports on the cost of behavioural interventions in the literature, relatively few studies are of sufficient quality to guide policy decisions. In the absence of wellconducted comprehensive decision analyses, studies of cost-utility, conducted alongside randomised controlled trials provide the best evidence. ${ }^{86,94,164-167}$ When drawing comparisons with trials that have tested interventions against a best practice advice or usual care arm, the BeST intervention is extremely cost-effective from both an NHS and a patient perspective; cost per QALY is less than half that of competing interventions for LBP (Table 54).

The Oxfordshire Low Back Pain trial concluded that best practice advice was more cost-effective than routine physiotherapy. ${ }^{168}$ There is little difference in cost between CB and alternative intensive physical interventions. Critchley et al. ${ }^{86}$ and Johnson et al. ${ }^{149}$ have both demonstrated that cost-effectiveness can be attained using group sessions even when the additional clinical benefits of the intervention are small or negligible. 
TABLE 54 Cost-effectiveness data from trials testing interventions for back pain in primary care

\begin{tabular}{|c|c|c|c|c|}
\hline & Intervention & Comparator & $\begin{array}{l}\text { Perspective, } N \text {, } \\
\text { time frame }\end{array}$ & Cost/cost-effectiveness \\
\hline $\begin{array}{l}\text { Whitehurst } \\
2007^{165}\end{array}$ & $\begin{array}{l}\text { Brief pain management } \\
\text { programme addressing } \\
\text { psychosocial risk factors }\end{array}$ & $\begin{array}{l}\text { Physiotherapy } \\
\text { (mobilisation, } \\
\text { manipulation and soft } \\
\text { tissue treatment) }\end{array}$ & $\begin{array}{l}\text { Generic health } \\
\text { care; } N=299 \\
\text { TF= I year }\end{array}$ & $\begin{array}{l}\text { Brief pain management } \\
\text { (CB) not cost-effective; } \\
\text { ICER }=£ 2800 \text { for } \\
\text { physiotherapy }\end{array}$ \\
\hline $\begin{array}{l}\text { Jellema } \\
2007^{167}\end{array}$ & $\begin{array}{l}\text { Minimal intervention aimed } \\
\text { at psychosocial factors }\end{array}$ & Usual care & $\begin{array}{l}\text { NHS; } M I S=116 \\
\text { UC= } 134 ; \\
T F=1 \text { year }\end{array}$ & Inconclusive \\
\hline $\begin{array}{l}\text { van der Roer } \\
2008^{165}\end{array}$ & $\begin{array}{l}\text { Intensive group training - } \\
\text { physiotherapy + behavioural } \\
\text { component }\end{array}$ & $\begin{array}{l}\text { Guideline individual } \\
\text { physiotherapy } \\
\text { sessions }\end{array}$ & $\begin{array}{l}\text { Societal; } N=102 \\
T F=1 \text { year }\end{array}$ & $\begin{array}{l}\text { ICER }=€ 500 \text { to } € 5150 \\
\text { depending on assumptions }\end{array}$ \\
\hline $\begin{array}{l}\text { Rivero-Arias } \\
2006^{166}\end{array}$ & Active management advice & Physiotherapy & $\begin{array}{l}\text { NHS; } N=286 \\
T F=I \text { year }\end{array}$ & $I C E R=£ 3010$ \\
\hline \multirow[t]{2}{*}{ BEAM $2004^{94}$} & $\begin{array}{l}\text { Exercise } \\
\text { Spinal manipulation }\end{array}$ & \multirow[t]{2}{*}{$\begin{array}{l}\text { Active management } \\
\text { and The Back Book }\end{array}$} & \multirow[t]{2}{*}{$\begin{array}{l}\text { Health care; } \\
N=1287 \\
T F=1 \text { year }\end{array}$} & $\begin{array}{l}\text { AM+ exercise dominated; } \\
\mathrm{AM}+\text { manipulation }+ \\
\text { exercise } £ 3800\end{array}$ \\
\hline & Manipulation and exercise & & & $\mathrm{BC}+$ manipulation $£ 8700$ \\
\hline $\begin{array}{l}\text { Ratcliffe } \\
2006^{168}\end{array}$ & Acupuncture & Usual care & $\begin{array}{l}\text { Societal; } N=24 I \\
T F=2 \text { years }\end{array}$ & ICER $=£ 424 \mid$ \\
\hline
\end{tabular}

\section{Addressing concerns about training and delivery}

We trained NHS therapists and nurses from a variety of professional backgrounds, although NHS physiotherapists were the predominant professional group delivering the intervention. Despite concerns from the practice community that the training course was short ( 2 days), and that nonpsychological disciplines should not or cannot be taught CB skills, the evidence generated by the trial demonstrates improvements in the psychological traits we were targeting. The professional training for many allied health and nursing professions includes motivation, self-management, health education and provision of group treatments. There was no indication that the professional background of therapists was a determinant of therapist effects. We provided mentoring support to all professionals, although many therapists were largely self-sufficient in the treatment delivery. Future implementation will need to address not only training but provision of mentoring, which could be achieved by specialist therapists or in collaboration with local psychological services. The pattern of outcome across the intermediary outcomes and the disease-specific outcomes is consistent with, although not confirmatory of, the treatment mechanism being mediated by change in beliefs about the benefits of physical activity, and in confidence to participate in activities despite pain. An additional concern is whether the healthservice contacts that were generated by the research project (the two research nurse appointments) would influence the effectiveness of the CBA. As both arms received the two assessments and the advice session (which was included in the second assessment) there is no basis to believe that the findings are not attributable to CBA.

\section{Other things we have learnt about cognitive behavioural approach interventions}

We gained some insight into how CBA might work in LBP. Changes in intermediary outcomes were consistent with a hypothesis that CBA reduces fear avoidance and improves pain self-efficacy. However, within the quantitative data set, no solid inference can be made about a mechanism of effect as there is no information on the temporal sequence of changes. The qualitative data set provides further data to support the hypothesis that recognition of negative thoughts and behaviours is a key to enabling recovery, and the importance of re-establishing routine physical, leisure and occupational activity. 
The relationship with the therapist was raised by a number of individuals who were interviewed. Most comments were in a positive light, although on occasion, this was not the case. However, within the quantitative analysis, we found very little evidence that variation in treatment outcome was influenced by individual therapists. There are a number of potential explanations. Although there was quite a large number of therapists involved in the delivery of the intervention, in practice a core of therapists delivered to a large number of groups. As a consequence the variance structure was complex and modelling required extending current methods and in some instances failed to achieve a good fit. Group and therapist effects were to a degree confounded. We had structured the intervention purposefully so that the same therapist would be responsible for delivering all assessments and sessions within each cycle of CBA. Additionally, each group was discrete. Only on rare occasions did a participant swap between group/therapist. As a consequence, the effects of participant, group and therapist are challenging to differentiate.

We measured the competence of most therapists in delivering the intervention by audio-taping sessions and assessing competence against prespecified and internationally agreed criteria. Although competence varied between therapists, therapist effects were still not influential. Possible explanations are the structured approach to the intervention in both the participant training manual and the format of the group sessions, which means that participants may be exposed to cognitive training aspects regardless of the ability of the therapists to use skills such as Socratic questioning.

Previous qualitative research has highlighted the potential importance of 'group' effects in determining outcome, and the therapeutic effect of both talking with people with a similar condition and drawing comparison with others with a similar diagnostic label. ${ }^{169}$ Similar themes emerged from the qualitative analysis, but the quantitative analysis mitigates against group effects as being a major factor in explaining how the intervention works. We made no attempt to group participants of similar backgrounds together (for example men and women), meaning that the variability in participant profile was high in all groups.

We prespecified a per protocol analysis to explore dose dependency. We hypothesised that individuals would need to attend the assessment and three sessions to ensure the main messages of the intervention were embedded. Compliance with the intervention at this prespecified level was reasonable (65\%), but effectiveness was not influenced by compliance in the way that we had measured it.

We ran a number of subgroup analyses to determine whether pain severity, duration or fear avoidance at baseline were predictors of treatment outcomes at 12 months. These analyses were all prespecified and based on stringent interpretation of interaction tests to minimise false-positive findings. Nevertheless, the findings should be treated as exploratory. The factors which emerged were fear avoidance at baseline and then impact was seen only in MVK (disability) scores - those people who showed least fear avoidance at baseline had larger responses to the intervention. Also, pain severity at baseline had a weak association with treatment outcomes measured by the RMQ at 12 months - the treatment effect measured by the RMQ may be larger in those with moderate pain at baseline. Both observations may be useful for generating hypotheses for future research, but should be used with caution in informing health technology appraisal.

Within one cluster there were several general practices that had a high proportion of people from minority ethnic groups. These practices were also based in areas of high socioeconomic deprivation. Participants who were referred into groups from these practices had a lower rate of attendance on the programme $(50 \%$ compliance compared with $67 \%$ in the trial overall). The therapists involved in facilitating these programmes reported that although there was enthusiasm to attend, participants were often unable to attend because of difficult work and social situations, such as child-care cover. Even though these practices had high numbers of people from ethnic minorities, it became apparent that within the trial it was not practical to be able to fill a group with participants who spoke the same language and to be able to find and train enough therapists to cover languages that might be used. We considered using a translator but felt that the discursive nature of the group intervention would have been compromised as the therapist would not have been easily able to facilitate discussion between group participants. As a result of the low number of health professionals from ethnic minority backgrounds, these issues are likely to be present even outside a trial environment and a flexible approach will be needed to deliver a CBA in some populations. It may be that alternative 
programmes requiring fewer visits overall may overcome difficulties in attendance. In addition, to meet cultural or language needs, the intervention may need to be run on a one-to-one basis or via different mediums such as more comprehensive written materials, video format or the use of internet interventions that seek to teach the same self-management skills.

\section{Future research questions}

Future research on implementation of the CBA programme will help to ensure that the benefits we found can be translated into a reduction in back pain disability. Further work is needed to assess the added value of adding the group activity to the assessment session alone. This may be more acceptable and may have similar effectiveness and be more cost-effective than our current CBA package. Our CBA approach was developed specifically for LBP; however, this is rarely seen in isolation. Further work is needed to test this approach for more widespread musculoskeletal pain. We hypothesise that the BeST approach could be adapted to treat a range of musculoskeletal conditions. Our sample included many people who experience pain in other areas of the body, not just the back. The substantial impact on health-related quality of life might be explained by participants using the skills learnt to manage pain for other regions of the body. 


\section{Chapter 8 Conclusion}

$\mathrm{T}$

he BeST trial confirms the findings

of efficacy trials testing very similar interventions: ${ }^{19,42,45,170-173}$ that a tailored group CBA is effective in the management of LBP in primary care. The results extend the knowledge base to demonstrate sustained clinical effects over a 1-year period in a broad range of outcomes, and that a range of health professionals with appropriate training can deliver the intervention. Our shortterm (3-month) clinical effects are similar to those found in high-quality studies of other therapies, including manipulation and exercise ${ }^{94}$ Strikingly, and in contrast with many previous studies, the benefits we observed were maintained over the long term (12 months). The intervention is extremely cost-effective from an NHS and a societal perspective; cost per QALY is less than half that of competing interventions for LBP. Finally, since the intervention can be delivered by existing NHS staff following a brief, 2-day training session, the back skills training programme could be implemented into the NHS with relative ease. Our analyses were completed after the draft NICE guidelines on LBP were published. We anticipate that any future revision of the guidelines would include this intervention. There now appears to be a strong case to roll the training programme out to NHS practitioners for implementation within the current structures and pathways used to manage back pain. 



\title{
Acknowledgements
}

$\mathrm{W}$

e would like to acknowledge and thank all the therapists involved in delivering the trial intervention.

The following experts provided independent review of the intervention format during development of the protocol; Johan W. S. Vlaeyen, Tamar Pincus, Julie H. Barlow and Elaine Buchanan.

\section{Trial team}

\section{Trial management group}

Professor Sallie Principal Investigator

Lamb

Professor Martin Collaborator

Underwood

Miss Zara Clinical Research Fellow

Hansen

Dr Liset Pengel Clinical Trial Manager [General Practice Research Framework (GPRF)]

(left December 2004)

Gill Foster Clinical Trial Manager (GPRF) (left August 2005)

Mrs Emma Clinical Trial Manager

Withers

Dr Margaret

Vickers

Collaborator

(left December 2004)

Mrs Louise

Research Nurse (GPRF)

Letley

Miss Anita

Chauhan

Miss Susie

Carpenter

Study Administrator (GPRF)

(left May 2005)

Study Administrator (GPRF)

(left August 2005)

Miss Rachel Research Nurse

Potter

Dr Ranjit Lall Statistician

Dr Anne Daykin Clinical Research Fellow

(left June 2004)

\section{Trial steering committee}

Professor Sarah

Stewart-Brown

Professor Cath

Chair (stepped down - no

Sackley

Mr Roy Jones

Chair

Lay Member

\author{
Dr Chris \\ McCarthy \\ Professor Julie Independent Member \\ Barlow \\ Dr Tamar (Replaced - unable to attend) \\ Pincus \\ Professor Independent Member \\ Stephen Joseph
}

\section{Data monitoring committee}

Professor Robert Hills Chair

Professor Gary Macfarlane

Professor Paul Watson

\section{Data management group}

Mrs Emma Withers

Miss Lisa Craven

Miss Sophie Page

\section{Statistician}

Dr Ranjit Lall

\section{Health economists}

Emanuela Castelnuovo

Professor Ala Szczepura

Mr Mike Clark

\section{Research team}

Judy Briant

Valerie Brueton

John Coult

Sue Counsell

Alison Dearson

Julia Edwards

Anne Hall

Lesley Hand

Zara Hansen

Katie Holt

Dr Kate Horsler

Christine Jarvey

Sandra Jepson

Andy Law
Research Nurse

Senior Nurse

Research Physiotherapist Research Nurse

Research Physiotherapist

Research Physiotherapist

Regional Trainer

Regional Trainer

Research Physiotherapist Research Physiotherapist

Research Clinical Psychologist Physiotherapist

Research Physiotherapist

Research Physiotherapist 


$\begin{array}{ll}\text { Louise Letley } & \text { Trial Senior Nurse } \\ \text { Janet Lowe } & \text { Research Physiotherapist } \\ \text { Pat Marsh } & \text { Research Nurse } \\ \text { Dymphna } & \text { Research Nurse } \\ \text { Medlock } & \\ \text { Jo-Anne Miles } & \text { Research Nurse } \\ \text { Olivia Neeley } & \text { Research Nurse } \\ \text { Vivien Nichols } & \text { Research Physiotherapist } \\ \text { Tracey O’Brien } & \text { Research Physiotherapist } \\ \text { Mary Ogden } & \text { Research Physiotherapist } \\ \text { Grace Pearn } & \text { Research Physiotherapist } \\ \text { Rachel Potter } & \text { Research Nurse } \\ \text { Emma Rayfield } & \text { Research Nurse } \\ \text { Simon Rowland } & \text { Research Physiotherapist } \\ \text { Judy Rushmer } & \text { Research Nurse } \\ \text { Nicola Sloan } & \text { Research Physiotherapist } \\ \text { Barbara Stewart } & \text { Research Nurse } \\ \text { Dr Sam } & \text { Research Health Psychologist } \\ \text { Thompson } & \\ \text { Rachel Turnbull } & \text { Occupational Therapist } \\ \text { Gill Walker } & \text { Occupational Therapist } \\ \text { Sue Webb } & \text { Research Nurse } \\ \text { Anna Williams } & \text { Research Nurse and Regional } \\ & \text { Trainer } \\ \text { Mark Woolvine } & \text { Research Physiotherapist } \\ & \end{array}$

\section{Contribution of authors}

Sallie E. Lamb (chief investigator) was grant holder and main author of the original protocol. She contributed to the design of the intervention and oversaw project conduct, analysis and reporting. She drafted sections of the report and academically edited the overall final report. Ranjit Lall (study statistician) was responsible for the statistical analysis and reporting. She drafted some sections and contributed to and approved the final manuscript. Zara Hansen designed the intervention and was responsible for its quality control, analysis and reporting. She drafted some sections and contributed to and approved the final report. Emanuela Castelnuovo (health economist) was responsible for the cost-effectiveness analysis and reporting. She drafted some sections and contributed to and approved the final manuscript. Emma J. Withers (study manager) was responsible for the set up and for managing data. She contributed to and approved the final analysis and formatted the final report. Vivien Nichols (physiotherapist) contributed to recruitment, data analysis and reporting. She drafted the qualitative sections and contributed to and approved the final report. Frances Griffiths designed and supervised the qualitative study, drafted the qualitative sections and contributed to and approved the final report. Rachel Potter (head research nurse) was responsible for managing recruitment; she drafted some sections and contributed to and approved the final report. Ala Szczepura (grant holder) contributed expertise in health economics and health services research and contributed to and approved the final report. Martin Underwood (grant holder) contributed expertise in primary care, low back pain and trials. He assisted with data interpretation and contributed to and approved the final report. 


\section{References}

1. Maniadakis N, Gray A. The economic burden of back pain in the UK. Pain 2000;84(1):95-103.

2. Mason V. The prevalence of back pain in Great Britain. London: HMSO Office of Population Censuses and Surveys; 1994.

3. Walsh DA, Radcliffe JC. Pain beliefs and perceived physical disability of patients with chronic low back pain. Pain 2002;97(1-2):23-31.

4. Dionne C. CE Low back pain. In Combie IK. Croft P, Linton S, LeResche L, von Korff M, editors. Epidemiology of pain. Seattle: International Association for the Study of Pain; 1999.

5. Pengel LH, Herbert RD, Maher CG, Refshauge KM. Acute low back pain: systematic review of its prognosis. BMJ 2003;327(7410):323

6. Schneider S, Schmitt H, Zoller S, Schiltenwolf M. Workplace stress, lifestyle and social factors as correlates of back pain: a representative study of the German working population. Int Arch Occup Environ Health 2005;78(4):253-69.

7. Macfarlane G, Jones G, Hannaford P. Managing low back pain presenting to primary care: where do we go from here? Pain 2006;122(3):219-22.

8. McCormick A, Fleming D, Charlton J. Morbidity statistics from general practice: fourth national study 1991-1992; series MB5 (No. 3). London: HMSO Office of Population Censuses and Surveys; 1995.

9. Arthritis Research Campaign. Arthritis: the big picture. London: ARC; 2002.

10. Waddell G, Feder G, McIntosh A, Lewis M, Hutchinson A. Low back pain: clinical guidelines and evidence review. London: Royal College of General Practitioners; 1996.

11. Croft PR, Macfarlane GJ, Papageorgiou AC, Thomas E, Silman AJ. Outcome of low back pain in general practice: a prospective study. $B M J$ 1998;316(7141):1356-9.

12. Maetzel A, Li L. The economic burden of low back pain: a review of studies published between 1996 and 2001. Best Pract Res Clin Rheumatol 2002;16(1):23-30.
13. Merskey H. Pain terms: a list with definitions and notes on usage recommended by the International Association for the Study of Pain sub-commitee on taxonomy. Pain 1979;6:249-52.

14. Grotle M, Brox J, Glomsrød B, Lønn J, Vøllestad N. Prognostic factors in first-time care seekers due to acute low back pain. Eur J Pain 2007;11(3):290-8.

15. Dunn KM, Jordan K, Croft PR. Characterizing the course of low back pain: a latent class analysis. Am J Epidemiol 2006;163(8):754-61.

16. Deyo RA. Low-back pain. Sci Am 1998;279(2):48-53.

17. Thomas E, Silman AJ, Croft PR, Papageorgiou AC, Jayson MI, Macfarlane GJ. Predicting who develops chronic low back pain in primary care: a prospective study. BMJ 1999;318(7199):1662-7.

18. Carragee EJ, Alamin TF, Miller JL, Carragee JM. Discographic, MRI and psychosocial determinants of low back pain disability and remission: a prospective study in subjects with benign persistent back pain. Spine J 2005;5(1):24-35.

19. Linton SJ, Andersson T. Can chronic disability be prevented? A randomized trial of a cognitivebehavior intervention and two forms of information for patients with spinal pain. Spine 2000;25(21):2825-31; discussion 4.

20. Pincus T, Burton AK, Vogel S, Field AP. A systematic review of psychological factors as predictors of chronicity/disability in prospective cohorts of low back pain. Spine 2002;27(5):E109-20.

21. Swinkels-Meewisse IE, Roelofs J, Schouten EG, Verbeek AL, Oostendorp RA, Vlaeyen JW. Fear of movement/(re)injury predicting chronic disabling low back pain: a prospective inception cohort study. Spine 2006;31(6):658-64.

22. Boersma K, Linton SJ. Psychological processes underlying the development of a chronic pain problem: a prospective study of the relationship between profiles of psychological variables in the fear-avoidance model and disability. Clin J Pain 2006;22(2):160-6.

23. Hurwitz EL, Morgenstern H, Chiao C. Effects of recreational physical activity and back exercises on low back pain and psychological distress: findings 
from the UCLA Low Back Pain Study. Am J Publ Health 2005;95(10):1817-24.

24. European guidelines for the management of low back pain. Acta Orthop Scand Suppl 2002;73(305):20-5.

25. Hoogendoorn WE, van Poppel MN, Bongers PM, Koes BW, Bouter LM. Systematic review of psychosocial factors at work and private life as risk factors for back pain. Spine 2000;25(16):2114-25.

26. Lund T, Labriola M, Christensen KB, Bultmann U, Villadsen E. Physical work environment risk factors for long term sickness absence: prospective findings among a cohort of 5357 employees in Denmark. BMJ 2006;332(7539):449-52.

27. Neubauer E, Junge A, Pirron P, Seemann H, Schiltenwolf M. HKF-R 10 - screening for predicting chronicity in acute low back pain (LBP): a prospective clinical trial. Eur J Pain 2006;10(6):559-66.

28. Silman AJ, Jayson MI, Papageorgiou AC, Croft PR. Hospital referrals for low back pain: more coherence needed.J R Soc Med 2000;93(3):135-7.

29. Coyle D, Richardson G. The cost of back pain. In CSAG, Epidemiology Review: the epidemiology and cost of low back pain. Report. London: HMSO; 1994.

30. AHCPR. 1994 Acute low back problems in adults. Clinical Practice Guidelines 14. Rockville, MD: US Department of Health and Human Services; 1994.

31. Koes BW, van Tulder MW, Ostelo R, Kim Burton A, Waddell G. Clinical guidelines for the management of low back pain in primary care: an international comparison. Spine 2001;26(22):2504-13; discussion $13-14$.

32. Nordin M, Balague F, Cedraschi C. Nonspecific lower-back pain: surgical versus nonsurgical treatment. Clin Orthopaed Related Res 2006;443:156-67.

33. van Tulder MW, Scholten RJ, Koes BW, Deyo RA. Non-steroidal anti-inflammatory drugs for low back pain. Cochrane Database Syst Rev 2000;(2):CD000396.

34. Roland M, Waddell J. The Back Book. Norwich: The Stationery Office; 1997.

35. Burton AK, Waddell G, Tillotson KM, Summerton N. Information and advice to patients with back pain can have a positive effect. A randomized controlled trial of a novel educational booklet in primary care. Spine 1999;24(23):2484-91.
36. Liddle S, Gracey J, Baxter G. Advice for the management of low back pain: a systematic review of randomised controlled trials. Man Ther 2007;12(4):310-27.

37. Frost H, Lamb SE, Doll HA, Carver PT, StewartBrown S. Randomised controlled trial of physiotherapy compared with advice for low back pain. BMJ 2004;329(7468):708.

38. Pengel L, Refshauge K, Maher C, Nicholas M, Herbert R, McNair P. Physiotherapist-directed exercise, advice, or both for subacute low back pain: a randomized trial. Ann Intern Med 2007;146(11):787-96

39. Hayden JA, van Tulder MW, Malmivaara A, Koes BW. Exercise therapy for treatment of nonspecific low back pain. Cochrane Database Syst Rev 2005;(3):CD000335.

40. Assendelft WJ, Morton SC, Yu EI, Suttorp MJ, Shekelle PG. Spinal manipulative therapy for low back pain. Cochrane Database Syst Rev 2004;(1):CD000447.

41. Grazebrook K, Garland A, Board of B. What are cognitive and/or behavioural psychotherapies? Paper prepared for a UKCP/BACP mapping psychotherapy exercise. British Association for Behavioral and Cognitive Psychologies 2005. URL: www.babcp.com/silo/files/what-is-cbt.pdf. Accessed 21 November 2009.

42. Guzman J, Esmail R, Karjalainen K, Malmivaara A, Irvin E, Bombardier C. Multidisciplinary rehabilitation for chronic low back pain: systematic review. $B M J$ 2001;322(7301):1511-16.

43. Ostelo RW, van Tulder MW, Vlaeyen JW, Linton SJ, Morley SJ, Assendelft WJ. Behavioural treatment for chronic low-back pain. Cochrane Database Syst Rev 2005;(1):CD002014.

44. Storheim K, Brox JI, Holm I, Koller AK, Bo K. Intensive group training versus cognitive intervention in sub-acute low back pain: short-term results of a single-blind randomized controlled trial J Rehab Med 2003;35(3):132-40.

45. Moore JE, Von Korff M, Cherkin D, Saunders K, Lorig K. A randomized trial of a cognitive-behavioral program for enhancing back pain self care in a primary care setting. Pain 2000;88(2): 145-53.

46. Von Korff M, Balderson BH, Saunders K, Miglioretti DL, Lin EH, Berry S, et al. A trial of an activating intervention for chronic back pain in 
primary care and physical therapy settings. Pain 2005;113(3):323-30.

47. Smeets R, Vlaeyen J, Kester A, Knottnerus J. Reduction of pain catastrophizing mediates the outcome of both physical and cognitive-behavioral treatment in chronic low back pain.J Pain 2006;7(4):261-71.

48. Smeets R, Beelen S, Goossens M, Schouten E, Knottnerus J, Vlaeyen J. Treatment expectancy and credibility are associated with the outcome of both physical and cognitive-behavioral treatment in chronic low back pain. Clin J Pain 2008;24(4):305-15.

49. Campbell NC, Murray E, Darbyshire J, Emery J, Farmer A, Griffiths F, et al. Designing and evaluating complex interventions to improve health care. BMJ 2007;334(7591):455-9.

50. Beck AT. Cognitive therapy and the emotional disorders. New York: International Universities Press; 1979.

51. Pincus T, Vlaeyen JW, Kendall NA, Von Korff MR, Kalauokalani DA, Reis S. Cognitive-behavioral therapy and psychosocial factors in low back pain: directions for the future. Spine 2002;27(5):E133-8.

52. Neubauer E, Junge A. HKF0R 10 - screening for predicting chronicity in active low back pain (LBP). Eur J Pain 2006;10(6):559-66.

53. Linton SJ. A review of psychological risk factors in back and neck pain. Spine 2000;25(9):1148-56.

54. Jensen MP, Turner JA, Romano JM, Karoly P. Coping with chronic pain: a critical review of the literature. Pain 1991;47(3):249-83.

55. Jensen MP, Turner JA, Romano JM, Lawler BK. Relationship of pain-specific beliefs to chronic pain adjustment. Pain 1994;57(3):301-9.

56. Williams DA, Keefe FJ. Pain beliefs and the use of cognitive-behavioral coping strategies. Pain 1991;46(2):185-90.

57. Harkapaa K, Jarvikoski A, Hakala L, Jarvilehto S. Outcome of rehabilitation programmes for employees with lowered working capacity. Disability Rehab 1996;18(3):143-8.

58. Jensen MP, Romano JM, Turner JA, Good AB, Wald LH. Patient beliefs predict patient functioning: further support for a cognitive-behavioural model of chronic pain. Pain 1999;81(1-2):95-104.

59. Swinkels-Meewisse IE, Roelofs J, Verbeek AL, Oostendorp RA, Vlaeyen JW. Fear of movement/(re) injury, disability and participation in acute low back pain. Pain 2003;105(1-2):371-9.
60. Vlaeyen JW, Kole-Snijders AM, Boeren RG, van Eek H. Fear of movement/(re)injury in chronic low back pain and its relation to behavioral performance. Pain 1995;62(3):363-72.

61. Vlaeyen JW, Linton SJ. Fear-avoidance and its consequences in chronic musculoskeletal pain: a state of the art. Pain 2000;85(3):317-32.

62. Goubert L, Crombez G, Van Damme S. The role of neuroticism, pain catastrophizing and pain-related fear in vigilance to pain: a structural equations approach. Pain 2004;107(3):234-41.

63. Crombez G, Vlaeyen JWS, Heuts P, Lysens R. Painrelated fear is more disabling than pain itself: evidence on the role of pain-related fear in chronic back pain disability. Pain 1999;80(1-2):329-39.

64. Vlaeyen JWS, de Jong J, Geilen M, Heuts P, van Breukelen G. The treatment of fear of movement/ (re)injury in chronic low back pain: further evidence on the effectiveness of exposure in vivo. Clin J Pain 2002;18(4):251-61.

65. Hasenbring MI, Plaas H, Fischbein B, Willburger R. The relationship between activity and pain in patients 6 months after lumbar disc surgery: do pain-related coping modes act as moderator variables? Eur J Pain 2006;10(8):701-9.

66. Vlaeyen JWS, Morley S. Active despite pain: the putative role of stop-rules and current mood. Pain 2004;110(3):512-16.

67. Pincus T, Vogel S, Burton AK, Santos R, Field AP. Fear avoidance and prognosis in back pain - a systematic review and synthesis of current evidence. Arthr Rheum 2006;54(12):3999-4010.

68. Lawlor DA, Hopker SW. The effectiveness of exercise as an intervention in the management of depression: systematic review and meta-regression analysis of randomised controlled trials. $B M J$ 2001;322(7289):763-7.

69. Buszewicz M, Rait G, Griffin M, Nazareth I, Patel A, Atkinson A, et al. Self management of arthritis in primary care: randomised controlled trial. $B M J$ 2006;333(7574):879-82A.

70. Sieben JM, Portegijs PJM, Vlaeyen JWS, Knottnerus JA. Pain-related fear at the start of a new low back pain episode. Eur J Pain 2005;9(6):635-41.

71. Robinson ME, Riley JL, Myers CD, Sadler IJ, Kvaal SA, Geisser ME, et al. The coping strategies questionnaire: a large sample, item level factor analysis. Clin J Pain 1997;13(1):43-9.

72. Moseley GL, Nicholas MK, Hodges PW. A randomized controlled trial of intensive 
neurophysiology education in chronic low back pain. Clin J Pain 2004;20(5):324-30.

73. Leino PI. Does leisure-time physical activity prevent low back disorders - a prospective study of metal industry employees Spine 1993;18(7):863-71.

74. Thomas JS, France CB, Sha D, Wiele NV, Moenter S, Swank K. The effect of chronic low back pain on trunk muscle activations in target reaching movements with various loads. Spine 2007;32:E801-8.

75. Verbunt JA, Seelen HA, Vlaeyen JW, van de Heijden GJ, Heuts PH, Pons K, et al. Disuse and deconditioning in chronic low back pain: concepts and hypotheses on contributing mechanisms. Eur J Pain 2003;7(1):9-21.

76. Mayer T, Gatchel R. Functional restoration for spinal disorders: the sports medicine approach. Philadelphia: Lea \& Febiger; 1988.

77. Krismer M, van Tulder M. Low back pain (non-specific). Best Pract Res Clin Rheumatol 2007;21(1):77-91.

78. Liddle SD, Baxter GD, Gracey JH. Exercise and chronic low back pain: what works? Pain 2004;107(1-2):176-90.

79. Hayden JA, van Tulder MW, Malmivaara AV, Koes BW. Meta-analysis: exercise therapy for nonspecific low back pain. Ann Intern Med 2005;142(9):765-75.

80. Staal JB, Hlobil H, Twisk JW, Smid T, Koke AJ, van Mechelen W. Graded activity for low back pain in occupational health care: a randomized, controlled trial. Ann Intern Med 2004;140(2):77-84.

81. Rainville J, Hartigan C, Martinez E, Limke J, Jouve C, Finno M. Exercise as a treatment for chronic low back pain. Spine J 2004;4(1):106-15.

82. Staal JB, Rainville J, Fritz J, van Mechelen W, Pransky G. Physical exercise interventions to improve disability and return to work in low back pain: current insights and opportunities for improvement. J Occup Rehab 2005;15(4):491-505.

83. Waddell G. The back pain revolution. Oxford: Churchill Livingstone; 2004.

84. Croft PR, Dunn KM, Raspe H. Course and prognosis of back pain in primary care: the epidemiological perspective. Pain 2006;122(1-2):1-3.
85. Morrison N. Group cognitive therapy: treatment of choice or sub-optimal option? Behav Cognit Psychother 2001;29(3):311-32.

86. Critchley DJ, Ratcliffe J, Noonan S, Jones RH, Hurley MV. Effectiveness and cost-effectiveness of three types of physiotherapy used to reduce chronic low back pain disability - a pragmatic randomized trial with economic evaluation. Spine 2007;32(14):1474-81.

87. Bodenheimer T, Lorig K, Holman H, Grumbach K. Patient self-management of chronic disease in primary care. J Am Med Assoc 2002;288(19):2469-75.

88. Rose MJ, Reilly JP, Pennie B, Bowen-Jones K, Stanley IM, Slade PD. Chronic low back pain rehabilitation programs: a study of the optimum duration of treatment and a comparison of group and individual therapy. Spine 1997;22(19):2246-51.

89. Jellema P, van der Windt DA, van der Horst HE, Twisk JW, Stalman WA, Bouter LM. Should treatment of (sub)acute low back pain be aimed at psychosocial prognostic factors? Cluster randomised clinical trial in general practice. $B M J$ 2005;331(7508):84.

90. Beck JS. Cognitive therapy, basics and beyond. New York: Guilford Press; 1995.

91. Padesky CA. Socratic questioning: changing minds or guided discovery. Keynote address delivered at the European Congress of Behavioural and Cognitive Therapies, London; 1993.

92. Johnson R, Moores L. Pain management: integrating physiotherapy and clinical psychology practice. In Gifford L, editor. Topical issues in pain 5. Falmouth: CNS Press; 2006.

93. Alam R SJ, Winkley K, Lall R, Ismail K. How effective are non-specialist clinicians, compared to psychological specialists, in delivering psychological interventions to improve blood glucose control in type 2 diabetes. Paper presented at 3rd ASM of UK Society for Behavioural Medicine; University of Warwick, UK; 2007.

94. United Kingdom back pain exercise and manipulation (UK BEAM) randomised trial: cost effectiveness of physical treatments for back pain in primary care. BMJ 2004;329(7479):1381.

95. Airaksinen O, Brox J, Cedraschi C, Hildebrandt J, Klaber Moffett J, Kovacs F, et al. Chapter 4. European guidelines for the management of chronic nonspecific low back pain. Eur Spine J 2006;15(Suppl 2):S192-300. 
96. Roland M, Waddell G, Klaber Moffett J, Burton AK, Main CJ, Cantrell T. The Back Book. Anonymous, editor. Norwich: Stationery Office; 2005.

97. Shen LZ. A practical and efficient approach to Database Quality Audit in Clinical Trials. Drug Info J 2006;40(4):385-93.

98. Linton SJ, Hallden K. Can we screen for problematic back pain? A screening questionnaire for predicting outcome in acute and subacute back pain. Clin J Pain 1998;14(3):209-15.

99. Deyo RA, Battie M, Beurskens AJ, Bombardier C, Croft P, Koes B, et al. Outcome measures for low back pain research. A proposal for standardized use. Spine 1998;23(18):2003-13.

100. Bombardier C, Hayden J, Beaton DE. Minimal clinically important difference. Low back pain: outcome measures.J Rheumatol 2001;28(2):431-8.

101. Garratt AM, Klaber Moffett J, Farrin AJ. Responsiveness of generic and specific measures of health outcome in low back pain. Spine 2001;26(1):71-7; discussion 7 .

102. Roland M, Morris R. A study of the natural history of back pain. Part I: development of a reliable and sensitive measure of disability in low-back pain. Spine 1983;8(2):141-4.

103. Von Korff M, Ormel J, Keefe F, Dworkin S. Grading the severity of chronic pain. Pain 1992;50(2):133-49.

104. Ware J Jr, Kosinski M, Keller SD. A 12-item shortform health survey: construction of scales and preliminary tests of reliability and validity. Med Care 1996;34(3):220-33.

105. Waddell G, Newton M, Henderson I, Somerville D, Main CJ. A fear-avoidance beliefs questionnaire (FABQ) and the role of fear-avoidance beliefs in chronic low back pain and disability. Pain 1993;52(2):157-68.

106. Nicholas M. The pain self-efficacy questionnaire: taking pain into account. Eur J Pain 2007;11(2): 153-63.

107. The EuroQol Group. EuroQol - a new facility for the measurement of health-related quality of life. Health Policy 1990;16(3):199-208.

108. Stratford PW, Binkley JM, Riddle DL, Guyatt GH. Sensitivity to change of the Roland-Morris Back Pain Questionnaire: part 1. Physical Ther 1998;78(11):1186-96.
109. Davidson M, Keating JL. A comparison of five low back disability questionnaires: reliability and responsiveness. Physical Ther 2002;82(1):8-24.

110. Garratt AM, Ruta DA, Abdalla MI, Buckingham KR, I.T. The SF 36 health survey questionnaire: an outcome measure suitable for routine use within the NHS? BMJ 1993;206(6890):1440-4.

111. Williams A. A measurement and valuation of health: a chronicle. Centre for Health Economics Discussion Paper. University of York; 1995.

112. van den Brink M, van den Hout W, Stiggelbout A, Putter H, van de Velde C, Kievit J. Self-reports of health-care utilization: diary or questionnaire? Int J Technol Assess Health Care 2005;21(3):298-304.

113. Peto R, Baigent C. Trials: the next 50 years. Large scale randomised evidence of moderate benefits. BMJ 1998;317(7167):1170-1.

114. Cohen J. Statistical power analysis for behavioural sciences. New York, NY: Academic Press. 1997.

115. Klaber Moffett J, Torgerson D, BellSyer S, Jackson D, Llewlyn-Phillips H, Farrin A. Randomised controlled trial of exercise for low back pain: clinical outcomes, costs, and preferences. BMJ 1999;319(7205):279-83.

116. van Tulder MW, Ostelo R, Vlaeyen JW, Linton SJ, Morley SJ, Assendelft WJ. Behavioral treatment for chronic low back pain: a systematic review within the framework of the Cochrane Back Review Group. Spine 2000;25(20):2688-99.

117. Torgerson DJ, Campbell MK. Cost effectiveness calculations and sample size. $B M J$ 2000;321(7262):697.

118. Pocock S. Clinical trials - a practical approach. Chichester: John Wiley; 1987.

119. Lamb SE, Lall R, Hansen Z, Withers EJ, Griffiths FE, Szczepura A, et al. Design considerations in a clinical trial of a cognitive behavioural intervention for the management of low back pain in primary care: Back Skills Training Trial. BMC Musculoskelet Disord 2007;8.

120. Group C. The revised CONSORT statement for reporting of randomised trials: explanation and elaboration. Ann Intern Med 2001;134(8):663-94.

121. Koch G, Tengen C, Jung W, Amara I. Issues for covariance analysis of dichotomous and ordered categorical data from randomised clinical trials and non-parametric strategies for addressing them. Stat Med 1998;17:1863-92. 
122. Lesaffre E, Rizopoulos D, Tsonaka R. The logistic transform for bounded outcome scores. Biostatistics 2007;8(1):72-85.

123. Senn S. Statistical issues in drug development. London: Wiley and Sons; 1997

124. Ostelo RW, Deyo RA, Stratford P, Waddell G, Croft $\mathrm{P}$, Von Korff M, et al. Interpreting change scores for pain and functional status in low back pain: towards international consensus regarding minimal important change. Spine 2008;33(1):90-4.

125. Lauridsen H, Hartvigsen J, Manniche C, Korsholm L, Grunnet-Nilsson N. Responsiveness and minimal clinically important difference for pain and disability instruments in low back pain patients. BMC Musculoskelet Disord 2006;7:82.

126. Brookes ST, Whitley E, Peters TJ, Mulheran PA, Egger M, Davey Smith G. Subgroup analyses in randomised controlled trials: quantifying the risks of false-positives and false-negatives. Health Technol Assess 2001;5(33).

127. Mead N, Bower P. Patient-centredness: a conceptual framework and review of the empirical literature. Soc Sci Med 2000;51(7):1087-110.

128. McIntosh A, Shaw CF. Barriers to patient information provision in primary care: patients' and general practitioners' experiences and expectations of information for low back pain. Health Expect 2003;6(1):19-29.

129. Reid M. An assessment of health needs of chronic low back pain patients from general practice. J Health Psychol 2004;9(3):451-62.

130. Ong B, Hooper H. Comparing clinical and lay accounts of the diagnosis and treatment of back pain. Sociol Health Illn 2006;28(2):203-22.

131. Gold M, Siegel J, Russell L, Weinstein M. Costeffectiveness in health and medicine. New York: Oxford University Press; 1995.

132. Office for National Statistics. Retail Price Index monthly percentage changes, 1988 to 2008. 2008. URL: www.statistics.gov.uk/statbase/TSDSelection 1.asp. Accessed 17 January 2008.

133. Dolan P, Gudex C, Kind P, Williams A. A social tariff for EuroQol: results from a UK general population survey. Discussion paper 138. University of York: Centre for Health Economics; 1995.

134. Curtis L. Unit costs of health and social care. University of Kent: Personal Social Services Research Unit; 2007.

135. The NHS Executive. Costs TNR. London: NHS Executive; 2008.
136. Prescribing Support Unit. Prescribing Analysis and CosT (PACT) data. URL: www.ic.nhs.uk/statisticsand-data-collections/primary-care/prescriptions/ prescription-cost-analysis-2007. Accessed 29 April 2008.

137. Marsh F, Kremer C, Duffy S. Delivering an effective outpatient service in gynaecology. A randomised controlled trial analysing the cost of outpatient versus daycase hysteroscopy. BJOG 2004;111(3):243-8.

138. Chartered Society of Physiotherapists. Change NAf. Pay scales, ERUS FS 01. 2006. URL: www.csp.org.uk/ uploads/documents/csp_erusfs01_payscales1.pdf. Accessed 17 January 2008.

139. Department of Health. Agenda for change, 2005. URL: www.dh.gov.uk/en/Publicationsandstatistics/ Publications/PublicationsPolicyAndGuidance/ DH_4095947. Accessed 17 January 2008.

140. Willan A, Briggs A. Statistical analysis of costeffectiveness data. Chichester: John Wiley \& Sons, Ltd; 2006.

141. Briggs A, Clark T, Wolstenholme J, Clarke P Missing... presumed at random: cost-analysis of incomplete data. Health Econ 2003;12(5):377-92.

142. Raftery J. Review of NICE's recommendations, 1999-2005. BMJ 2006;332(7552):1266-8.

143. Stinnett A, Mullahy J. Net health benefits: a new framework for the analysis of uncertainty in costeffectiveness analysis. Med Decis Making 1998;18(2 Suppl):S68-80.

144. Thomas K, MacPherson H, Ratcliffe J, Thorpe L, Brazier J, Campbell M, et al. Longer term clinical and economic benefits of offering acupuncture care to patients with chronic low back pain. Health Technol Assess 2005;9(32).

145. UK BEAM Trial Team. United Kingdom back pain exercise and manipulation (UK BEAM) randomised trial: effectiveness of physical treatments for back pain in primary care. BMJ 2004;329(7479): 1377 .

146. Underwood MR, Morton V, Farrin A. Do baseline characteristics predict response to treatment for low back pain? Secondary analysis of the UK BEAM dataset [ISRCTN32683578]. Rheumatology (Oxford) 2007;46(8): 1297-302.

147. Office for National Statistics. URL: www.statistics. gov.uk/cci/nugget.asp?id=455. Accessed 17 January 2008.

148. Hay EM, Mullis R, Lewis M, Vohora K, Main CJ, Watson $\mathrm{P}$, et al. Comparison of physical treatments versus a brief pain-management programme 
for back pain in primary care: a randomised clinical trial in physiotherapy practice. Lancet 2005;365(9476):2024-30.

149. Johnson RE, Jones GT, Wiles NJ, Chaddock C, Potter RG, Roberts C, et al. Active exercise, education, and cognitive behavioral therapy for persistent disabling low back pain - a randomized controlled trial. Spine 2007;32(15):1578-85.

150. Dunn KM, Croft PR. Classification of low back pain in primary care: using "bothersomeness" to identify the most severe cases. Spine 2005;30(16):1887-92.

151. Parsons S, Carnes D, Pincus T, Foster N, Breen A, Vogel S, et al. Measuring troublesomeness of chronic pain by location. BMC Musculoskelet Disord 2006;7:34.

152. Dworkin R, Turk D, Wyrwich K, Beaton D, Cleeland C, Farrar J, et al. Interpreting the clinical importance of treatment outcomes in chronic pain clinical trials: IMMPACT recommendations. J Pain 2008;9(2):105-21.

153. Farrar J, Troxel A, Stott C, Duncombe P, Jensen M. Validity, reliability, and clinical importance of change in a $0-10$ numeric rating scale measure of spasticity: a post hoc analysis of a randomized, double-blind, placebo-controlled trial. Clin Ther 2008;30(5):974-85.

154. Farrar J, Portenoy R, Berlin J, Kinman J, Strom B. Defining the clinically important difference in pain outcome measures. Pain 2000;88(3):287-94.

155. Henrotin Y, Cedraschi C, Duplan B, Bazin T, Duquesnoy B. Information and low back pain management: a systematic review. Spine 2006;31(11):E326-34.

156. Dunn KM, Croft PR. Epidemiology and natural history of low back pain. Eura Medicophys 2004;40(1):9-13.

157. Klenerman L, Slade P, Stanley I, Pennie B, Reilly J, Atchison L, et al. The prediction of chronicity in patients with an acute attack of low back pain in a general practice setting. Spine 1995;20(4):478-84.

158. Somerville S, Hay E, Lewis M, Barber J, van der Windt D, Hill J, et al. Content and outcome of usual primary care for back pain: a systematic review. Br J Gen Pract 2008;58(556):790-7, i-vi.

159. Savigny P, Kuntze S, Watson P, Underwood M, Ritchie G, Cotterell M, et al. Low back pain: the acute management of patients with chronic (longer than six weeks) non-specific back pain. London: National Collaborating Centre for Primary Care and Royal College of General Practitioners; 2009.
160. National Institute for Health and Clinical Excellence. The acute management of chronic (longer than 6 weeks) non-specific low back pain. 2009. URL: www.nice.org.uk/nicemedia/pdf/ LowBackPainFullGuidelineConsultation.pdf. Accessed 17 January 2008.

161. Jellema $P$, van der Windt $D$, van der Horst $H$, Blankenstein A, Bouter L, Stalman W. Why is a treatment aimed at psychosocial factors not effective in patients with (sub)acute low back pain? Pain 2005;118(3):350-9.

162. Smeets R, Severens J, Beelen S, Vlaeyen J, Knottnerus J. More is not always better: costeffectiveness analysis of combined, single behavioral and single physical rehabilitation programs for chronic low back pain. Eur J Pain 2008;13(1):71-81.

163. van der Roer N, van Tulder M, Barendse J, Knol D, van Mechelen W, de Vet H. Intensive group training protocol versus guideline physiotherapy for patients with chronic low back pain: a randomised controlled trial. Eur Spine J 2008;17(9):1193-200.

164. van der Roer N, van Tulder M, van Mechelen W, de Vet H. Economic evaluation of an intensive group training protocol compared with usual care physiotherapy in patients with chronic low back pain. Spine 2008;33(4):445-51.

165. Whitehurst D, Lewis M, Yao G, Bryan S, Raftery J, Mullis R, et al. A brief pain management program compared with physical therapy for low back pain: results from an economic analysis alongside a randomized clinical trial. Arthritis Rheum 2007;57(3):466-73.

166. Rivero-Arias O, Gray A, Frost H, Lamb S, StewartBrown S. Cost-utility analysis of physiotherapy treatment compared with physiotherapy advice in low back pain. Spine 2006;31(12):1381-7.

167. Jellema P, van der Roer N, van der Windt D, van Tulder M, van der Horst H, Stalman W, et al. Low back pain in general practice: cost-effectiveness of a minimal psychosocial intervention versus usual care. Eur Spine J 2007;16(11):1812-21.

168. Ratcliffe J, Thomas K, MacPherson H, Brazier J. A randomised controlled trial of acupuncture care for persistent low back pain: cost effectiveness analysis. BMJ 2006;333(7569):626.

169. Barlow J, Barefoot J. Group education for people with arthritis. Patient Educ Couns 1996;27(3):257-67.

170. van Tulder MW, Ostelo RW, Vlaeyen JW, Linton SJ, Morley SJ, Assendelft WJ. Behavioural treatment for chronic low back pain. Cochrane Database Syst Rev 2000;(2):CD002014. 
171. Kole-Snijders AM, Vlaeyen JW, Goossens ME, Rutten-van Molken MP, Heuts PH, van Breukelen G, et al. Chronic low-back pain: what does cognitive coping skills training add to operant behavioral treatment? Results of a randomized clinical trial. J Consult Clin Psychol 1999;67(6):931-44.

172. Linton SJ, Ryberg M. A cognitive-behavioral group intervention as prevention for persistent neck and back pain in a non-patient population: a randomized controlled trial. Pain 2001;90(1-2):83-90.

173. Morley S, Eccleston C, Williams A. Systematic review and meta-analysis of randomized controlled trials of cognitive behaviour therapy and behaviour therapy for chronic pain in adults, excluding headache. Pain 1999;80(1-2):1-13. 


\section{Appendix I \\ Participant initial approach questionnaire}


In confidence

Potential participant study ID number

\author{
Back Skills Training (BeST) Trial
}

\title{
Participant initial approach questionnaire
}

Back Skills Training (BeST) Trial

The University of Warwick Centre for Primary Health Care
THE UNIVERSITY OF WARWICK
MRC General Practice Research

Framework

A randomised study of treatments for back pain in primary care, funded by the NHS R\&D HTA programme (ISRCTN54717854) 


\section{Back Skills Training (BeST) Trial}

Please complete the following information. It will tell us whether you may be able to take part in this study of treatments for back pain.

1. On what date are you completing this questionnaire?

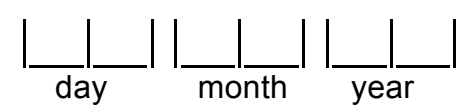

2. What is your date of birth?

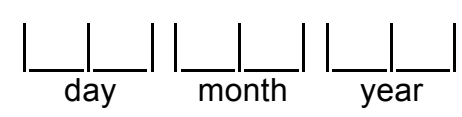

3. What is your sex?

$\begin{array}{ll}\text { Male } & \square_{1} \\ \text { Female } & \square_{2}\end{array}$

4. Have you had any low back pain or symptoms in the past 6 weeks?

$$
\begin{array}{lll}
\text { Yes } & \square_{2} & \text { (Please tick one) } \\
\text { No } & \square_{1} \quad \text { If No, please go to question } 10
\end{array}
$$

5. How often have you had back problems during the past 6 weeks?

$\begin{array}{ll}\text { Everyday } & \square_{1} \text { (Please tick one) } \\ \text { Between everyday and three quarters of the days } & \square_{2} \\ \text { Between three quarters of the days and half of the days } & \square_{3} \\ \text { Between half of the days and a quarter of the days } & \square_{4} \\ \text { Rarely } & \square_{5}\end{array}$

6. How troublesome has your back been during the past 6 weeks?
Not at all troublesome $\quad \square_{1} \quad$ (Please tick one)
Slightly troublesome $\quad \square_{2}$
Moderately troublesome $\square_{3}$
Very troublesome $\quad \square_{4}$
Extremely troublesome $\quad \square_{5}$

7. Are you currently being treated for back pain at a hospital or elsewhere by a consultant, physiotherapist, chiropractor or osteopath?
Yes
$\square_{2} \quad$ (Please tick one)
No 
8. Women only: Are you pregnant?

$\begin{array}{ll}\text { Yes } & \square 2 \\ \text { No } & \square_{1}\end{array}$

The following questions are so that we can ensure we have asked a broad range of people to participate. They do not determine whether you will be eligible to participate.

9. What is your ethnic origin? (Please tick one)

White

${ }_{01} \square$ British

${ }_{02} \square$ Irish

${ }_{03} \square$ Any other White background, please

specify. .....

Mixed

${ }_{04} \square$ White and Black Caribbean

${ }_{05} \square$ White and Black African

${ }_{06} \square$ White and Asian

${ }_{07} \square$ Any other Mixed background, please

specify.

Asian or Asian British

${ }_{08} \square$ Indian

09 $\square$ Pakistani

${ }_{10} \square$ Bangladeshi

${ }_{11} \square$ Any other Asian background, please

specify.

Black or Black British

${ }_{12} \square$ Caribbean

${ }_{13} \square$ African

${ }_{14} \square$ Any other Black background, please

specify.

.................................

Chinese or other ethnic group

${ }_{15} \square$ Chinese

${ }_{16} \square$ Any other background, please

specify.

Please turn to page 4 
10. How old were you when you left full-time education (e.g. school, college or university)?

$$
\text { Age } 16 \text { or less } \square_{1} \quad \text { (Please tick one) }
$$

Age 17-19 $\square_{2}$

Age 20 or over $\square_{3}$

I am still in full-time education $\square_{4}$

11. Are you currently working (either self-employed or in paid employment)?

$$
\begin{array}{lll}
\text { Yes } & \square_{2} \quad \text { (Please tick one) } \\
\text { No } & \square_{1}
\end{array}
$$

12. Are you interested in taking part in this study of back pain?

$$
\begin{array}{lll}
\text { Yes } & \square_{2} \quad \text { (Please tick one) } \\
\text { No } & \square_{1}
\end{array}
$$

13. If you do NOT want to take part in this study, please can you tell us why?

(Please tick as many boxes as apply) I do not want to get just ordinary treatment from my GP $\square_{1}$

I do not want to attend the back skills course $\square_{2}$ Going for treatment will take up too much of my time $\square_{3}$ I do not want to fill in questionnaires $\square_{4}$ I do not want my treatment to be chosen at random $\square_{5}$ I do not want to participate in a group treatment $\square_{6}$ My back pain is not very bad at the moment $\square_{7}$

My back pain is too bad at the moment $\square$ 8

Other (please specify)

14. If you do NOT want us to contact you again about this study, please tick this box. $\square_{1}$

15. We would like to look at the records held at the surgery for some patients with back pain. This is so we can be sure that the people with back pain who take part in the study are similar to those who do not take part. If you are prepared to let us to examine your records, please tick this box

16. If you are interested in taking part in the study, please give your telephone number/s, including the area code and the research nurse will contact you shortly.

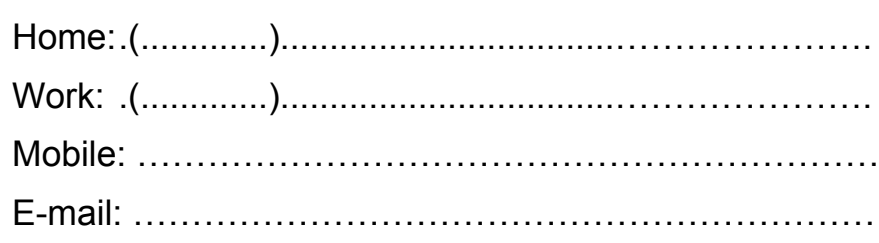

Thank you for filling in the questionnaire. Please return it to the surgery in the envelope provided. 



\section{Appendix 2}

First nurse assessment questionnaire 
BEST/041

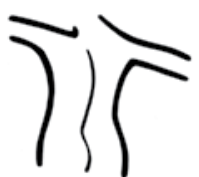

In confidence

Potential participant study ID number

Back Skills Training (BeST) Trial

\section{First nurse assessment form}

The University of Warwick Centre for Primary Health Care
Back Skills Training (BeST) Trial
VERSITY OF WARWICK
MRC General Practice Research Framework

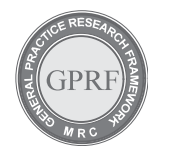


First, please check that the participant study number is correct.

Then ask the potential participant the following questions:

1. On what date are you completing this questionnaire?

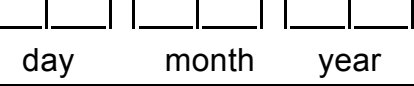

2. What is your date of birth?

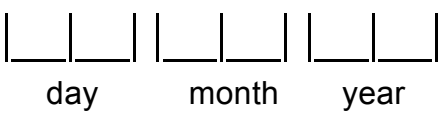

To enter the trial potential participants must be 18 years or older when they attend the randomisation assessment.

First I would like to ask you some questions about your back pain and symptoms.

By back pain we mean pain in the back anywhere between the bottom of the chest and the top of the legs. This area includes the lower back and buttocks. (Show patient laminated card) This is the area marked on this card.

An attack of back pain can last for just a few hours, or may be for much longer. Some people may also experience stiffness, discomfort, anxiety, fatigue, moodiness, numbness or tingling due to the back pain.

\begin{tabular}{|c|c|c|}
\hline & Yes & No \\
\hline 3. Are you still being troubled by your back pain? & & \\
\hline 4. How often have you had back pain or symptoms in the past six weeks? & & \\
\hline Everyday & & \\
\hline Between everyday and three quarters of the days & & \\
\hline Between three quarters of the days and half of the days & & 3 \\
\hline Between half of the days and a quarter of the days & & \\
\hline Rarely & & \\
\hline \multicolumn{3}{|l|}{$\begin{array}{l}\text { 5. How troublesome has your back pain or symptoms been during the past six } \\
\text { weeks? }\end{array}$} \\
\hline Not at all troublesome & & \\
\hline Slightly troublesome & & \\
\hline Moderately troublesome & & \\
\hline Very troublesome & & \\
\hline Extremely troublesome & & \\
\hline
\end{tabular}




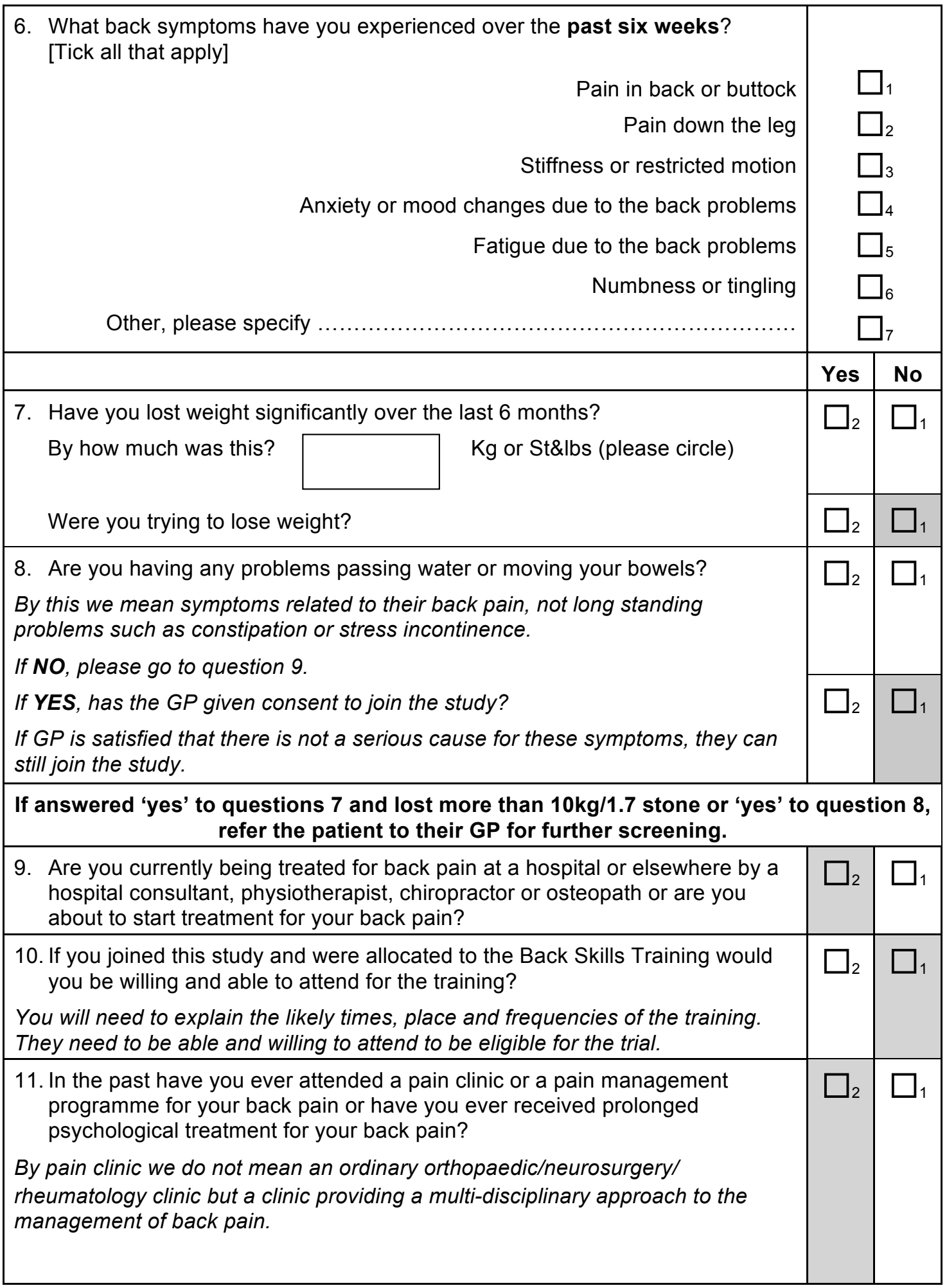




\begin{tabular}{|c|c|c|}
\hline & Yes & No \\
\hline 12. Do you understand the trial? & $\square_{2}$ & $\square_{1}$ \\
\hline 13. Are you interested in taking part in this study of back pain treatments? & $\square_{2}$ & $\square_{1}$ \\
\hline \multicolumn{3}{|l|}{ If YES to Q13 and there are no responses in a shaded box: } \\
\hline \multicolumn{3}{|c|}{$\begin{array}{l}\text { Ask the potential participant to book another appointment to see you at least a week later for } \\
\text { a randomisation assessment. Explain that to enter the study when they see you next they } \\
\text { must have had back pain or symptoms in the few days before they see you. } \\
\text { If they do not think they will be eligible then they should cancel their appointment. They may } \\
\text { book a fresh appointment if their pain becomes more persistent and they want to enter the } \\
\text { study. (tell them when the study is due to end) }\end{array}$} \\
\hline \multicolumn{3}{|c|}{$\begin{array}{l}\text { If NO to Q13: } \\
\text { Explain that this will not affect their care from the practice in any way. However, the } \\
\text { researchers would like to know the reasons why they do not want to take part in the study. }\end{array}$} \\
\hline $\begin{array}{l}\text { 14. Please can you tell why you do not want to take part in the study? [Tick all } \\
\text { that apply] }\end{array}$ & \multirow{2}{*}{\multicolumn{2}{|c|}{$\square_{1}$}} \\
\hline I do not want to get just treatment from my GP & & \\
\hline I do not want to attend the Back Skills Training course & \multicolumn{2}{|c|}{$\square_{2}$} \\
\hline Going for treatment will take up too much of my time & \multicolumn{2}{|c|}{$\square 3$} \\
\hline I do not want to fill in questionnaires & \multicolumn{2}{|c|}{$\square_{4}$} \\
\hline I do not want my treatment to be chosen at random & \multicolumn{2}{|c|}{$\square 5$} \\
\hline I do not want to participate in a group treatment & \multicolumn{2}{|c|}{$\square 6$} \\
\hline My back pain is not very bad at the moment & \multicolumn{2}{|c|}{$\square_{7}$} \\
\hline My back pain is too bad at the moment & \multicolumn{2}{|c|}{$\square_{8}$} \\
\hline 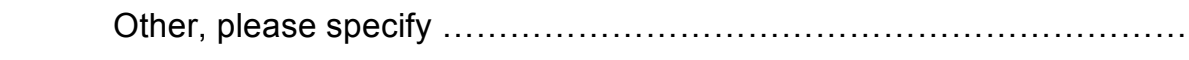 & \multicolumn{2}{|c|}{$\square_{9}$} \\
\hline
\end{tabular}


If the patient appears eligible ensure that the doctors' consent form is completed before their next appointment.

The randomisation assessment should be booked at least one week later, but no longer than four weeks from when the doctor signed the form.

If the only responses in shaded boxes are:

- That their pain is present on less than half of the days (question 4)

- That their pain is not troublesome enough (question 5)

- That they are currently receiving treatment for their back pain or about to start treatment (question 9)

they are not, at present, eligible for the trial. However, if their pain continues and/or they have finished the treatment they can contact you to book a randomisation appointment. Give them a temporary exclusion letter.

\section{Comments}




\section{Appendix 3}

\section{Temporary exclusion letter}


Study number:

Date:

Dear

Thank you for offering to take part in the study of back pain at the surgery. Your help is greatly appreciated. The information you have already given us will help us with our research.

At present you are not eligible for the study. The reason(s) why you are not eligible is/are:

$\square$ Your back pain is present for less than half of the days

$\square$ Your back pain is not at all troublesome or slightly troublesome

$\square$ You are currently receiving treatment or are about the start treatment for your back pain

$\square$ You are not interested to take part in this study or you are unable to attend the treatment sessions

If your back pain continues you may become suitable for the study. You may be eligible if:

- you have back pain on more than half of the days

- your back pain is at least moderately troublesome

- you have finished your back pain treatment

If this happens, we would be most grateful if you would contact us again. Please contact the surgery and ask for an appointment to be assessed for the BeST back pain trial.

Thank you once again

Yours sincerely

Back Skills Training (BeST) Trial

The University of Warwick Centre for Primary Health Care

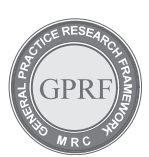

MRC General Practice Research Framework 


\section{Appendix 4 \\ Nurse randomisation assessment questionnaire}


BEST/042

\title{
In confidence
}

\author{
Back Skills Training (BeST) Trial
}

\section{Nurse randomisation assessment form}

Back Skills Training (BeST) Trial

The University of Warwick Centre for Primary Health Care
THE UNIVERSITY OF WARWICK
MRC General Practice Research Framework

A randomised study of treatments for back pain in primary care, funded by the NHS R\&D HTA programme (ISRCTN54717854) 
First, please check that the participant study number is correct. Then ask the potential participant all of the following questions.

Some of these questions are the same as those I asked you last time I saw you.

This is to make sure that you are still suitable for the study.

1. On what date are you completing this form?

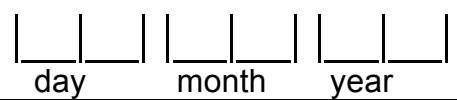

2. What is your date of birth?

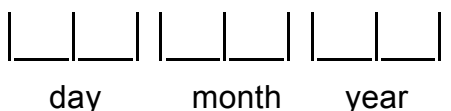

To enter the trial potential participants must be 18 years or older when they attend the randomisation assessment.

First I would like to ask you some questions about your back pain and symptoms.

By back pain we mean pain in the back anywhere between the bottom of the chest and the top of the legs. This area includes the lower back and buttocks. (Show patient laminated card) This is the area marked on this card.

An attack of back pain can last for just a few hours, or may be for much longer. Some people may also experience stiffness, discomfort, anxiety, fatigue, moodiness, numbness or tingling due to the back.

\begin{tabular}{|c|c|c|}
\hline & Yes & No \\
\hline 3. Are you still being troubled by your back pain? & & $\square_{1}$ \\
\hline 4. How often have you had back pain or symptoms in the last six weeks? & & \\
\hline Everyday & & \\
\hline Between everyday and three quarters of the days & & \\
\hline Between three quarters of the days and half of the days & & \\
\hline Between half of the days and a quarter of the days & & \\
\hline Rarely & & \\
\hline $\begin{array}{l}\text { 5. How troublesome has your back pain or symptoms been during the past } 6 \\
\text { weeks? }\end{array}$ & & \\
\hline Not at all troublesome & & \\
\hline Slightly troublesome & & \\
\hline Moderately troublesome & & \\
\hline Very troublesome & & \\
\hline Extremely troublesome & & \\
\hline
\end{tabular}




\begin{tabular}{|c|c|c|c|}
\hline & & Yes & No \\
\hline \multicolumn{2}{|c|}{ 6. Have you lost weight significantly over the last 6 months? } & \multirow{2}{*}{$\square_{2}$} & \\
\hline By how much was this? & \multirow[t]{2}{*}{ Kg or St\&lbs (please circle) } & & \\
\hline Were you trying to lose weight? & & \rfloor$_{2}$ & \\
\hline \multirow{5}{*}{\multicolumn{2}{|c|}{$\begin{array}{l}\text { 7. Are you having any problems passing water or moving your bowels? } \\
\text { By this we mean symptoms related to their back pain, not long standing } \\
\text { problems such as constipation or stress incontinence. } \\
\text { If NO, please go to question } 8 . \\
\text { If YES, has the GP given consent to join the study? } \\
\text { If GP is satisfied that there is not a serious cause for these symptoms, they can } \\
\text { still join the study. }\end{array}$}} & & \\
\hline & & & \\
\hline & & & \\
\hline & & & \\
\hline & & & \\
\hline
\end{tabular}

If answered 'yes' to questions 6 and lost more than $10 \mathrm{~kg} / 1.7$ stone or 'yes' to question 7 , refer the patient to their GP for further screening.

8. Are you currently being treated for back pain at a hospital or elsewhere by a consultant, physiotherapist, chiropractor or osteopath or are you about to start treatment for your back pain?

9. If you joined this trial and were randomised to the Back Skills Training would you be willing and able to attend for treatment?

You will need to explain the likely times, places and frequencies of the training. They need to be able and willing to attend to be eligible for the trial.

10. In the past have you ever attended a specialised pain clinic or pain management programme for back pain or have you ever received prolonged psychological treatment for your back pain?

By pain clinic we do not mean an ordinary orthopaedic/neurosurgery/ rheumatology clinic but a clinic providing a multi-disciplinary approach to the management of back pain.

11. Women only: Are you pregnant at the moment?

If you join the trial and find out that you are pregnant during the first three months please let me know as soon as possible.

Although the back skills training is believed to be safe in early pregnancy we do not want pregnant women to have any trial treatments because a different treatment approach may be needed.

12. Do you want to join this study of back pain treatments?

\section{Check from your records for the following information}

13. Has the GP signed consent for the patient to enter the trial? 
The patient is eligible if there are no responses to questions in a shaded box.

If the patient appears eligible but does not wish to take part, explain that this will not affect their care from the practice in any way. Please ask them their reasons and enter them in the comments section below.

If potential participants are temporarily not eligible for the trial give them a temporary exclusion letter and explain that if they become eligible and they wish to enter the trial then they can book a randomisation appointment with you.

If they are eligible for the trial:

1. ask them to sign the consent form

2. ask them to complete the baseline questionnaire.

After the baseline questionnaire has been completed:

- complete the randomisation form

- randomise the patient

Tell the participant their randomised treatment. If randomised to the Back Skills Training you need to send a notification letter to the therapist. Please advise the patient that the therapist will then contact the participants to arrange a time to start their treatments. Please ensure we have a contact number.

Please give all randomised participants a copy of 'The Back Book'. Reinforce its message and tell them how important it is that they read it and follow its advice. Take time to answer their questions and refer them back to their GP if they still have any queries.

Give the participant:

- An address change form and reply paid envelope to notify address and telephone number changes.

Comments 



\section{Appendix 5 \\ Consent form}


Study Number:

\section{CONSENT FORM}

\section{A research trial into the effectiveness of different treatments for people experiencing low back pain.}

\section{Please initial box}

1. I confirm that I have read and understand the Information sheet dated 2 February 2005 version 5 for the above study and have had the opportunity to ask questions.

2. I understand that my participation is voluntary and that I am free to withdraw at any time, without giving any reason, without my medical care or legal rights being affected.

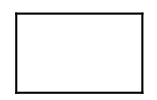

3. I understand that my GP and other health professionals involved in my care will be informed of my participation in the study, and I consent to researchers where it is relevant to have access to my medical records.

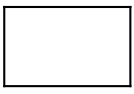

4. I agree to take part in the above study.
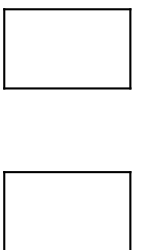

5. I am happy to be invited for an interview during the follow up period of the trial. I understand that if I wish not be interviewed I can still participate in the trial.

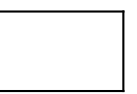

The University of Warwick Centre for Primary Health Care

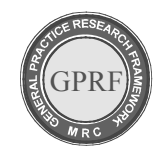

MRC General Practice Research Framework 


\section{Appendix 6}

\section{Baseline questionnaire}


BEST/043

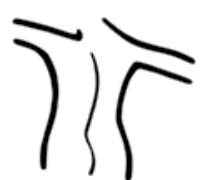

In confidence

Potential participant study ID number

Back Skills Training (BeST) Trial

\section{Baseline Questionnaire}

The University of Warwick

Back Skills Training (BeST) Trial

Centre for Primary Health Care

THE UNIVERSity of

WARWICK

MRC General Practice Research Framework

A randomised study of treatments for back pain in primary care, funded by the NHS R\&D HTA programme (ISRCTN54717854) 


\section{PLEASE READ ALL THE INSTRUCTIONS BEFORE COMPLETING THE QUESTIONNAIRE}

Thank you for agreeing to take part in this study. The responses you give in this questionnaire will help us find out if the treatments you get are helpful for your back problems.

Please answer all the questions. Although it may seem that questions are asked more than once, it is still important that you answer every one.

Please follow the instructions for each section carefully.

For each section, if you are asked to put a cross in the box, please use a cross rather than a tick.

For example in the following question, if your answer to the question is yes, you should place a cross firmly in the box next to yes.

Do you drive a car?

$$
\text { Yes } \bigotimes
$$

No

If you are asked to circle a number, please use a circle rather than underlining a number.

For example, in the following question if you are asked 'how happy are you today?' Where ' 1 ' is 'very unhappy' and ' 5 ' is 'very happy'. If you feel neither happy or unhappy you may wish to answer ' 3 '. You do this by clearly circling the number 3.

2

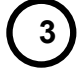

4 5

Please use a BLACK or BLUE pen. Please do not use a pencil. 


\section{Section 1}

This section is to determine the level of back pain and employment status.

Please answer the following questions.

Firstly, please enter the date you are completing this questionnaire:

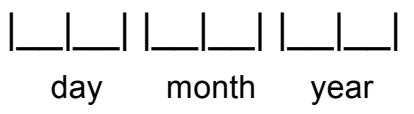

1. How long have you been troubled by back pain? This means when your back pain first ever started.

weeks months years

2. Since your back pain first started which of the following statements describes your back pain? [Tick only one box]

My back pain comes and goes over time
My back pain is fairly constant
My back pain is getting worse
My back pain is getting better

3. Have you received any treatment for your back pain in the past 6 months?

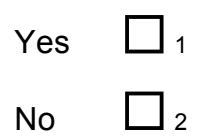

If Yes, please specify

4. Are you currently receiving any benefit payments?

$\begin{array}{lllll} & \text { Yes } & \text { No } & \text { Yes } & \text { No } \\ \text { Statutory Sick Pay } & \square_{1} & \square_{2} & \text { Working Family Tax Credit } & \square_{1} \\ \text { Incapacity Benefit } & \square_{1} & \square_{2} & \text { Council Tax Benefit } & \square_{1} \\ \text { Disability living allowance } & \square_{1} & \square_{2} & \text { Housing Benefit } & \square_{1} \\ \text { Disabled persons tax credit } & \square_{1} & \square_{2} & \text { Attendance allowance } & \square_{1} \\ \text { Invalid Care Allowance } & \square_{1} & \square_{2} \text { (includes if paid to someone who looks after you) } \\ \text { Other } & \square_{1} & \square_{2} \text { (please specify) }\end{array}$


5. Are you currently working? (If you are a full-time student but also work, please complete this section and also tick question 9)
Yes, full time $\square 1$
Yes, part time $\square 2$
No $\square 3$ Please go to question 9

6. Is this work: $\quad$ Paid $\quad \square_{1}$

Unpaid $\quad \square 2$

7. How many hours a week do you work?

$\begin{array}{ll}\text { Less then } 10 & \square 1 \\ 10-25 & \square_{2} \\ 25-40 & \square_{3} \\ \text { More than } 40 & \square_{4}\end{array}$

8. Which of the following categories do you think best describes your work?

$\begin{array}{ll}\text { Unskilled manual } & \square_{1} \\ \text { Skilled manual } & \square_{2} \\ \text { Unskilled non-manual } & \square_{3} \\ \text { Skilled non-manual } & \square_{4} \\ \text { Professional } & \square_{5} \text { Please describe: } \\ \text { Other } & \square_{6} \text { Please describe: }\end{array}$

Decline to answer $\quad \square 7$ 
9. If you are not currently working which of the following applies to you?

Retired
At home and not looking for paid work
(eg looking after home, family or others)
Unable to work due to low back pain
Unable to work due to other illness
Unemployed and looking for work
In full time education
Other

10. What factors are important to you when receiving treatment? [Tick all that apply]

Getting rid of the pain and/or moving about better
Re-assurance that there is nothing serious wrong
Receiving trustworthy information
Having an opportunity to talk to a Dr, Nurse or therapists
Having an opportunity to talk to people with back pain
Someone taking an interest in me
Helping me to manage my back problem

Other:

Which is the most important? : 


\section{Section 2}

This section is about your back pain today. When your back hurts, you may find it difficult to do some of the things you normally do. This list contains some sentences that people have used to describe themselves when they have back pain. When you read them, you may find that some stand out because they describe you today.

As you read the list, think of yourself today. When you read a sentence that describes you today, place a cross in the box beside it. If the sentence does not describe you, then leave the box blank and go on to the next one. Remember, only place a cross if you are sure that it describes you today.

1. I stay at home most of the time because of my back.

2. I change positions frequently to try and get my back comfortable.

3. I walk more slowly than usual because of my back.

4. Because of my back, I am not doing any of the jobs that I usually do around the house.

5. Because of my back, I use a handrail to get upstairs.

6. Because of my back, I lie down to rest more often.

7. Because of my back, I have to hold on to something to get out of an easy chair.

8. Because of my back, I try to get other people to do things for me.

9. I get dressed more slowly than usual because of my back.

10. I only stand up for short periods of time because of my back.

11. Because of my back, I try not to bend or kneel down.

12. I find it difficult to get out of a chair because of my back.

13. My back is painful almost all the time.

14. I find it difficult to turn over in bed because of my back.

15. My appetite is not very good because of my back pain.

16. I have trouble putting on my socks (or stockings) because of the pain in my back.

17. I only walk short distances because of my back pain.

18. I sleep less well because of my back.

19. Because of my back pain, I get dressed with help from someone else.

20. I sit down for most of the day because of my back.

21. I avoid heavy jobs around the house because of my back.

22. Because of my back pain, I am more irritable and bad tempered with people than usual.

23. Because of my back, I go upstairs more slowly than usual.

24. I stay in bed most of the time because of my back.

25 . None of the above. 


\section{Section 3}

This section is about how much your back trouble has been interfering with your daily activities in recent weeks.

For the next six questions please circle the number which represents how your back pain has made you feel over the last 4 weeks.

1. In the past $\mathbf{4}$ weeks, how much has your back pain interfered with your daily activities on a scale of $0-10$ where 0 is 'no interference' and 10 is 'unable to carry out any activities at all'?

$$
\begin{array}{lllllllllll}
0 & 1 & 2 & 3 & 4 & 5 & 6 & 7 & 8 & 9 & 10
\end{array}
$$

2. In the past $\mathbf{4}$ weeks, how much has your back pain changed your ability to take part in recreational, social and family activities on a scale of $0-10$ where $\underline{0 \text { is 'no change' and } 10 \text { is }}$ 'extreme change'?

$$
\begin{array}{lllllllllll}
0 & 1 & 2 & 3 & 4 & 5 & 6 & 7 & 8 & 9 & 10
\end{array}
$$

3. In the past $\mathbf{4}$ weeks, how much has your back pain changed your ability to work (including housework) on a scale of $0-10$ where 0 is 'no change' and 10 is 'extreme change'?

$\begin{array}{lllllllllll}0 & 1 & 2 & 3 & 4 & 5 & 6 & 7 & 8 & 9 & 10\end{array}$

4. In the past $\mathbf{4}$ weeks, how bad has your worst back pain been on a scale of $0-10$ where $\underline{0 \text { is 'no }}$ pain' and 10 is 'as bad as a pain could be'?

$\begin{array}{lllllllllll}0 & 1 & 2 & 3 & 4 & 5 & 6 & 7 & 8 & 9 & 10\end{array}$

5. In the past 4 weeks, on average how bad has your back pain been on a scale of 0-10 where $\underline{0}$ is 'no pain' and 10 is 'as bad as a pain could be'?

$\begin{array}{lllllllllll}0 & 1 & 2 & 3 & 4 & 5 & 6 & 7 & 8 & 9 & 10\end{array}$

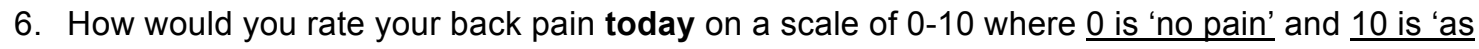
bad as a pain could be'.

$\begin{array}{lllllllllll}0 & 1 & 2 & 3 & 4 & 5 & 6 & 7 & 8 & 9 & 10\end{array}$




\section{Section 4}

This section is to determine what you believe about back pain. Here are some of the things which other patients have told us about their pain. For each statement please circle the number from 0 to 6 to say how much physical activities such as bending lifting walking or driving affect or would affect your back pain.

Please circle one number for each line

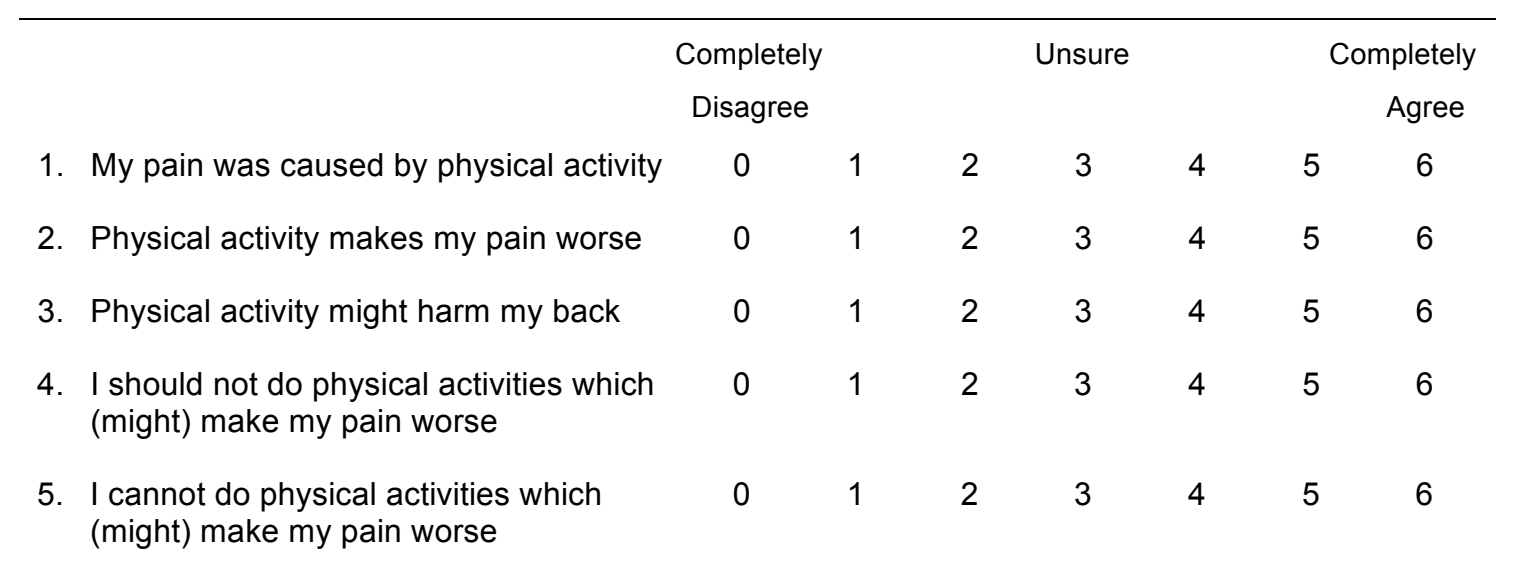

\section{Section 5}

This section is to determine how much your low back pain has troubled you lately.

1. During the past $\mathbf{4}$ weeks, about how many days did low back pain keep you from going to work or school/college/university? days

2. During the past 4 weeks, about how many days did you have to cut down on the things you usually do for more than half the day because of back pain? days 


\section{Section 6}

This questionnaire asks for your views about your health. This information will help keep track of how you feel and how well you are able to do your usual activities.

For each of the following questions, please place a cross in the one box that best describes your answer.

1. In general, would you say your health is:

Excellent

Very good

Good

Fair

Poor
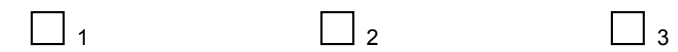

$\square_{4}$

2. The following questions are about activities you might do during a typical day. Does your health now limit you in these activities? If so, how much?

$\begin{array}{ccc}\begin{array}{c}\text { Yes, } \\ \text { limited } \\ \text { a lot }\end{array} & \begin{array}{c}\text { Yes, } \\ \text { limited } \\ \text { a little }\end{array} & \begin{array}{c}\text { No, not } \\ \text { limited } \\ \text { at all }\end{array} \\ \square_{1} & \square_{2} & \square_{3} \\ \square_{1} & \square_{2} & \square_{3}\end{array}$

3. During the past 4 weeks, how much of the time have you had any of the following problems with your work or other regular daily activities as a result of your physical health?

$\begin{array}{ccccc}\text { All of } & \text { Most of } & \text { Some of } & \text { A little of } & \text { None of } \\ \text { the time } & \text { the time } & \text { the time } & \text { the time }\end{array}$

a) Accomplished less than you would like

b) Were limited in the kind of work or other activities 
4. During the past $\mathbf{4}$ weeks, how much of the time have you had any of the following problems with your work or other regular activities as a result of any emotional problems (such as feeling depressed or anxious)?

$\begin{array}{ccccc}\begin{array}{c}\text { All of } \\ \text { the time }\end{array} & \begin{array}{c}\text { Most of } \\ \text { the time }\end{array} & \begin{array}{c}\text { Some of } \\ \text { the time }\end{array} & \begin{array}{c}\text { A little of } \\ \text { the time }\end{array} & \begin{array}{c}\text { None of } \\ \text { the time }\end{array} \\ \square_{1} & \square_{2} & \square_{3} & \square_{4} & \square{ }_{5} \\ \square_{1} & \square_{2} & \square_{3} & \square_{4} & \square_{5}\end{array}$

5. During the past 4 weeks, how much did pain interfere with your normal work (including both work outside the home and housework)?

Not at all A little bit Moderately Quite a bit Extremely

Accomplished less than you would like

b) Were limited in the kind of work or other activities

$\begin{array}{ccccc}\text { Not at all } & \text { A little bit } & \text { Moderately } & \text { Quite a bit } & \text { Extremely } \\ \square_{1} & \square_{2} & \square_{3} & \square_{4} & \square_{5}\end{array}$

6. These questions are about how you feel and how things have been with you during the past 4 weeks. For each question, please give the one answer that comes closest to the way you have been feeling. How much of the time during the past 4 weeks:

$\begin{array}{ccccc}\text { All of the } & \begin{array}{c}\text { Most of } \\ \text { time }\end{array} \text { the time } & \begin{array}{c}\text { Some of } \\ \text { the time }\end{array} & \begin{array}{c}\text { A little of } \\ \text { the time }\end{array} & \begin{array}{c}\text { None of } \\ \text { the time }\end{array}\end{array}$

a) Have you felt calm and peaceful?
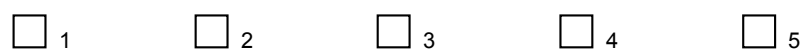

b) Did you have a lot of energy?
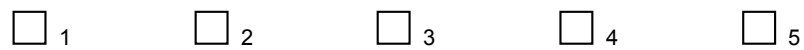

c) Have you felt downhearted and low?
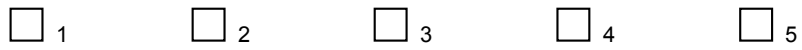

7. During the past $\mathbf{4}$ weeks, how much of the time has your physical health OR emotional problems interfered with your social activities (like visiting with friends, relatives, etc.)?

$\begin{array}{ccccc}\text { All of } & \text { Most of } & \text { Some of } & \text { A little of } & \text { None of } \\ \text { the time } & \text { the time } & \text { the time } & \text { the time } & \text { the time } \\ \square_{1} & \square_{2} & \square_{3} & \square_{5} & \square_{5}\end{array}$




\section{Section 7}

The following questions are to ask about your general health state at the moment. By placing a tick (' $\checkmark$ ') in one box in each group below, please indicate which statement best describes your own health state today.

Do not tick more than one box per question.

\section{Mobility:}

I have no problems in walking about

I have some problems in walking about

I am confined to bed

\section{Self-Care:}

I have no problems with self-care

I have some problems washing or dressing myself

I am unable to wash or dress myself

3. Usual Activities (e.g. work, study, housework, family or leisure activities):

I have no problems with performing my usual activities

I have some problems with performing my usual activities

I am unable to perform my usual activities

\section{Pain / Discomfort:}

I have no pain or discomfort

I have moderate pain or discomfort

I have extreme pain or discomfort

\section{Anxiety / Depression:}

I am not anxious or depressed

I am moderately anxious or depressed

I am extremely anxious or depressed 


\section{Your own health state today}

To help people say how good or bad a health state is, we have drawn a scale (rather like a thermometer) on which the best state you can imagine is marked by 100 and the worst state you can imagine is marked by 0 .

We would like you to indicate on this scale how good or bad is your own health today, in your opinion.

Please do this by drawing a line from the box below, to whichever point on the scale indicates how good or bad your current health state is today.

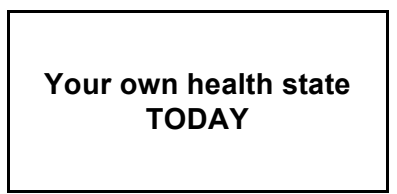

Bcst

imaginable
health state

100

主

莑

金。

事

染

Worst

imaginable

health state 


\section{Section 8}

This section is about how you are feeling at the moment. Please place a cross in the box that comes closest to how you have been feeling in the past week.

Do not take too long over your replies: your immediate reaction to each item will probably be more accurate than a long thought out response.

1. I feel tense or 'wound up':

Most of the time

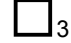

A lot of the time

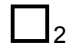

From time to time, occasionally

Not at all

$\square$

3. I get a sort of frightened feeling as if something awful is about to happen:

Very definitely and quite badly

$\square_{3}$

Yes, but not too badly

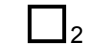

A little, but it doesn't worry me

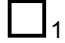

Not at all

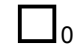

5. Worrying thoughts go through my mind:

A great deal of the time

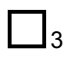

A lot of the time

$\square_{2}$

From time to time but not too often $\square$

Only occasionally

7. I can sit at ease and feel relaxed:

Definitely

$\square 0$

Usually

$\square 1$

Not often

$\square 2$

Not at all
2. I still enjoy the things I used to enjoy:

Definitely as much

Not quite as much

Only a little

Hardly at all

4. I can laugh and see the funny side of things:

As much as I always could

$\square_{0}$

Not quite as much now

Definitely not so much now

Not at all

6. I feel cheerful:

Not at all<smiles>C1CCC1</smiles>

Not often<smiles>C1CCC1</smiles>

Sometimes

Most of the time

8. I feel as if I am slowed down:

Nearly all the time

Very often

Sometimes

Not at all 
9. I get a sort of frightened feeling like 'butterflies' In the stomach:

Not at all<smiles>C1CCC1</smiles>

Occasionally<smiles>C1C[AlH2]C1</smiles>

Quite often

Very often

$\square_{3}$

11. I feel restless as if I have to be on the move:

Very much indeed

$\square 3$

Quite a lot

$\square 2$

Not very much

$\square 1$

Not at all

$\square 0$

13. I get sudden feeling of panic:

Very often indeed

$\square 3$

Quite often

$\square 2$

Not very often

$\square_{1}$

Not at all
10. I have lost interest in my appearance:

Definitely

I don't take as much care as I should

I may not take quite as much care

I take just as much care as ever

$\square 3$

$\square 2$

12. I look forward with enjoyment to things:

As much as I ever did

Rather less than I used to

Definitely less than I used to

Hardly at all

14. I can enjoy a good book or radio or TV programme:

Often

Sometimes

Not often

Very seldom 


\section{Section 9}

Please rate how confident you are that you can do the following things at present, despite the pain. To answer circle one of the numbers on the scale under each item, where $0=$ 'not at all confident' and $\mathbf{6}=$ 'completely confident'.

For example

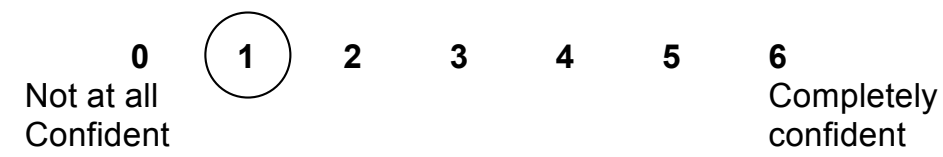

Remember, this questionnaire is not asking whether or not you have been doing these things, but rather how confident you are that you can do them at present, despite the pain.

$$
\begin{aligned}
& \text { Not at all } \\
& \text { Confident }
\end{aligned}
$$

1. I can enjoy things, despite the pain

$\begin{array}{lllllll}0 & 1 & 2 & 3 & 4 & 5 & 6 \\ 0 & 1 & 2 & 3 & 4 & 5 & 6\end{array}$

2. I can do most of the household chores (e.g. tidying-up, washing dishes, etc.), despite the pain

3. I can socialise with my friends or family members as often as I used to do, despite the pain

4. I can cope with my pain in most situations

5. I can do some form of work, despite the pain. ('work' includes housework, paid and unpaid work).

6. I can still do many of the things I enjoy doing, such as hobbies or leisure activity, despite the pain

7. I can cope with my pain without medication

8. I can still accomplish most of my goals in life, despite the pain

9. I can live a normal lifestyle, despite the pain

$\begin{array}{lllllll}0 & 1 & 2 & 3 & 4 & 5 & 6 \\ 0 & 1 & 2 & 3 & 4 & 5 & 6\end{array}$

I can gradually become more active, despite the pain 


\section{THANK YOU FOR COMPLETING THIS QUESTIONNAIRE}

If you have any general comments about your back pain, or this questionnaire, please write them overleaf. 
Appendix 6

Your comments: 


\section{Appendix 7}

\section{Randomisation form}




\section{RANDOMISATION FORM}

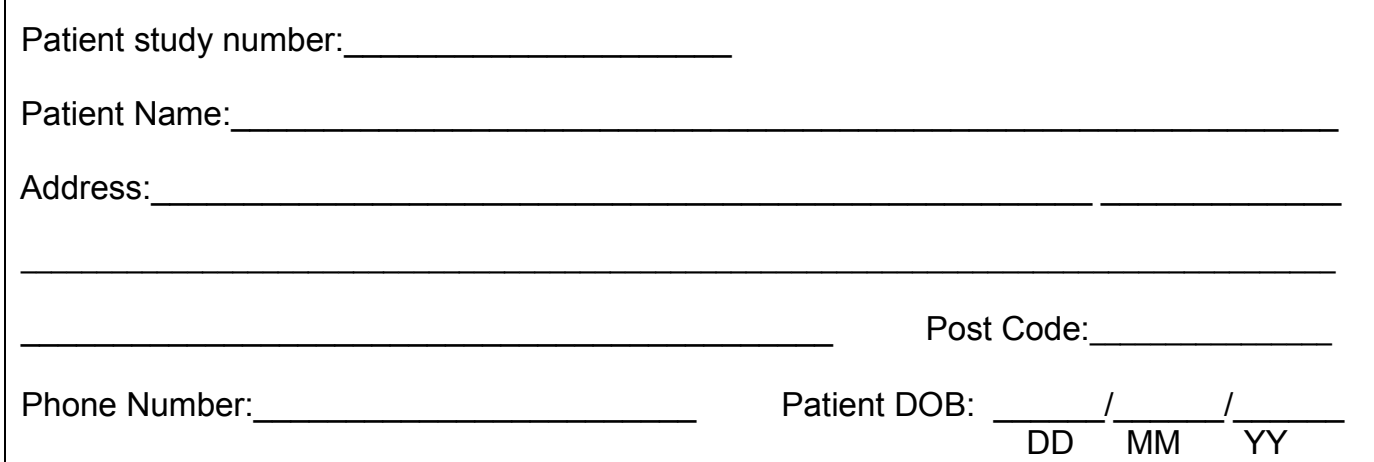

Inclusion criteria Please tick:

\begin{tabular}{|l|l|l|}
\cline { 2 - 3 } \multicolumn{1}{l|}{ 1. Aged 18 and older? } & Yes & No \\
\hline 2. Has low back pain lasted for at least 6 weeks? & & \\
\hline 3. Patient reports at least moderately troublesome LBP? & & \\
\hline
\end{tabular}

\section{If any shaded box is ticked, the patient is not eligible}

\section{Randomisation information}

1. GP Practice or triage service:

2. How troublesome is your back pain been during the past week?

Moderately troublesome

Very/extremely troublesome

\section{Treatment allocation}

The patient has been allocated to receive:
A: Active management
B: Active management + cognitive behavioural approach

Name

Randomisation date:

Signed:

$\mathrm{DD} / \mathrm{M} \overline{\mathrm{M}} \mathrm{Y} \mathrm{Y}$

Please send this form via special delivery to the co-ordinating centre 


\section{Appendix 8}

\section{Follow-up questionnaires}


In confidence

Potential participant study ID number

Back Skills Training (BeST) Trial

Trial Participant Three Month Follow Up Questionnaire 


\section{PLEASE READ ALL THE INSTRUCTIONS BEFORE COMPLETING THE QUESTIONNAIRE}

Thank you for agreeing to take part in this study. The responses you give in this questionnaire will help us find out if the advice or treatment you have had for your back as part of the study has been helpful.

Please answer all the questions. Although it may seem that questions are asked more than once, it is still important that you answer every one.

Please follow the instructions for each section carefully.

For example in the following question, if your answer to the question is yes, you should place a cross firmly in the box next to yes.

Do you drive a car?

No

If you are asked to circle a number, please use a circle rather than underlining a number.

For example, in the following question if you are asked 'how happy are you today?' Where ' 1 ' is 'very unhappy' and ' 5 ' is 'very happy'. If you feel neither happy or unhappy you may wish to answer ' 3 '. You do this by clearly circling the number 3 .
1
2

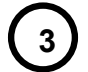
4
5

Please use a BLACK or BLUE pen. Please do not use a pencil.

All the responses you give will be kept strictly confidential. 
Firstly, please enter the date you are completing this questionnaire:

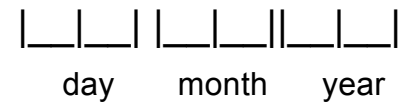

\section{Section 1}

By placing a cross (' $X$ ') in one box for each question below, please indicate which statement best describes your feelings towards the advice or treatment you have received for your lower back pain as part of the study.

1. How satisfied are you with the advice or treatment you received?

$\begin{array}{ll}\text { Very dissatisfied } & \square \\ \text { Somewhat dissatisfied } & \square_{2} \\ \text { Neither satisfied nor dissatisfied } & \square_{3} \\ \text { Somewhat satisfied } & \square_{4} \\ \text { Very satisfied } & \square_{5}\end{array}$

2. How much benefit have you gained from the advice or treatment you have received for your lower back pain as part of the study.

$\begin{array}{ll}\text { Substantial benefit } & \square \text { 1 } \\ \text { Moderate benefit } & \square_{2} \\ \text { No benefit } & \square_{3} \\ \text { Soderate harm } & \square_{5}\end{array}$




\section{Section 2}

When your back hurts, you may find it difficult to do some of the things you normally do. This list contains some sentences that people have used to describe themselves when they have back pain. When you read them, you may find that some stand out because they describe you today.

As you read the list, think of yourself today. When you read a sentence that describes you today, place a cross in the box beside it. If the sentence does not describe you, then leave the box blank and go on to the next one. Remember, only place a cross if you are sure that it describes you today.

1. I stay at home most of the time because of my back.

2. I change positions frequently to try and get my back comfortable.

3. I walk more slowly than usual because of my back.

4. Because of my back, I am not doing any of the jobs that I usually do around the house.

5. Because of my back, I use a handrail to get upstairs.

6. Because of my back, I lie down to rest more often.

7. Because of my back, I have to hold on to something to get out of an easy chair.

8. Because of my back, I try to get other people to do things for me.

9. I get dressed more slowly than usual because of my back.

10. I only stand up for short periods of time because of my back.

11. Because of my back, I try not to bend or kneel down.

12. I find it difficult to get out of a chair because of my back.

13. My back is painful almost all the time.

14. I find it difficult to turn over in bed because of my back.

15. My appetite is not very good because of my back pain.

16. I have trouble putting on my socks (or stockings) because of the pain in my back.

17. I only walk short distances because of my back pain.

18. I sleep less well because of my back.

19. Because of my back pain, I get dressed with help from someone else.

20. I sit down for most of the day because of my back.

21. I avoid heavy jobs around the house because of my back.

22. Because of my back pain, I am more irritable and bad tempered with people than usual.

23. Because of my back, I go upstairs more slowly than usual.

24. I stay in bed most of the time because of my back. 


\section{Section 3}

This section is about how much your back trouble has been interfering with your daily activities in recent weeks.

For the next six questions please circle the number which represents how your back pain has made you feel over the last 4 weeks.

For example:
0
1
2
4

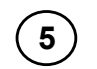
6
$\begin{array}{lll}7 & 8 & 9\end{array}$
10

1. In the past $\mathbf{4}$ weeks, how much has your back pain interfered with your daily activities on a

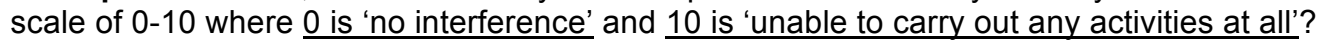

$\begin{array}{lllllllllll}0 & 1 & 2 & 3 & 4 & 5 & 6 & 7 & 8 & 9 & 10\end{array}$

2. In the past $\mathbf{4}$ weeks, how much has your back pain changed your ability to take part in recreational, social and family activities on a scale of $0-10$ where $\underline{0 \text { is 'no change' and } 10 \text { is }}$ 'extreme change'?

$\begin{array}{lllllllllll}0 & 1 & 2 & 3 & 4 & 5 & 6 & 7 & 8 & 9 & 10\end{array}$

3. In the past $\mathbf{4}$ weeks, how much has your back pain changed your ability to work (including housework) on a scale of $0-10$ where 0 is 'no change' and 10 is 'extreme change'?

$\begin{array}{lllllllllll}0 & 1 & 2 & 3 & 4 & 5 & 6 & 7 & 8 & 9 & 10\end{array}$

4. In the past $\mathbf{4}$ weeks, how bad has your worst back pain been on a scale of $0-10$ where $\underline{0 \text { is }}$ 'no pain' and 10 is 'as bad as a pain could be'?

$\begin{array}{lllllllllll}0 & 1 & 2 & 3 & 4 & 5 & 6 & 7 & 8 & 9 & 10\end{array}$

5. In the past 4 weeks, on average how bad has your back pain been on a scale of $0-10$

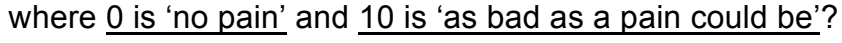

$\begin{array}{lllllllllll}0 & 1 & 2 & 3 & 4 & 5 & 6 & 7 & 8 & 9 & 10\end{array}$

6. How would you rate your back pain today on a scale of $0-10$ where $\underline{0 \text { is 'no pain' and } 10 \text { is }}$ 'as bad as a pain could be'?

$\begin{array}{lllllllllll}0 & 1 & 2 & 3 & 4 & 5 & 6 & 7 & 8 & 9 & 10\end{array}$




\section{Section 4}

Please rate how confident you are that you can do the following things at present, despite the pain. To answer circle one of the numbers on the scale under each item, where $0=$ 'not at all confident' and $6=$ 'completely confident'.

For example

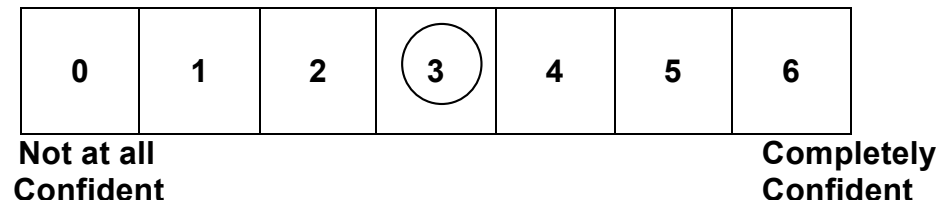

Remember, this questionnaire is not asking whether or not you have been doing these things, but rather how confident you are that you can do them at present, despite the pain.

$\begin{array}{lr}\text { Not at all } & \text { Completely } \\ \text { Confident } & \text { Confident }\end{array}$

1. I can enjoy things, despite the pain.

2. I can do most of the household chores (e.g. tidying-up, washing dishes, etc.), despite the pain.

3. I can socialise with my friends or family members as often as I used to do, despite the pain.

4. I can cope with my pain in most situations.

5. I can do some form of work, despite the pain ('work' includes housework, paid and unpaid work).

6. I can still do many of the things I enjoy doing, such as hobbies or leisure activity, despite the pain.

7. I can cope with my pain without medication.

8. I can still accomplish most of my goals in life, despite the pain.

9. I can live a normal lifestyle, despite the pain.

10. I can gradually become more active, despite the pain.

\begin{tabular}{|l|l|l|l|l|l|l|}
\hline 0 & 1 & 2 & 3 & 4 & 5 & 6 \\
\hline 0 & 1 & 2 & 3 & 4 & 5 & 6 \\
\hline 0 & 1 & 2 & 3 & 4 & 5 & 6 \\
\hline 0 & 1 & 2 & 3 & 4 & 5 & 6 \\
\hline 0 & 1 & 2 & 3 & 4 & 5 & 6 \\
\hline 0 & 1 & 2 & 3 & 4 & 5 & 6 \\
\hline 0 & 1 & 2 & 3 & 4 & 5 & 6 \\
\hline 0 & 1 & 2 & 3 & 4 & 5 & 6 \\
\hline 0 & 1 & 2 & 3 & 4 & 5 & 6 \\
\hline
\end{tabular}




\section{Section 5}

These are some things people have told us about their back pain. For each statement please circle a number from 0 to 6 to say how much physical activity such as bending, lifting, or driving affects your pain.

Please circle one number for each line

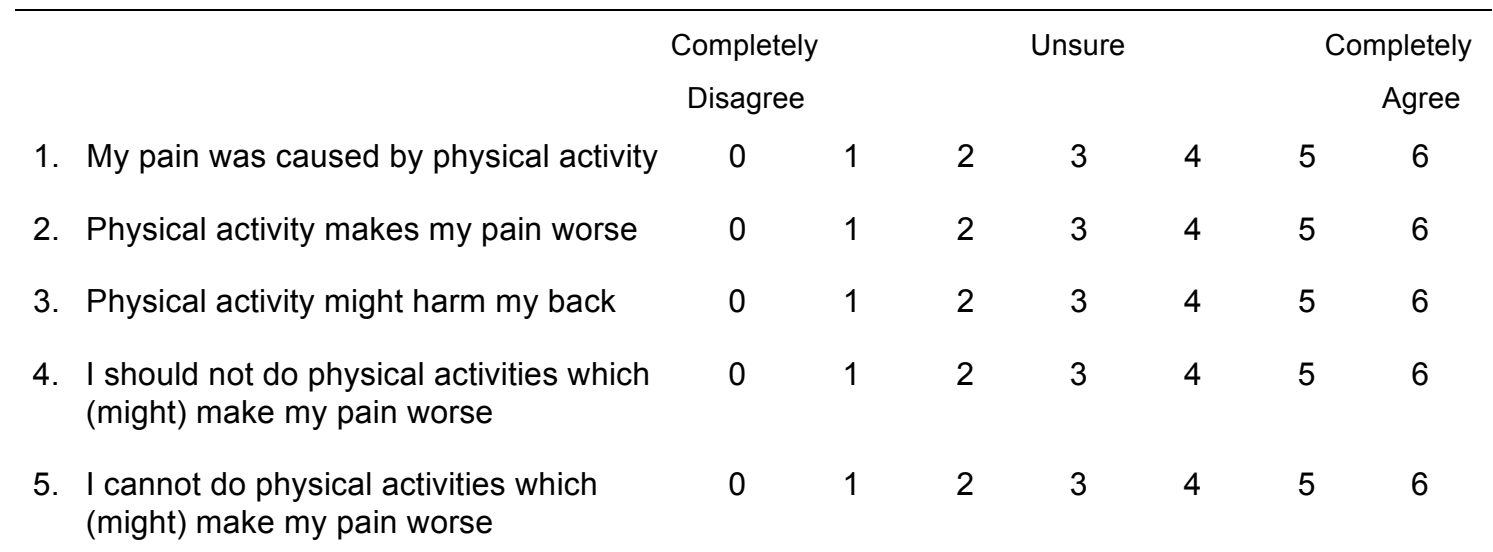

\section{Section 6}

This section is to determine how much your low back pain has changed since you started the study.

1. In what way has your back pain changed in the past three months?

(Please cross one box)

Completely recovered

Much improved

Slightly improved

No change

Slightly worsened

Much worsened

Vastly worsened 


\section{Section 7}

This section asks for your views about your health. This information will help keep track of how you feel and how well you are able to do your usual activities.

For each of the following questions, please place a cross in the one box that best describes your answer.

1. In general, would you say your health is:

Excellent

Very good

Good

Fair

Poor

$\square 1$

$\square_{2}$

3

$\square_{4}$

5

2. The following questions are about activities you might do during a typical day.

Does your health now limit you in these activities? If so, how much?

$\begin{array}{ccc}\begin{array}{c}\text { Yes, } \\ \text { limited } \\ \text { a lot }\end{array} & \begin{array}{c}\text { Yes, } \\ \text { limited } \\ \text { a little }\end{array} & \begin{array}{c}\text { No, not } \\ \text { limited } \\ \text { at all }\end{array} \\ \square_{1} & \square_{2} & \square_{3} \\ \square \square_{1} & \square_{2} & \square_{3}\end{array}$

a) Moderate activities, such as moving a table, pushing a vacuum cleaner, bowling or playing golf

b) Climbing several flights of stairs

3. During the past $\mathbf{4}$ weeks, how much of the time have you had any of the following problems with your work or other regular daily activities as a result of your physical health?

$\begin{array}{ccccc}\begin{array}{c}\text { All of } \\ \text { the time }\end{array} & \begin{array}{c}\text { Most of } \\ \text { the time }\end{array} & \begin{array}{c}\text { Some of } \\ \text { the time }\end{array} & \begin{array}{c}\text { A little of } \\ \text { the time }\end{array} & \begin{array}{c}\text { None of } \\ \text { the time }\end{array}\end{array}$

a) Accomplished less than you would like

$\begin{array}{lllll}\square_{1} & \square_{2} & \square_{3} & \square_{4} & \square 5 \\ \square_{1} & \square_{2} & \square_{3} & \square_{4} & \square_{5}\end{array}$


4. During the past $\mathbf{4}$ weeks, how much of the time have you had any of the following problems with your work or other regular activities as a result of any emotional problems (such as feeling depressed or anxious)?

$\begin{array}{ccccc}\begin{array}{c}\text { All of } \\ \text { the time }\end{array} & \begin{array}{c}\text { Most of } \\ \text { the time }\end{array} & \begin{array}{c}\text { Some of } \\ \text { the time }\end{array} & \begin{array}{c}\text { A little of } \\ \text { the time }\end{array} & \begin{array}{c}\text { None of } \\ \text { the time }\end{array} \\ \square_{1} & \square_{2} & \square_{3} & \square_{4} & \square \square_{5} \\ \square_{1} & \square_{2} & \square_{3} & \square_{4} & \square_{5}\end{array}$

5. During the past 4 weeks, how much did pain interfere with your normal work (including both work outside the home and housework)?

a) Accomplished less than you would like

b) Were limited in the kind of work or other activities

$\begin{array}{ccccc}\text { Not at all } & \text { A little bit } & \text { Moderately } & \text { Quite a bit } & \text { Extremely } \\ \square_{1} & \square_{2} & \square_{3} & \square_{4} & \square 5\end{array}$

6. These questions are about how you feel and how things have been with you during the past 4 weeks. For each question, please give the one answer that comes closest to the way you have been feeling. How much of the time during the past 4 weeks:

a) Have you felt calm and peaceful? time the time

Some of

A little of

None of

the time

the time

the time

b) Did you have a lot of energy?
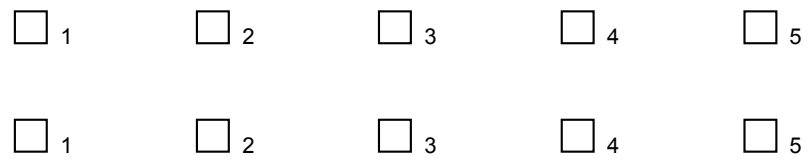

c) Have you felt downhearted and low?

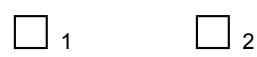

$\square 3$ 5

7. During the past $\mathbf{4}$ weeks, how much of the time has your physical health OR emotional problems interfered with your social activities (like visiting with friends, relatives, etc.)?

$\begin{array}{ccccc}\text { All of } & \text { Most of } & \text { Some of } & \text { A little of } & \text { None of } \\ \text { the time } & \text { the time } & \text { the time } & \text { the time } & \text { the time } \\ \square_{1} & \square_{2} & \square_{3} & \square_{5} & \square_{5}\end{array}$




\section{$\underline{\text { Section } 8}$}

The following questions are to ask about your general health state at the moment. By placing a cross (' $X$ ') in one box in each group below, please indicate which statement best describes your own health state today.

Do not cross more than one box per question.

\section{Mobility:}

I have no problems in walking about

I have some problems in walking about

I am confined to bed

2. Self-Care:

I have no problems with self-care

I have some problems washing or dressing myself

I am unable to wash or dress myself

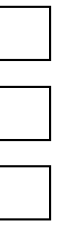

I have no problems with performing my usual activities

I have some problems with performing my usual activities

I am unable to perform my usual activities

4. Pain / Discomfort:

I have no pain or discomfort

I have moderate pain or discomfort

I have extreme pain or discomfort

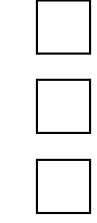

\section{Anxiety / Depression:}

I am not anxious or depressed

I am moderately anxious or depressed

I am extremely anxious or depressed 


\section{Your own health state today}

To help people say how good or Best imaginable bad a health state is, we have drawn a scale (rather like a thermometer) on which the best state you can imagine is marked by 100 and the worst state you can imagine is marked by 0 .

We would like you to indicate on this scale how good or bad is your own health today, in your opinion.

Please do this by drawing a line from the box below, to whichever point on the scale indicates how good or bad your current health state is today.

Your own health state TODAY

100

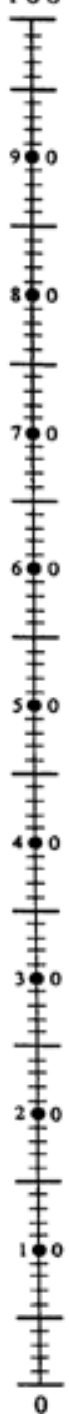

Worst

imaginablo

hearth state 


\section{Section 9}

This section is about health care you have received for your back pain. There are separate parts for NHS treatment, private treatment, products/equipment, normal activities and any benefits/entitlements. Please read each question carefully. For each question, if you have had no treatments or visits, please enter ' 0 '.

\section{NHS Treatment}

1. In the last $\mathbf{3}$ months, how often have you attended the following NHS services for lower back pain? (Please do not include any sessions or treatments that you attended as part of the study).

Number of times

Your GP or another GP

Practice nurse

Physiotherapist

Doctor/nurse in an accident and emergency department (Casualty)

Hospital specialist (consultant or one of his/her team)

Psychologist/Counsellor

Other (please specify)

Other (please specify)
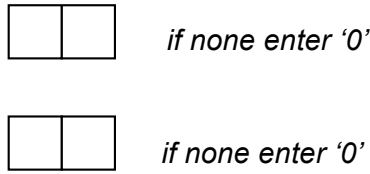

if none enter ' 0 '

if none enter ' 0 '
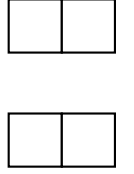

if none enter ' 0 '

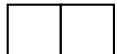

if none enter ' 0 '

if none enter ' 0 '

if none enter ' 0 '

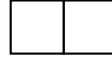

if none enter ' 0 '

2. In the last $\mathbf{3}$ months have you been admitted to an NHS hospital because of back pain?

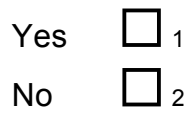

If Yes, in total, how many days were you in hospital? 
3. In the last $\mathbf{3}$ months have you had any of the following tests in a NHS hospital in relation to lower back pain?

Number of times

X-ray

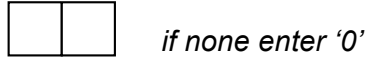

CT Scan

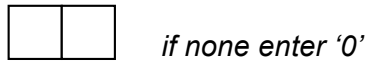

MRI Scan

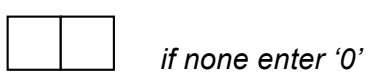

Blood tests (count all blood tests done on one day, as one test)

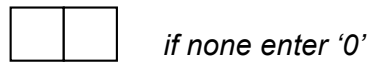

Other (please specify)

if none enter ' 0 '

4. In the last $\mathbf{3}$ months has your doctor prescribed any of the following medications for your back?

Pain killers

Anti-inflammatory drugs (for example ibuprofen, naproxen)

Gels/Creams (for example ibuleve or movelat)

Sleeping pills

Anti-depressants

Other (please specify)

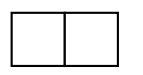

if none enter ' 0 '

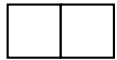

if none enter ' 0 '

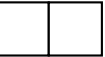

if none enter ' 0 '

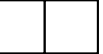

if none enter ' 0 '

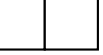

if none enter ' 0 '

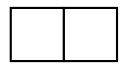

if none enter ' 0 '

5. Do you qualify for free prescriptions?

$\begin{array}{ll}\text { Yes } & \square_{1} \\ \text { No } & \square_{2}\end{array}$




\section{Private Treatment}

6. For the last $\mathbf{3}$ months please detail total treatment costs you paid for yourself; or paid for by private insurance; please do not include any treatments paid for by the NHS. Please round the amounts to the nearest pound. If none enter ' 0 '

$\begin{array}{lll}\text { Number } & \text { Medical } & \text { Personal } \\ \text { Of times } & \begin{array}{l}\text { Insurance } \\ \text { Contribution }\end{array} & \text { Contribution }\end{array}$

Physiotherapist

Hospital specialist (consultant)

Osteopath

Chiropractor

Psychologist

Counsellor

Massage therapist

Aromatherapist

Acupuncturist

Other (please specify)

Other (please specify)

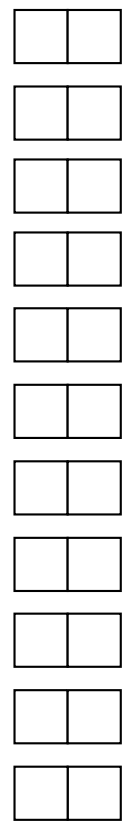

$£$

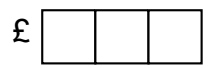

$£$

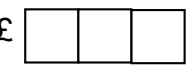

$£$

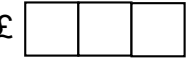

$£$

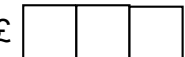

$£$

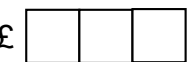

$£$

$£$

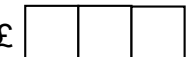

$£$

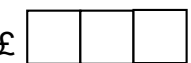

$£$

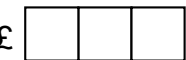

$£$

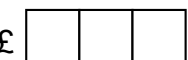

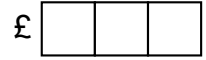
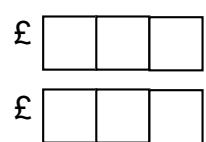

$£$
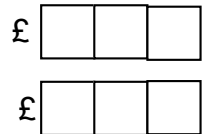

$£$
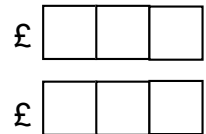

$£$

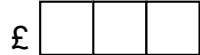

7. In the last $\mathbf{3}$ months, have you been admitted to a private hospital because of lower back pain?

$\begin{array}{ll}\text { Yes } & \square 1 \\ \text { No } & \square_{2}\end{array}$

If Yes, in total, how many days were you in hospital?

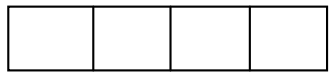

If Yes, what were the total costs paid by your medical insurance? $£$

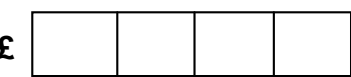

Please give the total costs to the nearest pound.*

If Yes, what were the total costs paid by you?

$£$

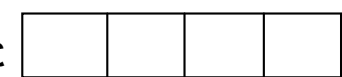

Please give the total costs to the nearest pound.*

*If you do not know the actual cost please give us your best estimate of the costs. 
8. In the last $\mathbf{3}$ months, have you had any of the following tests in a private hospital in relation to lower back pain?

Number of times

X-ray

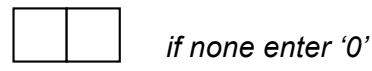

CT Scan

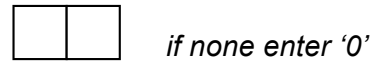

MRI Scan

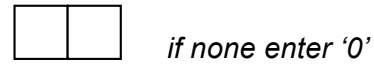

Blood tests (please count all tests done on one day, as one test)

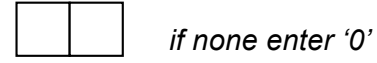

Other (please specify)

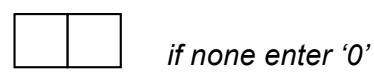

9. In the last $\mathbf{3}$ months have you bought (other than by a prescription) any of the following treatments for your back pain? Please estimate the total cost to the nearest pound.

Pain killers (for example paracetamol/anadin)
Anti-inflammatory drugs (for example ibuprofen/neurofen)
Gels/Creams (for example ibuleve or movelat)
Other (please specify)
Other (please specify)




\section{Products / equipment.}

10. In the last three months, have you bought items such as braces or aids, a new bed or mattress, a chair, a massage machine, or any other products or equipment because of your back pain? (please list the item below and estimate the cost to the nearest pound)

\section{Item bought}

1.

$£$

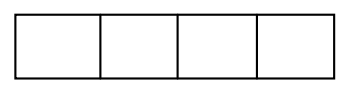

2.

$£$

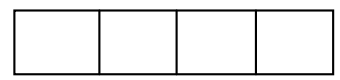

3.

$£$

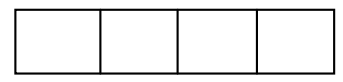

4.

$£$

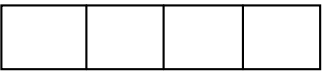

5.

$£$

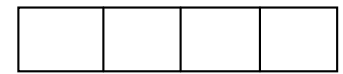

\section{Normal activities.}

11. Over the last $\mathbf{3}$ months has your back pain stopped you doing your normal activities?

Please cross any that apply and enter the total number of days your back pain stopped you getting on with your normal activities.

$\square \quad$ Employment

$\square \quad$ Education (i.e. College or University)

Housework

Childcare or care of a relative
Number of days

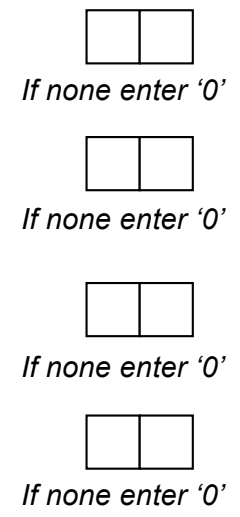




\section{Changes in work status in the last three months}

12. Have you had to take any days off sick from work in the last three months due to your low back pain?

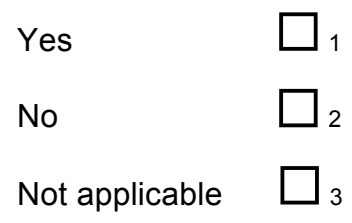

If Yes, how many days?

13. Have your hours of employment altered in the last three months because of changes in your back pain?

$\begin{array}{ll}\text { No, stayed the same } & \square_{1} \\ \text { Yes, increased } & \square_{2} \\ \text { Yes, decreased } & \square_{3} \\ \text { Not applicable } & \square_{4}\end{array}$

If Yes, by how many hours per week has your employment changed?

When did this change occur?

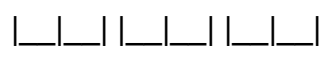

14. If you were off work when you joined the study, have you returned to work?

$\square$ I l am still off work

$\square$ I returned to work on

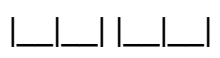




\section{Benefits and entitlement to free prescriptions}

15. Has your benefit status changed in the last three months?

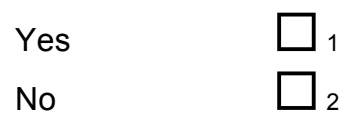

If yes, please cross all benefits that you are currently receiving.

$\begin{array}{llll}\text { Statutory Sick Pay } & \square_{1} & \text { Income support } & \square_{8} \\ \text { Incapacity Benefit } & \square_{2} & \text { Working tax credit } & \square_{9} \\ \text { Disability living allowance } & \square_{3} & \text { Child tax credit } & \square_{10} \\ \text { Severe disablement allowance } & \square_{4} & \text { Council tax benefit } & \square_{11} \\ \text { Disabled persons tax credit } & \square_{5} & \text { Housing benefit } & \square_{12} \\ \text { Carers allowance } & \square_{6} & \text { Attendance allowance } & \square_{13} \\ \text { Job seekers allowance } & \square & \text { Pension credit } & \square_{14} \\ & & \text { Other (please specify) } & \square 15 \\ & & & \end{array}$

THANK YOU FOR COMPLETING THIS QUESTIONNAIRE

If you have any comments, please write them overleaf. 
Your comments: 
In confidence

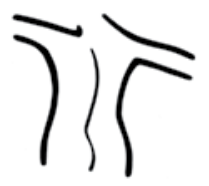

Potential participant study ID number

Back Skills Training (BeST) Trial

Trial Participant Six Month Follow Up Questionnaire

Back Skills Training (BeST) Trial

The University of Warwick

Centre for Primary Health Care

THE UNIVERSITY OF

WARWICK

MRC General Practice Research

Framework

A randomised study of treatments for back pain in primary care, funded by the NHS R\&D HTA programme (ISRCTN54717854) 


\section{PLEASE READ ALL THE INSTRUCTIONS BEFORE COMPLETING THE QUESTIONNAIRE}

Thank you for agreeing to take part in this study. The responses you give in this questionnaire will help us find out if the treatment you have had for your back since you joined the study has been helpful.

Please answer all the questions. Although it may seem that questions are asked more than once, it is still important that you answer every one.

Please follow the instructions for each section carefully.

For each section, if you are asked to put a cross in the box, please use a cross rather than a tick.

For example, in the following question if your answer to the question is yes, you should place a cross firmly in the box next to yes.

Do you drive a car?

Yes $\square$

No

If you are asked to circle a number, please use a circle rather than underlining a number.

For example, in the following question if you are asked 'how happy are you today?', where ' 1 ' is 'very unhappy' and ' 5 ' is 'very happy'. If you feel neither happy nor unhappy you may wish to answer ' 3 '. You do this by clearly circling the number 3.
1 2

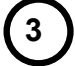
4

Please use a BLACK or BLUE pen. Please do not use a pencil.

All the responses you give will be kept strictly confidential. 
Firstly, please enter the date you are completing this questionnaire:

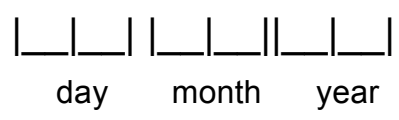

\section{Section 1}

By placing a cross in one box for each question below, please indicate which statement best describes your feelings towards the treatment or advice you have received for your lower back pain since you joined the study.

1. How satisfied are you with the treatment you received?

Very dissatisfied

$\square$

Somewhat dissatisfied

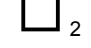

Neither satisfied nor dissatisfied

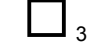

Somewhat satisfied

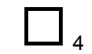

Very satisfied

2. How much benefit have you gained from the treatment or advice you have received for your lower back pain since you joined the study.

Substantial benefit<smiles>C1CCCC1</smiles>

Moderate benefit<smiles>C1CCC1</smiles>

No benefit<smiles>C1CCC1</smiles>

Moderate harm<smiles>C1CCC1</smiles>

Substantial harm 


\section{Section 2}

When your back hurts, you may find it difficult to do some of the things you normally do. This list contains some sentences that people have used to describe themselves when they have back pain. When you read them, you may find that some stand out because they describe you today.

As you read the list, think of yourself today. When you read a sentence that describes you today, place a cross in the box beside it. If the sentence does not describe you, then leave the box blank and go on to the next one. Remember, only place a cross if you are sure that it describes you today.

1. I stay at home most of the time because of my back.

2. I change positions frequently to try and get my back comfortable.

3. I walk more slowly than usual because of my back.

4. Because of my back, I am not doing any of the jobs that I usually do around the house.

5. Because of my back, I use a handrail to get upstairs

6. Because of my back, I lie down to rest more often.

7. Because of my back, I have to hold on to something to get out of an easy chair.

8. Because of my back, I try to get other people to do things for me.

9. I get dressed more slowly than usual because of my back.

10. I only stand up for short periods of time because of my back.

11. Because of my back, I try not to bend or kneel down.

12. I find it difficult to get out of a chair because of my back.

13. My back is painful almost all the time.

14. I find it difficult to turn over in bed because of my back.

15. My appetite is not very good because of my back pain.

16. I have trouble putting on my socks (or stockings) because of the pain in my back.

17. I only walk short distances because of my back pain.

18. I sleep less well because of my back.

19. Because of my back pain, I get dressed with help from someone else.

20. I sit down for most of the day because of my back.

21. I avoid heavy jobs around the house because of my back.

22. Because of my back pain, I am more irritable and bad tempered with people than usual.

23. Because of my back, I go upstairs more slowly than usual.

24. I stay in bed most of the time because of my back. 


\section{Section 3}

This section is about how much your back trouble has been interfering with your daily activities in recent weeks.

For the next six questions please circle the number which represents how your back pain has made you feel over the last $\underline{4 \text { weeks. }}$

For example:

$$
\begin{array}{lllllllllll}
0 & 1 & 2 & 3 & 4 & (5 & 6 & 7 & 8 & 9 & 10
\end{array}
$$

1. In the past $\mathbf{4}$ weeks, how much has your back pain interfered with your daily activities on a scale of $0-10$ where 0 is 'no interference' and 10 is 'unable to carry out any activities at all'?

$$
\begin{array}{lllllllllll}
0 & 1 & 2 & 3 & 4 & 5 & 6 & 7 & 8 & 9 & 10
\end{array}
$$

2. In the past $\mathbf{4}$ weeks, how much has your back pain changed your ability to take part in recreational, social and family activities on a scale of $0-10$ where $\underline{0 \text { is 'no change' and } \underline{10} \text { is }}$ 'extreme change'?

$\begin{array}{lllllllllll}0 & 1 & 2 & 3 & 4 & 5 & 6 & 7 & 8 & 9 & 10\end{array}$

3. In the past $\mathbf{4}$ weeks, how much has your back pain changed your ability to work (including

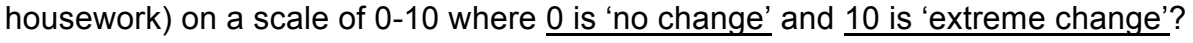

$\begin{array}{lllllllllll}0 & 1 & 2 & 3 & 4 & 5 & 6 & 7 & 8 & 9 & 10\end{array}$

4. In the past $\mathbf{4}$ weeks, how bad has your worst back pain been on a scale of $0-10$ where $\underline{0 \text { is }}$ 'no pain' and 10 is 'as bad as a pain could be'?

$\begin{array}{lllllllllll}0 & 1 & 2 & 3 & 4 & 5 & 6 & 7 & 8 & 9 & 10\end{array}$

5. In the past $\mathbf{4}$ weeks, on average how bad has your back pain been on a scale of $0-10$

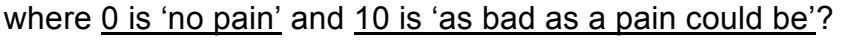

$\begin{array}{lllllllllll}0 & 1 & 2 & 3 & 4 & 5 & 6 & 7 & 8 & 9 & 10\end{array}$

6. How would you rate your back pain today on a scale of $0-10$ where $\underline{0 \text { is 'no pain' and } 10 \text { is }}$ 'as bad as a pain could be'?

$\begin{array}{lllllllllll}0 & 1 & 2 & 3 & 4 & 5 & 6 & 7 & 8 & 9 & 10\end{array}$




\section{Section 4}

Please rate how confident you are that you can do the following things at present, despite the pain. To answer, circle one of the numbers on the scale under each item, where $0=$ 'not at all confident' and $6=$ 'completely confident'.

For example

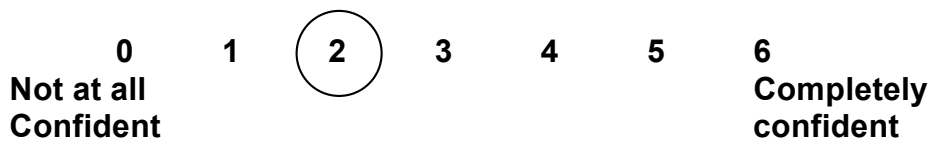

Remember, this questionnaire is not asking whether or not you have been doing these things, but rather how confident you are that you can do them at present, despite the pain.

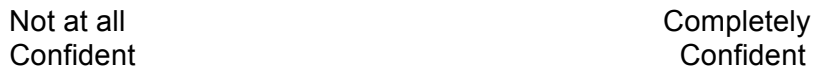

1. I can enjoy things, despite the pain

$\begin{array}{lllllll}0 & 1 & 2 & 3 & 4 & 5 & 6 \\ 0 & 1 & 2 & 3 & 4 & 5 & 6\end{array}$

2. I can do most of the household chores (e.g. tidying-up, washing dishes, etc.), despite the pain

3. I can socialise with my friends or family members as often as I used to do, despite the pain

4. I can cope with my pain in most situations

5. I can do some form of work, despite the pain. ('work' includes housework, paid and unpaid work).

6. I can still do many of the things I enjoy doing, such as hobbies or leisure activity, despite the pain

7. I can cope with my pain without medication

$\begin{array}{lllllll}0 & 1 & 2 & 3 & 4 & 5 & 6 \\ 0 & 1 & 2 & 3 & 4 & 5 & 6\end{array}$

8. I can still accomplish most of my goals in life, despite the pain

9. I can live a normal lifestyle, despite the pain

10. I can gradually become more active, despite the pain 


\section{$\underline{\text { Section } 5}$}

These are some things people have told us about their back pain. For each statement please circle a number from 0 to 6 to say how much physical activity such as bending, lifting, driving affect your pain.

Please circle one number for each line

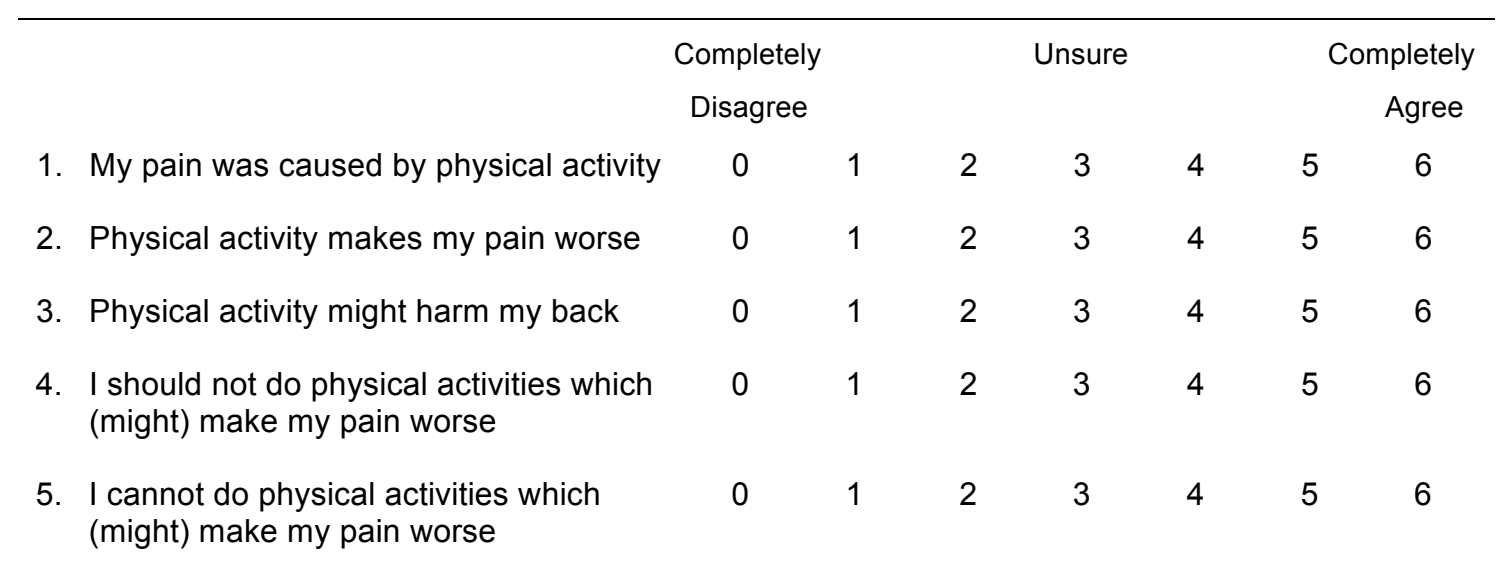

\section{Section 6}

This section is to determine how much your low back pain has troubled you lately.

1. During the past 4 weeks, about how many days did low back pain keep you from going to work or school/college/university? days

2. During the past 4 weeks, about how many days did you have to cut down on the things you usually do for more than half the day because of back pain? days

3. How would you describe your back pain compared to when you started the study? Very much improved (or completely recovered)

Much improved

Minimally (or slightly) improved

No change

Minimally worse<smiles>[CH]1CCC1</smiles>

Much worse

Very much worse 


\section{Section 7}

This section asks for your views about your health. This information will help keep track of how you feel and how well you are able to do your usual activities.

For each of the following questions, please place a cross in the one box that best describes your answer.

1. In general, would you say your health is:
Excellent
Very good
Good
Fair
Poor
$\square 1$
$\square_{2}$
$\square 3$
$\square_{4}$
$\square 5$

2. The following questions are about activities you might do during a typical day.

Does your health now limit you in these activities? If so, how much?

$\begin{array}{ccc}\begin{array}{c}\text { Yes, } \\ \text { limited } \\ \text { a lot }\end{array} & \begin{array}{c}\text { Yes, } \\ \text { limited } \\ \text { a little }\end{array} & \begin{array}{c}\text { No, not } \\ \text { limited } \\ \text { at all }\end{array} \\ \square_{1} & \square_{2} & \square_{3} \\ \square_{1} & \square_{2} & \square_{3}\end{array}$

a) Moderate activities, such as moving a table, pushing a
vacuum cleaner, bowling or playing golf
b) Climbing several flights of stairs
3. During the past 4 weeks, how much of the time have you had any of the following problems
with your work or other regular daily activities as a result of your physical health?

a) Moderate activities, such as moving a table, pushing a
vacuum cleaner, bowling or playing golf
b) Climbing several flights of stairs
3. During the past 4 weeks, how much of the time have you had any of the following p
with your work or other regular daily activities as a result of your physical health?

$\begin{array}{ccccc}\begin{array}{c}\text { All of } \\ \text { the time }\end{array} & \begin{array}{c}\text { Most of } \\ \text { the time }\end{array} & \begin{array}{l}\text { Some of } \\ \text { the time }\end{array} & \begin{array}{c}\text { A little of } \\ \text { the time }\end{array} & \begin{array}{c}\text { None of } \\ \text { the time }\end{array}\end{array}$

a) Accomplished less than you would like

b) Were limited in the kind of work or other activities

$\begin{array}{lllll}\square_{1} & \square_{2} & \square_{3} & \square_{4} & \square 5 \\ \square_{1} & \square_{2} & \square_{3} & \square_{4} & \square_{5}\end{array}$


4. During the past $\mathbf{4}$ weeks, how much of the time have you had any of the following problems with your work or other regular activities as a result of any emotional problems (such as feeling depressed or anxious)?

\begin{tabular}{llcccc|}
\hline & $\begin{array}{c}\text { All of } \\
\text { the time }\end{array}$ & $\begin{array}{c}\text { Most of } \\
\text { the time }\end{array}$ & $\begin{array}{c}\text { Some of } \\
\text { the time }\end{array}$ & $\begin{array}{c}\text { A little of } \\
\text { the time }\end{array}$ & $\begin{array}{c}\text { None of } \\
\text { the time }\end{array}$ \\
a) $\begin{array}{l}\text { Accomplished less than } \\
\text { you would like }\end{array}$ & $\square_{1}$ & $\square_{2}$ & $\square_{3}$ & \\
b) $\begin{array}{l}\text { Were limited in the kind of } \\
\text { work or other activities }\end{array}$ & $\square_{1}$ & $\square_{2}$ & $\square_{3}$ & \\
\hline
\end{tabular}

5. During the past $\mathbf{4}$ weeks, how much did pain interfere with your normal work (including both work outside the home and housework)?

$\begin{array}{ccccc}\text { Not at all } & \text { A little bit } & \text { Moderately } & \text { Quite a bit } & \text { Extremely } \\ \square_{1} & \square_{2} & \square_{3} & \square_{4} & \square_{5}\end{array}$

6. These questions are about how you feel and how things have been with you during the past 4 weeks. For each question, please give the one answer that comes closest to the way you have been feeling. How much of the time during the past 4 weeks:

a) Have you felt calm and peaceful?

$\begin{array}{ccccc}\begin{array}{c}\text { All of the } \\ \text { time }\end{array} & \begin{array}{c}\text { Most of } \\ \text { the time }\end{array} & \begin{array}{c}\text { Some of } \\ \text { the time }\end{array} & \begin{array}{c}\text { A little of } \\ \text { the time }\end{array} & \begin{array}{c}\text { None of } \\ \text { the time }\end{array}\end{array}$

b) Did you have a lot of energy?
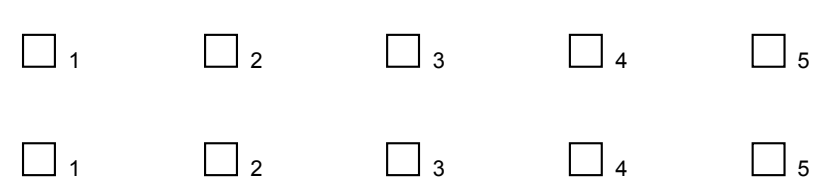

$\square_{2}$

3

c) Have you felt downhearted and low?

$\square_{2}$
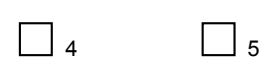

$\square_{3}$

4 5 5

7. During the past 4 weeks, how much of the time has your physical health OR emotional problems interfered with your social activities (like visiting with friends, relatives, etc.)?

$\begin{array}{ccccc}\begin{array}{c}\text { All of } \\ \text { the time }\end{array} & \begin{array}{c}\text { Most of } \\ \text { the time }\end{array} & \begin{array}{c}\text { Some of } \\ \text { the time }\end{array} & \begin{array}{c}\text { A little of } \\ \text { the time }\end{array} & \begin{array}{c}\text { None of } \\ \text { the time }\end{array} \\ \square_{1} & \square_{2} & \square_{3} & \square_{4} & \square_{5}\end{array}$




\section{Section 8}

The following questions are to ask about your general health state at the moment. By placing a cross (' $X$ ') in one box in each group below, please indicate which statement best describes your own health state today.

Do not cross more than one box per question.

1. Mobility:

I have no problems in walking about

I have some problems in walking about

I am confined to bed

2. Self-Care:

I have no problems with self-care

I have some problems washing or dressing myself

I am unable to wash or dress myself

3. Usual Activities (e.g. work, study, housework, family or leisure activities):

I have no problems with performing my usual activities

I have some problems with performing my usual activities

I am unable to perform my usual activities

4. Pain / Discomfort:

I have no pain or discomfort

I have moderate pain or discomfort

I have extreme pain or discomfort

\section{Anxiety / Depression:}

I am not anxious or depressed

I am moderately anxious or depressed

I am extremely anxious or depressed 


\section{Your own health state today}

To help people say how good or bad a health state is, we have drawn a scale (rather like a thermometer) on which the best state you can imagine is marked by 100 and the worst state you can imagine is marked by 0 .

We would like you to indicate on this scale how good or bad is your own health today, in your opinion.

Please do this by drawing a line from the box below, to whichever point on the scale indicates how good or bad your current health state is today.

Your own health state TODAY
Best

imaginable
hearth state

100

F

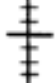

$\neq$

$\neq$

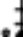

龺

㱏

要。

美

龺。

量

$\bar{F}$

主

年

Worst

naginable

health state 


\section{Section 9}

This section is about health care you have received for your back pain. There are separate parts for NHS treatment, private treatment, products/equipment, normal activities and any benefits/entitlements. Please read each question carefully. For each question, if you have had no treatments or visits, please enter ' 0 '.

\section{NHS Treatment}

1. In the last $\mathbf{3}$ months, how often have you attended the following NHS services for lower back pain? (Please do not include any sessions or treatments that you attended as part of the study).

Number of times

Your GP or another GP

Practice nurse

Physiotherapist

Doctor/nurse in an accident and emergency department

(Casualty)

Hospital specialist (consultant or one of his/her team)

Psychologist

Counsellor

Other (please specify)

Other (please specify)

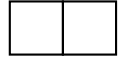

if none enter ' 0 '

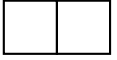

if none enter ' 0 '

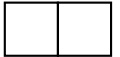

if none enter ' 0 '

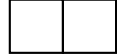

if none enter ' 0 '

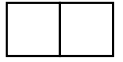

if none enter ' 0 '

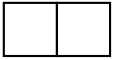

if none enter ' 0 '

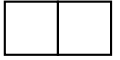

if none enter ' 0 '

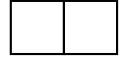

if none enter ' 0 '

if none enter ' 0 '

2. In the last $\mathbf{3}$ months have you been admitted to an NHS hospital because of back pain?

$$
\begin{array}{ll}
\text { Yes } & \square 1 \\
\text { No } & \square_{2}
\end{array}
$$

If Yes, in total, how many days were you in hospital? 
3. In the last $\mathbf{3}$ months have you had any of the following tests in an NHS hospital in relation to lower back pain?

Number of times

X-ray

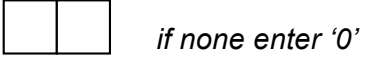

CT Scan

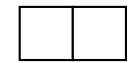

if none enter ' 0 '

MRI Scan

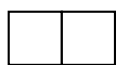

if none enter ' 0 '

Blood tests (count all blood tests done on one day as one test)

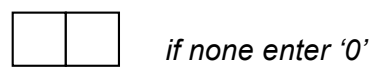

Other (please specify)

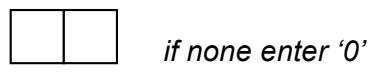

4. In the last $\mathbf{3}$ months has your doctor prescribed any of the following medications for your back?

Pain killers

Anti-inflammatory drugs (for example ibuprofen, naproxen)

Gels/Creams (for example ibuleve or movelat)

Sleeping pills

Anti-depressants

Other (Please specify)
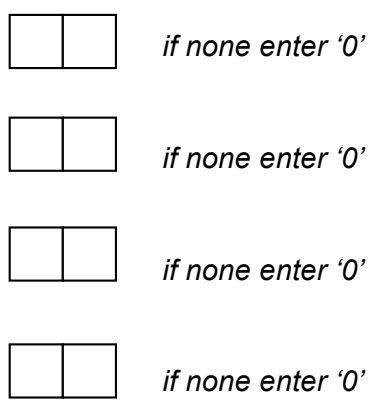

if none enter ' 0 ' 


\section{Private Treatment}

5. For the last $\mathbf{3}$ months please detail total treatment costs you paid for yourself; or paid for by private insurance; please do not include any treatments paid for by the NHS. Please round the amounts to the nearest pound.

$\begin{array}{lll}\text { Number } & \text { Medical } & \text { Personal } \\ \text { Of times } & \text { Insurance } & \text { Contribution } \\ & \text { Contribution } & \end{array}$

Private physiotherapist
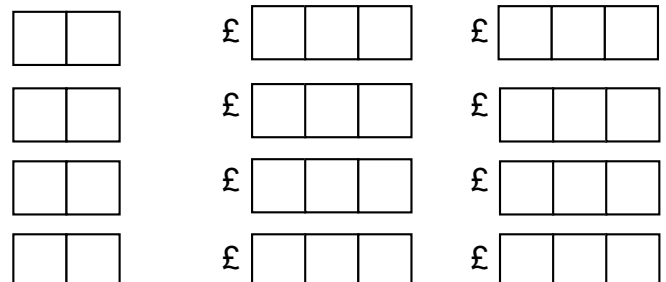

Private hospital specialist (consultant)
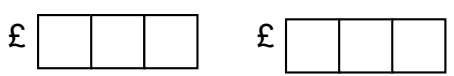

Private osteopath

Private chiropractor
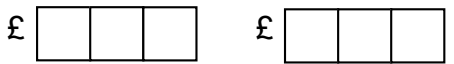

$£$

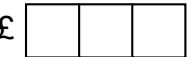

Private psychologist

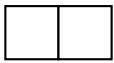

$£$

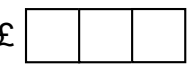

Private counsellor

Private massage therapist

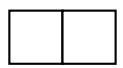

$£$

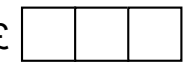

Private massage therapist
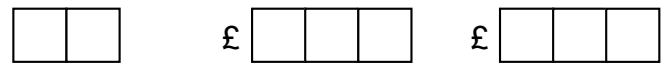

Private aroma therapist
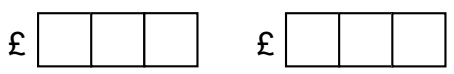

Private acupuncturist
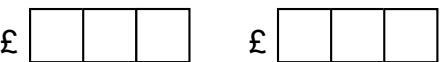

Other (please specify)
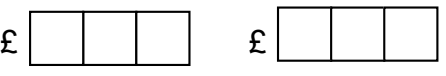

Other (please specify)
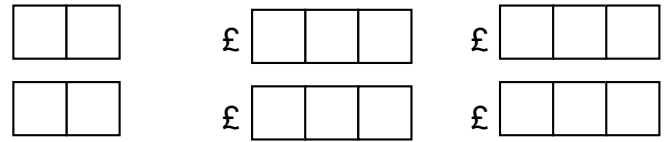

6. In the last $\mathbf{3}$ months, have you been admitted to a private hospital because of lower back pain?

$\begin{array}{ll}\text { Yes } & \square \\ \text { No } & \square\end{array}$

If Yes, in total, how many days were you in hospital?

If Yes, what were the total costs paid by your medical insurance? $£$

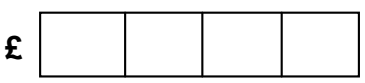

Please give the total costs to the nearest pound*.

If Yes, what were the total costs paid by you?

$\boldsymbol{f}$

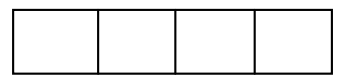

Please give the total costs to the nearest pound.*

*If you do not know the actual cost please give us your best estimate of the costs. 
7. In the last $\mathbf{3}$ months, have you had any of the following tests in a private hospital in relation to lower back pain?

$$
\text { Number of times }
$$

X-ray

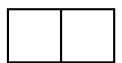

if none enter ' 0 '

CT Scan

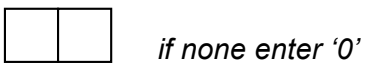

MRI Scan

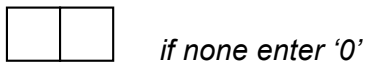

Blood tests (please count all blood tests done on one day as one test)

if none enter ' 0 '

Other (please specify)

if none enter ' 0 '

8. In the last 3 months have you bought (other than by a prescription) any of the following treatments for your back pain? Please estimate the total cost to the nearest pound.

Number

Total cost

of times

to you

Pain killers
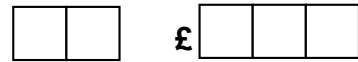

Anti-inflammatory drugs (for example ibuprofen/nurofen)
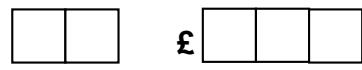

Gels/Creams (for example ibuleve or movelat)
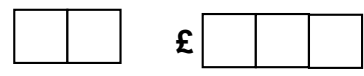

Other (Please specify) 


\section{Products / equipment.}

9. In the last three months, have you bought items such as braces or aids, a new bed or mattress, a chair, a massage machine, or any other products or equipment because of your back pain? (please list the item below and estimate the cost to the nearest pound)

\section{Item bought}

1.

.

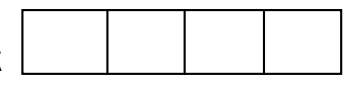

2.

$£$

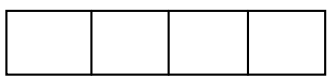

3.

$£$

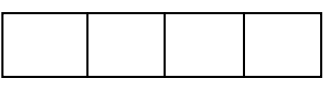

4.

$£$

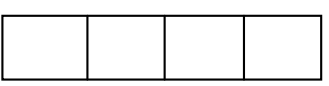

5.

$£$

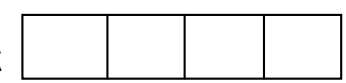

\section{Normal activities.}

10. Over the last $\mathbf{3}$ months has your back pain stopped you doing your normal activities?

Please cross any that apply and enter the total number of days your back pain stopped you getting on with your normal activities.

Employment

Education (i.e. college or University)

Housework

Childcare or care of a relative
Number of days

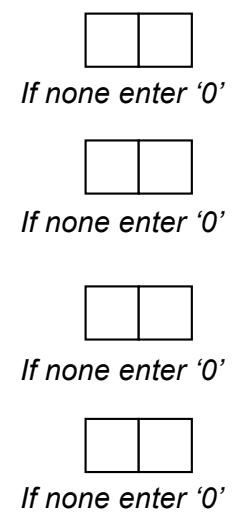




\section{Changes in work status in the last three months}

11. Have you had to take any days off sick from work in the last three months due to your low back pain?

$\begin{array}{ll}\text { Yes } & \square \text { No } \\ \text { Not applicable } & \square{ }_{3}\end{array}$

If Yes, how many days?

12. Have you had to change your occupation in the last three months due to your back pain?

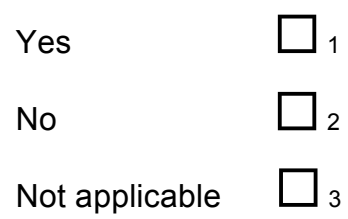

If Yes, what is your new job?

When did this change occur? I_L__ I____l I__

13. Have your hours of employment changed in the last three months because of back pain getting better or worse?

$\begin{array}{ll}\text { Yes } & \square_{1} \\ \text { No } & \square_{2} \\ \text { Not applicable } & \square{ }_{3}\end{array}$

If Yes, by how many hours per week has your employment changed?

When did this change occur?

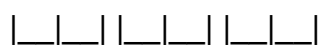

14. If you were off work when you joined the study, have you returned to work?

$\square$ I l am still off work

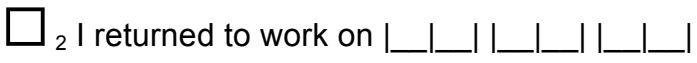




\section{Benefits and entitlement to free prescriptions}

15. Do you qualify for free prescriptions?

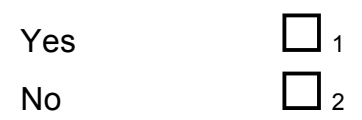

16. Has your benefit status changed in the last three months?

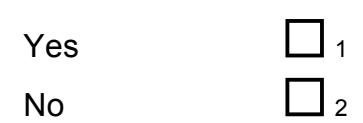

If yes, please cross all benefits that you are currently receiving.

$\begin{array}{lll}\text { Statutory Sick Pay } & \square_{1} & \text { Child Tax Credit } \\ \text { Incapacity Benefit } & \square_{2} \quad \text { Council Tax Benefit } \\ \text { Disability living allowance } & \square_{3} \quad \text { Housing Benefit } \\ \text { Disabled persons tax credit } & \square_{4} \quad \text { Attendance allowance } \\ \text { Invalid Care Allowance } & \square_{5} \text { (includes if paid to someone who looks after you) } \\ \text { Other } & \square_{6} \text { please specify }\end{array}$

Working Tax Credit $\quad \square 7$

THANK YOU FOR COMPLETING THIS QUESTIONNAIRE

If you have any comments, please write them overleaf. 


\section{Your comments:}


In confidence

Potential participant study ID number

Back Skills Training (BeST) Trial

Trial Participant Twelve Month Follow Up Questionnaire

Back Skills Training (BeST) Trial

The University of Warwick

Centre for Primary Health Care
THE UNIVERSITY OF WARWICK

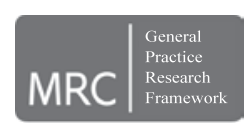

MRC General Practice Research Framework

A randomised study of treatments for back pain in primary care, funded by the NHS R\&D HTA programme (ISRCTN54717854) 


\section{PLEASE READ ALL THE INSTRUCTIONS BEFORE COMPLETING THE QUESTIONNAIRE}

Thank you for agreeing to take part in this study. The responses you give in this questionnaire will help us find out if the treatment you have had for your back since you joined the study has been helpful.

Please answer all the questions. Although it may seem that questions are asked more than once, it is still important that you answer every one.

Please follow the instructions for each section carefully.

For each section, if you are asked to put a cross in the box, please use a cross rather than a tick.

For example, in the following question if your answer to the question is yes, you should place a cross firmly in the box next to yes.

Do you drive a car? Yes $\bigotimes$

No

If you are asked to circle a number, please use a circle rather than underlining a number.

For example, in the following question if you are asked 'how happy are you today?', where ' 1 ' is 'very unhappy' and ' 5 ' is 'very happy'. If you feel neither happy nor unhappy you may wish to answer ' 3 '. You do this by clearly circling the number 3.

1 2

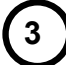

Please use a BLACK or BLUE pen. Please do not use a pencil.

All the responses you give will be kept strictly confidential. 
Firstly, please enter the date you are completing this questionnaire:

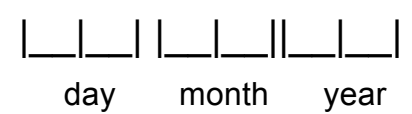

\section{Section 1}

By placing a cross in one box for each question below, please indicate which statement best describes your feelings towards the treatment or advice you have received for your lower back pain since you joined the study.

1. How satisfied are you with the treatment you received?

$\begin{array}{ll}\text { Very dissatisfied } & \square \\ \text { Somewhat dissatisfied } & \square_{2} \\ \text { Neither satisfied nor dissatisfied } & \square_{3} \\ \text { Somewhat satisfied } & \square_{4} \\ \text { Very satisfied } & \square_{5}\end{array}$

2. How much benefit have you gained from the treatment or advice you have received for your lower back pain since you joined the study.

$\begin{array}{ll}\text { Substantial benefit } & \square_{1} \\ \text { Moderate benefit } & \square_{2} \\ \text { No benefit } & \square_{3} \\ \text { Moderate harm } & \square_{5}\end{array}$




\section{Section 2}

When your back hurts, you may find it difficult to do some of the things you normally do. This list contains some sentences that people have used to describe themselves when they have back pain. When you read them, you may find that some stand out because they describe you today.

As you read the list, think of yourself today. When you read a sentence that describes you today, place a cross in the box beside it. If the sentence does not describe you, then leave the box blank and go on to the next one. Remember, only place a cross if you are sure that it describes you today.

1. I stay at home most of the time because of my back.

2. I change positions frequently to try and get my back comfortable.

3. I walk more slowly than usual because of my back.

4. Because of my back, I am not doing any of the jobs that I usually do around the house.

5. Because of my back, I use a handrail to get upstairs

6. Because of my back, I lie down to rest more often.

7. Because of my back, I have to hold on to something to get out of an easy chair.

8. Because of my back, I try to get other people to do things for me.

9. I get dressed more slowly than usual because of my back.

10. I only stand up for short periods of time because of my back.

11. Because of my back, I try not to bend or kneel down.

12. I find it difficult to get out of a chair because of my back.

13. My back is painful almost all the time.

14. I find it difficult to turn over in bed because of my back.

15. My appetite is not very good because of my back pain.

16. I have trouble putting on my socks (or stockings) because of the pain in my back.

17. I only walk short distances because of my back pain.

18. I sleep less well because of my back.

19. Because of my back pain, I get dressed with help from someone else.

20. I sit down for most of the day because of my back.

21. I avoid heavy jobs around the house because of my back.

22. Because of my back pain, I am more irritable and bad tempered with people than usual.

23. Because of my back, I go upstairs more slowly than usual.

24. I stay in bed most of the time because of my back. 


\section{Section 3}

This section is about how much your back trouble has been interfering with your daily activities in recent weeks.

For the next six questions please circle the number which represents how your back pain has made you feel over the last $\underline{4 \text { weeks. }}$

For example:

$$
\begin{array}{lllllllllll}
0 & 1 & 2 & 3 & 4 & (5) & 6 & 7 & 8 & 9 & 10
\end{array}
$$

1. In the past $\mathbf{4}$ weeks, how much has your back pain interfered with your daily activities on a scale of $0-10$ where $\underline{0}$ is 'no interference' and 10 is 'unable to carry out any activities at all'?

$$
\begin{array}{lllllllllll}
0 & 1 & 2 & 3 & 4 & 5 & 6 & 7 & 8 & 9 & 10
\end{array}
$$

2. In the past $\mathbf{4}$ weeks, how much has your back pain changed your ability to take part in recreational, social and family activities on a scale of $0-10$ where $\underline{0 \text { is 'no change' and } \underline{10} \text { is }}$ 'extreme change'?

$\begin{array}{lllllllllll}0 & 1 & 2 & 3 & 4 & 5 & 6 & 7 & 8 & 9 & 10\end{array}$

3. In the past 4 weeks, how much has your back pain changed your ability to work (including housework) on a scale of $0-10$ where 0 is 'no change' and 10 is 'extreme change'?

$\begin{array}{lllllllllll}0 & 1 & 2 & 3 & 4 & 5 & 6 & 7 & 8 & 9 & 10\end{array}$

4. In the past $\mathbf{4}$ weeks, how bad has your worst back pain been on a scale of $0-10$ where $\underline{0 \text { is }}$ 'no pain' and 10 is 'as bad as a pain could be'?

$\begin{array}{lllllllllll}0 & 1 & 2 & 3 & 4 & 5 & 6 & 7 & 8 & 9 & 10\end{array}$

5. In the past $\mathbf{4}$ weeks, on average how bad has your back pain been on a scale of 0-10

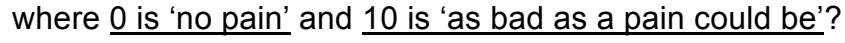

$\begin{array}{lllllllllll}0 & 1 & 2 & 3 & 4 & 5 & 6 & 7 & 8 & 9 & 10\end{array}$

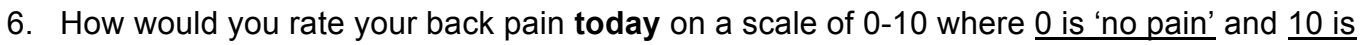
'as bad as a pain could be'?

$\begin{array}{lllllllllll}0 & 1 & 2 & 3 & 4 & 5 & 6 & 7 & 8 & 9 & 10\end{array}$




\section{Section 4}

Please rate how confident you are that you can do the following things at present, despite the pain. To answer, circle one of the numbers on the scale under each item, where $0=$ 'not at all confident' and $6=$ 'completely confident'.

For example

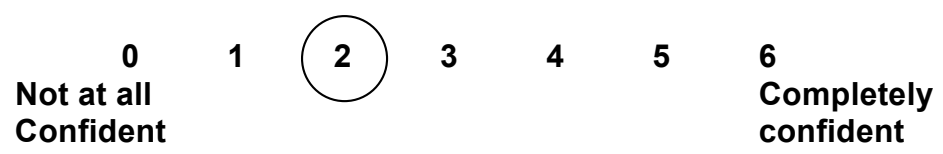

Remember, this questionnaire is not asking whether or not you have been doing these things, but rather how confident you are that you can do them at present, despite the pain.

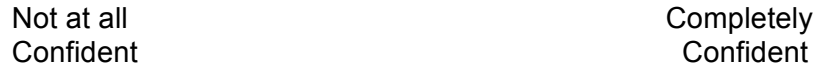

1. I can enjoy things, despite the pain

$\begin{array}{lllllll}0 & 1 & 2 & 3 & 4 & 5 & 6 \\ 0 & 1 & 2 & 3 & 4 & 5 & 6\end{array}$

I can do most of the household chores (e.g.
tidying-up, washing dishes, etc.), despite the pain

3. I can socialise with my friends or family members as often as I used to do, despite the pain

4. I can cope with my pain in most situations

$0 \quad 1$

5. I can do some form of work, despite the pain.

0
work).

6. I can still do many of the things I enjoy doing, such as hobbies or leisure activity, despite the pain

7. I can cope with my pain without medication

$\begin{array}{lllllll}0 & 1 & 2 & 3 & 4 & 5 & 6 \\ 0 & 1 & 2 & 3 & 4 & 5 & 6\end{array}$

8. I can still accomplish most of my goals in life, $\quad \begin{array}{lllllll}0 & 1 & 2 & 3 & 4 & 5 & 6\end{array}$ despite the pain

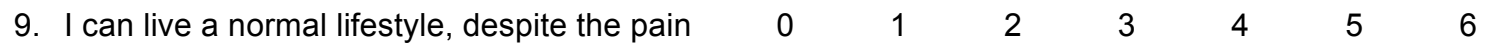

10. I can gradually become more active, despite $\quad 0 \quad 1 \quad 3 \quad 2 \quad 3 \quad 4 \quad 5 \quad 6$ the pain 


\section{Section 5}

These are some things people have told us about their back pain. For each statement please circle a number from 0 to 6 to say how much physical activity such as bending, lifting, driving affect your pain.

Please circle one number for each line

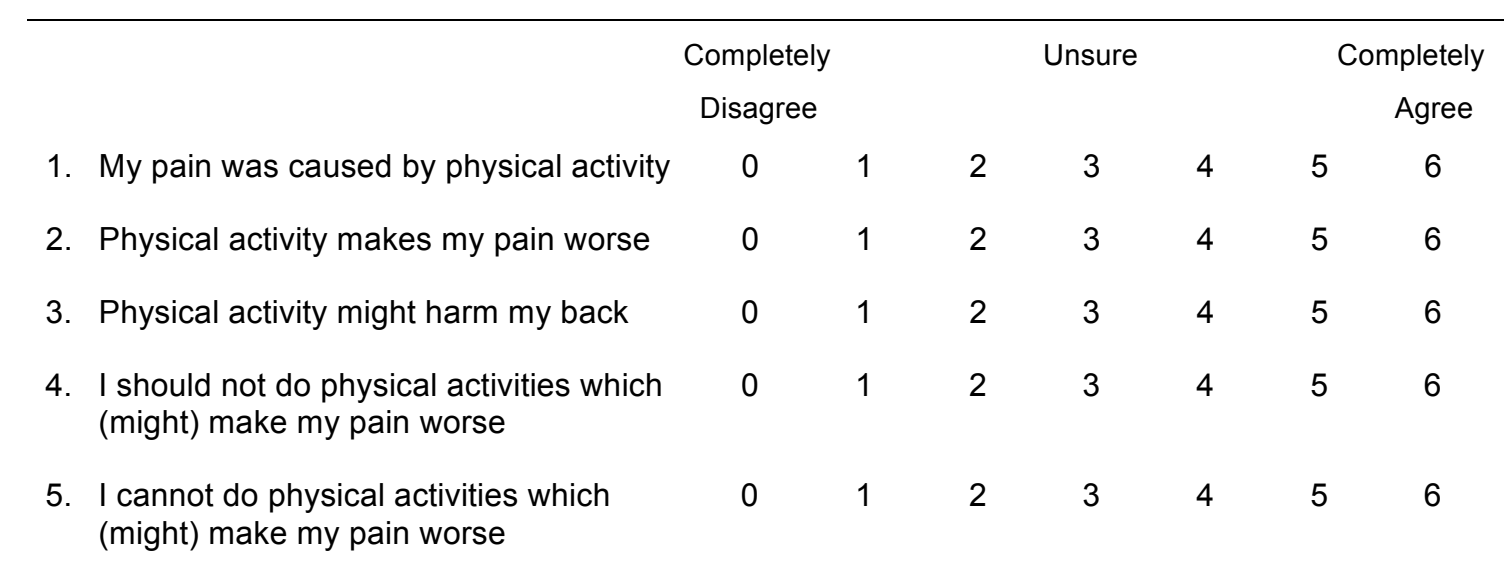

\section{Section 6}

This section is to determine how much your low back pain has troubled you lately.

1. During the past 4 weeks, about how many days did low back pain keep you from going to work or school/college/university? days

2. During the past 4 weeks, about how many days did you have to cut down on the things you usually do for more than half the day because of back pain? days

3. How would you describe your back pain compared to when you started the study? Very much improved (or completely recovered)

Much improved

Minimally (or slightly) improved<smiles>C1CCCC1</smiles>

No change<smiles>C1CCCC1</smiles>

Minimally worse<smiles>C1CCC1</smiles>

Much worse

Very much worse 


\section{Section 7}

This section asks for your views about your health. This information will help keep track of how you feel and how well you are able to do your usual activities.

For each of the following questions, please place a cross in the one box that best describes your answer.

1. In general, would you say your health is:

$\begin{array}{ccccc}\text { Excellent } & \text { Very good } & \text { Good } & \text { Fair } & \text { Poor } \\ \square_{1} & \square_{2} & \square_{3} & \square_{4} & \square_{5}\end{array}$

2. The following questions are about activities you might do during a typical day. Does your health now limit you in these activities? If so, how much?

$\begin{array}{ccc}\begin{array}{c}\text { Yes, } \\ \text { limited } \\ \text { a lot }\end{array} & \begin{array}{c}\text { Yes, } \\ \text { limited } \\ \text { a little }\end{array} & \begin{array}{c}\text { No, not } \\ \text { limited } \\ \text { at all }\end{array} \\ \square_{1} & \square_{2} & \square_{3} \\ \square_{1} & \square_{2} & \square_{3}\end{array}$

a) Moderate activities, such as moving a table, pushing a
vacuum cleaner, bowling or playing golf
b) Climbing several flights of stairs

a) Moderate activities, such as moving a table, pushing a
vacuum cleaner, bowling or playing golf
b) Climbing several flights of stairs
3. During the past 4 weeks, how much of the time have you had any of the following prob
with your work or other regular daily activities as a result of your physical health?

$\begin{array}{ccccc}\begin{array}{c}\text { All of } \\ \text { the time }\end{array} & \begin{array}{c}\text { Most of } \\ \text { the time }\end{array} & \begin{array}{c}\text { Some of } \\ \text { the time }\end{array} & \begin{array}{c}\text { A little of } \\ \text { the time }\end{array} & \begin{array}{c}\text { None of } \\ \text { the time }\end{array} \\ \square_{1} & \square_{2} & \square_{3} & \square_{4} & \square_{5} \\ \square_{1} & \square_{2} & \square_{3} & \square_{4} & \square_{5}\end{array}$


4. During the past $\mathbf{4}$ weeks, how much of the time have you had any of the following problems with your work or other regular activities as a result of any emotional problems (such as feeling depressed or anxious)?

\begin{tabular}{|lccccc|}
\hline & $\begin{array}{c}\text { All of } \\
\text { the time }\end{array}$ & $\begin{array}{c}\text { Most of } \\
\text { the time }\end{array}$ & $\begin{array}{c}\text { Some of } \\
\text { the time }\end{array}$ & $\begin{array}{c}\text { A little of } \\
\text { the time }\end{array}$ & $\begin{array}{c}\text { None of } \\
\text { the time }\end{array}$ \\
a) $\begin{array}{l}\text { Accomplished less than } \\
\text { you would like }\end{array}$ & $\square_{1}$ & $\square_{2}$ & $\square_{3}$ & \\
b) $\begin{array}{l}\text { Were limited in the kind of } \\
\text { work or other activities }\end{array}$ & $\square_{1}$ & $\square_{2}$ & $\square_{3}$ & $\square_{4}$
\end{tabular}

5. During the past 4 weeks, how much did pain interfere with your normal work (including both work outside the home and housework)?

$\begin{array}{ccccc}\text { Not at all } & \text { A little bit } & \text { Moderately } & \text { Quite a bit } & \text { Extremely } \\ \square_{1} & \square_{2} & \square_{3} & \square_{4} & \square_{5}\end{array}$

6. These questions are about how you feel and how things have been with you during the past 4 weeks. For each question, please give the one answer that comes closest to the way you have been feeling. How much of the time during the past 4 weeks:

$\begin{array}{ccccc}\text { All of the } & \begin{array}{c}\text { Most of } \\ \text { time }\end{array} \text { the time } & \begin{array}{c}\text { Some of } \\ \text { the time }\end{array} & \begin{array}{c}\text { A little of } \\ \text { the time }\end{array} & \begin{array}{c}\text { None of } \\ \text { the time }\end{array}\end{array}$

a) Have you felt calm and peaceful?
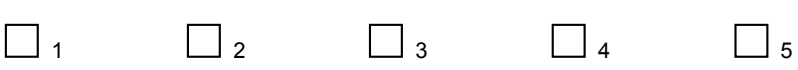

b) Did you have a lot of energy?

c) Have you felt downhearted and low?
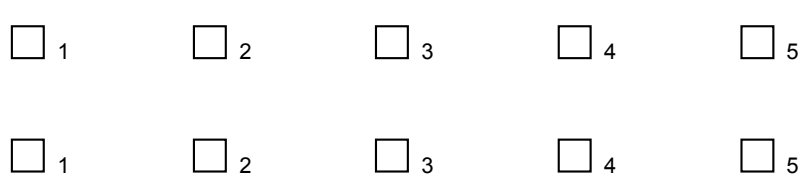
5

7. During the past $\mathbf{4}$ weeks, how much of the time has your physical health OR emotional problems interfered with your social activities (like visiting with friends, relatives, etc.)?

$\begin{array}{ccccc}\begin{array}{c}\text { All of } \\ \text { the time }\end{array} & \begin{array}{c}\text { Most of } \\ \text { the time }\end{array} & \begin{array}{c}\text { Some of } \\ \text { the time }\end{array} & \begin{array}{c}\text { A little of } \\ \text { the time }\end{array} & \begin{array}{c}\text { None of } \\ \text { the time }\end{array} \\ \square_{1} & \square_{2} & \square_{3} & \square_{4} & \square_{5}\end{array}$




\section{Section 8}

The following questions are to ask about your general health state at the moment. By placing a cross (' $X$ ') in one box in each group below, please indicate which statement best describes your own health state today.

\section{Do not cross more than one box per question.}

\section{Mobility:}

I have no problems in walking about

I have some problems in walking about

I am confined to bed

\section{Self-Care:}

I have no problems with self-care

I have some problems washing or dressing myself

I am unable to wash or dress myself

3. Usual Activities (e.g. work, study, housework, family or leisure activities):

I have no problems with performing my usual activities

I have some problems with performing my usual activities

I am unable to perform my usual activities

\section{Pain / Discomfort:}

I have no pain or discomfort

I have moderate pain or discomfort

I have extreme pain or discomfort

\section{Anxiety / Depression:}

I am not anxious or depressed

I am moderately anxious or depressed

I am extremely anxious or depressed 


\section{Your own health state today}

To help people say how good or

bad a health state is, we have

drawn a scale (rather like a thermometer) on which the best state you can imagine is marked by 100 and the worst state you can imagine is marked by 0 .

We would like you to indicate on this scale how good or bad is your own health today, in your opinion.

Please do this by drawing a line from the box below, to whichever point on the scale indicates how good or bad your current health state is today.

Your own health state TODAY

100

F

至

900

奉

声

姜 


\section{Section 9}

This section is about health care you have received for your back pain. There are separate parts for NHS treatment, private treatment, products/equipment, normal activities and any benefits/entitlements. Please read each question carefully. For each question, if you have had no treatments or visits, please enter ' 0 '.

\section{NHS Treatment}

1. In the last 6 months, how often have you attended the following NHS services for lower back pain? (Please do not include any sessions or treatments that you attended as part of the study).

Number of times

Your GP or another GP

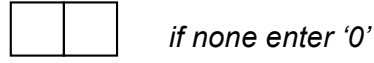

Practice nurse

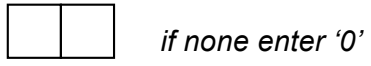

Physiotherapist

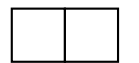

if none enter ' 0 '

Doctor/nurse in an accident and emergency department

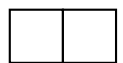

if none enter ' 0 '

(Casualty)

Hospital specialist (consultant or one of his/her team)

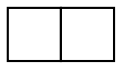

if none enter ' 0 '

Psychologist

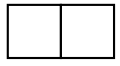

if none enter ' 0 '

Counsellor

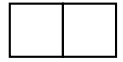

if none enter ' 0 '

Other (please specify)

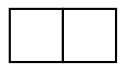

if none enter ' 0 '

Other (please specify)

if none enter ' 0 '

2. In the last $\mathbf{6}$ months have you been admitted to an NHS hospital because of back pain?

$$
\begin{array}{ll}
\text { Yes } & \square 1 \\
\text { No } & \square_{2}
\end{array}
$$

If Yes, in total, how many days were you in hospital? 
3. In the last 6 months have you had any of the following tests in an NHS hospital in relation to lower back pain?

\section{Number of times}

X-ray

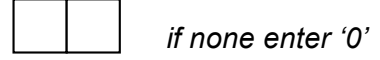

CT Scan

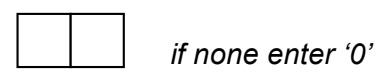

MRI Scan

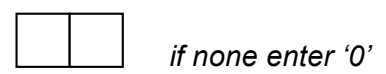

Blood tests (count all blood tests done on one day as one test)

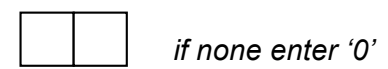

Other (please specify)

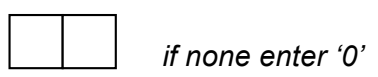

4. In the last 6 months has your doctor prescribed any of the following medications for your back?

Pain killers

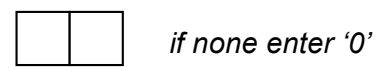

Anti-inflammatory drugs (for example ibuprofen, naproxen)

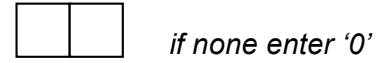

Gels/Creams (for example ibuleve or movelat)

if none enter ' 0 '

Sleeping pills

if none enter ' 0 '

Anti-depressants

if none enter ' 0 '

Other (Please specify) 


\section{Private Treatment}

5. For the last 6 months please detail total treatment costs you paid for yourself; or paid for by private insurance; please do not include any treatments paid for by the NHS. Please round the amounts to the nearest pound.

$\begin{array}{lll}\text { Number } & \text { Medical } & \text { Personal } \\ \text { Of times } & \text { Insurance } & \text { Contribution } \\ & \text { Contribution } & \end{array}$

Private physiotherapist
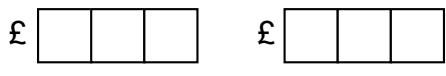

Private hospital specialist (consultant)

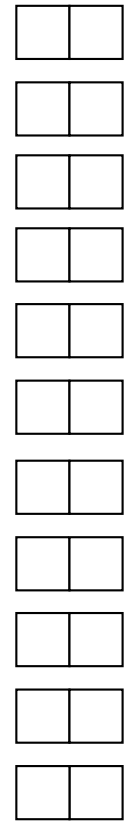

Private osteopath

Private chiropractor

Private psychologist

$£$

$£$

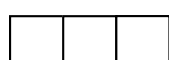

$£$

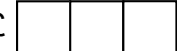

$£$

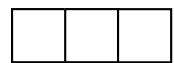

$£$

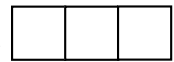

Private counsellor

Private massage therapist

Private aroma therapist

Private acupuncturist

Other (please specify)

Other (please specify)

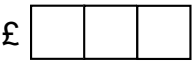

$£$

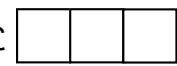

$£$

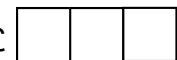

$£$

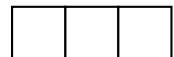

$£$

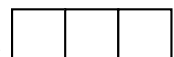

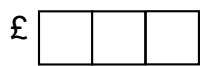
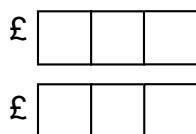

$£$

$£$

$£$

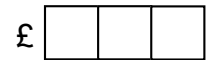

$£$

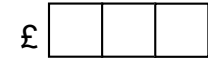

$£$

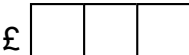

6. In the last 6 months, have you been admitted to a private hospital because of lower back pain?

$$
\begin{array}{ll}
\text { Yes } & \square 1 \\
\text { No } & \square_{2}
\end{array}
$$

If Yes, in total, how many days were you in hospital?

If Yes, what were the total costs paid by your medical insurance? $£$

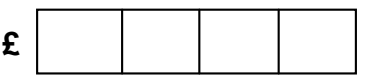

Please give the total costs to the nearest pound*.

If Yes, what were the total costs paid by you?

$£$

Please give the total costs to the nearest pound. *

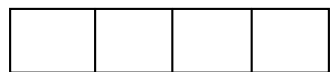

*If you do not know the actual cost please give us your best estimate of the costs. 
7. In the last $\mathbf{6}$ months, have you had any of the following tests in a private hospital in relation to lower back pain?

Number of times

X-ray

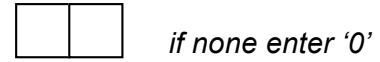

CT Scan

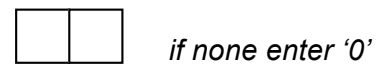

MRI Scan

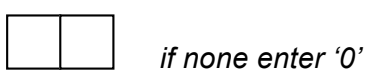

Blood tests (please count all blood tests done on one day as one test)

if none enter ' 0 '

Other (please specify)

if none enter ' 0 '

8. In the last 6 months have you bought (other than by a prescription) any of the following treatments for your back pain? Please estimate the total cost to the nearest pound.

Number Total cost

of times to you

Pain killers

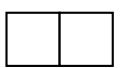

$£$

Anti-inflammatory drugs (for example ibuprofen/nurofen)
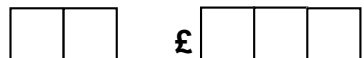

Gels/Creams (for example ibuleve or movelat)
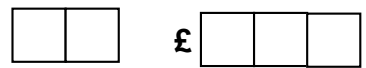

Other (Please specify) 


\section{Products / equipment.}

9. In the last 6 months, have you bought items such as braces or aids, a new bed or mattress, a chair, a massage machine, or any other products or equipment because of your back pain? (please list the item below and estimate the cost to the nearest pound)

\section{Item bought}

1.

. $\mathfrak{£}$

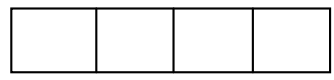

2.

$£$

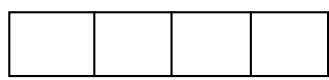

3. $£$

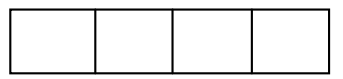

4. $£$

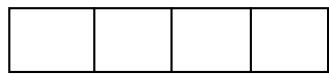

5. $\boldsymbol{\varepsilon}$

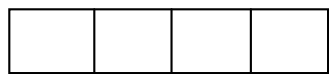

\section{Normal activities.}

10. Over the last 6 months has your back pain stopped you doing your normal activities?

Please cross any that apply and enter the total number of days your back pain stopped you getting on with your normal activities.

Number of days

Employment

Education (i.e. college or University)

Housework

Childcare or care of a relative

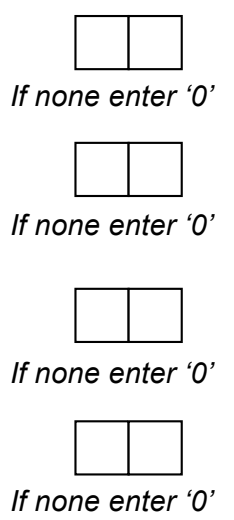




\section{Changes in work status in the last six months}

11. Have you had to take any days off sick from work in the last 6 months due to your low back pain?

$\begin{array}{ll}\text { Yes } & \square{ }_{1} \\ \text { No } & \square_{2} \\ \text { Not applicable } & \square 3\end{array}$

If Yes, how many days?

12. Have you had to change your occupation in the last 6 months due to your back pain?

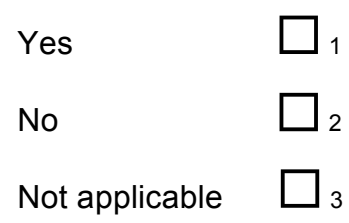

If Yes, what is your new job?

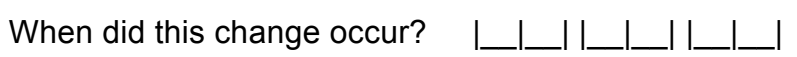

13. Have your hours of employment changed in the last 6 months because of back pain getting better or worse?

$\begin{array}{ll}\text { Yes } & \square_{1} \\ \text { No } & \square_{2} \\ \text { Not applicable } & \square 3\end{array}$

If Yes, by how many hours per week has your employment changed?

When did this change occur?

14. If you were off work when you joined the study, have you returned to work?

$\square$ I am still off work

$\square_{2}$ I returned to work on I_L_ا_L_ا_L_ا_L_ا_L 


\section{Benefits and entitlement to free prescriptions}

15. Do you qualify for free prescriptions?

$\begin{array}{ll}\text { Yes } & \square_{1} \\ \text { No } & \square_{2}\end{array}$

16. Has your benefit status changed in the last 6 months?

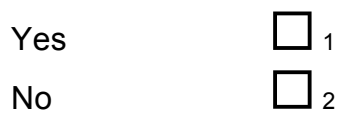

If yes, please cross all benefits that you are currently receiving.

$\begin{array}{lll}\text { Statutory Sick Pay } & \square_{1} \quad \text { Child Tax Credit } \\ \text { Incapacity Benefit } & \square_{2} \quad \text { Council Tax Benefit } \\ \text { Disability living allowance } & \square_{3} \quad \text { Housing Benefit } \\ \text { Disabled persons tax credit } & \square_{4} \quad \text { Attendance allowance } \\ \text { Invalid Care Allowance } & \square_{5} \text { (includes if paid to someone who looks after you) } \\ \text { Other } & \square_{6} \text { please specify } \\ \text { Working Tax Credit } & \square_{7}\end{array}$

THANK YOU FOR COMPLETING THIS QUESTIONNAIRE

If you have any comments, please write them overleaf. 
Your comments: 


\section{Appendix 9}

\section{Core outcome questionnaires}




\section{BeST}

\section{Core Outcome measures for 3 Month questionnaire non-responders}

\section{Participant Study Number:}

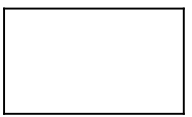

Date of telephone interview:

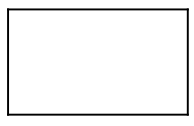

The first questions are about how much your back trouble has been interfering with your daily activities in recent weeks.

1. In the past $\mathbf{4}$ weeks, how much has your back pain interfered with your daily activities on a scale of $0-10$ where 0 is 'no interference' and 10 is 'unable to carry out any activities at all'?
0
12
3
4
56
$\begin{array}{llll}7 & 8 & 9 & 10\end{array}$

2. In the past $\mathbf{4}$ weeks, how much has your back pain changed your ability to take part in

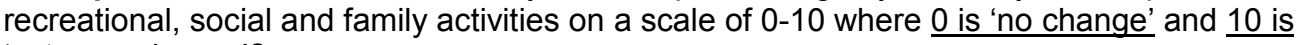
'extreme change'?
01
$2 \quad 3 \quad 4$
5
67
89
10

3. In the past $\mathbf{4}$ weeks, how much has your back pain changed your ability to work (including housework) on a scale of $0-10$ where 0 is 'no change' and 10 is 'extreme change'?
0
1
23
456

4. In the past $\mathbf{4}$ weeks, how bad has your worst back pain been on a scale of 0-10 where 0 is 'no pain' and 10 is 'as bad as a pain could be'?

$$
\begin{array}{llllllllllll}
0 & 1 & 2 & 3 & 4 & 5 & 6 & 7 & 8 & 9 & 10
\end{array}
$$

5. In the past 4 weeks, on average how bad has your back pain been on a scale of $0-10$ where $\underline{0 \text { is }}$ 'no pain' and 10 is 'as bad as a pain could be'?
0
$\begin{array}{llll}2 & 3 & 4 & 5\end{array}$
$6 \quad 7$
$8 \quad 9 \quad 10$

6. How would you rate your back pain today on a scale of $0-10$ where $\underline{0 \text { is 'no pain' and } 10 \text { is 'as }}$ bad as a pain could be'?

$\begin{array}{llllllllllll}0 & 1 & 2 & 3 & 4 & 5 & 6 & 7 & 8 & 9 & 10\end{array}$

7. In what way has your back pain changed in the past three months?

$\begin{array}{llll}\text { Completely recovered } & \square & \text { Much improved } & \square \\ \text { Slightly improved } & \square_{3} & \text { No change } & \square \\ \text { Slightly worsened } & \square & \text { Much worsened } & \square \\ \text { Vastly worsened } & \square & \end{array}$


The next questions are about your views about your health. This information will help keep track of how you feel and how well you are able to do your usual activities.

8. In general, would you say your health is:

Excellent

Very good

Good

Fair

Poor

$\square 1$

$\square 2$

$\square 3$

$\square 4$

$\square 5$

9. The following questions are about activities you might do during a typical day. Does your health now limit you in these activities? If so, how much?

$\begin{array}{ccc}\begin{array}{c}\text { Yes, } \\ \text { limited } \\ \text { a lot }\end{array} & \begin{array}{c}\text { Yes, } \\ \text { limited } \\ \text { a little }\end{array} & \begin{array}{c}\text { No, not limited } \\ \text { at all }\end{array} \\ \square{ }_{1} & \square_{2} & \square_{3} \\ \square \square_{1} & \square_{2} & \square_{3}\end{array}$

a) Moderate activities, such as moving a table, pushing a vacuum cleaner, bowling or playing golf

b) Climbing several flights of stairs

10. During the past 4 weeks, how much of the time have you had any of the following problems with your work or other regular daily activities as a result of your physical health?

$\begin{array}{cccc}\text { All of } & \text { Most of } & \text { Some of } & \text { A little of None of the time } \\ \text { the time the time the time the time }\end{array}$

a) Accomplished less than you would like

$\square_{1}=-\square_{2}=-\square_{3}=2-\square_{4}$

b) Were limited in the kind of work or other activities

$\begin{array}{lllll}\square_{1} & \square_{2} & \square_{3} & \square_{4} & \square_{5} \\ & \square_{5}\end{array}$

11. During the past 4 weeks, how much of the time have you had any of the following problems with your work or other regular activities as a result of any emotional problems (such as feeling depressed or anxious)?

$\begin{array}{cccc}\text { All of } & \text { Most of } & \text { Some of } & \text { A little of None of the time } \\ \text { the time the time } & \text { the time the time }\end{array}$

a) Accomplished less than you would like

b) Were limited in the kind of work or other activities the time the time

12. During the past $\mathbf{4}$ weeks, how much did pain interfere with your normal work (including both work outside the home and housework)?

$\begin{array}{ccccc}\text { Not at all } & \text { A little bit } & \text { Moderately } & \text { Quite a bit } & \text { Extremely } \\ \square_{1} & \square_{2} & \square_{3} & \square_{4} & \square 5\end{array}$


13. These questions are about how you feel and how things have been with you during the past 4 weeks. For each question, please give the one answer that comes closest to the way you have been feeling. How much of the time during the past 4 weeks:

\begin{tabular}{|c|c|c|c|c|c|c|}
\hline \multirow{2}{*}{\multicolumn{2}{|c|}{$\begin{array}{l}\text { Have you felt calm and } \\
\text { peaceful? }\end{array}$}} & $\begin{array}{c}\text { All of } \\
\text { the time }\end{array}$ & $\begin{array}{l}\text { Most of } \\
\text { the time }\end{array}$ & $\begin{array}{l}\text { Some of } \\
\text { the time }\end{array}$ & $\begin{array}{l}\text { A little of } \\
\text { the time }\end{array}$ & $\begin{array}{c}\text { None of the } \\
\text { time }\end{array}$ \\
\hline & & $\square_{1}$ & $\square_{2}$ & $\square_{3}$ & $\square_{4}$ & $\square_{5}$ \\
\hline \multicolumn{2}{|c|}{$\begin{array}{l}\text { Did you have a lot of } \\
\text { energy? }\end{array}$} & $\square_{1}$ & $\square_{2}$ & $\square_{3}$ & $\square_{4}$ & $\square_{5}$ \\
\hline \multicolumn{2}{|c|}{$\begin{array}{l}\text { Have you felt downhearted } \\
\text { and low? }\end{array}$} & $\square_{1}$ & $\square 2$ & $\square_{3}$ & $\square_{4}$ & $\square_{5}$ \\
\hline \multicolumn{7}{|c|}{$\begin{array}{l}\text { During the past } 4 \text { weeks, how much of the time has your physical health OR emotional } \\
\text { oblems interfered with your social activities (like visiting with friends, relatives, etc.)? }\end{array}$} \\
\hline $\begin{array}{l}\text { All of } \\
\text { the time }\end{array}$ & $\begin{array}{l}\text { Most of } \\
\text { the time }\end{array}$ & \multicolumn{2}{|c|}{$\begin{array}{l}\text { Some of } \\
\text { the time }\end{array}$} & \multicolumn{2}{|c|}{$\begin{array}{l}\text { A little of } \\
\text { the time }\end{array}$} & $\begin{array}{l}\text { None of } \\
\text { the time }\end{array}$ \\
\hline$\square 1$ & $\square_{2}$ & \multicolumn{2}{|c|}{$\square_{3}$} & \multicolumn{2}{|c|}{$\square_{4}$} & $\square_{5}$ \\
\hline
\end{tabular}

The following questions are to ask about your general health state at the moment please let me know which statement best describes your own health state today.

\section{Mobility:}

I have no problems in walking about

I have some problems in walking about

I am confined to bed

\section{Self-Care:}

I have no problems with self-care

I have some problems washing or dressing myself

I am unable to wash or dress myself

17. Usual Activities (e.g. work, study, housework, family or leisure activities):

I have no problems with performing my usual activities

I have some problems with performing my usual activities

I am unable to perform my usual activities 


\section{Pain / Discomfort:}

I have no pain or discomfort

I have moderate pain or discomfort

I have extreme pain or discomfort

\section{Anxiety / Depression:}

I am not anxious or depressed

I am moderately anxious or depressed

I am extremely anxious or depressed

\section{Changes in work status in the last three months}

20. Have you had to take any days off sick from work in the last three months due to your low back pain?

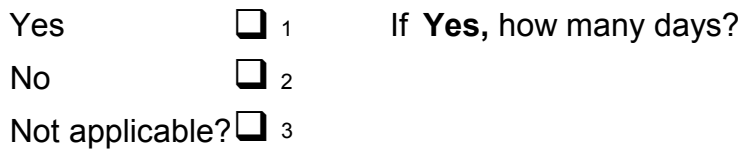

21. Have your hours of employment altered in the last three months because of changes in your back pain?

$\begin{array}{ll}\text { No, stayed the same } & \square_{1} \\ \text { Yes, increased } & \square_{2} \\ \text { Yes, decreased } & \square_{3} \\ \text { Not applicable } & \square_{4}\end{array}$

If Yes, by how many hours per week has your employment changed? When did this change occur?

22. If you were off work when you joined the study, have you returned to work?

$$
\begin{aligned}
& \square_{1} \text { I am still off work } \\
& \square_{2} \text { I returned to work on } \\
& \square_{3} \text { Not applicable? }
\end{aligned}
$$

Advise patient of 6 month questionnaire and importance of completing it and returning it in pre-paid envelope 


\section{BeST}

\section{Core Outcome measures for 6 Month questionnaire non-responders}

\section{Participant Study Number:}

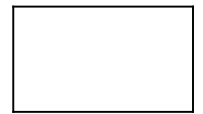

Date of telephone interview:

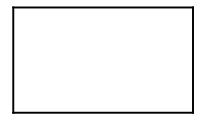

The first questions are about how much your back trouble has been interfering with your daily activities in recent weeks.

1. In the past 4 weeks, how much has your back pain interfered with your daily activities on a scale of $0-10$ where 0 is 'no interference' and 10 is 'unable to carry out any activities at all'?

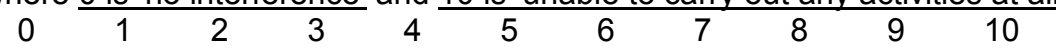

2. In the past $\mathbf{4}$ weeks, how much has your back pain changed your ability to take part in recreational, social and family activities on a scale of $0-10$ where $\underline{0 \text { is 'no change' and } 10 \text { is }}$ 'extreme change'?

$\begin{array}{lllllllllll}0 & 1 & 2 & 3 & 4 & 5 & 6 & 7 & 8 & 9 & 10\end{array}$

3. In the past $\mathbf{4}$ weeks, how much has your back pain changed your ability to work (including housework) on a scale of $0-10$ where 0 is 'no change' and 10 is 'extreme change'?
$0 \quad 1$
23
4
6 ?
$8 \quad 9 \quad 10$

4. In the past $\mathbf{4}$ weeks, how bad has your worst back pain been on a scale of $0-10$ where $\underline{0}$ is 'no pain' and 10 is 'as bad as a pain could be'?

$\begin{array}{lllllllllllll}0 & 1 & 2 & 3 & 4 & 5 & 6 & 7 & 8 & 9 & 10\end{array}$

5. In the past 4 weeks, on average how bad has your back pain been on a scale of $0-10$ where $\underline{0 \text { is }}$

'no pain' and $\frac{10 \text { is 'as bad as a pain could be'? }}{0}$ ?

$\begin{array}{lllll}6 & 7 & 8 & 9 & 10\end{array}$

6. How would you rate your back pain today on a scale of $0-10$ where $\underline{0 \text { is 'no pain' and } 10 \text { is 'as }}$ bad as a pain could be'?

$\begin{array}{lllllllllll}0 & 1 & 2 & 3 & 4 & 5 & 6 & 7 & 8 & 9 & 10\end{array}$

7. How would you describe your back pain compare to when you started the study?

Very much improved (or completely recovered) $\square_{1}$

Minimally (or slightly) improved

Minimally worse

Very much worse
Much improved

No change

Much worse 
The next questions are about your views about your health. This information will help keep track of how you feel and how well you are able to do your usual activities.

8. In general, would you say your health is:

$\begin{array}{ccccc}\text { Excellent } & \text { Very good } & \text { Good } & \text { Fair } & \text { Poor } \\ \square_{1} & \square_{2} & \square_{3} & \square_{4} & \square_{5}\end{array}$

9. The following questions are about activities you might do during a typical day. Does your health now limit you in these activities? If so, how much?

$\begin{array}{ccc}\begin{array}{c}\text { Yes, } \\ \text { limited } \\ \text { a lot }\end{array} & \begin{array}{c}\text { Yes, } \\ \text { limited } \\ \text { a little }\end{array} & \begin{array}{c}\text { No, not limited } \\ \text { at all }\end{array} \\ \square_{1} & \square_{2} & \square_{3} \\ \square_{1} & \square_{2} & \square_{3}\end{array}$

a) Moderate activities, such as moving a table, pushing a vacuum cleaner, bowling or playing golf

b) Climbing several flights of stairs

10. During the past 4 weeks, how much of the time have you had any of the following problems with your work or other regular daily activities as a result of your physical health?

$\begin{array}{cccc}\text { All of } & \text { Most of } & \text { Some of } & \text { A little of None of the time } \\ \text { the time the time } & \text { the time the time }\end{array}$
$\begin{array}{lllll}\text { a) Accomplished less than } & \square_{1} \quad \square_{2} & \square_{3} & \square_{4} & \square \\ \text { you would like }\end{array}$

b) Were limited in the kind of work or other activities

11. During the past 4 weeks, how much of the time have you had any of the following problems with your work or other regular activities as a result of any emotional problems (such as feeling depressed or anxious)?

$\begin{array}{cccc}\text { All of } & \text { Most of } & \text { Some of } & \text { A little of None of the time } \\ \text { the time the time the time } & \text { the time }\end{array}$

a) Accomplished less than $\quad \square_{1} \quad \square_{2} \quad \square_{3} \quad \square_{4} \quad \square 5$ you would like

b) Were limited in the kind of work or other activities

12. During the past $\mathbf{4}$ weeks, how much did pain interfere with your normal work (including both work outside the home and housework)?

$\begin{array}{ccccc}\text { Not at all } & \text { A little bit } & \text { Moderately } & \text { Quite a bit } & \text { Extremely } \\ \square_{1} & \square_{2} & \square_{3} & \square_{4} & \square 5\end{array}$


13. These questions are about how you feel and how things have been with you during the past 4 weeks. For each question, please give the one answer that comes closest to the way you have been feeling. How much of the time during the past 4 weeks:

$\begin{array}{cccc}\text { All of } & \text { Most of } & \text { Some of } & \text { A little of } \\ \text { the time } & \text { the time } & \text { None of the } \\ \text { the time } & \text { the time } & \text { time }\end{array}$

a) Have you felt calm and peaceful? the time the time

the time the time time

b) Did you have a lot of energy?

$\square_{4}$

$\square 5$

c) Have you felt downhearted and low?

$\square 1$

$\square 2$

$\square 3$

$\square 4$

$\square$

14. During the past 4 weeks, how much of the time has your physical health OR emotional problems interfered with your social activities (like visiting with friends, relatives, etc.)?

$\begin{array}{ccccc}\begin{array}{c}\text { All of } \\ \text { the time }\end{array} & \begin{array}{c}\text { Most of } \\ \text { the time }\end{array} & \begin{array}{c}\text { Some of } \\ \text { the time }\end{array} & \begin{array}{c}\text { A little of } \\ \text { the time }\end{array} & \begin{array}{c}\text { None of } \\ \text { the time }\end{array} \\ \square_{1} & \square_{2} & \square_{3} & \square_{4} & \square 5\end{array}$

The following questions are to ask about your general health state at the moment please let me know which statement best describes your own health state today.

\section{Mobility:}

I have no problems in walking about

I have some problems in walking about

I am confined to bed

\section{Self-Care:}

I have no problems with self-care

I have some problems washing or dressing myself

I am unable to wash or dress myself

17. Usual Activities (e.g. work, study, housework, family or leisure activities):

I have no problems with performing my usual activities

I have some problems with performing my usual activities

I am unable to perform my usual activities 


\section{Pain / Discomfort:}

I have no pain or discomfort

I have moderate pain or discomfort

I have extreme pain or discomfort

\section{Anxiety / Depression:}

I am not anxious or depressed

I am moderately anxious or depressed

I am extremely anxious or depressed

\section{Changes in work status in the last three months}

20. Have you had to take any days off sick from work in the last three months due to your low back pain?

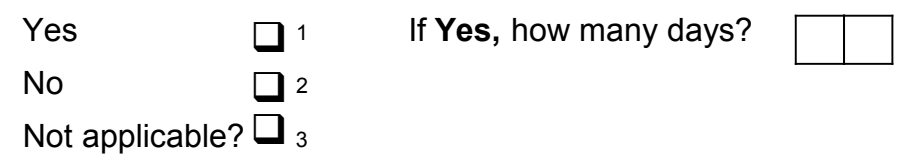

21. Have your hours of employment altered in the last three months because of changes in your back pain?
No, stayed the same
$\square$
Yes, increased
$\square_{2}$
Yes, decreased
$\square_{3}$
Not applicable
$\square_{4}$

If Yes, by how many hours per week has your employment changed?

When did this change occur?

22. If you were off work when you joined the study, have you returned to work?

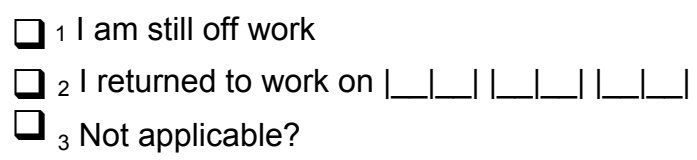

Advise patient of 12 month questionnaire and importance of completing it and returning it in pre-paid envelope 


\section{BeST}

\section{Core Outcome measures for 12 Month questionnaire non-responders}

\section{Participant Study Number:}

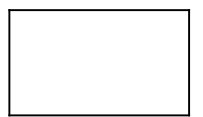

Date of telephone interview:

The first questions are about how much your back trouble has been interfering with your daily activities in recent weeks.

1. In the past $\mathbf{4}$ weeks, how much has your back pain interfered with your daily activities on a scale of $0-10$ where 0 is 'no interference' and 10 is 'unable to carry out any activities at all'?

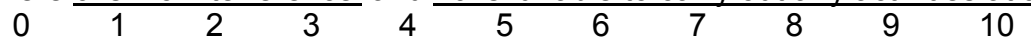

2. In the past 4 weeks, how much has your back pain changed your ability to take part in recreational, social and family activities on a scale of $0-10$ where $\underline{0 \text { is 'no change' and } \underline{10} \text { is }}$ 'extreme change'?

$\begin{array}{lllllllllll}0 & 1 & 2 & 3 & 4 & 5 & 6 & 7 & 8 & 9 & 10\end{array}$

3. In the past 4 weeks, how much has your back pain changed your ability to work (including

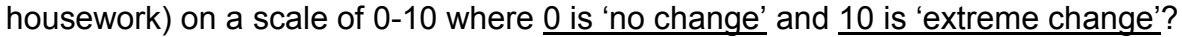

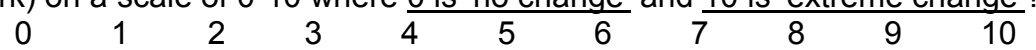

4. In the past $\mathbf{4}$ weeks, how bad has your worst back pain been on a scale of $0-10$ where 0 is 'no pain' and 10 is 'as bad as a pain could be'?

$$
\begin{array}{llllllllllll}
\hline 0 & 1 & 2 & 3 & 4 & 5 & 6 & 7 & 8 & 9 & 10
\end{array}
$$

5. In the past 4 weeks, on average how bad has your back pain been on a scale of $0-10$ where 0 is 'no pain' and 10 is 'as bad as a pain could be'?

$$
\begin{array}{lllllllllll}
0 & 1 & 2 & 3 & 4 & 5 & 6 & 7 & 8 & 9 & 10
\end{array}
$$

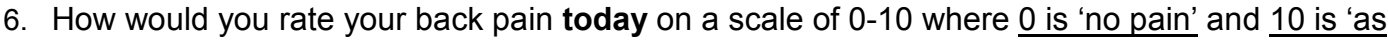
bad as a pain could be'?

$\begin{array}{lllllllllll}0 & 1 & 2 & 3 & 4 & 5 & 6 & 7 & 8 & 9 & 10\end{array}$

7. How would you describe your back pain compare to when you started the study?

Very much improved (or completely recovered) $\square$ Minimally (or slightly) improved

Minimally worse

Very much worse
Much improved

No change

Much worse 
The next questions are about your views about your health. This information will help keep track of how you feel and how well you are able to do your usual activities.

8. In general, would you say your health is:

$\begin{array}{ccccc}\text { Excellent } & \text { Very good } & \text { Good } & \text { Fair } & \text { Poor } \\ \square_{1} & \square_{2} & \square_{3} & \square_{4} & \square_{5}\end{array}$

9. The following questions are about activities you might do during a typical day. Does your health now limit you in these activities? If so, how much?

$\begin{array}{ccc}\begin{array}{c}\text { Yes, } \\ \text { limited } \\ \text { a lot }\end{array} & \begin{array}{c}\text { Yes, } \\ \text { limited } \\ \text { a little }\end{array} & \begin{array}{c}\text { No, not limited } \\ \text { at all }\end{array} \\ \square_{1} & \square_{2} & \square_{3} \\ \square_{1} & \square_{2} & \square_{3}\end{array}$

a) Moderate activities, such as moving a table, pushing a vacuum cleaner, bowling or playing golf

b) Climbing several flights of stairs

10. During the past 4 weeks, how much of the time have you had any of the following problems with your work or other regular daily activities as a result of your physical health?

\begin{tabular}{|c|}
\hline $\begin{array}{c}\text { All of } \\
\text { the time }\end{array}$ \\
\hline
\end{tabular}

a) Accomplished less than you would like

All the time the time

b) Were limited in the kind of work or other activities

11. During the past $\mathbf{4}$ weeks, how much of the time have you had any of the following problems with your work or other regular activities as a result of any emotional problems (such as feeling depressed or anxious)?

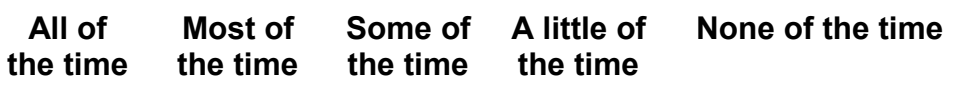

a) Accomplished less than you would like

b) Were limited in the kind of work or other activities

$\begin{array}{lllll}\square_{1} & \square_{2} & \square_{3} & \square_{4} & \square_{5} \\ \square_{1} & \square_{2} & \square_{3} & \square_{4} & \square_{5}\end{array}$

12. During the past $\mathbf{4}$ weeks, how much did pain interfere with your normal work (including both work outside the home and housework)?

$\begin{array}{ccccc}\text { Not at all } & \text { A little bit } & \text { Moderately } & \text { Quite a bit } & \text { Extremely } \\ \square_{1} & \square_{2} & \square_{3} & \square_{4} & \square 5\end{array}$


13. These questions are about how you feel and how things have been with you during the past 4 weeks. For each question, please give the one answer that comes closest to the way you have been feeling. How much of the time during the past 4 weeks:

$\begin{array}{ccccc}\begin{array}{c}\text { All of } \\ \text { the time }\end{array} & \begin{array}{c}\text { Most of } \\ \text { the time }\end{array} & \begin{array}{c}\text { Some of } \\ \text { the time }\end{array} & \begin{array}{c}\text { A little of } \\ \text { the time }\end{array} & \begin{array}{c}\text { None of the } \\ \text { time }\end{array} \\ \square_{1} & \square_{2} & \square_{3} & \square_{4} & \square 5 \\ \square_{1} & \square_{2} & \square_{3} & \square_{4} & \square 5 \\ \square_{1} & \square_{2} & \square_{3} & \square_{4} & \square\end{array}$

Have you felt downhearted and low?

14. During the past 4 weeks, how much of the time has your physical health OR emotional problems interfered with your social activities (like visiting with friends, relatives, etc.)?

$\begin{array}{ccccc}\begin{array}{c}\text { All of } \\ \text { the time }\end{array} & \begin{array}{c}\text { Most of } \\ \text { the time }\end{array} & \begin{array}{c}\text { Some of } \\ \text { the time }\end{array} & \begin{array}{c}\text { A little of } \\ \text { the time }\end{array} & \begin{array}{c}\text { None of } \\ \text { the time }\end{array} \\ \square_{1} & \square_{2} & \square_{3} & \square_{4} & \square_{5}\end{array}$

The following questions are to ask about your general health state at the moment please let me know which statement best describes your own health state today.

15. Mobility:

I have no problems in walking about

I have some problems in walking about

I am confined to bed

\section{Self-Care:}

I have no problems with self-care

I have some problems washing or dressing myself

I am unable to wash or dress myself

17. Usual Activities (e.g. work, study, housework, family or leisure activities):

I have no problems with performing my usual activities

I have some problems with performing my usual activities

I am unable to perform my usual activities 


\section{Pain / Discomfort:}

I have no pain or discomfort

I have moderate pain or discomfort

I have extreme pain or discomfort

\section{Anxiety / Depression:}

I am not anxious or depressed

I am moderately anxious or depressed

I am extremely anxious or depressed

\section{Changes in work status in the last three months}

20. Have you had to take any days off sick from work in the last three months due to your low back pain?

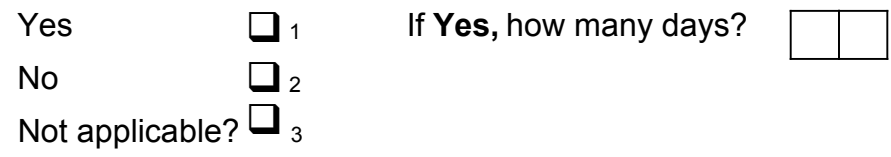

21. Have your hours of employment altered in the last three months because of changes in your back pain?

$\begin{array}{ll}\text { No, stayed the same } & \square_{1} \\ \text { Yes, increased } & \square_{2} \\ \text { Yes, decreased } & \square{ }_{3} \\ \text { Not applicable } & \square_{4}\end{array}$

If Yes, by how many hours per week has your employment changed?

When did this change occur?

22. If you were off work when you joined the study, have you returned to work?

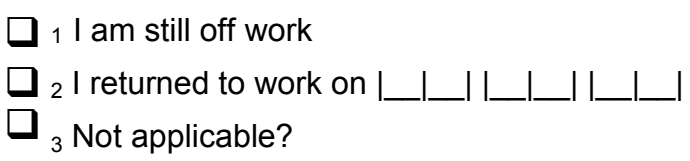

Thank patient for taking part in the study and advise that this Was their final questionnaire 



\section{Appendix 10}

Event notification form 


\section{BEST Study, Event notification form}
${ }^{\star \star} T e l e p h o n e$ Warwick immediately with any notification of withdrawal, death, serious adverse event or complaint ${ }^{\star \star}$

Emma Withers on 02400000000

Completed by Date of completion ....................

\section{Participant details:}

Participant ID number

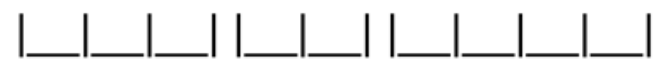

Date of birth

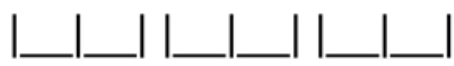

${ }^{* *}$ Only include the participant's name or address if used to notify a name or address change

Old name

Old address
New name

New address
Back Skills Training (BeST) Trial

The University of Warwick Centre for Primary Health Care

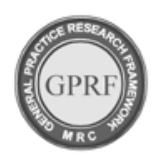

MRC General Practice Research Framework 


\section{Patient request for withdrawal from *treatment* (see also next item '2')}

Date request received

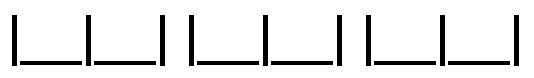

Reason for request (if given)

Date Warwick informed

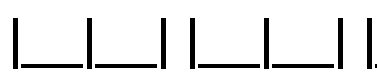

Phone Emma at

Warwick as soon as possible with this information

\section{Patient request for withdrawal from *follow-up* (i.e., from receiving} questionnaires)

Date request received

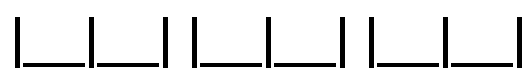

Reason for request (if given)

Date Warwick informed

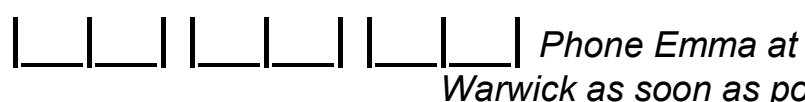

Warwick as soon as possible with this information

3. Practice request for patient to be withdrawn from *follow-up* (i.e., from receiving questionnaires)

Date request received

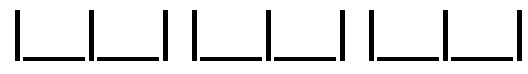

Reason for request (if given)

Date Warwick informed

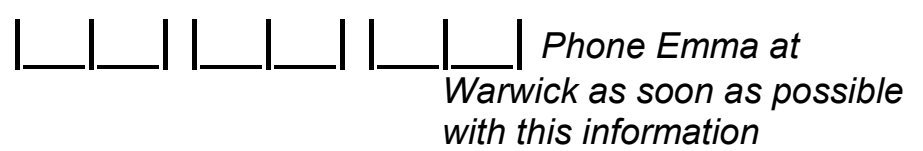
with this information 


\section{Death notification}

Date of death

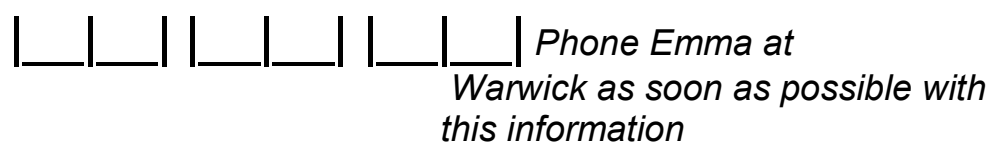

Cause of death (if known). this information

Date Warwick informed

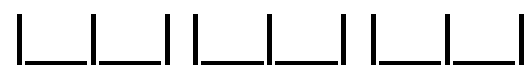

Message taken by (at Warwick)

\section{Serious adverse event notification}

Date of event

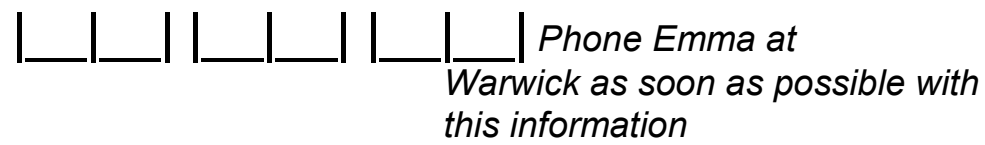

Nature of possible adverse event.

Date Warwick informed

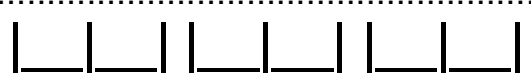

Source of notification of possible adverse event.

Message taken by (at Warwick)

\section{Complaint notification}

Date of notification

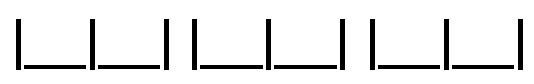

Nature of complaint

Date Warwick informed

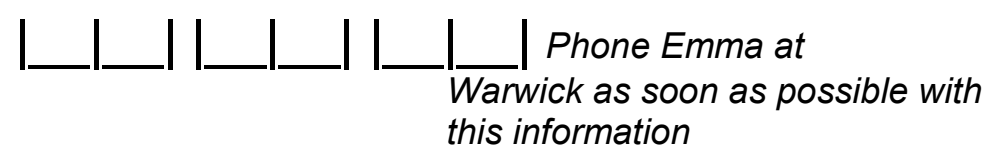

Source of notification of complaint.

Message taken by (at Warwick)

Completed by

(Block capitals)

Date of completion

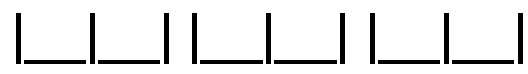




\section{Pregnancy}

Completed by

(Block capitals)

Date of completion

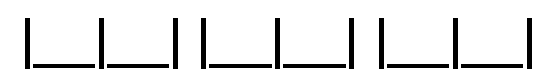

\section{Participant details}

Participant ID number

Date of birth

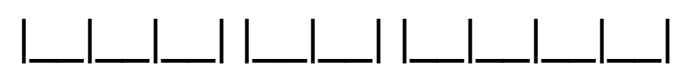

Due date
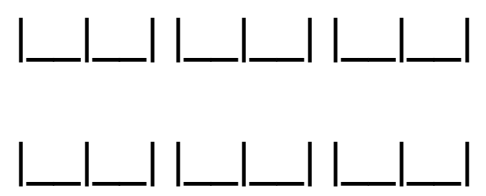



\section{Appendix II}

\section{Demography and baseline assessments}




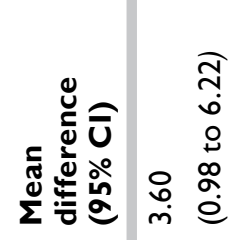

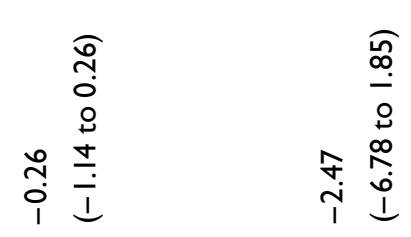

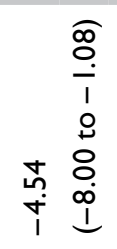

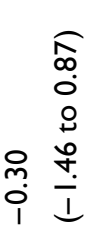

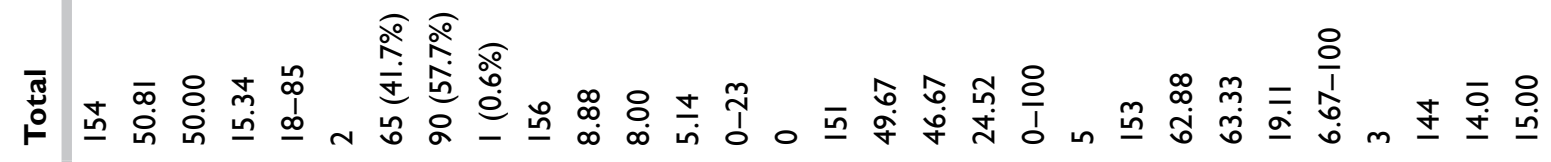

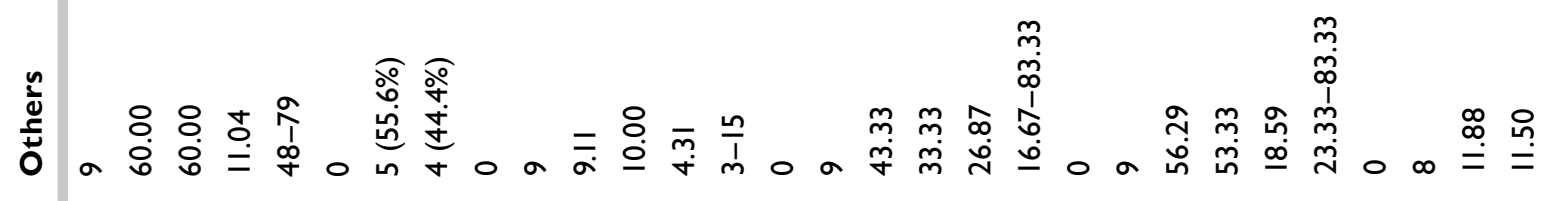

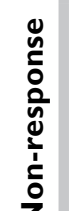

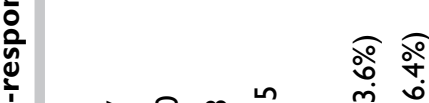

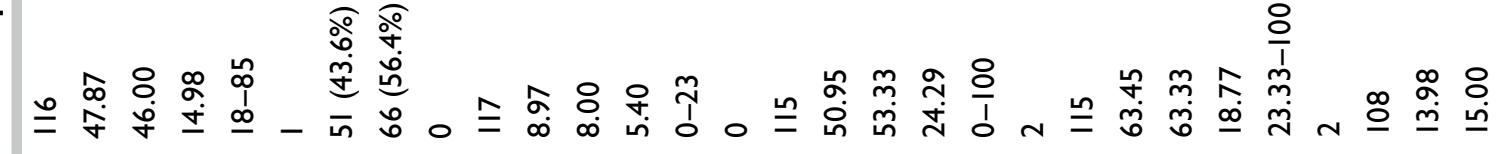

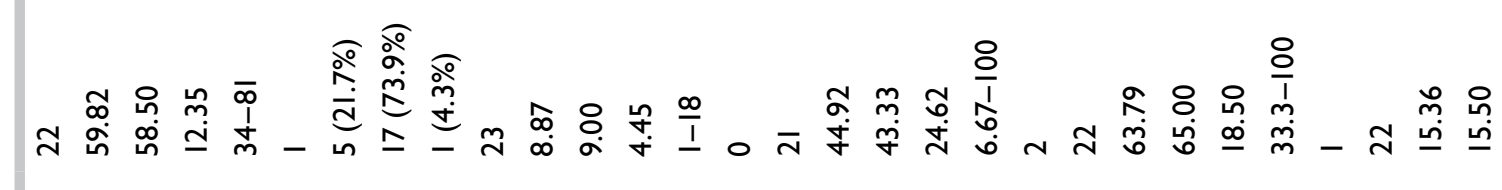

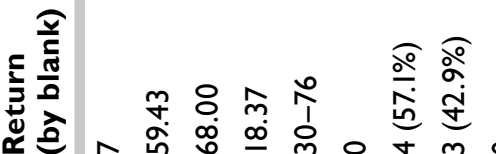

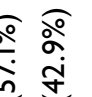

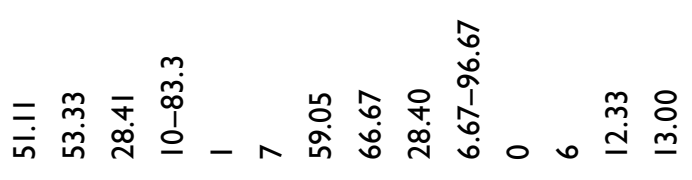

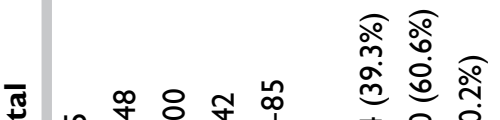

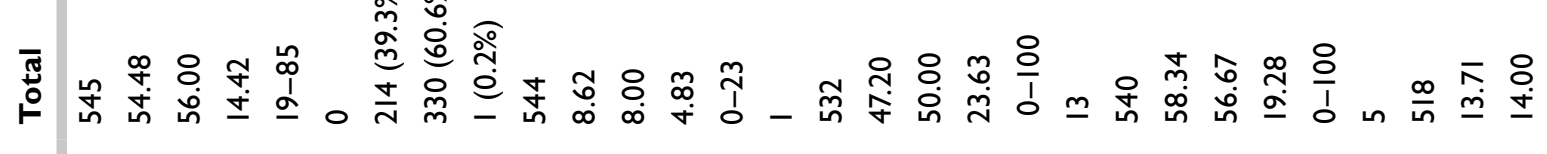

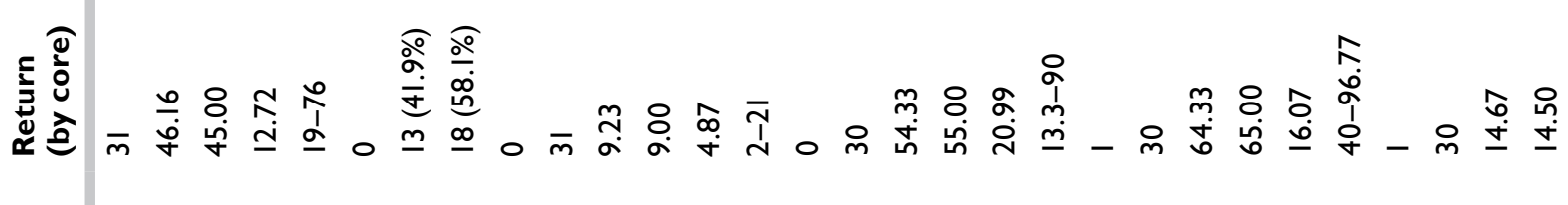

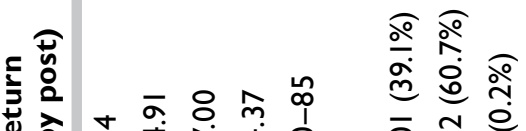

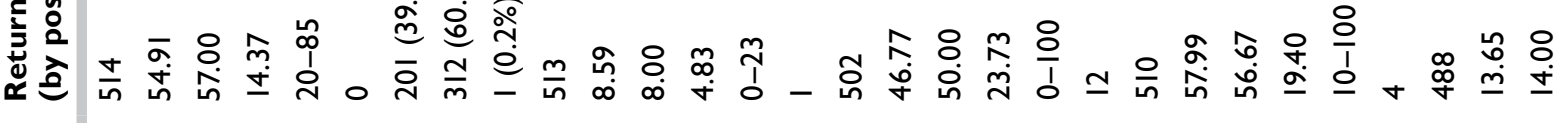

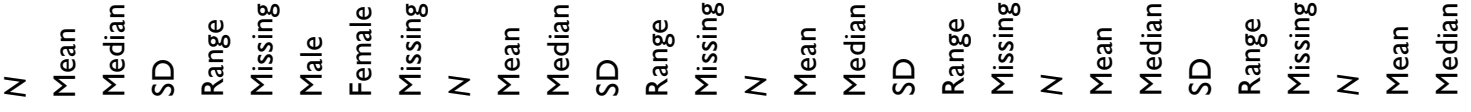




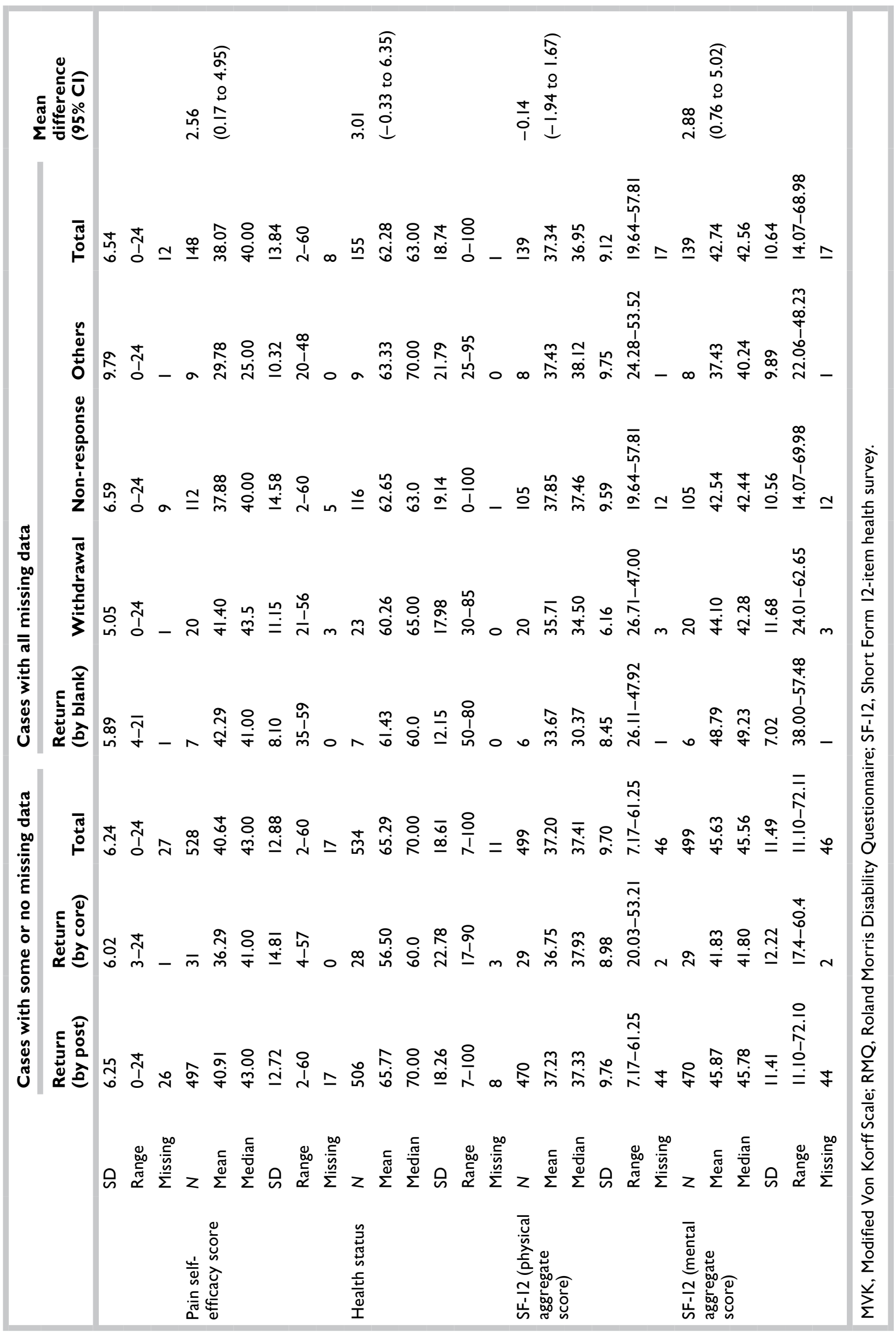




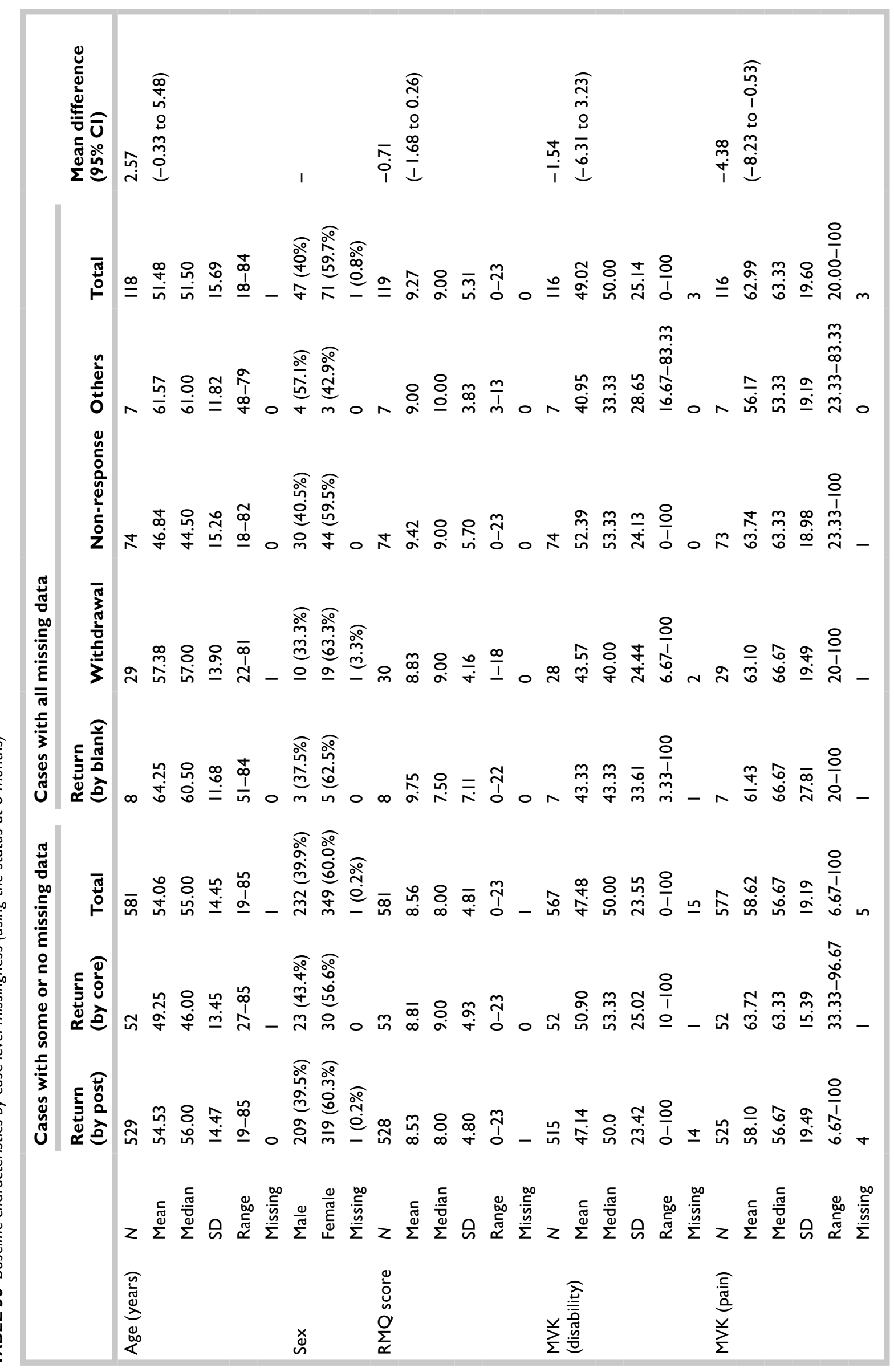




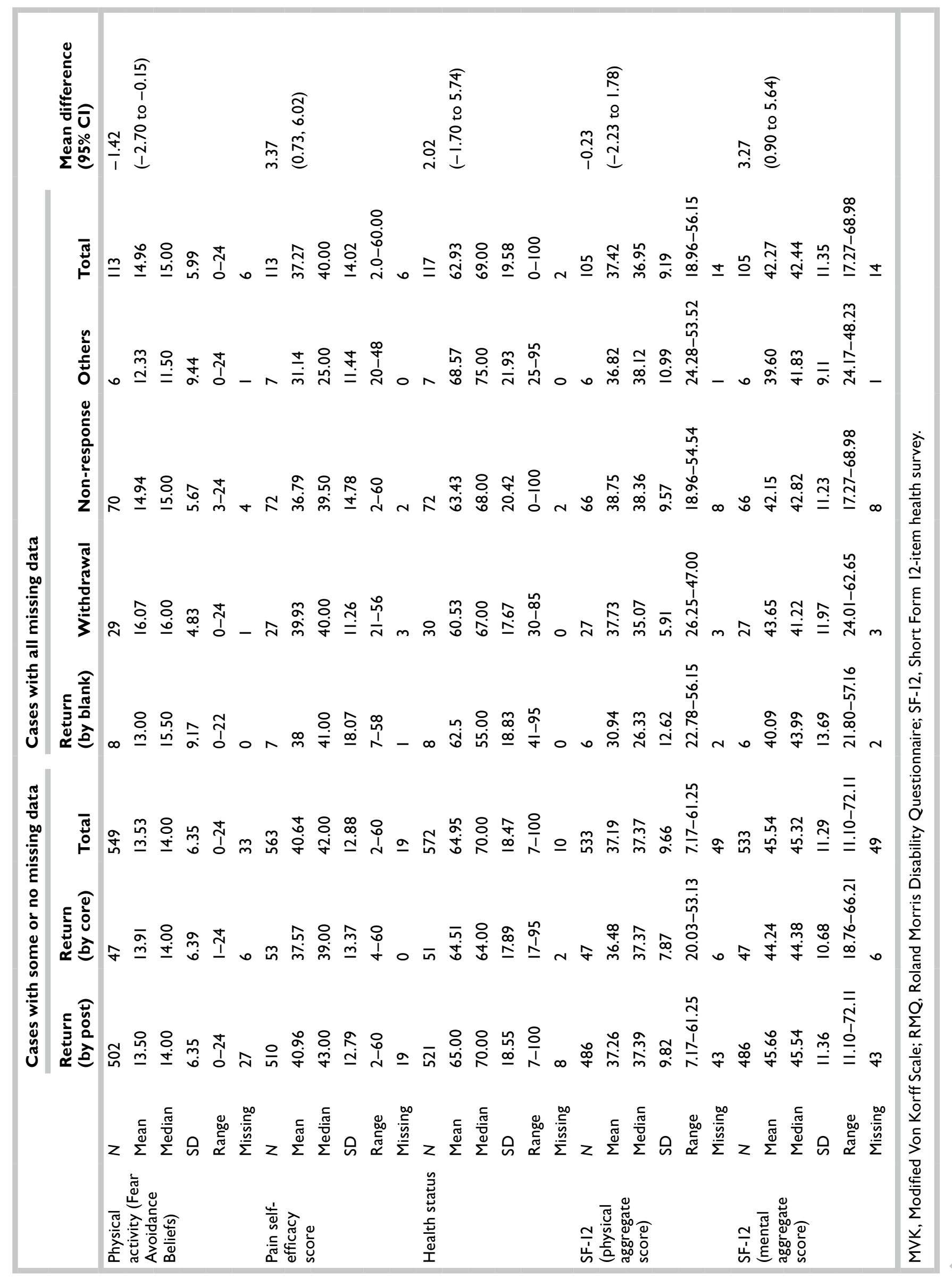



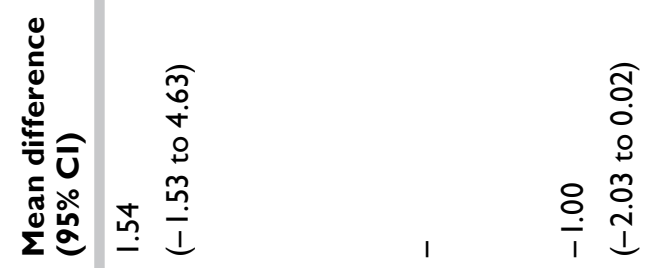

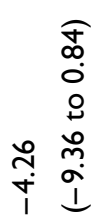

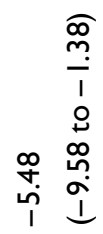

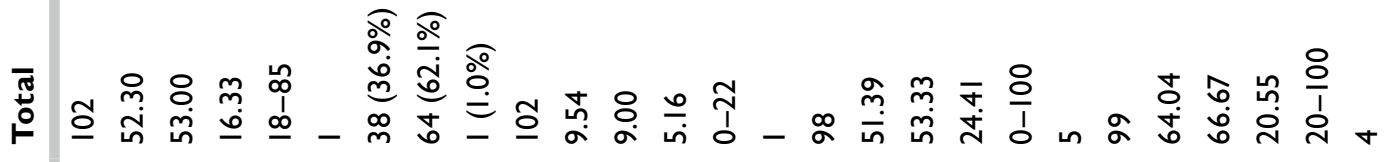

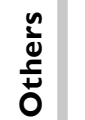

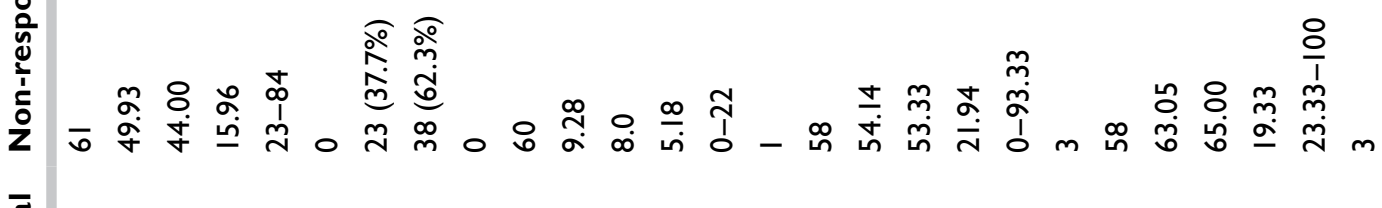

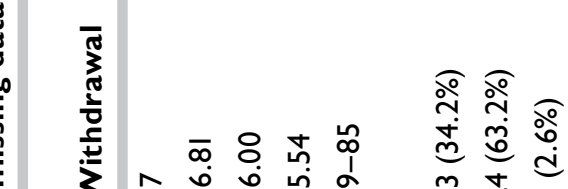

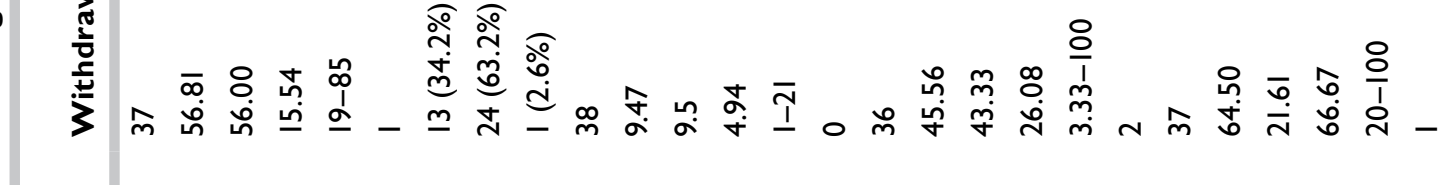

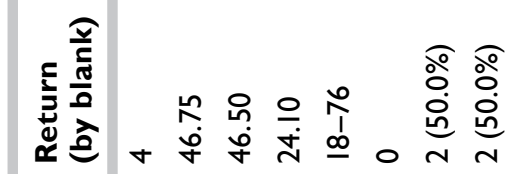

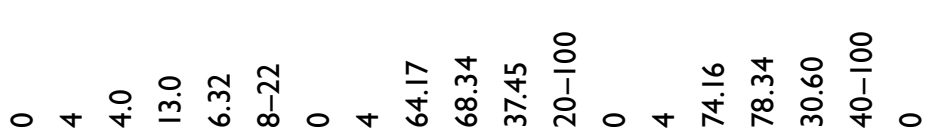

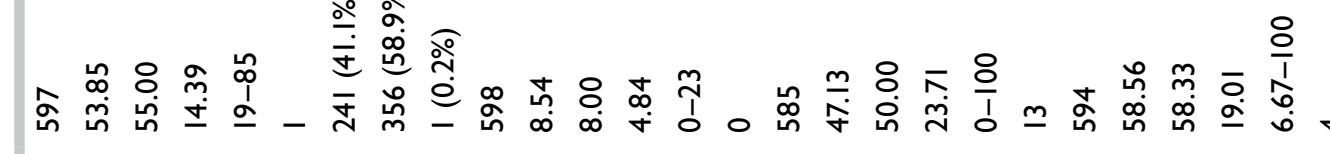

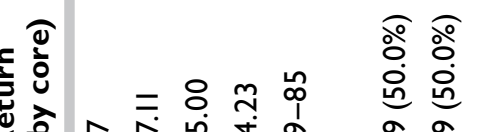

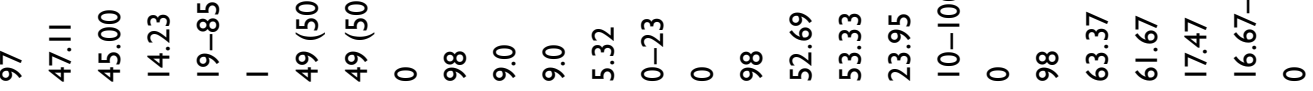

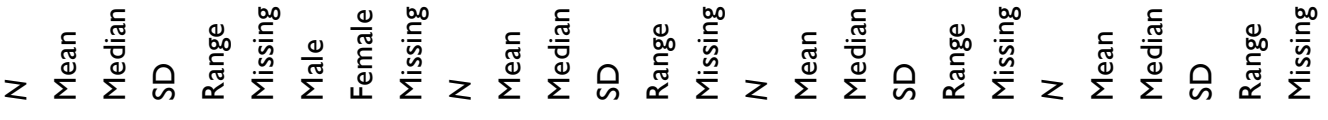

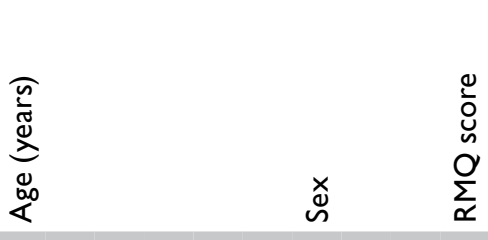




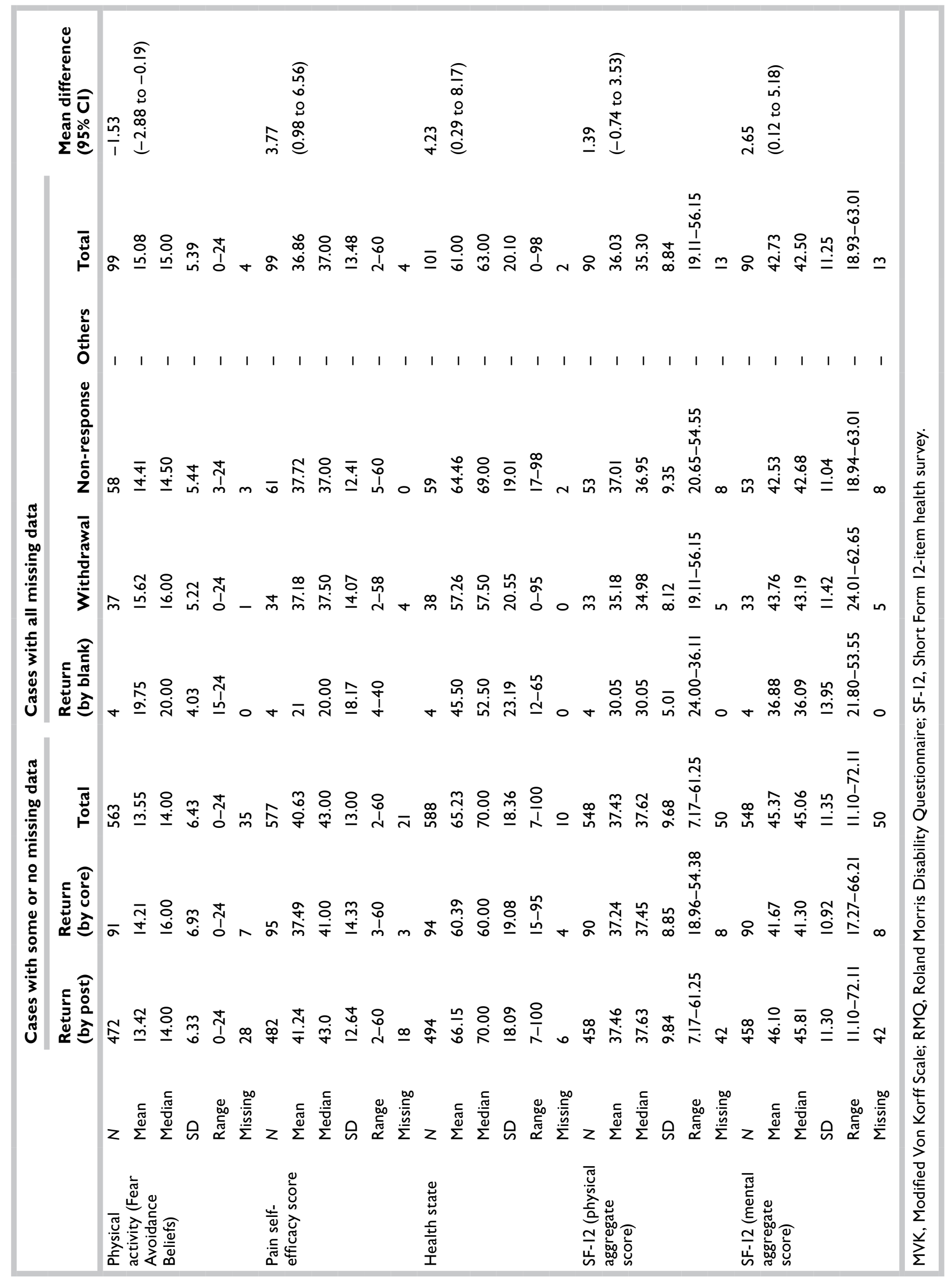





\section{Appendix 12}

\section{Missing data and characteristics of the randomised sample}



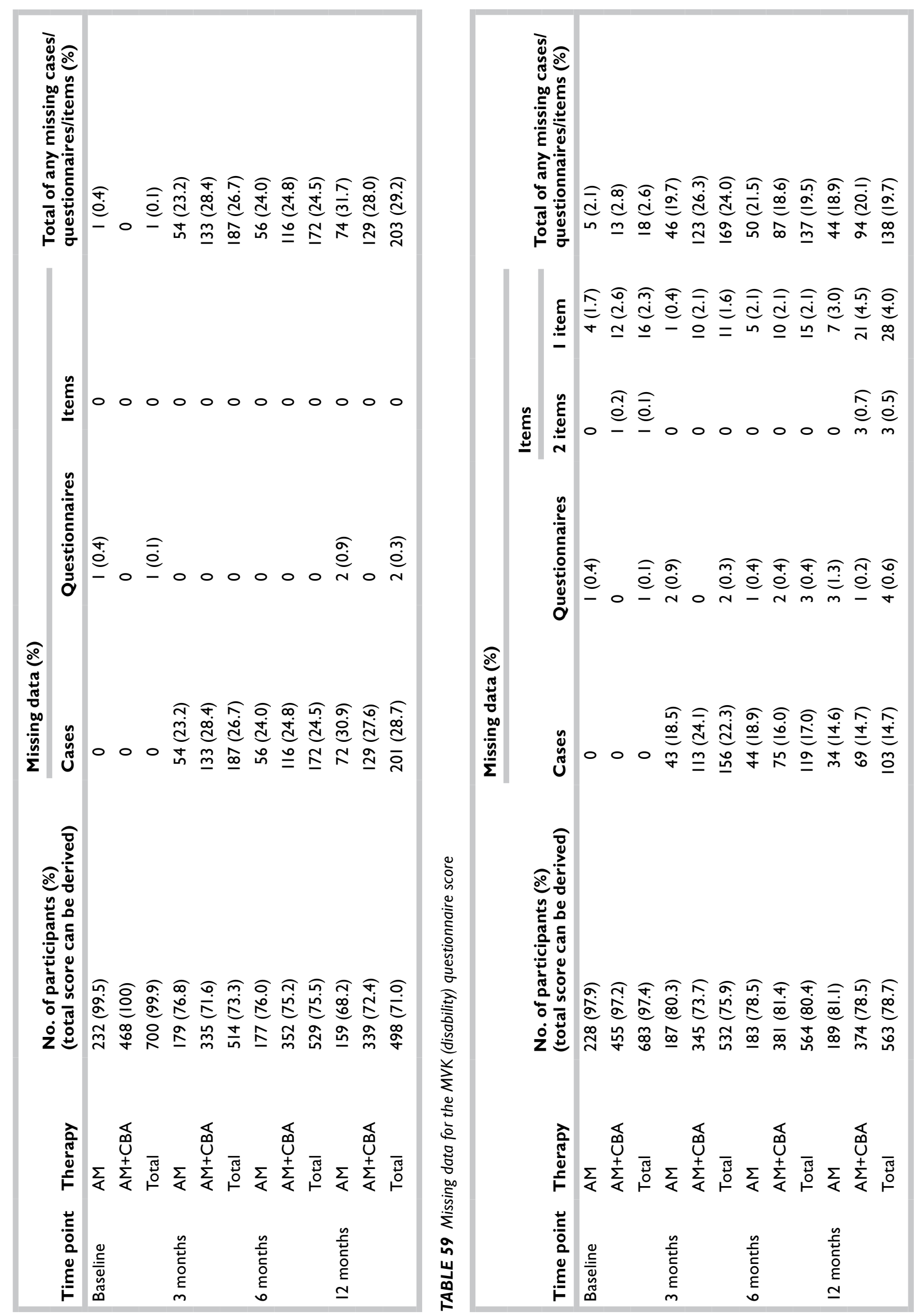

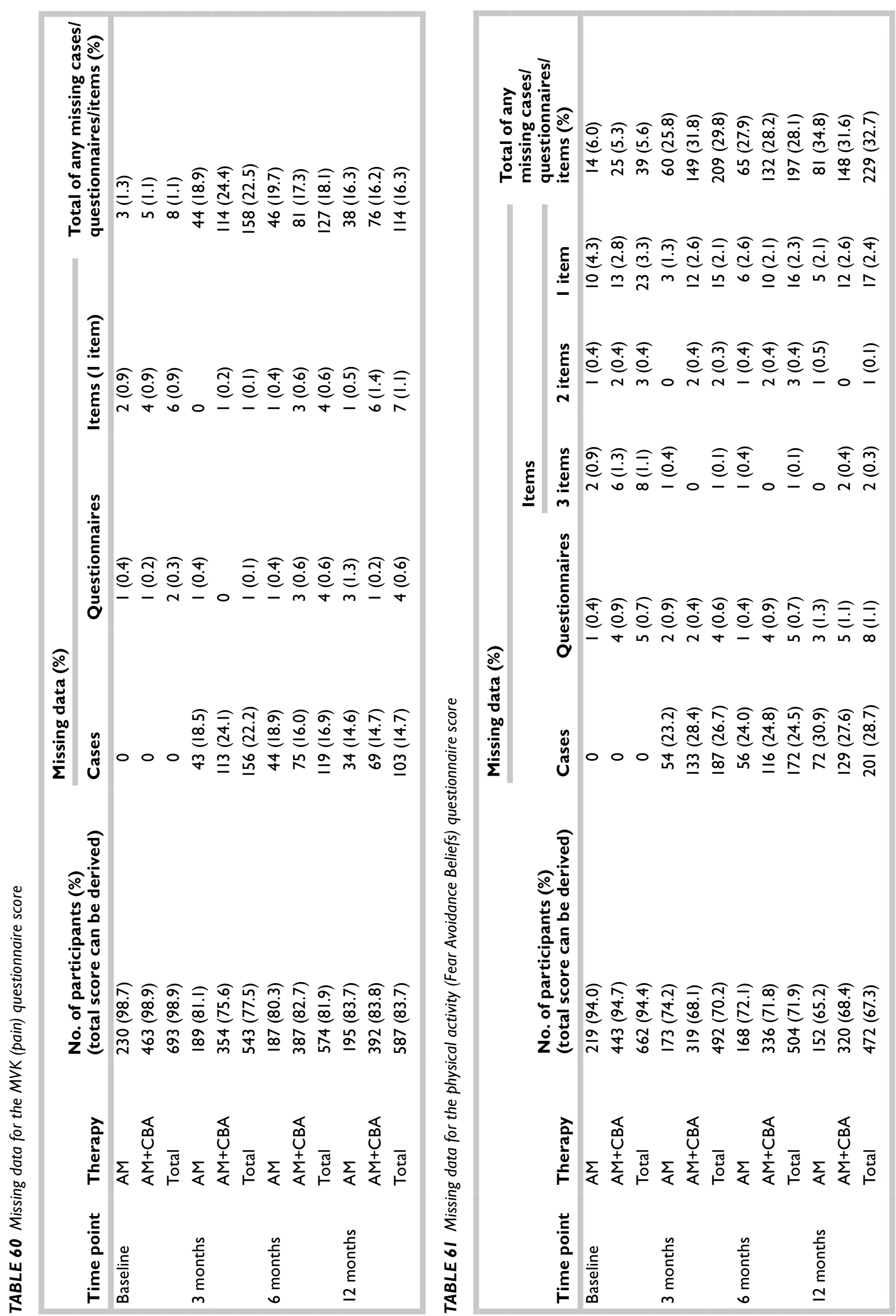


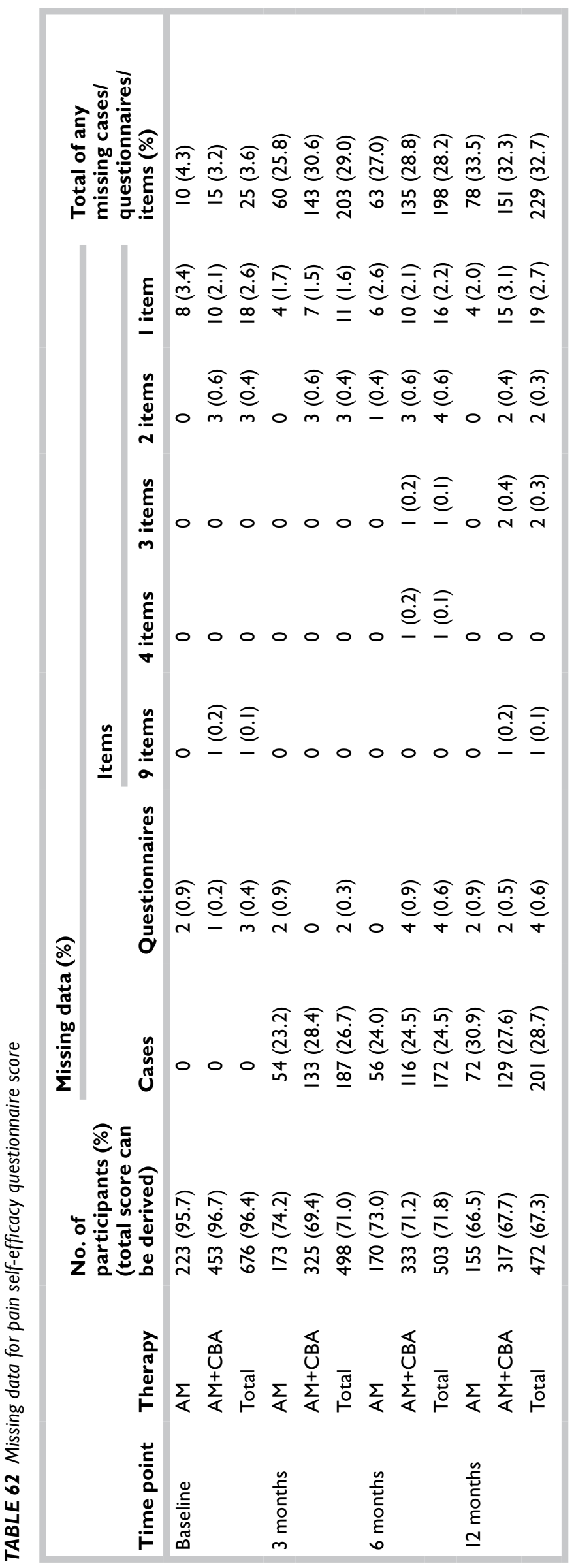



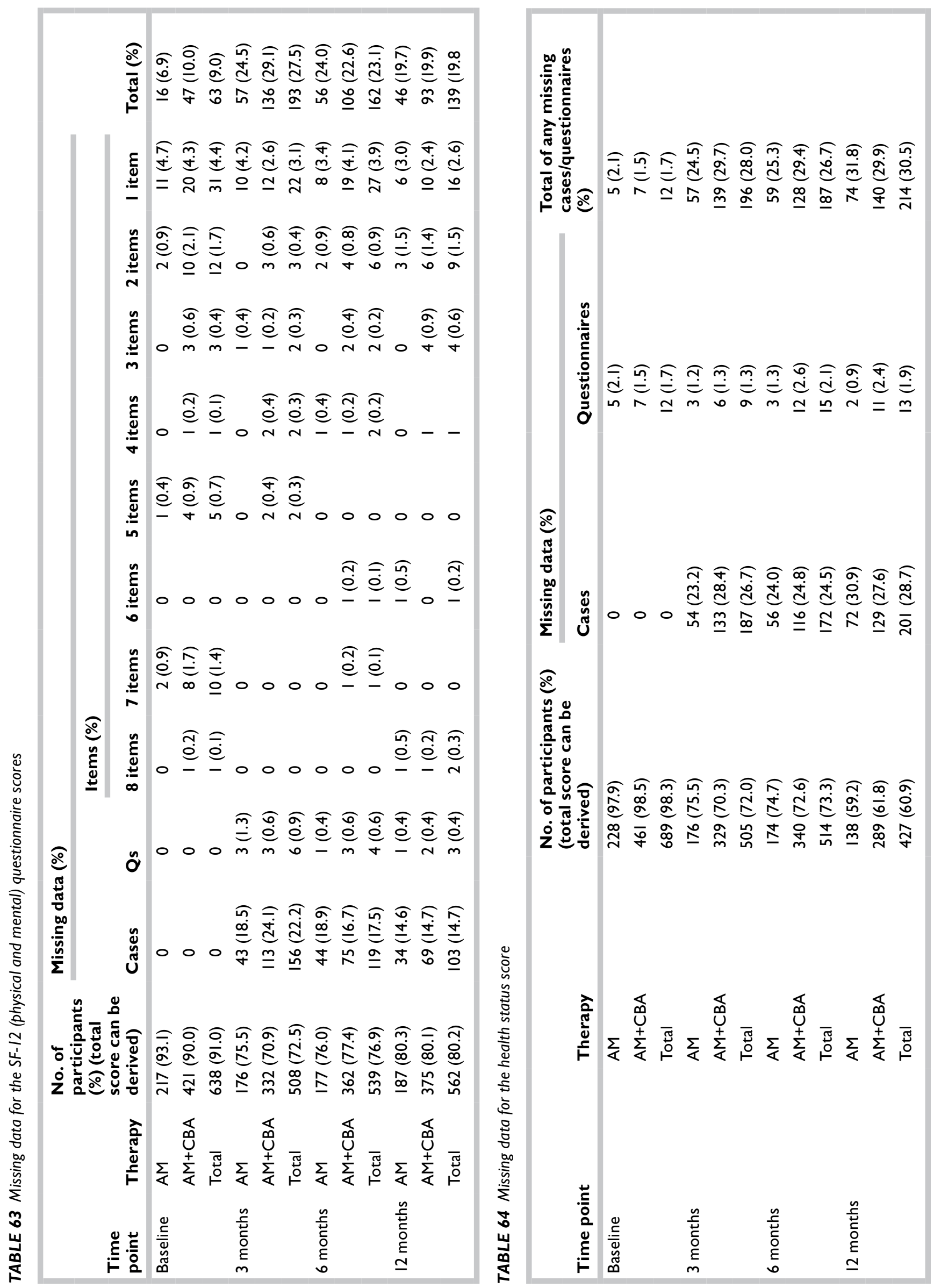



\section{Appendix 13 \\ Data on employment, sickness \\ and benefit status}

TABLE 65 Hours of employment altered

\begin{tabular}{|c|c|c|c|c|c|}
\hline & Month & & AM & $A M+C B A$ & $p$-value ${ }^{a}$ \\
\hline \multirow{15}{*}{$\begin{array}{l}\text { Hours of employment } \\
\text { altered }\end{array}$} & 3 & No & 77 (33.1\%) & I5I (32.3\%) & 0.6973 \\
\hline & & Yes, increased & I (0.4\%) & I (0.2\%) & \\
\hline & & Yes, decreased & 5 (2.2\%) & 7 (I.5\%) & \\
\hline & & Not applicable & 93 (39.9\%) & 173 (37.0\%) & \\
\hline & & Missing & 57 (24.5\%) & $136(29.1 \%)$ & \\
\hline & 6 & No & $12(5.2 \%)$ & $33(7.1 \%)$ & 0.2860 \\
\hline & & Yes, increased & 68 (29.2\%) & 159 (34.0\%) & \\
\hline & & Yes, decreased & 92 (39.5\%) & 157 (33.6\%) & \\
\hline & & Not applicable & $8(3.4 \%)$ & $24(5.1 \%)$ & \\
\hline & & Missing & $53(22.8 \%)$ & 95 (20.3\%) & \\
\hline & 12 & No & $23(9.9 \%)$ & $36(7.7 \%)$ & 0.5627 \\
\hline & & Yes, increased & 69 (29.6\%) & $163(34.8 \%)$ & \\
\hline & & Yes, decreased & $82(35.2 \%)$ & $148(31.6 \%)$ & \\
\hline & & Not applicable & II (4.7\%) & $26(5.6 \%)$ & \\
\hline & & Missing & $48(20.6 \%)$ & $95(20.3 \%)$ & \\
\hline \multirow[t]{18}{*}{ If yes: } & 3 & $N$ & 6 & 8 & \\
\hline & & Mean & 9.5 & 10.1 & \\
\hline & & SD & 8.0 & 6.9 & \\
\hline & & Median & 7.5 & 9.0 & \\
\hline & & Range & $3-25$ & $2-23$ & \\
\hline & & Missing & 227 & 460 & \\
\hline & 6 & $N$ & 3 & 10 & \\
\hline & & Mean & 13 & 10.8 & \\
\hline & & SD & 10.1 & 7.1 & \\
\hline & & Median & 15.0 & 10.0 & \\
\hline & & Range & $2-22$ & $0-20$ & \\
\hline & & Missing & 230 & 458 & \\
\hline & 12 & $N$ & 1 & 8 & \\
\hline & & Mean & 50 & 9.6 & \\
\hline & & SD & - & 2.9 & \\
\hline & & Median & 50 & 10.0 & \\
\hline & & Range & 50 & $5-14$ & \\
\hline & & Missing & 232 & 460 & \\
\hline
\end{tabular}


TABLE 66 Days off sick

\begin{tabular}{|c|c|c|c|c|c|}
\hline & Month & & AM & $A M+C B A$ & $p$-value \\
\hline \multirow{12}{*}{$\begin{array}{l}\text { Days off sick from } \\
\text { work }\end{array}$} & \multirow[t]{4}{*}{3} & Yes & 18 (7.7\%) & $30(6.4 \%)$ & \multirow[t]{4}{*}{0.1987} \\
\hline & & No & 69 (29.6\%) & $153(32.7 \%)$ & \\
\hline & & Not applicable & 94 (40.3\%) & $156(33.3 \%)$ & \\
\hline & & Missing & $52(22.3 \%)$ & 129 (27.6\%) & \\
\hline & \multirow[t]{4}{*}{6} & Yes & $13(5.6 \%)$ & 34 (7.3\%) & \multirow[t]{4}{*}{0.6366} \\
\hline & & No & 75 (32.2\%) & 175 (37.4\%) & \\
\hline & & Not applicable & 94 (40.3\%) & $166(35.5 \%)$ & \\
\hline & & Missing & 51 (21.9\%) & 93 (19.9\%) & \\
\hline & \multirow[t]{4}{*}{12} & Yes & $29(12.5 \%)$ & 37 (7.9\%) & \multirow[t]{4}{*}{0.0818} \\
\hline & & No & 69 (29.6\%) & 177 (37.8\%) & \\
\hline & & Not applicable & 89 (38.2\%) & $167(35.7 \%)$ & \\
\hline & & Missing & 46 (19.7\%) & 87 (18.6\%) & \\
\hline \multirow[t]{18}{*}{ No. of days sick } & \multirow[t]{6}{*}{3} & $N$ & 17 & 30 & \\
\hline & & Mean & 12.2 & 15.6 & \multirow[t]{6}{*}{0.8754} \\
\hline & & SD & 21.4 & 25.2 & \\
\hline & & Median & 5.0 & 4.0 & \\
\hline & & Range & $\mathrm{I}-90$ & $1-92$ & \\
\hline & & Missing & 216 & 438 & \\
\hline & \multirow[t]{6}{*}{6} & $N$ & 12 & 29 & \\
\hline & & Mean & 6.1 & 19.1 & \multirow[t]{6}{*}{0.5110} \\
\hline & & SD & 4.4 & 25.8 & \\
\hline & & Median & 5.5 & 7.0 & \\
\hline & & Range & $0-14$ & $1-90$ & \\
\hline & & Missing & 221 & 439 & \\
\hline & \multirow[t]{6}{*}{12} & $N$ & 27 & 33 & \\
\hline & & Mean & 10.2 & 24.9 & \multirow[t]{5}{*}{0.8979} \\
\hline & & SD & 12.9 & 46.5 & \\
\hline & & Median & 5.0 & 4.0 & \\
\hline & & Range & $1-49$ & $0-180$ & \\
\hline & & Missing & 206 & 435 & \\
\hline \multirow[t]{9}{*}{ Benefit status } & \multirow[t]{3}{*}{3} & Yes & II (4.7\%) & $28(6.0 \%)$ & \\
\hline & & No & 155 (66.5\%) & 276 (59.0\%) & \\
\hline & & Missing & $67(28.8 \%)$ & $164(35.0 \%)$ & \\
\hline & \multirow[t]{3}{*}{6} & Yes & 8 (3.4\%) & 24 (5.1\%) & \\
\hline & & No & I58 (67.8\%) & $312(66.7 \%)$ & \\
\hline & & Missing & $67(28.8 \%)$ & $132(28.2 \%)$ & \\
\hline & \multirow[t]{3}{*}{12} & Yes & 7 (3.0\%) & $18(3.9 \%)$ & \\
\hline & & No & 132 (56.7\%) & 277 (59.2\%) & \\
\hline & & Missing & 94 (40.3\%) & $173(37.0 \%)$ & \\
\hline
\end{tabular}


TABLE 67 Benefit and entitlement status - 3 months

\begin{tabular}{|c|c|c|c|}
\hline & & AM (\%) & $A M+C B A(\%)$ \\
\hline \multirow[t]{3}{*}{ Statutory Sick Pay } & No & $34(14.6)$ & $65(13.9)$ \\
\hline & Yes & I (0.4) & $2(0.4)$ \\
\hline & Missing & $198(85)$ & $401(85.7)$ \\
\hline \multirow[t]{3}{*}{ Incapacity Benefit } & No & $26(11.2)$ & $54(11.5)$ \\
\hline & Yes & $9(3.9)$ & $12(2.6)$ \\
\hline & Missing & $198(85)$ & $402(85.9)$ \\
\hline \multirow[t]{3}{*}{ Disability Living Allowance } & No & $26(I I .2)$ & $44(9.4)$ \\
\hline & Yes & $9(3.9)$ & $23(4.9)$ \\
\hline & Missing & $198(85)$ & $40 \mathrm{I}(85.7)$ \\
\hline \multirow[t]{3}{*}{ Severe Disablement Allowance } & No & $34(14.6)$ & $65(13.9)$ \\
\hline & Yes & I (0.4) & I (0.2) \\
\hline & Missing & $198(85)$ & $402(85.9)$ \\
\hline \multirow[t]{3}{*}{ Disabled Persons Tax Credit } & No & $35(15.0)$ & $66(14.1)$ \\
\hline & Yes & 0 & 0 \\
\hline & Missing & $198(85.0)$ & $402(85.9)$ \\
\hline \multirow[t]{3}{*}{ Carer's Allowance } & No & $34(14.6)$ & $61(13.0)$ \\
\hline & Yes & I (0.4) & $5(1.1)$ \\
\hline & Missing & $198(85)$ & $402(85.9)$ \\
\hline \multirow[t]{3}{*}{ Jobseeker's Allowance } & No & $32(13.7)$ & $63(13.5)$ \\
\hline & Yes & $3(1.3)$ & $3(0.6)$ \\
\hline & Missing & $198(85.0)$ & 402 (85.9) \\
\hline \multirow[t]{3}{*}{ Income Support } & No & $31(13.3)$ & $53(11.3)$ \\
\hline & Yes & $4(1.7)$ & $14(3.0)$ \\
\hline & Missing & $198(85)$ & $40 \mathrm{I}(85.7)$ \\
\hline \multirow[t]{3}{*}{ Working Tax Credit } & No & $31(13.3)$ & $64(13.7)$ \\
\hline & Yes & $4(1.7)$ & $2(0.4)$ \\
\hline & Missing & $198(85)$ & 402 (85.9) \\
\hline \multirow[t]{3}{*}{ Child Tax Credit } & No & $31(13.3)$ & $58(12.4)$ \\
\hline & Yes & $4(1.7)$ & $8(1.7)$ \\
\hline & Missing & $198(85.0)$ & 402 (85.9) \\
\hline \multirow[t]{3}{*}{ Council Tax Benefit } & No & $20(8.6)$ & $47(10.0)$ \\
\hline & Yes & $15(6.4)$ & $19(4.1)$ \\
\hline & Missing & $198(85.0)$ & $402(85.9)$ \\
\hline \multirow[t]{3}{*}{ Housing Benefit } & No & $26(11.2)$ & $51(10.9)$ \\
\hline & Yes & $9(3.9)$ & $15(3.2)$ \\
\hline & Missing & $198(85.0)$ & $402(85.9)$ \\
\hline \multirow[t]{3}{*}{ Attendance Allowance } & No & $32(13.7)$ & $58(12.4)$ \\
\hline & Yes & $3(1.3)$ & $8(1.7)$ \\
\hline & Missing & $198(85.0)$ & 402 (85.9) \\
\hline \multirow[t]{3}{*}{ Pension Credit } & No & $25(10.7)$ & $55($ (II.8) \\
\hline & Yes & $10(4.3)$ & II (2.4) \\
\hline & Missing & $198(85.0)$ & $402(85.9)$ \\
\hline \multirow[t]{3}{*}{ Other } & No & 31 (13.3) & $57(12.2)$ \\
\hline & Yes & $4(1.7)$ & $9(1.9)$ \\
\hline & Missing & $198(85.0)$ & 402 (85.9) \\
\hline
\end{tabular}


TABLE 68 Benefit and entitlement status - 6 months

\begin{tabular}{|c|c|c|c|}
\hline & & $A M(\%)$ & AM+CBA (\%) \\
\hline \multirow[t]{3}{*}{ Statutory Sick Pay } & No & $40(17.2)$ & $68(14.5)$ \\
\hline & Yes & $2(0.9)$ & II (2.4) \\
\hline & Missing & $191(82.0)$ & $389(83.1)$ \\
\hline \multirow[t]{3}{*}{ Incapacity Benefit } & No & $31(13.3)$ & $64(13.7)$ \\
\hline & Yes & II (4.7) & $15(3.2)$ \\
\hline & Missing & $191(82.0)$ & $389(83.1)$ \\
\hline \multirow[t]{3}{*}{ Disability Living Allowance } & No & $32(13.7)$ & $61(13.0)$ \\
\hline & Yes & $10(4.3)$ & $18(3.9)$ \\
\hline & Missing & $191(82.0)$ & $389(83.1)$ \\
\hline \multirow[t]{3}{*}{ Severe Disablement Allowance } & No & $42(18.0)$ & $79(16.9)$ \\
\hline & Yes & 0 & 0 \\
\hline & Missing & $191(82.0)$ & $389(83.1)$ \\
\hline \multirow[t]{3}{*}{ Disabled Persons Tax Credit } & No & $40(17.2)$ & $76(16.2)$ \\
\hline & Yes & $2(0.9)$ & $3(0.6)$ \\
\hline & Missing & $191(82.0)$ & $389(83.1)$ \\
\hline \multirow[t]{3}{*}{ Carer's Allowance } & No & $30(12.9)$ & $63(13.5)$ \\
\hline & Yes & $12(5.2)$ & $16(3.4)$ \\
\hline & Missing & $191(82.0)$ & $389(83.1)$ \\
\hline \multirow[t]{3}{*}{ Jobseeker's Allowance } & No & $39(16.8)$ & $72(15.4)$ \\
\hline & Yes & $3(1.3)$ & $7(1.5)$ \\
\hline & Missing & $191(82.0)$ & $389(83.1)$ \\
\hline \multirow[t]{3}{*}{ Income Support } & No & $35(15)$ & $64(13.7)$ \\
\hline & Yes & $7(3.0)$ & $15(3.2)$ \\
\hline & Missing & $191(82.0)$ & $389(83.1)$ \\
\hline \multirow[t]{3}{*}{ Working Tax Credit } & No & $24(10.3)$ & $51(10.9)$ \\
\hline & Yes & $18(7.7)$ & $28(6.0)$ \\
\hline & Missing & $191(82.0)$ & $389(83.1)$ \\
\hline \multirow[t]{3}{*}{ Child Tax Credit } & No & $30(12.9)$ & $61(13.0)$ \\
\hline & Yes & $12(5.2)$ & $18(3.9)$ \\
\hline & Missing & $191(82.0)$ & $389(83.1)$ \\
\hline \multirow[t]{3}{*}{ Council Tax Benefit } & No & $38(16.3)$ & $68(14.5)$ \\
\hline & Yes & $4(1.7)$ & II (2.4) \\
\hline & Missing & $191(82.0)$ & $389(83.1)$ \\
\hline \multirow[t]{3}{*}{ Housing Benefit } & No & 0 & 0 \\
\hline & Yes & 0 & 0 \\
\hline & Missing & $233(100.0)$ & $468(100.0)$ \\
\hline \multirow[t]{3}{*}{ Attendance Allowance } & No & 0 & 0 \\
\hline & Yes & 0 & 0 \\
\hline & Missing & $233(100.0)$ & $468(100.0)$ \\
\hline \multirow[t]{3}{*}{ Pension Credit } & No & 0 & 0 \\
\hline & Yes & 0 & 0 \\
\hline & Missing & $233(100.0)$ & $468(100.0)$ \\
\hline \multirow[t]{3}{*}{ Other } & No & 0 & 0 \\
\hline & Yes & 0 & 0 \\
\hline & Missing & $233(100.0)$ & $468(100.0)$ \\
\hline
\end{tabular}


TABLE 69 Benefit and entitlement status - 12 months

\begin{tabular}{|c|c|c|c|}
\hline & & AM (\%) & $A M+C B A(\%)$ \\
\hline \multirow[t]{3}{*}{ Statutory Sick Pay } & No & 37 (I5.9) & $76(16.2)$ \\
\hline & Yes & 0 & $2(0.4)$ \\
\hline & Missing & $196(84.1)$ & $390(83.3)$ \\
\hline \multirow[t]{3}{*}{ Incapacity Benefit } & No & $26(11.2)$ & $60(12.8)$ \\
\hline & Yes & II (4.7) & $18(3.9)$ \\
\hline & Missing & $196(84.1)$ & $390(83.3)$ \\
\hline \multirow[t]{3}{*}{ Disability Living Allowance } & No & $25(10.7)$ & $57(12.2)$ \\
\hline & Yes & $12(5.2)$ & $21(4.5)$ \\
\hline & Missing & $196(84.1)$ & $390(83.3)$ \\
\hline \multirow[t]{3}{*}{ Severe Disablement Allowance } & No & $37(15.9)$ & $77(16.5)$ \\
\hline & Yes & 0 & I (0.2) \\
\hline & Missing & $196(84.1)$ & $390(83.3)$ \\
\hline \multirow[t]{3}{*}{ Disabled Persons Tax Credit } & No & $33(14.2)$ & $78(16.7)$ \\
\hline & Yes & $3(1.3)$ & 0 \\
\hline & Missing & $197(84.6)$ & $390(83.3)$ \\
\hline \multirow[t]{3}{*}{ Carer's Allowance } & No & $31(13.3)$ & $67(14.3)$ \\
\hline & Yes & $6(2.6)$ & II (2.4) \\
\hline & Missing & $196(84.1)$ & $390(83.3)$ \\
\hline \multirow[t]{3}{*}{ Jobseeker's Allowance } & No & $32(13.7)$ & $72(15.4)$ \\
\hline & Yes & $5(2.2)$ & $5(I . I)$ \\
\hline & Missing & $196(84.1)$ & 391 (83.6) \\
\hline \multirow[t]{3}{*}{ Income Support } & No & $32(13.7)$ & $67(14.3)$ \\
\hline & Yes & $5(2.2)$ & II (2.4) \\
\hline & Missing & $196(84.1)$ & $390(83.3)$ \\
\hline \multirow[t]{3}{*}{ Working Tax Credit } & No & $23(9.9)$ & $47(10.0)$ \\
\hline & Yes & $14(6.0)$ & $31(6.6)$ \\
\hline & Missing & $196(84.1)$ & $390(83.3)$ \\
\hline \multirow[t]{3}{*}{ Child Tax Credit } & No & $30(12.9)$ & $56(12.0)$ \\
\hline & Yes & $7(3.0)$ & $22(4.7)$ \\
\hline & Missing & $196(84.1)$ & $390(83.3)$ \\
\hline \multirow[t]{3}{*}{ Council Tax Benefit } & No & $32(13.7)$ & $66(14.1)$ \\
\hline & Yes & $5(2.2)$ & $12(2.6)$ \\
\hline & Missing & $196(84.1)$ & $390(83.3)$ \\
\hline
\end{tabular}





\section{Appendix 14}

\section{Qualitative interview schedule}

\section{3/4/06 Interview Schedule for BeST version 7}

Hi my name is ... and I work at the Medical School at the University of Warwick doing research into back pain.

This study is looking at people with back pain which has lasted for more than 6 weeks. We are interested in their experience over a 12-month period.

Have you had an opportunity to read the information sheet? Do you have any questions or issues you wish me to clarify?

Would you mind if I recorded this interview?

Anything you say will remain entirely confidential to the research team and if used in any reports will be anonymised.

At the end of the interview we will discuss whether there is anything you would prefer to remove from the interview. I'll be jotting a few things down as we go along.

1. We'll be talking about your back a bit later but I'd like you to tell me a bit about yourself.

a. First your work or daily activities?

b. Your family

c. Your social activities

- Is there anyone at home normally/ now?

- What do you do in a normal day?

- Are there activities you particularly enjoy?

- Have you been in/are you in paid employment?

- What social activities do you participate in?

- What voluntary work/caring do you undertake?

- Are there things you have not mentioned that are particularly important in your life?

2. Can you tell me about your back? Perhaps you could start right from the beginning?

- e.g. Over time? What do you do? What do you think? What happens?

- Attribution

- Beliefs re: progression (past/present)

- Coping strategies (passive/active)

- Controllability (internal/external)

- Advice or explanations from anyone.

3a. Can you tell me what kind of things affect your back?

3b. How well do you cope with your back?

3c. How do you manage life with your back?

- Anything make it better or worse?

- Benefits

- Barriers/obstacles

- Reflect on intervention received.

4. You have talked about Are there any other people you can think of with whom you've talked about your back?

- Family/friends

- HCP (formal/informal consultations)

- Investigations

- Care pathways

- Explanations given.

5a. If you think back to the practitioners you've seen about your back, are there any that you really remember and why?

5b. How did you feel? What did you think of ...?

- Was the patient listened to?

- Given adequate time in appointments (not rushed)?

- Feel respected in their decisions?

- Received what they perceive to be adequate and appropriate information?

- Shared decision-making and control?

6a. What have you received to help your back?

6b. Which treatment did you have on this trial? Could you tell me what you thought of that? Any views? What was most/least helpful? Are there any changes you would make?

- Expectations

- Psychological component

- Experience before. 
7. Were you given any written material to do with you back?

- The Back Book/other

- Read it?

- What information retained?

- Critique (good/bad)

- Influenced behaviour?

8. We're going to draw a time line about your back.

- Timeline with events and treatments, get patient to suggest anything in the area representing the last 4 months

- What would you say is the greatest change in your chart? (highlight)

9. Can you read this and pick A or B? (show laminated sheet)

- What would you like from a treatment of your back?

A. I would like immediate relief, which might not last.

B. I would like to gradually improve over 12 months, and stay that way.

10. What would you do if you did not have back pain?

\section{For cognitive behavioural group only}

We may have already covered some of these questions but could I check with you about:

1. What do you think about physical activity for your back?

- Past/present.

2. How do you decide when to go back to work/ normal activities?

- Barriers

- Beliefs.
3. How do you feel about the whole process of back skills training?

- Initial assessment

- Useful/not.

4. How did you feel about the logbooks and goal setting?

- Content/format

- Useful/not

- Good bits/bits to change.

5. How did you feel about being in a group?

- Dynamics

- Interaction

- Anecdotes.

6. Any other comments about the sessions?

- Heat/light

- Facilities

- General comments.

\section{Closing the interview}

Do you have any further comments you would like to make?

What you have said in this interview will be kept strictly confidential.

Do you have any concerns about the interview being transcribed for use in the research? (anonymised)

Any future queries regarding the trial, you have contact details on your information sheet.

Thank you for taking part in this interview study. 


\section{Appendix 15}

\section{Generalised NHS scenario as a secondary analysis}

\section{The cost of cognitive behavioural therapy in the NHS setting}

We modelled two versions of the CBA delivery. The cost of CBA included the cost of services initiated because of the health-care delivery arrangements in the trial. For example, the assumption that staff could be seconded from other institutions and travel costs were then paid was a specific consequence of the experimental nature of the CBA programme.

An alternative cost-utility scenario was developed based on the potential resource consumption for the CBA programme delivered within NHS structures. As therapists with salaried contracts are almost entirely based at one site and as travel to venues was a sizeable proportion of non-contact time, an alternative CBA resource consumption basket was profiled assuming that the delivery of CBA occurs within the existing NHS structures.

This resource consumption profile was constructed using information from both experts and published sources ${ }^{136}$ and represents an estimate of the true cost of the intervention if implemented into the NHS.

We calculated the cost of CBA as if it were delivered by the NHS incorporating the following assumptions:

- We assumed the programme delivery used sitebased staff and therefore there were no travel or mileage costs.

- We then recomputed the contact to non-contact ratio excluding total travel time.

- We assumed supervision is less intensive in NHS settings than it is in a trial; therefore reduced the supervision time to 30 minutes per CBA cycle.

- Finally, we assumed that initial training would require a refresher every 3 years, and we assumed that the cost of refreshers would be equal to that of the original training. Therefore, we assigned the cost of training in proportion to the number of cycles provided by a therapist over a period of 3 years.

\section{The cost of the $A M+C B A$ programme in the NHS setting}

Subtracting travel time from the total worked time for each cycle in the trial, we calculated that the total working time to deliver one CBA cycle in the NHS would be 15.35 hours, or 2.62 hours per session. Therefore each therapist would be able to deliver about 2.28 cycles per week, or 94.40 groups per year, based on the average number of days worked per year. The cost of CBA under the alternative NHS scenario considered was $£ 605.90$ per cycle, or $£ 126.46$ per participant.

\section{Cost-effectiveness of CBA in the NHS routine delivery setting}

In this section the cost-utility analysis is calculated under the assumption that CBAs were delivered in routine care within NHS existing structures. We computed incremental costs and QALYs and incremental cost-utility ratios for the overall population and by back pain severity using the RMQ-score subgroups.

\section{NHS perspective}

A cognitive behavioural approach was costeffective from the NHS perspective, yielding 0.099 incremental QALYs at an incremental cost of $£ 95.64$, therefore with an incremental cost-utility ratio of £966. The cost-effectiveness of CBA for individuals with severe back pain $(\mathrm{RMQ}>4)$ was similar $(\mathrm{ICUR}=£ 856)$; however, $\mathrm{CBA}$ remained dominated for the low-severity group (RMQ $\geq$ 4) (Table 70). The cost-effectiveness acceptability curves showed that for customary cost-effectiveness thresholds, CBA was cost-effective with probability nearing 1 in the whole population, but not in the low-severity population (Figure 25).

\section{General health-care perspective - NHS generalisable costs}

The cost-effectiveness of CBA from the health-care perspective using NHS generalisable costs was not 
TABLE 70 Incremental costs and QALYs, AM+CBA compared with AM, assuming NHS routine delivery, overall study population and by Roland Morris Questionnaire scores, NHS perspective

\begin{tabular}{|c|c|c|c|c|c|c|c|}
\hline & \multicolumn{2}{|l|}{ AM } & \multicolumn{2}{|c|}{$A M+C B A$} & \multirow{2}{*}{$\begin{array}{l}\text { Incremental } \\
\text { cost }(E)\end{array}$} & \multirow{2}{*}{$\begin{array}{l}\text { Incremental } \\
\text { QALYs }\end{array}$} & \multirow[b]{2}{*}{ ICER (E) } \\
\hline & Cost (t) & QALY & Cost (t) & QALY & & & \\
\hline $\begin{array}{l}\text { Overall study } \\
\text { population }\end{array}$ & 224.98 & 0.604 & 320.62 & 0.703 & 95.64 & 0.099 & 966 \\
\hline $\begin{array}{l}\text { RMQ score at } \\
\text { baseline }<4\end{array}$ & 237.58 & 0.584 & 342.90 & 0.707 & 105.32 & 0.123 & 856 \\
\hline $\begin{array}{l}\text { RMQ score at } \\
\text { baseline } \geq 4\end{array}$ & 153.59 & 0.729 & 214.39 & 0.721 & 60.80 & -0.008 & Dominated \\
\hline
\end{tabular}

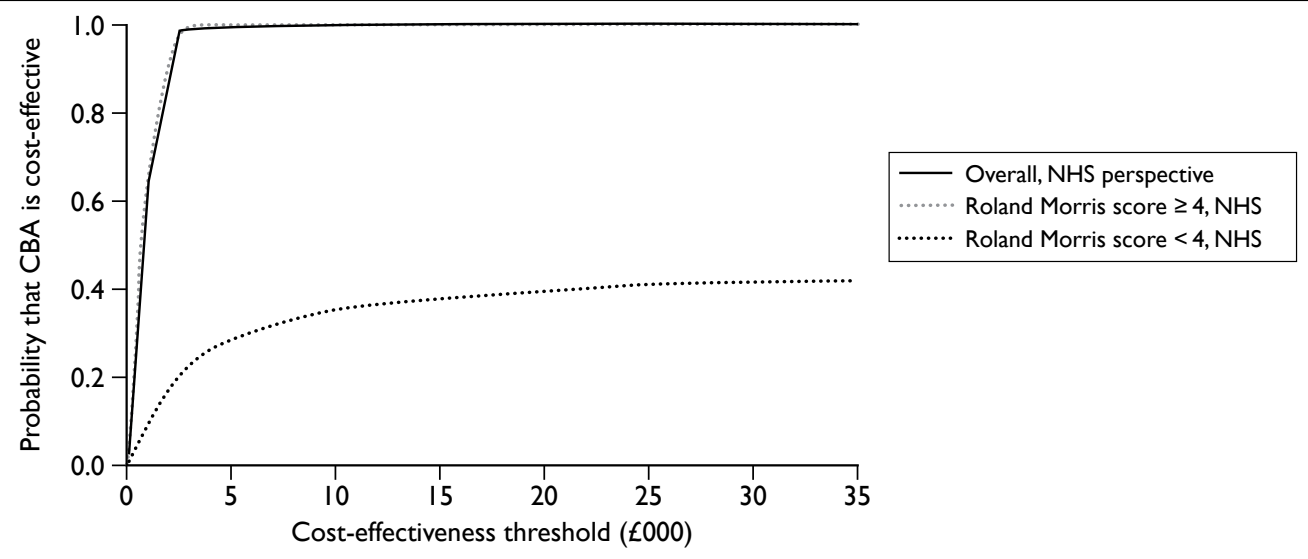

FIGURE 25 Cost-effectiveness acceptability curves, $A M+C B A$ versus $A M-N H S$ perspective, assuming NHS routine delivery, overall study population and by Roland Morris Questionnaire scores.

very different, at an incremental cost-utility ratio of $£ 2307$, yielding 0.099 incremental QALYs at an incremental cost of $£ 228.40$. The cost-effectiveness of CBA remained unfavourable for individuals with low-severity back pain (Table 71) and again, the cost-effectiveness acceptability curves showed that for customary cost-effectiveness thresholds, CBA was cost-effective with high probability in the whole population, but not in the low-severity population (Figure 26).

TABLE 7 I Incremental costs and QALYs, AM+CBA compared with AM, assuming NHS routine delivery, overall study population and by Roland Morris Questionnaire scores, health-care perspective

\begin{tabular}{|c|c|c|c|c|c|c|c|}
\hline & \multicolumn{2}{|l|}{$A M$} & \multicolumn{2}{|c|}{$A M+C B A$} & \multirow{2}{*}{$\begin{array}{l}\text { Incremental } \\
\text { cost }(t)\end{array}$} & \multirow{2}{*}{$\begin{array}{l}\text { Incremental } \\
\text { QALYs }\end{array}$} & \multirow[b]{2}{*}{$\operatorname{ICER}(t)$} \\
\hline & Cost $(t)$ & QALY & Cost $(E)$ & QALY & & & \\
\hline $\begin{array}{l}\text { Overall study } \\
\text { population }\end{array}$ & 487.34 & 0.604 & 715.74 & 0.703 & 228.40 & 0.099 & 2307 \\
\hline $\begin{array}{l}\text { RMQ score at } \\
\text { baseline }<4\end{array}$ & 498.96 & 0.580 & 779.02 & 0.707 & 280.10 & 0.123 & 2277 \\
\hline $\begin{array}{l}\text { RMQ score at } \\
\text { baseline } \geq 4\end{array}$ & 404.03 & 0.729 & 418.12 & 0.721 & 14.09 & -0.008 & Dominatec \\
\hline
\end{tabular}




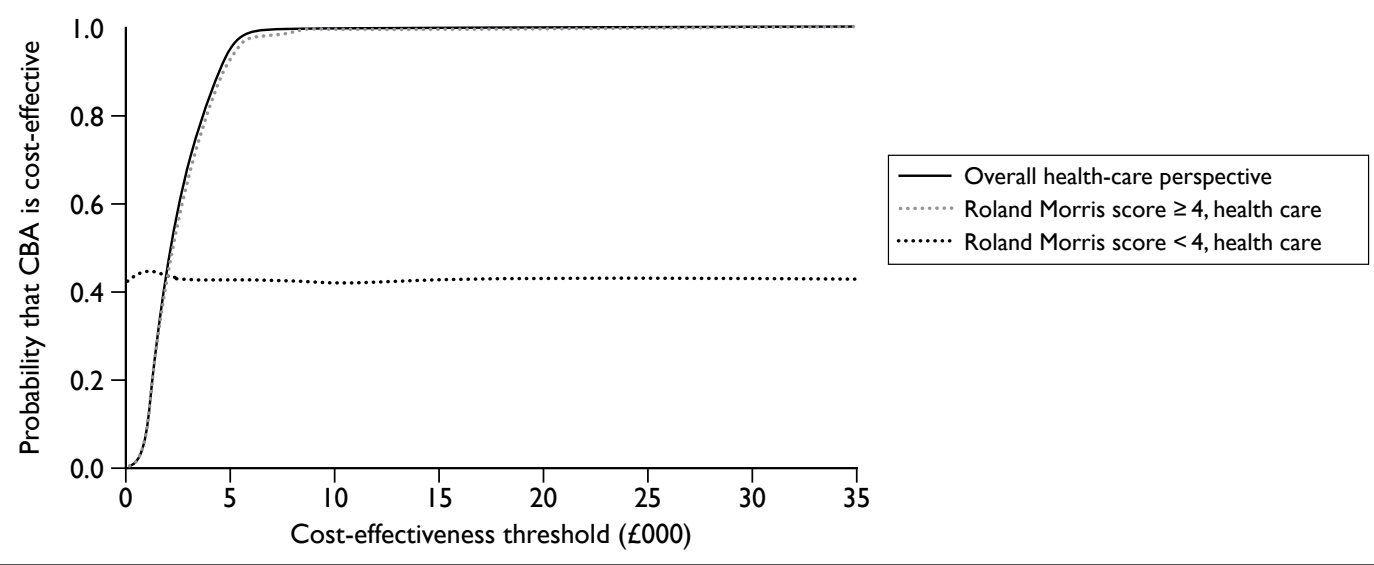

FIGURE 26 Cost-effectiveness acceptability curves, $A M+C B A$ versus $A M$, assuming NHS routine delivery, overall study population and by Roland Morris Questionnaire scores, health-care perspective. 



\section{Health Technology Assessment reports published to date}

\section{Volume I, 1997}

No. 1

Home parenteral nutrition: a systematic review.

By Richards DM, Deeks JJ, Sheldon TA, Shaffer JL.

No. 2

Diagnosis, management and screening of early localised prostate cancer.

A review by Selley S, Donovan J, Faulkner A, Coast J, Gillatt D.

No. 3

The diagnosis, management, treatment and costs of prostate cancer in England and Wales.

A review by Chamberlain J, Melia J, Moss S, Brown J.

No. 4

Screening for fragile $\mathrm{X}$ syndrome. A review by Murray J, Cuckle $\mathrm{H}$, Taylor G, Hewison J.

No. 5

A review of near patient testing in primary care.

By Hobbs FDR, Delaney BC, Fitzmaurice DA, Wilson S, Hyde CJ, Thorpe GH, et al.

No. 6

Systematic review of outpatient services for chronic pain control.

By McQuay HJ, Moore RA, Eccleston C, Morley S, de C Williams AC.

No. 7

Neonatal screening for inborn errors of metabolism: cost, yield and outcome.

A review by Pollitt RJ, Green A,

McCabe CJ, Booth A, Cooper NJ,

Leonard JV, et al.

No. 8

Preschool vision screening.

A review by Snowdon SK,

Stewart-Brown SL.

No. 9

Implications of socio-cultural contexts for the ethics of clinical trials.

A review by Ashcroft RE, Chadwick DW, Clark SRL, Edwards RHT, Frith L, Hutton JL.

No. 10

A critical review of the role of neonatal hearing screening in the detection of congenital hearing impairment.

By Davis A, Bamford J, Wilson I,

Ramkalawan T, Forshaw M, Wright S.
No. 11

Newborn screening for inborn errors of metabolism: a systematic review. By Seymour CA, Thomason MJ, Chalmers RA, Addison GM, Bain MD, Cockburn F, et al.

No. 12

Routine preoperative testing: a

systematic review of the evidence. By Munro J, Booth A, Nicholl J.

No. 13

Systematic review of the effectiveness of laxatives in the elderly.

By Petticrew M, Watt I, Sheldon T.

\section{No. 14}

When and how to assess fast-changing technologies: a comparative study of medical applications of four generic technologies.

A review by Mowatt G, Bower DJ, Brebner JA, Cairns JA, Grant AM, McKee L.

\section{Volume 2, 1998}

No. 1

Antenatal screening for Down's syndrome.

A review by Wald NJ, Kennard A, Hackshaw A, McGuire A.

No. 2

Screening for ovarian cancer: a systematic review.

By Bell R, Petticrew M, Luengo S, Sheldon TA.

No. 3

Consensus development methods, and their use in clinical guideline development.

A review by Murphy MK, Black NA, Lamping DL, McKee CM, Sanderson CFB, Askham J, et al.

No. 4

A cost-utility analysis of interferon beta for multiple sclerosis.

By Parkin D, McNamee P, Jacoby A, Miller P, Thomas S, Bates D.

No. 5

Effectiveness and efficiency of methods of dialysis therapy for end-stage renal disease: systematic reviews.

By MacLeod A, Grant A, Donaldson

C, Khan I, Campbell M, Daly C, et al.
No. 6

Effectiveness of hip prostheses in primary total hip replacement: a critical review of evidence and an economic model.

By Faulkner A, Kennedy LG, Baxter K, Donovan J, Wilkinson M, Bevan G.

No. 7

Antimicrobial prophylaxis in colorectal surgery: a systematic review of randomised controlled trials.

By Song F, Glenny AM.

No. 8

Bone marrow and peripheral blood stem cell transplantation for malignancy.

A review by Johnson PWM, Simnett SJ, Sweetenham JW, Morgan GJ Stewart LA.

No. 9

Screening for speech and language delay: a systematic review of the literature.

By Law J, Boyle J, Harris F, Harkness A, Nye C.

No. 10

Resource allocation for chronic stable angina: a systematic review of effectiveness, costs and cost-effectiveness of alternative interventions.

By Sculpher MJ, Petticrew M, Kelland JL, Elliott RA, Holdright DR, Buxton MJ.

No. 11

Detection, adherence and control of hypertension for the prevention of stroke: a systematic review.

By Ebrahim S.

No. 12

Postoperative analgesia and vomiting, with special reference to day-case surgery: a systematic review.

By McQuay HJ, Moore RA.

No. 13

Choosing between randomised and nonrandomised studies: a systematic review.

By Britton A, McKee M, Black N, McPherson K, Sanderson C, Bain C.

No. 14

Evaluating patient-based outcome measures for use in clinical trials.

A review by Fitzpatrick R, Davey C, Buxton MJ, Jones DR. 


\section{No. 15}

Ethical issues in the design and conduct of randomised controlled trials.

A review by Edwards SJL, Lilford RJ, Braunholtz DA, Jackson JC, Hewison J, Thornton J.

\section{No. 16}

Qualitative research methods in health technology assessment: a review of the literature.

By Murphy E, Dingwall R, Greatbatch D, Parker S, Watson P.

\section{No. 17}

The costs and benefits of paramedic skills in pre-hospital trauma care.

By Nicholl J, Hughes S, Dixon S, Turner J, Yates D.

\section{No. 18}

Systematic review of endoscopic ultrasound in gastro-oesophageal cancer.

By Harris KM, Kelly S, Berry E, Hutton J, Roderick P, Cullingworth J, et al.

\section{No. 19}

Systematic reviews of trials and other studies.

By Sutton AJ, Abrams KR, Jones DR, Sheldon TA, Song F.

\section{No. 20}

Primary total hip replacement surgery: a systematic review of outcomes and modelling of cost-effectiveness associated with different prostheses.

A review by Fitzpatrick R, Shortall E, Sculpher M, Murray D, Morris R, Lodge $\mathrm{M}$, et al.

\section{Volume 3, 1999}

\section{No. 1}

Informed decision making: an annotated bibliography and systematic review.

By Bekker H, Thornton JG, Airey CM, Connelly JB, Hewison J, Robinson MB, et al.

No. 2

Handling uncertainty when performing economic evaluation of healthcare interventions.

A review by Briggs AH, Gray AM.

No. 3

The role of expectancies in the placebo effect and their use in the delivery of health care: a systematic review.

By Crow R, Gage H, Hampson S, Hart J, Kimber A, Thomas H.

\section{No. 4}

A randomised controlled trial of different approaches to universal antenatal HIV testing: uptake and acceptability. Annex: Antenatal HIV testing - assessment of a routine voluntary approach.

By Simpson WM, Johnstone FD, Boyd FM, Goldberg DJ, Hart GJ, Gormley SM, et al.

\section{No. 5}

Methods for evaluating area-wide and organisation-based interventions in health and health care: a systematic review.

By Ukoumunne OC, Gulliford MC, Chinn S, Sterne JAC, Burney PGJ

\section{No. 6}

Assessing the costs of healthcare technologies in clinical trials.

A review by Johnston K, Buxton MJ, Jones DR, Fitzpatrick R.

No. 7

Cooperatives and their primary care emergency centres: organisation and impact.

By Hallam L, Henthorne K.

No. 8

Screening for cystic fibrosis A review by Murray J, Cuckle $\mathrm{H}$, Taylor G, Littlewood J, Hewison J.

\section{No. 9}

A review of the use of health status measures in economic evaluation.

By Brazier J, Deverill M, Green C, Harper R, Booth A.

No. 10

Methods for the analysis of qualityof-life and survival data in health technology assessment.

A review by Billingham LJ, Abrams KR, Jones DR.

\section{No. 11}

Antenatal and neonata haemoglobinopathy screening in the UK: review and economic analysis. By Zeuner D, Ades AE, Karnon J, Brown J, Dezateux C, Anionwu EN.

\section{No. 12}

Assessing the quality of reports of randomised trials: implications for the conduct of meta-analyses.

A review by Moher D, Cook DJ, Jadad AR, Tugwell P, Moher M, Jones A, et al.

No. 13

'Early warning systems' for identifying new healthcare technologies.

By Robert G, Stevens A, Gabbay J.

No. 14

A systematic review of the role of human papillomavirus testing within a cervical screening programme.

By Cuzick J, Sasieni P, Davies P, Adams J, Normand C, Frater A, et al.
No. 15

Near patient testing in diabetes clinics: appraising the costs and outcomes.

By Grieve R, Beech R, Vincent J, Mazurkiewicz J.

No. 16

Positron emission tomography: establishing priorities for health technology assessment.

A review by Robert G, Milne $\mathrm{R}$.

\section{No. 17 (Pt 1)}

The debridement of chronic wounds: a systematic review.

By Bradley M, Cullum N, Sheldon T.

\section{No. 17 (Pt 2)}

Systematic reviews of wound care management: (2) Dressings and topical agents used in the healing of chronic wounds.

By Bradley M, Cullum N, Nelson EA, Petticrew M, Sheldon T, Torgerson D.

\section{No. 18}

A systematic literature review of spiral and electron beam computed tomography: with particular reference to clinical applications in hepatic lesions, pulmonary embolus and coronary artery disease.

By Berry E, Kelly S, Hutton J, Harris KM, Roderick P, Boyce JC, et al.

\section{No. 19}

What role for statins? A review and economic model.

By Ebrahim S, Davey Smith G, McCabe C, Payne N, Pickin M, Sheldon TA, et al.

No. 20

Factors that limit the quality, number and progress of randomised controlled trials.

A review by Prescott RJ, Counsell CE, Gillespie WJ, Grant AM, Russell IT,

Kiauka S, et al.

No. 21

Antimicrobial prophylaxis in total hip replacement: a systematic review.

By Glenny AM, Song F.

No. 22

Health promoting schools and health promotion in schools: two systematic reviews.

By Lister-Sharp D, Chapman S, Stewart-Brown S, Sowden A.

No. 23

Economic evaluation of a primary care-based education programme for patients with osteoarthritis of the knee.

A review by Lord J, Victor C, Littlejohns P, Ross FM, Axford JS 


\section{Volume 4, 2000}

No. 1

The estimation of marginal time preference in a UK-wide sample (TEMPUS) project.

A review by Cairns JA, van der Pol MM.

\section{No. 2}

Geriatric rehabilitation following fractures in older people: a systematic review.

By Cameron I, Crotty M, Currie C, Finnegan T, Gillespie L, Gillespie W, et al.

\section{No. 3}

Screening for sickle cell disease and thalassaemia: a systematic review with supplementary research.

By Davies SC, Cronin E, Gill M, Greengross P, Hickman M, Normand C.

\section{No. 4}

Community provision of hearing aids and related audiology services.

A review by Reeves DJ, Alborz A, Hickson FS, Bamford JM.

No. 5

False-negative results in screening programmes: systematic review of impact and implications.

By Petticrew MP, Sowden AJ, Lister-Sharp D, Wright K.

\section{No. 6}

Costs and benefits of community postnatal support workers: a randomised controlled trial.

By Morrell CJ, Spiby H, Stewart P, Walters S, Morgan A.

\section{No. 7}

Implantable contraceptives (subdermal implants and hormonally impregnated intrauterine systems) versus other forms of reversible contraceptives: two systematic reviews to assess relative effectiveness, acceptability, tolerability and cost-effectiveness.

By French RS, Cowan FM, Mansour DJA, Morris S, Procter T, Hughes D, et al.

No. 8

An introduction to statistical methods for health technology assessment.

A review by White SJ, Ashby D, Brown PJ.

No. 9

Disease-modifying drugs for multiple sclerosis: a rapid and systematic review.

By Clegg A, Bryant J, Milne R.

\section{No. 10}

Publication and related biases.

A review by Song F, Eastwood AJ, Gilbody S, Duley L, Sutton AJ.

\section{No. 11}

Cost and outcome implications of the organisation of vascular services.

By Michaels J, Brazier J, Palfreyman S, Shackley P, Slack R.

No. 12

Monitoring blood glucose control in diabetes mellitus: a systematic review.

By Coster S, Gulliford MC, Seed PT, Powrie JK, Swaminathan R.

\section{No. 13}

The effectiveness of domiciliary health visiting: a systematic review of international studies and a selective review of the British literature.

By Elkan R, Kendrick D, Hewitt M, Robinson JJA, Tolley K, Blair M, et al.

No. 14

The determinants of screening uptake and interventions for increasing uptake: a systematic review.

By Jepson R, Clegg A, Forbes C, Lewis R, Sowden A, Kleijnen J.

\section{No. 15}

The effectiveness and cost-effectiveness of prophylactic removal of wisdom teeth.

A rapid review by Song F, O'Meara $S$, Wilson P, Golder S, Kleijnen J.

\section{No. 16}

Ultrasound screening in pregnancy: a systematic review of the clinical effectiveness, cost-effectiveness and women's views.

By Bricker L, Garcia J, Henderson J, Mugford M, Neilson J, Roberts T, et al.

\section{No. 17}

A rapid and systematic review of the effectiveness and cost-effectiveness of the taxanes used in the treatment of advanced breast and ovarian cancer.

By Lister-Sharp D, McDonagh MS, Khan KS, Kleijnen J.

No. 18

Liquid-based cytology in cervical screening: a rapid and systematic review.

By Payne N, Chilcott J, McGoogan E.

No. 19

Randomised controlled trial of nondirective counselling, cognitivebehaviour therapy and usual general practitioner care in the management of depression as well as mixed anxiety and depression in primary care.

By King M, Sibbald B, Ward E, Bower P, Lloyd M, Gabbay M, et al.

\section{No. 20}

Routine referral for radiography of patients presenting with low back pain: is patients' outcome influenced by GPs' referral for plain radiography?

By Kerry S, Hilton S, Patel S, Dundas D, Rink E, Lord J.
No. 21

Systematic reviews of wound care management: (3) antimicrobial agents for chronic wounds; (4) diabetic foot ulceration.

By O'Meara S, Cullum N, Majid M, Sheldon T.

No. 22

Using routine data to complement and enhance the results of randomised controlled trials.

By Lewsey JD, Leyland AH, Murray GD, Boddy FA.

\section{No. 23}

Coronary artery stents in the treatment of ischaemic heart disease: a rapid and systematic review.

By Meads C, Cummins C, Jolly K, Stevens A, Burls A, Hyde C.

\section{No. 24}

Outcome measures for adult critical care: a systematic review.

By Hayes JA, Black NA, Jenkinson C, Young JD, Rowan KM, Daly K, et al.

No. 25

A systematic review to evaluate the effectiveness of interventions to promote the initiation of breastfeeding.

By Fairbank L, O'Meara S,

Renfrew MJ, Woolridge M, Sowden AJ, Lister-Sharp D.

\section{No. 26}

Implantable cardioverter defibrillators: arrhythmias. A rapid and systematic review.

By Parkes J, Bryant J, Milne R.

No. 27

Treatments for fatigue in multiple sclerosis: a rapid and systematic review.

By Brañas P, Jordan R, Fry-Smith A, Burls A, Hyde C.

\section{No. 28}

Early asthma prophylaxis, natural history, skeletal development and economy (EASE): a pilot randomised controlled trial.

By Baxter-Jones ADG, Helms PJ, Russell G, Grant A, Ross S, Cairns JA, et al.

No. 29

Screening for hypercholesterolaemia versus case finding for familial hypercholesterolaemia: a systematic review and cost-effectiveness analysis.

By Marks D, Wonderling D, Thorogood M, Lambert H, Humphries SE, Neil HAW.

\section{No. 30}

A rapid and systematic review of the clinical effectiveness and costeffectiveness of glycoprotein IIb/ IIIa antagonists in the medical management of unstable angina.

By McDonagh MS, Bachmann LM Golder S, Kleijnen J, ter Riet G. 


\section{No. 31}

A randomised controlled trial of prehospital intravenous fluid replacement therapy in serious trauma.

By Turner J, Nicholl J, Webber L, Cox H, Dixon S, Yates D.

\section{No. 32}

Intrathecal pumps for giving opioids in chronic pain: a systematic review. By Williams JE, Louw G, Towlerton G.

\section{No. 33}

Combination therapy (interferon alfa and ribavirin) in the treatment of chronic hepatitis $\mathrm{C}$ : a rapid and systematic review.

By Shepherd J, Waugh N, Hewitson P.

\section{No. 34}

A systematic review of comparisons of effect sizes derived from randomised and non-randomised studies.

By MacLehose RR, Reeves BC, Harvey IM, Sheldon TA, Russell IT, Black AMS.

\section{No. 35}

Intravascular ultrasound-guided interventions in coronary artery disease: a systematic literature review, with decision-analytic modelling, of outcomes and cost-effectiveness.

By Berry E, Kelly S, Hutton J, Lindsay HSJ, Blaxill JM, Evans JA, et al.

\section{No. 36}

A randomised controlled trial to evaluate the effectiveness and costeffectiveness of counselling patients with chronic depression.

By Simpson S, Corney R, Fitzgerald P, Beecham J.

\section{No. 37}

Systematic review of treatments for atopic eczema.

By Hoare C, Li Wan Po A, Williams $\mathrm{H}$.

\section{No. 38}

Bayesian methods in health technology assessment: a review.

By Spiegelhalter DJ, Myles JP, Jones DR, Abrams KR.

\section{No. 39}

The management of dyspepsia: a systematic review.

By Delaney B, Moayyedi P, Deeks J, Innes M, Soo S, Barton P, et al.

\section{No. 40}

A systematic review of treatments for severe psoriasis.

By Griffiths CEM, Clark CM,

\section{Volume 5, 200I}

\section{No. 1}

Clinical and cost-effectiveness of donepezil, rivastigmine and galantamine for Alzheimer's disease: a rapid and systematic review.

By Clegg A, Bryant J, Nicholson T, McIntyre L, De Broe S, Gerard K, et al.

\section{No. 2}

The clinical effectiveness and costeffectiveness of riluzole for motor neurone disease: a rapid and systematic review.

By Stewart A, Sandercock J, Bryan S, Hyde C, Barton PM, Fry-Smith A, et al.

\section{No. 3}

Equity and the economic evaluation of healthcare.

By Sassi F, Archard L, Le Grand J.

No. 4

Quality-of-life measures in chronic diseases of childhood.

By Eiser C, Morse R.

\section{No. 5}

Eliciting public preferences for healthcare: a systematic review of techniques.

By Ryan M, Scott DA, Reeves C, Bate A, van Teijlingen ER, Russell EM, et al.

\section{No. 6}

General health status measures for people with cognitive impairment: learning disability and acquired brain injury.

By Riemsma RP, Forbes CA, Glanville JM, Eastwood AJ, Kleijnen J.

\section{No. 7}

An assessment of screening strategies for fragile X syndrome in the UK.

By Pembrey ME, Barnicoat AJ, Carmichael B, Bobrow M, Turner G.

\section{No. 8}

Issues in methodological research: perspectives from researchers and commissioners.

By Lilford RJ, Richardson A, Stevens A, Fitzpatrick R, Edwards S, Rock F, et al.

\section{No. 9}

Systematic reviews of wound care management: (5) beds; (6) compression; (7) laser therapy, therapeutic ultrasound, electrotherapy and electromagnetic therapy.

By Cullum N, Nelson EA, Flemming K, Sheldon T.

\section{No. 10}

Effects of educational and psychosocial interventions for adolescents with diabetes mellitus: a systematic review.

By Hampson SE, Skinner TC, Hart J, Storey L, Gage H, Foxcroft D, et al.
No. 11

Effectiveness of autologous chondrocyte transplantation for hyaline cartilage defects in knees: a rapid and systematic review.

By Jobanputra P, Parry D, Fry-Smith A, Burls A.

\section{No. 12}

Statistical assessment of the learning curves of health technologies.

By Ramsay CR, Grant AM, Wallace SA, Garthwaite PH, Monk AF, Russell IT.

\section{No. 13}

The effectiveness and cost-effectiveness of temozolomide for the treatment of recurrent malignant glioma: a rapid and systematic review.

By Dinnes J, Cave C, Huang S, Major K, Milne R.

\section{No. 14}

A rapid and systematic review of the clinical effectiveness and costeffectiveness of debriding agents in treating surgical wounds healing by secondary intention.

By Lewis R, Whiting $\mathrm{P}$, ter Riet $\mathrm{G}$, O'Meara S, Glanville J.

\section{No. 15}

Home treatment for mental health problems: a systematic review.

By Burns T, Knapp M, Catty J, Healey A, Henderson J, Watt $\mathrm{H}$, et al.

No. 16

How to develop cost-conscious guidelines.

By Eccles M, Mason J.

No. 17

The role of specialist nurses in multiple sclerosis: a rapid and systematic review.

By De Broe S, Christopher F, Waugh N.

\section{No. 18}

A rapid and systematic review of the clinical effectiveness and cost-effectiveness of orlistat in the management of obesity.

By O'Meara S, Riemsma R, Shirran L, Mather L, ter Riet G.

No. 19

The clinical effectiveness and costeffectiveness of pioglitazone for type 2 diabetes mellitus: a rapid and systematic review.

By Chilcott J, Wight J, Lloyd Jones M, Tappenden P.

No. 20

Extended scope of nursing practice: a multicentre randomised controlled trial of appropriately trained nurses and preregistration house officers in preoperative assessment in elective general surgery.

By Kinley H, Czoski-Murray C, George S, McCabe C, Primrose J, Reilly C, et al. 
No. 21

Systematic reviews of the effectiveness of day care for people with severe mental disorders: (1) Acute day hospital versus admission; (2) Vocational rehabilitation; (3) Day hospital versus outpatient care.

By Marshall M, Crowther R, Almaraz-Serrano A, Creed F, Sledge W, Kluiter $\mathrm{H}$, et al.

No. 22

The measurement and monitoring of surgical adverse events.

By Bruce J, Russell EM, Mollison J, Krukowski ZH.

\section{No. 23}

Action research: a systematic review and guidance for assessment.

By Waterman H, Tillen D, Dickson R, de Koning $\mathrm{K}$

\section{No. 24}

A rapid and systematic review of the clinical effectiveness and costeffectiveness of gemcitabine for the treatment of pancreatic cancer.

By Ward S, Morris E, Bansback N, Calvert N, Crellin A, Forman D, et al.

\section{No. 25}

A rapid and systematic review of the evidence for the clinical effectiveness and cost-effectiveness of irinotecan, oxaliplatin and raltitrexed for the treatment of advanced colorectal cancer.

By Lloyd Jones M, Hummel S, Bansback N, Orr B, Seymour M.

\section{No. 26}

Comparison of the effectiveness of inhaler devices in asthma and chronic obstructive airways disease: a systematic review of the literature.

By Brocklebank D, Ram F, Wright J, Barry P, Cates C, Davies L, et al.

\section{No. 27}

The cost-effectiveness of magnetic resonance imaging for investigation of the knee joint

By Bryan S, Weatherburn G, Bungay H, Hatrick C, Salas C, Parry D, et al.

\section{No. 28}

A rapid and systematic review of the clinical effectiveness and costeffectiveness of topotecan for ovarian cancer.

By Forbes C, Shirran L, Bagnall A-M, Duffy S, ter Riet G.

\section{No. 29}

Superseded by a report published in a later volume.

\section{No. 30}

The role of radiography in primary care patients with low back pain of at least 6 weeks duration: a randomised (unblinded) controlled trial.

By Kendrick D, Fielding K, Bentley E, Miller P, Kerslake R, Pringle M.

\section{No. 31}

Design and use of questionnaires: a review of best practice applicable to surveys of health service staff and patients.

By McColl E, Jacoby A, Thomas L, Soutter J, Bamford C, Steen N, et al.

\section{No. 32}

A rapid and systematic review of the clinical effectiveness and costeffectiveness of paclitaxel, docetaxel, gemcitabine and vinorelbine in nonsmall-cell lung cancer.

By Clegg A, Scott DA, Sidhu M, Hewitson P, Waugh N.

\section{No. 33}

Subgroup analyses in randomised controlled trials: quantifying the risks of false-positives and false-negatives.

By Brookes ST, Whitley E, Peters TJ, Mulheran PA, Egger M, Davey Smith G.

\section{No. 34}

Depot antipsychotic medication in the treatment of patients with schizophrenia: (1) Meta-review; (2) Patient and nurse attitudes.

By David AS, Adams C.

No. 35

A systematic review of controlled trials of the effectiveness and costeffectiveness of brief psychological treatments for depression.

By Churchill R, Hunot V, Corney R, Knapp M, McGuire H, Tylee A, et al.

No. 36

Cost analysis of child health surveillance.

By Sanderson D, Wright D, Acton C, Duree D.

\section{Volume 6, 2002}

\section{No. 1}

A study of the methods used to select review criteria for clinical audit.

By Hearnshaw H, Harker R, Cheater F, Baker R, Grimshaw G.

\section{No. 2}

Fludarabine as second-line therapy for B cell chronic lymphocytic leukaemia: a technology assessment.

By Hyde C, Wake B, Bryan S, Barton P, Fry-Smith A, Davenport C, et al.

\section{No. 3}

Rituximab as third-line treatment for refractory or recurrent Stage III or IV follicular non-Hodgkin's lymphoma: a systematic review and economic evaluation.

By Wake B, Hyde C, Bryan S, Barton P, Song F, Fry-Smith A, et al.

\section{No. 4}

A systematic review of discharge arrangements for older people.

By Parker SG, Peet SM, McPherson A, Cannaby AM, Baker R, Wilson A, et al.

No. 5

The clinical effectiveness and costeffectiveness of inhaler devices used in the routine management of chronic asthma in older children: a systematic review and economic evaluation.

By Peters J, Stevenson M, Beverley C, Lim J, Smith S.

No. 6

The clinical effectiveness and costeffectiveness of sibutramine in the management of obesity: a technology assessment.

By O'Meara S, Riemsma R, Shirran L, Mather L, ter Riet $\mathrm{G}$.

No. 7

The cost-effectiveness of magnetic resonance angiography for carotid artery stenosis and peripheral vascular disease: a systematic review.

By Berry E, Kelly S, Westwood ME Davies LM, Gough MJ, Bamford JM, et al.

\section{No. 8}

Promoting physical activity in South Asian Muslim women through 'exercise on prescription'.

By Carroll B, Ali N, Azam N.

No. 9

Zanamivir for the treatment of influenza in adults: a systematic review and economic evaluation.

By Burls A, Clark W, Stewart T, Preston C, Bryan S, Jefferson T, et al.

\section{No. 10}

A review of the natural history and epidemiology of multiple sclerosis: implications for resource allocation and health economic models.

By Richards RG, Sampson FC, Beard SM, Tappenden P.

No. 11

Screening for gestational diabetes:

a systematic review and economic evaluation.

By Scott DA, Loveman E, McIntyre L, Waugh N.

No. 12

The clinical effectiveness and costeffectiveness of surgery for people with morbid obesity: a systematic review and economic evaluation.

By Clegg AJ, Colquitt J, Sidhu MK, Royle P, Loveman E, Walker A.

No. 13

The clinical effectiveness of trastuzumab for breast cancer: a systematic review.

By Lewis R, Bagnall A-M, Forbes C, Shirran E, Duffy S, Kleijnen J, et al. 


\section{No. 14}

The clinical effectiveness and costeffectiveness of vinorelbine for breast cancer: a systematic review and economic evaluation.

By Lewis R, Bagnall A-M, King S, Woolacott N, Forbes C, Shirran L, et al.

\section{No. 15}

A systematic review of the effectiveness and cost-effectiveness of metal-onmetal hip resurfacing arthroplasty for treatment of hip disease.

By Vale L, Wyness L, McCormack K, McKenzie L, Brazzelli M, Stearns SC.

\section{No. 16}

The clinical effectiveness and costeffectiveness of bupropion and nicotine replacement therapy for smoking cessation: a systematic review and economic evaluation.

By Woolacott NF, Jones L, Forbes CA, Mather LC, Sowden AJ, Song FJ, et al.

\section{No. 17}

A systematic review of effectiveness and economic evaluation of new drug treatments for juvenile idiopathic arthritis: etanercept.

By Cummins C, Connock M, Fry-Smith A, Burls A.

\section{No. 18}

Clinical effectiveness and costeffectiveness of growth hormone in children: a systematic review and economic evaluation.

By Bryant J, Cave C, Mihaylova B, Chase D, McIntyre L, Gerard K, et al.

\section{No. 19}

Clinical effectiveness and costeffectiveness of growth hormone in adults in relation to impact on quality of life: a systematic review and economic evaluation.

By Bryant J, Loveman E, Chase D, Mihaylova B, Cave C, Gerard K, et al.

\section{No. 20}

Clinical medication review by a pharmacist of patients on repeat prescriptions in general practice: a randomised controlled trial.

By Zermansky AG, Petty DR, Raynor DK, Lowe CJ, Freementle N, Vail A.

\section{No. 21}

The effectiveness of infliximab and etanercept for the treatment of rheumatoid arthritis: a systematic review and economic evaluation.

By Jobanputra P, Barton P, Bryan S, Burls A.

\section{No. 22}

A systematic review and economic evaluation of computerised cognitive behaviour therapy for depression and anxiety.

By Kaltenthaler E, Shackley P, Stevens K, Beverley C, Parry G, Chilcott J
No. 23

A systematic review and economic evaluation of pegylated liposomal doxorubicin hydrochloride for ovarian cancer.

By Forbes C, Wilby J, Richardson G, Sculpher M, Mather L, Riemsma R.

\section{No. 24}

A systematic review of the effectivenes of interventions based on a stages-ofchange approach to promote individual behaviour change.

By Riemsma RP, Pattenden J, Bridle C, Sowden AJ, Mather L, Watt IS, et al.

\section{No. 25}

A systematic review update of the clinical effectiveness and costeffectiveness of glycoprotein IIb/IIIa antagonists.

By Robinson M, Ginnelly L, Sculpher M, Jones L, Riemsma R, Palmer S, et al.

\section{No. 26}

A systematic review of the effectiveness, cost-effectiveness and barriers to implementation of thrombolytic and neuroprotective therapy for acute ischaemic stroke in the NHS

By Sandercock P, Berge E, Dennis M, Forbes J, Hand P, Kwan J, et al.

No. 27

A randomised controlled crossover trial of nurse practitioner versus doctor-

led outpatient care in a bronchiectasis clinic.

By Caine N, Sharples LD,

Hollingworth W, French J, Keogan M, Exley A, et al.

No. 28

Clinical effectiveness and cost consequences of selective serotonin reuptake inhibitors in the treatment of sex offenders.

By Adi Y, Ashcroft D, Browne K, Beech A, Fry-Smith A, Hyde C.

\section{No. 29}

Treatment of established osteoporosis: a systematic review and cost-utility analysis.

By Kanis JA, Brazier JE, Stevenson M, Calvert NW, Lloyd Jones M.

\section{No. 30}

Which anaesthetic agents are costeffective in day surgery? Literature review, national survey of practice and randomised controlled trial.

By Elliott RA, Payne K, Moore JK, Davies LM, Harper NJN, St Leger AS, et al.

\section{No. 31}

Screening for hepatitis $\mathrm{C}$ among injecting drug users and in genitourinary medicine clinics: systematic reviews of effectiveness, modelling study and national survey of current practice.

By Stein K, Dalziel K, Walker A McIntyre L, Jenkins B, Horne J, et al.
No. 32

The measurement of satisfaction with healthcare: implications for practice from a systematic review of the literature.

By Crow R, Gage H, Hampson S, Hart J, Kimber A, Storey L, et al.

No. 33

The effectiveness and cost-effectiveness of imatinib in chronic myeloid leukaemia: a systematic review.

By Garside R, Round A, Dalziel K, Stein K, Royle R.

No. 34

A comparative study of hypertonic saline, daily and alternate-day rhDNase in children with cystic fibrosis.

By Suri R, Wallis C, Bush A

Thompson S, Normand C, Flather M, et al.

No. 35

A systematic review of the costs and effectiveness of different models of paediatric home care.

By Parker G, Bhakta P, Lovett CA, Paisley S, Olsen R, Turner D, et al.

\section{Volume 7, 2003}

No. 1

How important are comprehensive literature searches and the assessment of trial quality in systematic reviews? Empirical study.

By Egger M, Jüni P, Bartlett C, Holenstein F, Sterne J.

No. 2

Systematic review of the effectiveness and cost-effectiveness, and economic evaluation, of home versus hospital or satellite unit haemodialysis for people with end-stage renal failure.

By Mowatt G, Vale L, Perez J, Wyness L, Fraser C, MacLeod A, et al.

No. 3

Systematic review and economic evaluation of the effectiveness of infliximab for the treatment of Crohn's disease.

By Clark W, Raftery J, Barton P, Song F, Fry-Smith A, Burls A.

No. 4

A review of the clinical effectiveness and cost-effectiveness of routine anti-D prophylaxis for pregnant women who are rhesus negative.

By Chilcott J, Lloyd Jones M, Wight J, Forman K, Wray J, Beverley C, et al.

No. 5

Systematic review and evaluation of the use of tumour markers in paediatric oncology: Ewing's sarcoma and neuroblastoma.

By Riley RD, Burchill SA, Abrams KR, Heney D, Lambert PC, Jones DR, et al. 


\section{No. 6}

The cost-effectiveness of screening for Helicobacter pylori to reduce mortality and morbidity from gastric cancer and peptic ulcer disease: a discrete-event simulation model.

By Roderick P, Davies R, Raftery J, Crabbe D, Pearce R, Bhandari P, et al.

\section{No. 7}

The clinical effectiveness and costeffectiveness of routine dental checks a systematic review and economic evaluation.

By Davenport C, Elley K, Salas C, Taylor-Weetman CL, Fry-Smith A, Bryan S, et al.

\section{No. 8}

A multicentre randomised controlled trial assessing the costs and benefits of using structured information and analysis of women's preferences in the management of menorrhagia.

By Kennedy ADM, Sculpher MJ, Coulter A, Dwyer N, Rees M, Horsley S, et al.

\section{No. 9}

Clinical effectiveness and cost-utility of photodynamic therapy for wet age-related macular degeneration: a systematic review and economic evaluation.

By Meads C, Salas C, Roberts T, Moore D, Fry-Smith A, Hyde C.

\section{No. 10}

Evaluation of molecular tests for prenatal diagnosis of chromosome abnormalities.

By Grimshaw GM, Szczepura A, Hultén M, MacDonald F, Nevin NC, Sutton $\mathrm{F}$, et al.

\section{No. 11}

First and second trimester antenatal screening for Down's syndrome: the results of the Serum, Urine and Ultrasound Screening Study (SURUSS).

By Wald NJ, Rodeck C, Hackshaw AK, Walters J, Chitty L, Mackinson AM.

\section{No. 12}

The effectiveness and cost-effectiveness of ultrasound locating devices for central venous access: a systematic review and economic evaluation.

By Calvert N, Hind D, McWilliams RG, Thomas SM, Beverley C, Davidson A.

\section{No. 13}

A systematic review of atypical antipsychotics in schizophrenia.

By Bagnall A-M, Jones L, Lewis R, Ginnelly L, Glanville J, Torgerson D, et al.

\section{No. 14}

Prostate Testing for Cancer and Treatment (ProtecT) feasibility study.

By Donovan J, Hamdy F, Neal D, Peters T, Oliver S, Brindle L, et al.

\section{No. 15}

Early thrombolysis for the treatment of acute myocardial infarction: a systematic review and economic evaluation.

By Boland A, Dundar Y, Bagust A, Haycox A, Hill R, Mujica Mota R, et al.

\section{No. 16}

Screening for fragile X syndrome: a literature review and modelling.

By Song FJ, Barton P, Sleightholme V, Yao GL, Fry-Smith A.

\section{No. 17}

Systematic review of endoscopic sinus surgery for nasal polyps.

By Dalziel K, Stein K, Round A, Garside R, Royle P.

\section{No. 18}

Towards efficient guidelines: how to monitor guideline use in primary care.

By Hutchinson A, McIntosh A, Cox S, Gilbert C.

\section{No. 19}

Effectiveness and cost-effectiveness of acute hospital-based spinal cord injuries services: systematic review.

By Bagnall A-M, Jones L, Richardson G, Duffy S, Riemsma R.

\section{No. 20}

Prioritisation of health technology assessment. The PATHS model: methods and case studies.

By Townsend J, Buxton M, Harper G.

\section{No. 21}

Systematic review of the clinical effectiveness and cost-effectiveness of tension-free vaginal tape for treatment of urinary stress incontinence.

By Cody J, Wyness L, Wallace S, Glazener C, Kilonzo M, Stearns S, et al.

\section{No. 22}

The clinical and cost-effectiveness of patient education models for diabetes: a systematic review and economic evaluation.

By Loveman E, Cave C, Green C, Royle P, Dunn N, Waugh N.

\section{No. 23}

The role of modelling in prioritising and planning clinical trials.

By Chilcott J, Brennan A, Booth A, Karnon J, Tappenden P.

\section{No. 24}

Cost-benefit evaluation of routine influenza immunisation in people 65-74 years of age.

By Allsup S, Gosney M, Haycox A, Regan M.

\section{No. 25}

The clinical and cost-effectiveness of pulsatile machine perfusion versus cold storage of kidneys for transplantation retrieved from heart-beating and nonheart-beating donors.

By Wight J, Chilcott J, Holmes M, Brewer N.

\section{No. 26}

Can randomised trials rely on existing electronic data? A feasibility study to explore the value of routine data in health technology assessment.

By Williams JG, Cheung WY,

Cohen DR, Hutchings HA, Longo MF Russell IT.

No. 27

Evaluating non-randomised

intervention studies.

By Deeks JJ, Dinnes J, D’Amico R, Sowden AJ, Sakarovitch C, Song F, et al.

\section{No. 28}

A randomised controlled trial to assess the impact of a package comprising a patient-orientated, evidence-based selfhelp guidebook and patient-centred consultations on disease management and satisfaction in inflammatory bowel disease.

By Kennedy A, Nelson E, Reeves D, Richardson G, Roberts C, Robinson A, et al.

\section{No. 29}

The effectiveness of diagnostic tests for the assessment of shoulder pain due to soft tissue disorders: a systematic review.

By Dinnes J, Loveman E, McIntyre L, Waugh N.

No. 30

The value of digital imaging in diabetic retinopathy.

By Sharp PF, Olson J, Strachan F, Hipwell J, Ludbrook A, O’Donnell M, et al.

\section{No. 31}

Lowering blood pressure to prevent myocardial infarction and stroke: a new preventive strategy.

By Law M, Wald N, Morris J.

No. 32

Clinical and cost-effectiveness of capecitabine and tegafur with uracil for the treatment of metastatic colorectal cancer: systematic review and economic evaluation.

By Ward S, Kaltenthaler E, Cowan J, Brewer N.

\section{No. 33}

Clinical and cost-effectiveness of new and emerging technologies for early localised prostate cancer: a systematic review.

By Hummel S, Paisley S, Morgan A, Currie E, Brewer N. 


\section{No. 34}

Literature searching for clinical and cost-effectiveness studies used in health technology assessment reports carried out for the National Institute for Clinical Excellence appraisal system.

By Royle P, Waugh N.

\section{No. 35}

Systematic review and economic decision modelling for the prevention and treatment of influenza A and B.

By Turner D, Wailoo A, Nicholson K, Cooper N, Sutton A, Abrams K.

\section{No. 36}

A randomised controlled trial to evaluate the clinical and costeffectiveness of Hickman line insertions in adult cancer patients by nurses.

By Boland A, Haycox A, Bagust A, Fitzsimmons L.

\section{No. 37}

Redesigning postnatal care: a randomised controlled trial of protocolbased midwifery-led care focused on individual women's physical and psychological health needs.

By MacArthur C, Winter HR, Bick DE, Lilford RJ, Lancashire RJ, Knowles $\mathrm{H}$, et al.

\section{No. 38}

Estimating implied rates of discount in healthcare decision-making.

By West RR, McNabb R, Thompson AGH, Sheldon TA, Grimley Evans J.

\section{No. 39}

Systematic review of isolation policies in the hospital management of methicillin-resistant Staphylococcus aureus: a review of the literature with epidemiological and economic modelling.

By Cooper BS, Stone SP, Kibbler CC, Cookson BD, Roberts JA, Medley GF, et al.

\section{No. 40}

Treatments for spasticity and pain in multiple sclerosis: a systematic review.

By Beard S, Hunn A, Wight J.

\section{No. 41}

The inclusion of reports of randomised trials published in languages other than English in systematic reviews.

By Moher D, Pham B, Lawson ML Klassen TP

\section{No. 42}

The impact of screening on future health-promoting behaviours and health beliefs: a systematic review.

By Bankhead CR, Brett J, Bukach C, Webster P, Stewart-Brown S, Munafo M, et al.

\section{Volume 8, 2004}

\section{No. 1}

What is the best imaging strategy for acute stroke?

By Wardlaw JM, Keir SL, Seymour J, Lewis S, Sandercock PAG, Dennis MS, et al.

\section{No. 2}

Systematic review and modelling of the investigation of acute and chronic chest pain presenting in primary care.

By Mant J, McManus RJ, Oakes RAL, Delaney BC, Barton PM, Deeks JJ, et al.

\section{No. 3}

The effectiveness and cost-effectiveness of microwave and thermal balloon endometrial ablation for heavy menstrual bleeding: a systematic review and economic modelling.

By Garside R, Stein K, Wyatt K, Round A, Price A.

\section{No. 4}

A systematic review of the role of bisphosphonates in metastatic disease.

By Ross JR, Saunders Y,

Edmonds PM, Patel S, Wonderling D, Normand C, et al.

\section{No. 5}

Systematic review of the clinical effectiveness and cost-effectiveness of capecitabine (Xeloda ${ }^{\star}$ ) for locally advanced and/or metastatic breast cancer.

By Jones L, Hawkins N, Westwood M, Wright K, Richardson G, Riemsma R.

\section{No. 6}

Effectiveness and efficiency of guideline dissemination and implementation strategies.

By Grimshaw JM, Thomas RE, MacLennan G, Fraser C, Ramsay CR, Vale L, et al.

\section{No. 7}

Clinical effectiveness and costs of the Sugarbaker procedure for the treatment of pseudomyxoma peritonei

By Bryant J, Clegg AJ, Sidhu MK, Brodin H, Royle P, Davidson P.

\section{No. 8}

Psychological treatment for insomnia in the regulation of long-term hypnotic drug use.

By Morgan K, Dixon S, Mathers N, Thompson J, Tomeny M.

\section{No. 9}

Improving the evaluation of therapeutic interventions in multiple sclerosis: development of a patientbased measure of outcome.

By Hobart JC, Riazi A, Lamping DL, Fitzpatrick R, Thompson AJ.

\section{No. 10}

A systematic review and economic evaluation of magnetic resonance cholangiopancreatography compared with diagnostic endoscopic retrograde cholangiopancreatography.

By Kaltenthaler E, Bravo Vergel Y, Chilcott J, Thomas S, Blakeborough T, Walters SJ, et al.

No. 11

The use of modelling to evaluate new drugs for patients with a chronic condition: the case of antibodies against tumour necrosis factor in rheumatoid arthritis.

By Barton P, Jobanputra P, Wilson J, Bryan S, Burls A.

No. 12

Clinical effectiveness and costeffectiveness of neonatal screening for inborn errors of metabolism using tandem mass spectrometry: a systematic review.

By Pandor A, Eastham J, Beverley C, Chilcott J, Paisley S.

No. 13

Clinical effectiveness and costeffectiveness of pioglitazone and rosiglitazone in the treatment of type 2 diabetes: a systematic review and economic evaluation.

By Czoski-Murray C, Warren E, Chilcott J, Beverley C, Psyllaki MA Cowan J.

\section{No. 14}

Routine examination of the newborn: the EMREN study. Evaluation of an extension of the midwife role including a randomised controlled trial of appropriately trained midwives and paediatric senior house officers.

By Townsend J, Wolke D, Hayes J, Davé S, Rogers C, Bloomfield L, et al.

\section{No. 15}

Involving consumers in research and development agenda setting for the NHS: developing an evidence-based approach.

By Oliver S, Clarke-Jones L, Rees R, Milne R, Buchanan P, Gabbay J, et al.

\section{No. 16}

A multi-centre randomised controlled trial of minimally invasive direct coronary bypass grafting versus percutaneous transluminal coronary angioplasty with stenting for proximal stenosis of the left anterior descending coronary artery.

By Reeves BC, Angelini GD, Bryan AJ, Taylor FC, Cripps T, Spyt TJ, et al.

No. 17

Does early magnetic resonance imaging influence management or improve outcome in patients referred to secondary care with low back pain? A pragmatic randomised controlled trial.

By Gilbert FJ, Grant AM, Gillan MGC, Vale L, Scott NW, Campbell MK, et al. 


\section{No. 18}

The clinical and cost-effectivenes of anakinra for the treatment of rheumatoid arthritis in adults: a systematic review and economic analysis.

By Clark W, Jobanputra P, Barton P, Burls A

\section{No. 19}

A rapid and systematic review and economic evaluation of the clinical and cost-effectiveness of newer drugs for treatment of mania associated with bipolar affective disorder.

By Bridle C, Palmer S, Bagnall A-M, Darba J, Duffy S, Sculpher M, et al.

\section{No. 20}

Liquid-based cytology in cervical screening: an updated rapid and systematic review and economic analysis.

By Karnon J, Peters J, Platt J, Chilcott J, McGoogan E, Brewer N.

\section{No. 21}

Systematic review of the long-term effects and economic consequences of treatments for obesity and implications for health improvement.

By Avenell A, Broom J, Brown TJ, Poobalan A, Aucott L, Stearns SC, et al.

\section{No. 22}

Autoantibody testing in children with newly diagnosed type 1 diabetes mellitus.

By Dretzke J, Cummins C, Sandercock J, Fry-Smith A, Barrett T, Burls A.

\section{No. 23}

Clinical effectiveness and costeffectiveness of prehospital intravenous fluids in trauma patients.

By Dretzke J, Sandercock J, Bayliss S, Burls A.

\section{No. 24}

Newer hypnotic drugs for the shortterm management of insomnia: a systematic review and economic evaluation.

By Dündar Y, Boland A, Strobl J, Dodd S, Haycox A, Bagust A, et al.

\section{No. 25}

Development and validation of methods for assessing the quality of diagnostic accuracy studies.

By Whiting P, Rutjes AWS, Dinnes J, Reitsma JB, Bossuyt PMM, Kleijnen J.

\section{No. 26}

EVALUATE hysterectomy trial: a multicentre randomised trial comparing abdominal, vaginal and laparoscopic methods of hysterectomy.

By Garry R, Fountain J, Brown J, Manca A, Mason S, Sculpher M, et al.

\section{No. 27}

Methods for expected value of information analysis in complex health economic models: developments on the health economics of interferon- $\beta$ and glatiramer acetate for multiple sclerosis.

By Tappenden P, Chilcott JB, Eggington S, Oakley J, McCabe C.

\section{No. 28}

Effectiveness and cost-effectiveness of imatinib for first-line treatment of chronic myeloid leukaemia in chronic phase: a systematic review and economic analysis.

By Dalziel K, Round A, Stein K, Garside R, Price A.

\section{No. 29}

VenUS I: a randomised controlled trial of two types of bandage for treating venous leg ulcers.

By Iglesias C, Nelson EA, Cullum NA, Torgerson DJ, on behalf of the VenUS Team.

\section{No. 30}

Systematic review of the effectiveness and cost-effectiveness, and economic evaluation, of myocardial perfusion scintigraphy for the diagnosis and management of angina and myocardial infarction.

By Mowatt G, Vale L, Brazzelli M, Hernandez R, Murray A, Scott N, et al.

\section{No. 31}

A pilot study on the use of decision theory and value of information analysis as part of the NHS Health Technology Assessment programme.

By Claxton K, Ginnelly L, Sculpher M, Philips Z, Palmer S.

\section{No. 32}

The Social Support and Family Health Study: a randomised controlled trial and economic evaluation of two alternative forms of postnatal support for mothers living in disadvantaged inner-city areas.

By Wiggins M, Oakley A, Roberts I, Turner H, Rajan L, Austerberry H, et al.

\section{No. 33}

Psychosocial aspects of genetic screening of pregnant women and newborns: a systematic review.

By Green JM, Hewison J, Bekker HL, Bryant LD, Cuckle HS.

\section{No. 34}

Evaluation of abnormal uterine bleeding: comparison of three outpatient procedures within cohorts defined by age and menopausal status.

By Critchley HOD, Warner P, Lee AJ, Brechin S, Guise J, Graham B.

\section{No. 35}

Coronary artery stents: a rapid systematic review and economic evaluation.

By Hill R, Bagust A, Bakhai A

Dickson R, Dündar Y, Haycox A, et al.
No. 36

Review of guidelines for good practice in decision-analytic modelling in health technology assessment.

By Philips Z, Ginnelly L, Sculpher M, Claxton K, Golder S, Riemsma R, et al.

No. 37

Rituximab (MabThera ${ }^{\circledR}$ ) for aggressive non-Hodgkin's lymphoma: systematic review and economic evaluation.

By Knight C, Hind D, Brewer N, Abbott V.

\section{No. 38}

Clinical effectiveness and cost-

effectiveness of clopidogrel and modified-release dipyridamole in the secondary prevention of occlusive vascular events: a systematic review and economic evaluation.

By Jones L, Griffin S, Palmer S, Main C, Orton V, Sculpher M, et al.

No. 39

Pegylated interferon $\alpha$-2a and -2b in combination with ribavirin in the treatment of chronic hepatitis C: a systematic review and economic evaluation.

By Shepherd J, Brodin H, Cave C, Waugh N, Price A, Gabbay J.

\section{No. 40}

Clopidogrel used in combination with aspirin compared with aspirin alone in the treatment of non-ST-segmentelevation acute coronary syndromes: a systematic review and economic evaluation.

By Main C, Palmer S, Griffin S, Jones L, Orton V, Sculpher M, et al.

No. 41

Provision, uptake and cost of cardiac rehabilitation programmes: improving services to under-represented groups.

By Beswick AD, Rees K, Griebsch I, Taylor FC, Burke M, West RR, et al.

No. 42

Involving South Asian patients in clinical trials.

By Hussain-Gambles M, Leese B, Atkin K, Brown J, Mason S, Tovey P.

No. 43

Clinical and cost-effectiveness of continuous subcutaneous insulin infusion for diabetes.

By Colquitt JL, Green C, Sidhu MK, Hartwell D, Waugh N.

No. 44

Identification and assessment of ongoing trials in health technology assessment reviews.

By Song FJ, Fry-Smith A, Davenport C, Bayliss S, Adi Y, Wilson JS, et al.

No. 45

Systematic review and economic evaluation of a long-acting insulin analogue, insulin glargine

By Warren E, Weatherley-Jones E, Chilcott J, Beverley C. 


\section{No. 46}

Supplementation of a home-based exercise programme with a classbased programme for people with osteoarthritis of the knees: a randomised controlled trial and health economic analysis.

By McCarthy CJ, Mills PM, Pullen R, Richardson G, Hawkins N, Roberts CR, et al.

\section{No. 47}

Clinical and cost-effectiveness of oncedaily versus more frequent use of same potency topical corticosteroids for atopic eczema: a systematic review and economic evaluation.

By Green C, Colquitt JL, Kirby J, Davidson P, Payne E.

\section{No. 48}

Acupuncture of chronic headache disorders in primary care: randomised controlled trial and economic analysis.

By Vickers AJ, Rees RW, Zollman CE, McCarney R, Smith CM, Ellis N, et al.

\section{No. 49}

Generalisability in economic evaluation studies in healthcare: a review and case studies.

By Sculpher MJ, Pang FS, Manca A, Drummond MF, Golder S, Urdahl H, et al.

\section{No. 50}

Virtual outreach: a randomised controlled trial and economic evaluation of joint teleconferenced medical consultations.

By Wallace P, Barber J, Clayton W, Currell R, Fleming K, Garner P, et al.

\section{Volume 9, 2005}

\section{No. 1}

Randomised controlled multiple treatment comparison to provide a costeffectiveness rationale for the selection of antimicrobial therapy in acne.

By Ozolins M, Eady EA, Avery A, Cunliffe WJ, O'Neill C, Simpson NB, et al.

\section{No. 2}

Do the findings of case series studies vary significantly according to methodological characteristics?

By Dalziel K, Round A, Stein K, Garside R, Castelnuovo E, Payne L.

\section{No. 3}

Improving the referral process for familial breast cancer genetic counselling: findings of three randomised controlled trials of two interventions.

By Wilson BJ, Torrance N,

\section{No. 4}

Randomised evaluation of alternative electrosurgical modalities to treat bladder outflow obstruction in men with benign prostatic hyperplasia.

By Fowler C, McAllister W, Plail R, Karim $O$, Yang $Q$.

\section{No. 5}

A pragmatic randomised controlled trial of the cost-effectiveness of palliative therapies for patients with inoperable oesophageal cancer.

By Shenfine J, McNamee P, Steen N, Bond J, Griffin SM.

\section{No. 6}

Impact of computer-aided detection prompts on the sensitivity and specificity of screening mammography.

By Taylor P, Champness J, GivenWilson R, Johnston K, Potts H.

\section{No. 7}

Issues in data monitoring and interim analysis of trials.

By Grant AM, Altman DG, Babiker $\mathrm{AB}$, Campbell MK, Clemens FJ, Darbyshire JH, et al.

\section{No. 8}

Lay public's understanding of equipoise and randomisation in randomised controlled trials.

By Robinson EJ, Kerr CEP, Stevens AJ, Lilford RJ, Braunholtz DA, Edwards SJ, et al.

\section{No. 9}

Clinical and cost-effectiveness of electroconvulsive therapy for depressive illness, schizophrenia, catatonia and mania: systematic reviews and economic modelling studies.

By Greenhalgh J, Knight C, Hind D, Beverley C, Walters S.

\section{No. 10}

Measurement of health-related quality of life for people with dementia: development of a new instrument (DEMQOL) and an evaluation of current methodology.

By Smith SC, Lamping DL, Banerjee S, Harwood R, Foley B, Smith P, et al.

No. 11

Clinical effectiveness and costeffectiveness of drotrecogin alfa (activated) (Xigris ${ }^{\circledR}$ ) for the treatment of severe sepsis in adults: a systematic review and economic evaluation.

By Green C, Dinnes J, Takeda A, Shepherd J, Hartwell D, Cave C, et al.

\section{No. 12}

A methodological review of how heterogeneity has been examined in systematic reviews of diagnostic test accuracy.

By Dinnes J, Deeks J, Kirby J, Roderick P.
No. 13

Cervical screening programmes: can automation help? Evidence from systematic reviews, an economic analysis and a simulation modelling exercise applied to the UK.

By Willis BH, Barton P, Pearmain P, Bryan S, Hyde C.

\section{No. 14}

Laparoscopic surgery for inguinal hernia repair: systematic review of effectiveness and economic evaluation. By McCormack K, Wake B, Perez J, Fraser C, Cook J, McIntosh E, et al.

\section{No. 15}

Clinical effectiveness, tolerability and cost-effectiveness of newer drugs for epilepsy in adults: a systematic review and economic evaluation.

By Wilby J, Kainth A, Hawkins N, Epstein D, McIntosh H, McDaid C, et al.

\section{No. 16}

A randomised controlled trial to compare the cost-effectiveness of tricyclic antidepressants, selective serotonin reuptake inhibitors and lofepramine

By Peveler R, Kendrick T, Buxton M, Longworth L, Baldwin D, Moore M, et al.

\section{No. 17}

Clinical effectiveness and costeffectiveness of immediate angioplasty for acute myocardial infarction: systematic review and economic evaluation.

By Hartwell D, Colquitt J, Loveman $\mathrm{E}$, Clegg AJ, Brodin $\mathrm{H}$, Waugh $\mathrm{N}$, et al.

No. 18

A randomised controlled comparison of alternative strategies in stroke care.

By Kalra L, Evans A, Perez I,

Knapp M, Swift C, Donaldson N.

No. 19

The investigation and analysis of critical incidents and adverse events in healthcare.

By Woloshynowych M, Rogers S, Taylor-Adams S, Vincent C.

No. 20

Potential use of routine databases in health technology assessment.

By Raftery J, Roderick P, Stevens A

No. 21

Clinical and cost-effectiveness of newer immunosuppressive regimens in renal transplantation: a systematic review and modelling study.

By Woodroffe R, Yao GL, Meads C, Bayliss S, Ready A, Raftery J, et al.

No. 22

A systematic review and economic evaluation of alendronate, etidronate, risedronate, raloxifene and teriparatide for the prevention and treatment of postmenopausal osteoporosis.

By Stevenson M, Lloyd Jones M, De Nigris E, Brewer N, Davis S, Oakley J. 


\section{No. 23}

A systematic review to examine the impact of psycho-educational interventions on health outcomes and costs in adults and children with difficult asthma.

By Smith JR, Mugford M, Holland R, Candy B, Noble MJ, Harrison BDW, et al.

\section{No. 24}

An evaluation of the costs, effectiveness and quality of renal replacement therapy provision in renal satellite units in England and Wales.

By Roderick P, Nicholson T, Armitage A, Mehta R, Mullee M, Gerard K, et al.

\section{No. 25}

Imatinib for the treatment of patients with unresectable and/or metastatic gastrointestinal stromal tumours: systematic review and economic evaluation.

By Wilson J, Connock M, Song F, Yao G, Fry-Smith A, Raftery J, et al.

\section{No. 26}

Indirect comparisons of competing interventions.

By Glenny AM, Altman DG, Song F, Sakarovitch C, Deeks JJ, D'Amico R, et al.

\section{No. 27}

Cost-effectiveness of alternative strategies for the initial medical management of non-ST elevation acute coronary syndrome: systematic review and decision-analytical modelling.

By Robinson M, Palmer S, Sculpher M, Philips Z, Ginnelly L, Bowens A, et al.

No. 28

Outcomes of electrically stimulated gracilis neosphincter surgery.

By Tillin T, Chambers M, Feldman R.

No. 29

The effectiveness and cost-effectiveness of pimecrolimus and tacrolimus for atopic eczema: a systematic review and economic evaluation.

By Garside R, Stein K, Castelnuovo E, Pitt M, Ashcroft D, Dimmock P, et al.

\section{No. 30}

Systematic review on urine albumin testing for early detection of diabetic complications.

By Newman DJ, Mattock MB, Dawnay ABS, Kerry S, McGuire A, Yaqoob M, et al.

No. 31

Randomised controlled trial of the costeffectiveness of water-based therapy for lower limb osteoarthritis.

By Cochrane T, Davey RC, Matthes Edwards SM.

\section{No. 32}

Longer term clinical and economic benefits of offering acupuncture care to patients with chronic low back pain.

By Thomas KJ, MacPherson H, Ratcliffe J, Thorpe L, Brazier J, Campbell M, et al.

\section{No. 33}

Cost-effectiveness and safety of epidural steroids in the management of sciatica.

By Price C, Arden N, Coglan L, Rogers P.

\section{No. 34}

The British Rheumatoid Outcome Study Group (BROSG) randomised controlled trial to compare the effectiveness and cost-effectiveness of aggressive versus symptomatic therapy in established rheumatoid arthritis.

By Symmons D, Tricker K, Roberts C, Davies L, Dawes P, Scott DL.

\section{No. 35}

Conceptual framework and systematic review of the effects of participants' and professionals' preferences in randomised controlled trials.

By King M, Nazareth I, Lampe F, Bower P, Chandler M, Morou M, et al.

\section{No. 36}

The clinical and cost-effectiveness of implantable cardioverter defibrillators: a systematic review.

By Bryant J, Brodin H, Loveman E Payne E, Clegg A.

\section{No. 37}

A trial of problem-solving by community mental health nurses for anxiety, depression and life difficulties among general practice patients. The CPN-GP study.

By Kendrick T, Simons L, Mynors-Wallis L, Gray A, Lathlean J, Pickering R, et al.

\section{No. 38}

The causes and effects of sociodemographic exclusions from clinical trials.

By Bartlett C, Doyal L, Ebrahim S Davey P, Bachmann M, Egger M, et al.

\section{No. 39}

Is hydrotherapy cost-effective? A randomised controlled trial of combined hydrotherapy programmes compared with physiotherapy land techniques in children with juvenile idiopathic arthritis.

By Epps H, Ginnelly L, Utley M, Southwood T, Gallivan S, Sculpher M, et al.

\section{No. 40}

A randomised controlled trial and cost-effectiveness study of systematic screening (targeted and total population screening) versus routine practice for the detection of atrial fibrillation in people aged 65 and over. The SAFE study.

By Hobbs FDR, Fitzmaurice DA, Mant J, Murray E, Jowett S, Bryan S, et al.

\section{No. 41}

Displaced intracapsular hip fractures in fit, older people: a randomised comparison of reduction and fixation, bipolar hemiarthroplasty and total hip arthroplasty.

By Keating JF, Grant A, Masson M, Scott NW, Forbes JF.

\section{No. 42}

Long-term outcome of cognitive behaviour therapy clinical trials in central Scotland.

By Durham RC, Chambers JA, Power KG, Sharp DM, Macdonald RR, Major KA, et al.

\section{No. 43}

The effectiveness and cost-effectiveness of dual-chamber pacemakers compared with single-chamber pacemakers for bradycardia due to atrioventricular block or sick sinus syndrome: systematic review and economic evaluation.

By Castelnuovo E, Stein K, Pitt M, Garside R, Payne E.

\section{No. 44}

Newborn screening for congenital heart defects: a systematic review and costeffectiveness analysis.

By Knowles R, Griebsch I, Dezateux C, Brown J, Bull C, Wren C.

\section{No. 45}

The clinical and cost-effectiveness of left ventricular assist devices for endstage heart failure: a systematic review and economic evaluation.

By Clegg AJ, Scott DA, Loveman E, Colquitt J, Hutchinson J, Royle P, et al.

\section{No. 46}

The effectiveness of the Heidelberg Retina Tomograph and laser diagnostic glaucoma scanning system (GDx) in detecting and monitoring glaucoma.

By Kwartz AJ, Henson DB, Harper RA, Spencer AF, McLeod D.

No. 47

Clinical and cost-effectiveness of autologous chondrocyte implantation for cartilage defects in knee joints: systematic review and economic evaluation.

By Clar C, Cummins E, McIntyre L, Thomas S, Lamb J, Bain L, et al. 


\section{No. 48}

Systematic review of effectiveness of different treatments for childhood retinoblastoma.

By McDaid C, Hartley S, Bagnall A-M, Ritchie G, Light K, Riemsma R.

\section{No. 49}

Towards evidence-based guidelines for the prevention of venous thromboembolism: systematic reviews of mechanical methods, oral anticoagulation, dextran and regional anaesthesia as thromboprophylaxis.

By Roderick P, Ferris G, Wilson K, Halls H, Jackson D, Collins R, et al.

\section{No. 50}

The effectiveness and cost-effectiveness of parent training/education programmes for the treatment of conduct disorder, including oppositional defiant disorder, in children.

By Dretzke J, Frew E, Davenport C, Barlow J, Stewart-Brown S, Sandercock J. et al.

\section{Volume 10, 2006}

\section{No. 1}

The clinical and cost-effectiveness of donepezil, rivastigmine, galantamine and memantine for Alzheimer's disease.

By Loveman E, Green C, Kirby J, Takeda A, Picot J, Payne E, et al.

\section{No. 2}

FOOD: a multicentre randomised trial evaluating feeding policies in patients admitted to hospital with a recent stroke.

By Dennis M, Lewis S, Cranswick G, Forbes J

No. 3

The clinical effectiveness and costeffectiveness of computed tomography screening for lung cancer: systematic reviews.

By Black C, Bagust A, Boland A, Walker S, McLeod C, De Verteuil R, et al.

\section{No. 4}

A systematic review of the effectiveness and cost-effectiveness of neuroimaging assessments used to visualise the seizure focus in people with refractory epilepsy being considered for surgery.

By Whiting P, Gupta R, Burch J, Mujica Mota RE, Wright K, Marson A, et al.

\section{No. 5}

Comparison of conference abstracts and presentations with full-text articles in the health technology assessments of rapidly evolving technologies.

By Dundar Y, Dodd S, Dickson R, Walley T, Haycox A, Williamson PR.

\section{No. 6}

Systematic review and evaluation of methods of assessing urinary incontinence.

By Martin JL, Williams KS, Abrams KR, Turner DA, Sutton AJ, Chapple C, et al.

\section{No. 7}

The clinical effectiveness and costeffectiveness of newer drugs for children with epilepsy. A systematic review.

By Connock M, Frew E, Evans B-W, Bryan S, Cummins C, Fry-Smith A, et al.

No. 8

Surveillance of Barrett's oesophagus: exploring the uncertainty through systematic review, expert workshop and economic modelling.

By Garside R, Pitt M, Somerville M, Stein K, Price A, Gilbert N.

\section{No. 9}

Topotecan, pegylated liposomal doxorubicin hydrochloride and paclitaxel for second-line or subsequent treatment of advanced ovarian cancer: a systematic review and economic evaluation.

By Main C, Bojke L, Griffin S, Norman G, Barbieri M, Mather L, et al.

\section{No. 10}

Evaluation of molecular techniques in prediction and diagnosis of cytomegalovirus disease in immunocompromised patients.

By Szczepura A, Westmoreland D, Vinogradova Y, Fox J, Clark M.

\section{No. 11}

Screening for thrombophilia in highrisk situations: systematic review and cost-effectiveness analysis. The Thrombosis: Risk and Economic Assessment of Thrombophilia

Screening (TREATS) study.

By Wu O, Robertson L, Twaddle S, Lowe GDO, Clark P, Greaves M, et al.

\section{No. 12}

A series of systematic reviews to inform a decision analysis for sampling and treating infected diabetic foot ulcers.

By Nelson EA, O'Meara S, Craig D, Iglesias C, Golder S, Dalton J, et al.

\section{No. 13}

Randomised clinical trial, observational study and assessment of cost-

effectiveness of the treatment of varicose veins (REACTIV trial).

By Michaels JA, Campbell WB, Brazier JE, MacIntyre JB, Palfreyman SJ, Ratcliffe J, et al.

\section{No. 14}

The cost-effectiveness of screening for oral cancer in primary care.

By Speight PM, Palmer S, Moles DR, Downer MC, Smith DH, Henriksson M, et al.

\section{No. 15}

Measurement of the clinical and costeffectiveness of non-invasive diagnostic testing strategies for deep vein thrombosis.

By Goodacre S, Sampson F, Stevenson M, Wailoo A, Sutton A Thomas S, et al.

\section{No. 16}

Systematic review of the effectiveness and cost-effectiveness of HealOzone for the treatment of occlusal pit/fissure caries and root caries.

By Brazzelli M, McKenzie L, Fielding S, Fraser C, Clarkson J, Kilonzo M, et al.

\section{No. 17}

Randomised controlled trials of conventional antipsychotic versus new atypical drugs, and new atypical drugs versus clozapine, in people with schizophrenia responding poorly to, or intolerant of, current drug treatment.

By Lewis SW, Davies L, Jones PB, Barnes TRE, Murray RM, Kerwin R, et al.

\section{No. 18}

Diagnostic tests and algorithms used in the investigation of haematuria: systematic reviews and economic evaluation.

By Rodgers M, Nixon J, Hempel S, Aho T, Kelly J, Neal D, et al.

\section{No. 19}

Cognitive behavioural therapy in addition to antispasmodic therapy for irritable bowel syndrome in primary care: randomised controlled trial.

By Kennedy TM, Chalder T, McCrone P, Darnley S, Knapp M, Jones RH, et al.

\section{No. 20}

A systematic review of the clinical effectiveness and costeffectiveness of enzyme replacement therapies for Fabry's disease and mucopolysaccharidosis type 1 .

By Connock M, Juarez-Garcia A, Frew E, Mans A, Dretzke J, Fry-Smith A, et al.

\section{No. 21}

Health benefits of antiviral therapy for mild chronic hepatitis $\mathrm{C}$ : randomised controlled trial and economic evaluation

By Wright M, Grieve R, Roberts J, Main J, Thomas HC, on behalf of the UK Mild Hepatitis C Trial Investigators.

No. 22

Pressure relieving support surfaces: a randomised evaluation.

By Nixon J, Nelson EA, Cranny G, Iglesias CP, Hawkins K, Cullum NA, et al. 
No. 23

A systematic review and economic model of the effectiveness and costeffectiveness of methylphenidate, dexamfetamine and atomoxetine for the treatment of attention deficit hyperactivity disorder in children and adolescents.

By King S, Griffin S, Hodges Z, Weatherly H, Asseburg C, Richardson G, et al.

\section{No. 24}

The clinical effectiveness and costeffectiveness of enzyme replacement therapy for Gaucher's disease: a systematic review.

By Connock M, Burls A, Frew E, Fry-Smith A, Juarez-Garcia A, McCabe C, et al.

\section{No. 25}

Effectiveness and cost-effectiveness of salicylic acid and cryotherapy for cutaneous warts. An economic decision model.

By Thomas KS, Keogh-Brown MR, Chalmers JR, Fordham RJ, Holland RC, Armstrong SJ, et al.

\section{No. 26}

A systematic literature review of the effectiveness of non-pharmacological interventions to prevent wandering in dementia and evaluation of the ethical implications and acceptability of their use.

By Robinson L, Hutchings D, Corner L, Beyer F, Dickinson H, Vanoli A, et al.

\section{No. 27}

A review of the evidence on the effects and costs of implantable cardioverter defibrillator therapy in different patient groups, and modelling of costeffectiveness and cost-utility for these groups in a UK context.

By Buxton M, Caine N, Chase D, Connelly D, Grace A, Jackson C, et al.

\section{No. 28}

Adefovir dipivoxil and pegylated interferon alfa-2a for the treatment of chronic hepatitis B: a systematic review and economic evaluation.

By Shepherd J, Jones J, Takeda A, Davidson P, Price A.

\section{No. 29}

An evaluation of the clinical and costeffectiveness of pulmonary artery catheters in patient management in intensive care: a systematic review and a randomised controlled trial.

By Harvey S, Stevens K, Harrison D, Young D, Brampton W, McCabe C, et al.

\section{No. 30}

Accurate, practical and cost-effective assessment of carotid stenosis in the UK.

By Wardlaw JM, Chappell FM Stevenson M, De Nigris E, Thomas S, Gillard J, et al.

\section{No. 31}

Etanercept and infliximab for the treatment of psoriatic arthritis: a systematic review and economic evaluation.

By Woolacott N, Bravo Vergel Y, Hawkins N, Kainth A, Khadjesari Z, Misso K, et al.

\section{No. 32}

The cost-effectiveness of testing for hepatitis $\mathrm{C}$ in former injecting drug users.

By Castelnuovo E, Thompson-Coon J, Pitt M, Cramp M, Siebert U, Price A, et al.

\section{No. 33}

Computerised cognitive behaviour therapy for depression and anxiety update: a systematic review and economic evaluation.

By Kaltenthaler E, Brazier J, De Nigris E, Tumur I, Ferriter M, Beverley C, et al.

No. 34

Cost-effectiveness of using prognostic information to select women with breast cancer for adjuvant systemic therapy.

By Williams C, Brunskill S, Altman D, Briggs A, Campbell H, Clarke M, et al.

No. 35

Psychological therapies including dialectical behaviour therapy for borderline personality disorder: a systematic review and preliminary economic evaluation.

By Brazier J, Tumur I, Holmes M, Ferriter M, Parry G, Dent-Brown K, et al.

\section{No. 36}

Clinical effectiveness and costeffectiveness of tests for the diagnosis and investigation of urinary tract infection in children: a systematic review and economic model.

By Whiting P, Westwood M, Bojke L, Palmer S, Richardson G, Cooper J, et al.

\section{No. 37}

Cognitive behavioural therapy in chronic fatigue syndrome: a randomised controlled trial of an outpatient group programme. By O’Dowd H, Gladwell P, Rogers CA, Hollinghurst S, Gregory A.

\section{No. 38}

A comparison of the cost-effectiveness of five strategies for the prevention of nonsteroidal anti-inflammatory drug-induced gastrointestinal toxicity: a systematic review with economic modelling.

By Brown TJ, Hooper L, Elliott RA, Payne K, Webb R, Roberts C, et al.

\section{No. 39}

The effectiveness and cost-effectiveness of computed tomography screening for coronary artery disease: systematic review.

By Waugh N, Black C, Walker S, McIntyre L, Cummins E, Hillis G.

\section{No. 40}

What are the clinical outcome and costeffectiveness of endoscopy undertaken by nurses when compared with doctors? A Multi-Institution Nurse Endoscopy Trial (MINuET).

By Williams J, Russell I, Durai D, Cheung W-Y, Farrin A, Bloor K, et al.

No. 41

The clinical and cost-effectiveness of oxaliplatin and capecitabine for the adjuvant treatment of colon cancer: systematic review and economic evaluation.

By Pandor A, Eggington S, Paisley S, Tappenden P, Sutcliffe P.

\section{No. 42}

A systematic review of the effectiveness of adalimumab, etanercept and infliximab for the treatment of rheumatoid arthritis in adults and an economic evaluation of their costeffectiveness.

By Chen Y-F, Jobanputra P, Barton P, Jowett S, Bryan S, Clark W, et al.

\section{No. 43}

Telemedicine in dermatology: a randomised controlled trial.

By Bowns IR, Collins K, Walters SJ McDonagh AJG.

\section{No. 44}

Cost-effectiveness of cell salvage and alternative methods of minimising perioperative allogeneic blood transfusion: a systematic review and economic model.

By Davies L, Brown TJ, Haynes S, Payne K, Elliott RA, McCollum C.

\section{No. 45}

Clinical effectiveness and costeffectiveness of laparoscopic surgery for colorectal cancer: systematic reviews and economic evaluation.

By Murray A, Lourenco T, de Verteuil R, Hernandez R, Fraser C, McKinley A, et al.

\section{No. 46}

Etanercept and efalizumab for the treatment of psoriasis: a systematic review.

By Woolacott N, Hawkins N, Mason A, Kainth A, Khadjesari Z, Bravo Vergel Y, et al.

\section{No. 47}

Systematic reviews of clinical decision tools for acute abdominal pain

By Liu JLY, Wyatt JC, Deeks JJ, Clamp S, Keen J, Verde P, et al.

\section{No. 48}

Evaluation of the ventricular assist device programme in the UK.

By Sharples L, Buxton M, Caine N, Cafferty F, Demiris N, Dyer M, et al. 


\section{No. 49}

A systematic review and economic model of the clinical and costeffectiveness of immunosuppressive therapy for renal transplantation in children.

By Yao G, Albon E, Adi Y, Milford D, Bayliss S, Ready A, et al.

\section{No. 50}

Amniocentesis results: investigation of anxiety. The ARIA trial.

By Hewison J, Nixon J, Fountain J, Cocks K, Jones C, Mason G, et al.

\section{Volume II, 2007}

\section{No. 1}

Pemetrexed disodium for the treatment of malignant pleural mesothelioma: a systematic review and economic evaluation.

By Dundar Y, Bagust A, Dickson R, Dodd S, Green J, Haycox A, et al.

\section{No. 2}

A systematic review and economic model of the clinical effectiveness and cost-effectiveness of docetaxel in combination with prednisone or prednisolone for the treatment of hormone-refractory metastatic prostate cancer.

By Collins R, Fenwick E, Trowman R, Perard R, Norman G, Light K, et al.

\section{No. 3}

A systematic review of rapid diagnostic tests for the detection of tuberculosis infection.

By Dinnes J, Deeks J, Kunst H, Gibson A, Cummins E, Waugh N, et al.

\section{No. 4}

The clinical effectiveness and costeffectiveness of strontium ranelate for the prevention of osteoporotic fragility fractures in postmenopausal women.

By Stevenson M, Davis S, Lloyd-Jones M, Beverley C.

\section{No. 5}

A systematic review of quantitative and qualitative research on the role and effectiveness of written information available to patients about individual medicines.

By Raynor DK, Blenkinsopp A, Knapp P, Grime J, Nicolson DJ, Pollock K, et al.

No. 6

Oral naltrexone as a treatment for relapse prevention in formerly opioiddependent drug users: a systematic review and economic evaluation.

By Adi Y, Juarez-Garcia A, Wang D, Jowett S, Frew E, Day E, et al.

\section{No. 7}

Glucocorticoid-induced osteoporosis: a systematic review and cost-utility analysis.

By Kanis JA, Stevenson M, McCloskey EV, Davis S, Lloyd-Jones M

No. 8

Epidemiological, social, diagnostic and economic evaluation of population screening for genital chlamydial infection.

By Low N, McCarthy A, Macleod J, Salisbury C, Campbell R, Roberts TE, et al.

\section{No. 9}

Methadone and buprenorphine for the management of opioid dependence: a systematic review and economic evaluation.

By Connock M, Juarez-Garcia A, Jowett S, Frew E, Liu Z, Taylor RJ, et al.

\section{No. 10}

Exercise Evaluation Randomised Trial (EXERT): a randomised trial comparing GP referral for leisure centre-based exercise, community-based walking and advice only.

By Isaacs AJ, Critchley JA, See Tai

S, Buckingham K, Westley D, Harridge $\mathrm{SDR}$, et al.

\section{No. 11}

Interferon alfa (pegylated and nonpegylated) and ribavirin for the treatment of mild chronic hepatitis C: a systematic review and economic evaluation.

By Shepherd J, Jones J, Hartwell D, Davidson P, Price A, Waugh N.

\section{No. 12}

Systematic review and economic evaluation of bevacizumab and cetuximab for the treatment of metastatic colorectal cancer.

By Tappenden P, Jones R, Paisley S, Carroll C.

No. 13

A systematic review and economic evaluation of epoetin alfa, epoetin beta and darbepoetin alfa in anaemia associated with cancer, especially that attributable to cancer treatment.

By Wilson J, Yao GL, Raftery J, Bohlius J, Brunskill S, Sandercock J, et al.

No. 14

A systematic review and economic evaluation of statins for the prevention of coronary events.

By Ward S, Lloyd Jones M, Pandor A, Holmes M, Ara R, Ryan A, et al.

No. 15

A systematic review of the effectiveness and cost-effectiveness of different models of community-based respite care for frail older people and their carers.

By Mason A, Weatherly H, Spilsbury $\mathrm{K}$, Arksey H, Golder S, Adamson J, et al.
No. 16

Additional therapy for young children with spastic cerebral palsy: a randomised controlled trial.

By Weindling AM, Cunningham CC, Glenn SM, Edwards RT, Reeves DJ.

\section{No. 17}

Screening for type 2 diabetes: literature review and economic modelling.

By Waugh N, Scotland G, McNamee P, Gillett M, Brennan A, Goyder E, et al.

\section{No. 18}

The effectiveness and cost-effectiveness of cinacalcet for secondary

hyperparathyroidism in end-stage renal disease patients on dialysis: a systematic review and economic evaluation.

By Garside R, Pitt M, Anderson R, Mealing S, Roome C, Snaith A, et al.

\section{No. 19}

The clinical effectiveness and costeffectiveness of gemcitabine for metastatic breast cancer: a systematic review and economic evaluation.

By Takeda AL, Jones J, Loveman E, Tan SC, Clegg AJ.

No. 20

A systematic review of duplex ultrasound, magnetic resonance angiography and computed tomography angiography for the diagnosis and assessment of symptomatic, lower limb peripheral arterial disease.

By Collins R, Cranny G, Burch J, Aguiar-Ibáñez R, Craig D, Wright K, et al.

No. 21

The clinical effectiveness and costeffectiveness of treatments for children with idiopathic steroid-resistant nephrotic syndrome: a systematic review.

By Colquitt JL, Kirby J, Green C, Cooper K, Trompeter RS.

No. 22

A systematic review of the routine monitoring of growth in children of primary school age to identify growthrelated conditions.

By Fayter D, Nixon J, Hartley S, Rithalia A, Butler G, Rudolf M, et al.

\section{No. 23}

Systematic review of the effectiveness of preventing and treating Staphylococcus aureus carriage in reducing peritoneal catheter-related infections.

By McCormack K, Rabindranath K, Kilonzo M, Vale L, Fraser C, McIntyre L, et al. 
No. 24

The clinical effectiveness and cost of repetitive transcranial magnetic stimulation versus electroconvulsive therapy in severe depression: a multicentre pragmatic randomised controlled trial and economic analysis.

By McLoughlin DM, Mogg A, Eranti S, Pluck G, Purvis R, Edwards D, et al

No. 25

A randomised controlled trial and economic evaluation of direct versus indirect and individual versus group modes of speech and language therapy for children with primary language impairment.

By Boyle J, McCartney E, Forbes J, O'Hare A.

\section{No. 26}

Hormonal therapies for early breast cancer: systematic review and economic evaluation.

By Hind D, Ward S, De Nigris E, Simpson E, Carroll C, Wyld L.

\section{No. 27}

Cardioprotection against the toxic effects of anthracyclines given to children with cancer: a systematic review.

By Bryant J, Picot J, Levitt G, Sullivan I, Baxter L, Clegg A.

\section{No. 28}

Adalimumab, etanercept and infliximab for the treatment of ankylosing spondylitis: a systematic review and economic evaluation.

By McLeod C, Bagust A, Boland A, Dagenais P, Dickson R, Dundar Y, et al.

\section{No. 29}

Prenatal screening and treatment strategies to prevent group B streptococcal and other bacterial infections in early infancy: costeffectiveness and expected value of information analyses.

By Colbourn T, Asseburg C, Bojke L, Philips Z, Claxton K, Ades AE, et al.

No. 30

Clinical effectiveness and costeffectiveness of bone morphogenetic proteins in the non-healing of fractures and spinal fusion: a systematic review.

By Garrison KR, Donell S, Ryder J, Shemilt I, Mugford M, Harvey I, et al.

No. 31

A randomised controlled trial of postoperative radiotherapy following breast-conserving surgery in a minimum-risk older population. The PRIME trial.

By Prescott RJ, Kunkler IH, Williams LJ, King CC, Jack W, van der Pol M, et al.

\section{No. 32}

Current practice, accuracy, effectiveness and cost-effectiveness of the school entry hearing screen.

By Bamford J, Fortnum H, Bristow K, Smith J, Vamvakas G, Davies L, et al.

\section{No. 33}

The clinical effectiveness and costeffectiveness of inhaled insulin in diabetes mellitus: a systematic review and economic evaluation.

By Black C, Cummins E, Royle P, Philip S, Waugh N.

\section{No. 34}

Surveillance of cirrhosis for hepatocellular carcinoma: systematic review and economic analysis.

By Thompson Coon J, Rogers G, Hewson P, Wright D, Anderson R, Cramp M, et al.

\section{No. 35}

The Birmingham Rehabilitation Uptake Maximisation Study (BRUM). Homebased compared with hospitalbased cardiac rehabilitation in a multiethnic population: cost-effectiveness and patient adherence.

By Jolly K, Taylor R, Lip GYH, Greenfield S, Raftery J, Mant J, et al.

\section{No. 36}

A systematic review of the clinical, public health and cost-effectiveness of rapid diagnostic tests for the detection and identification of bacterial intestinal pathogens in faeces and food.

By Abubakar I, Irvine L, Aldus CF, Wyatt GM, Fordham R, Schelenz S, et al.

\section{No. 37}

A randomised controlled trial examining the longer-term outcomes of standard versus new antiepileptic drugs. The SANAD trial.

By Marson AG, Appleton R, Baker GA, Chadwick DW, Doughty J, Eaton B et al.

\section{No. 38}

Clinical effectiveness and costeffectiveness of different models of managing long-term oral anticoagulation therapy: a systematic review and economic modelling.

By Connock M, Stevens C, Fry-Smith A, Jowett S, Fitzmaurice D, Moore D, et al.

\section{No. 39}

A systematic review and economic model of the clinical effectiveness and cost-effectiveness of interventions for preventing relapse in people with bipolar disorder.

By Soares-Weiser K, Bravo Vergel Y, Beynon S, Dunn G, Barbieri M, Duffy S, et al.

\section{No. 40}

Taxanes for the adjuvant treatment of early breast cancer: systematic review and economic evaluation.

By Ward S, Simpson E, Davis S, Hind D, Rees A, Wilkinson A.

\section{No. 41}

The clinical effectiveness and costeffectiveness of screening for open angle glaucoma: a systematic review and economic evaluation.

By Burr JM, Mowatt G, Hernández R, Siddiqui MAR, Cook J, Lourenco T, et al.

\section{No. 42}

Acceptability, benefit and costs of early screening for hearing disability: a study of potential screening tests and models.

By Davis A, Smith P, Ferguson M, Stephens D, Gianopoulos I.

\section{No. 43}

Contamination in trials of educational interventions.

By Keogh-Brown MR, Bachmann

MO, Shepstone L, Hewitt C, Howe A, Ramsay CR, et al.

\section{No. 44}

Overview of the clinical effectiveness of positron emission tomography imaging in selected cancers.

By Facey K, Bradbury I, Laking G, Payne E.

\section{No. 45}

The effectiveness and cost-effectiveness of carmustine implants and temozolomide for the treatment of newly diagnosed high-grade glioma: a systematic review and economic evaluation.

By Garside R, Pitt M, Anderson R, Rogers G, Dyer M, Mealing S, et al.

\section{No. 46}

Drug-eluting stents: a systematic review and economic evaluation.

By Hill RA, Boland A, Dickson R, Dündar Y, Haycox A, McLeod C, et al.

\section{No. 47}

The clinical effectiveness and cost-effectiveness of cardiac resynchronisation (biventricular pacing) for heart failure: systematic review and economic model.

By Fox M, Mealing S, Anderson R, Dean J, Stein K, Price A, et al.

\section{No. 48}

Recruitment to randomised trials: strategies for trial enrolment and participation study. The STEPS study.

By Campbell MK, Snowdon C, Francis D, Elbourne D, McDonald AM, Knight R, et al.

\section{No. 49}

Cost-effectiveness of functiona cardiac testing in the diagnosis and management of coronary artery disease: a randomised controlled trial. The CECaT trial.

By Sharples L, Hughes V, Crean A, Dyer M, Buxton M, Goldsmith K, et al. 
No. 50

Evaluation of diagnostic tests when there is no gold standard. A review of methods.

By Rutjes AWS, Reitsma

JB, Coomarasamy A, Khan KS, Bossuyt PMM.

\section{No. 51}

Systematic reviews of the clinical effectiveness and cost-effectiveness of proton pump inhibitors in acute upper gastrointestinal bleeding

By Leontiadis GI, Sreedharan A, Dorward S, Barton P, Delaney B, Howden CW, et al.

\section{No. 52}

A review and critique of modelling in prioritising and designing screening programmes.

By Karnon J, Goyder E, Tappenden P, McPhie S, Towers I, Brazier J, et al.

\section{No. 53}

An assessment of the impact of the NHS Health Technology Assessment Programme.

By Hanney S, Buxton M, Green C, Coulson D, Raftery J.

\section{Volume 12, 2008}

\section{No. 1}

A systematic review and economic model of switching from nonglycopeptide to glycopeptide antibiotic prophylaxis for surgery.

By Cranny G, Elliott R, Weatherly H, Chambers D, Hawkins N, Myers L, et al.

\section{No. 2}

'Cut down to quit' with nicotine replacement therapies in smoking cessation: a systematic review of effectiveness and economic analysis.

By Wang D, Connock M, Barton P, Fry-Smith A, Aveyard P, Moore D.

\section{No. 3}

A systematic review of the effectiveness of strategies for reducing fracture risk in children with juvenile idiopathic arthritis with additional data on longterm risk of fracture and cost of disease management.

By Thornton J, Ashcroft D, O'Neill T, Elliott R, Adams J, Roberts C, et al.

\section{No. 4}

Does befriending by trained lay workers improve psychological well-being and quality of life for carers of people with dementia, and at what cost? A randomised controlled trial.

By Charlesworth G, Shepstone L,
No. 5

A multi-centre retrospective cohort study comparing the efficacy, safety and cost-effectiveness of hysterectomy and uterine artery embolisation for the treatment of symptomatic uterine fibroids. The HOPEFUL study.

By Hirst A, Dutton S, Wu O, Briggs A, Edwards C, Waldenmaier L, et al.

\section{No. 6}

Methods of prediction and prevention of pre-eclampsia: systematic reviews of accuracy and effectiveness literature with economic modelling.

By Meads CA, Cnossen JS, Meher S, Juarez-Garcia A, ter Riet G, Duley L, et al.

\section{No. 7}

The use of economic evaluations in NHS decision-making: a review and empirical investigation.

By Williams I, McIver S, Moore D, Bryan S.

\section{No. 8}

Stapled haemorrhoidectomy

(haemorrhoidopexy) for the treatment of haemorrhoids: a systematic review and economic evaluation.

By Burch J, Epstein D, Baba-Akbari A, Weatherly H, Fox D, Golder S, et al.

\section{No. 9}

The clinical effectiveness of diabetes education models for Type 2 diabetes: a systematic review.

By Loveman E, Frampton GK, Clegg AJ.

\section{No. 10}

Payment to healthcare professionals for patient recruitment to trials: systematic review and qualitative study.

By Raftery J, Bryant J, Powell J, Kerr C, Hawker S.

No. 11

Cyclooxygenase-2 selective nonsteroidal anti-inflammatory drugs (etodolac, meloxicam, celecoxib, rofecoxib, etoricoxib, valdecoxib and lumiracoxib) for osteoarthritis and rheumatoid arthritis: a systematic review and economic evaluation.

By Chen Y-F, Jobanputra P, Barton P, Bryan S, Fry-Smith A, Harris G, et al.

\section{No. 12}

The clinical effectiveness and costeffectiveness of central venous catheters treated with anti-infective agents in preventing bloodstream infections: a systematic review and economic evaluation.

By Hockenhull JC, Dwan K, Boland A, Smith G, Bagust A, Dundar Y, et al.

\section{No. 13}

Stepped treatment of older adults on laxatives. The STOOL trial.

By Mihaylov S, Stark C, McColl E, Steen N, Vanoli A, Rubin G, et al.

\section{No. 14}

A randomised controlled trial of cognitive behaviour therapy in adolescents with major depression treated by selective serotonin reuptake inhibitors. The ADAPT trial.

By Goodyer IM, Dubicka B, Wilkinson P, Kelvin R, Roberts C, Byford S, et al.

\section{No. 15}

The use of irinotecan, oxaliplatin and raltitrexed for the treatment of advanced colorectal cancer: systematic review and economic evaluation.

By Hind D, Tappenden P, Tumur I, Eggington E, Sutcliffe P, Ryan A.

\section{No. 16}

Ranibizumab and pegaptanib for the treatment of age-related macular degeneration: a systematic review and economic evaluation.

By Colquitt JL, Jones J, Tan SC, Takeda A, Clegg AJ, Price A.

\section{No. 17}

Systematic review of the clinical effectiveness and cost-effectiveness of 64-slice or higher computed tomography angiography as an alternative to invasive coronary angiography in the investigation of coronary artery disease.

By Mowatt G, Cummins E, Waugh N, Walker S, Cook J, Jia X, et al.

\section{No. 18}

Structural neuroimaging in psychosis: a systematic review and economic evaluation.

By Albon E, Tsourapas A, Frew E, Davenport C, Oyebode F, Bayliss S, et al.

No. 19

Systematic review and economic analysis of the comparative effectiveness of different inhaled corticosteroids and their usage with long-acting beta agonists for the treatment of chronic asthma in adults and children aged 12 years and over.

By Shepherd J, Rogers G, Anderson $\mathrm{R}$, Main C, Thompson-Coon J, Hartwell D, et al.

\section{No. 20}

Systematic review and economic analysis of the comparative effectiveness of different inhaled corticosteroids and their usage with long-acting beta agonists for the treatment of chronic asthma in children under the age of 12 years.

By Main C, Shepherd J, Anderson R, Rogers G, Thompson-Coon J, Liu Z, et al.

\section{No. 21}

Ezetimibe for the treatment of hypercholesterolaemia: a systematic review and economic evaluation.

By Ara R, Tumur I, Pandor A, Duenas A, Williams R, Wilkinson A, et al. 
No. 22

Topical or oral ibuprofen for chronic knee pain in older people. The TOIB study.

By Underwood M, Ashby D, Carnes D, Castelnuovo E, Cross P, Harding G, et al.

No. 23

A prospective randomised comparison of minor surgery in primary and secondary care. The MiSTIC trial.

By George S, Pockney P, Primrose J, Smith H, Little P, Kinley H, et al.

\section{No. 24}

A review and critical appraisal of measures of therapist-patient interactions in mental health settings.

By Cahill J, Barkham M, Hardy G, Gilbody S, Richards D, Bower P, et al.

\section{No. 25}

The clinical effectiveness and costeffectiveness of screening programmes for amblyopia and strabismus in children up to the age of 4-5 years: a systematic review and economic evaluation.

By Carlton J, Karnon J, CzoskiMurray C, Smith KJ, Marr J.

\section{No. 26}

A systematic review of the clinical effectiveness and cost-effectiveness and economic modelling of minimal incision total hip replacement approaches in the management of arthritic disease of the hip.

By de Verteuil R, Imamura M, Zhu S, Glazener C, Fraser C, Munro N, et al.

\section{No. 27}

A preliminary model-based assessment of the cost-utility of a screening programme for early age-related macular degeneration.

By Karnon J, Czoski-Murray C, Smith $\mathrm{K}$, Brand C, Chakravarthy U, Davis S, et al.

\section{No. 28}

Intravenous magnesium sulphate and sotalol for prevention of atrial fibrillation after coronary artery bypass surgery: a systematic review and economic evaluation.

By Shepherd J, Jones J, Frampton GK, Tanajewski L, Turner D, Price A.

\section{No. 29}

Absorbent products for urinary/faecal incontinence: a comparative evaluation of key product categories.

By Fader M, Cottenden A, Getliffe K, Gage H, Clarke-O’Neill S, Jamieson K, et al.

\section{No. 30}

A systematic review of repetitive functional task practice with modelling of resource use, costs and effectiveness.

By French B, Leathley M, Sutton C, McAdam J, Thomas L, Forster A, et al.

\section{No. 31}

The effectiveness and cost-effectivness of minimal access surgery amongst people with gastro-oesophageal reflux disease - a UK collaborative study. The REFLUX trial.

By Grant A, Wileman S, Ramsay C, Bojke L, Epstein D, Sculpher M, et al.

\section{No. 32}

Time to full publication of studies of anti-cancer medicines for breast cancer and the potential for publication bias: a short systematic review.

By Takeda A, Loveman E, Harris P, Hartwell D, Welch K.

\section{No. 33}

Performance of screening tests for child physical abuse in accident and emergency departments.

By Woodman J, Pitt M, Wentz R, Taylor B, Hodes D, Gilbert RE.

\section{No. 34}

Curative catheter ablation in atrial fibrillation and typical atrial flutter: systematic review and economic evaluation.

By Rodgers M, McKenna C, Palmer S, Chambers D, Van Hout S, Golder S, et al.

\section{No. 35}

Systematic review and economic modelling of effectiveness and cost utility of surgical treatments for men with benign prostatic enlargement.

By Lourenco T, Armstrong N, N'Dow J, Nabi G, Deverill M, Pickard R, et al.

\section{No. 36}

Immunoprophylaxis against respiratory syncytial virus (RSV) with palivizumab in children: a systematic review and economic evaluation.

By Wang D, Cummins C, Bayliss S Sandercock J, Burls A.

\section{Volume 13, 2009}

\section{No. 1}

Deferasirox for the treatment of iron overload associated with regular blood transfusions (transfusional haemosiderosis) in patients suffering with chronic anaemia: a systematic review and economic evaluation.

By McLeod C, Fleeman N, Kirkham J, Bagust A, Boland A, Chu P, et al.

\section{No. 2}

Thrombophilia testing in people with venous thromboembolism: systematic review and cost-effectiveness analysis.

By Simpson EL, Stevenson MD, Rawdin A, Papaioannou D.
No. 3

Surgical procedures and non-surgica devices for the management of nonapnoeic snoring: a systematic review of clinical effects and associated treatment costs.

By Main C, Liu Z, Welch K, Weiner G, Quentin Jones S, Stein K.

\section{No. 4}

Continuous positive airway pressure devices for the treatment of obstructive sleep apnoea-hypopnoea syndrome: a systematic review and economic analysis.

By McDaid C, Griffin S, Weatherly H, Durée K, van der Burgt M, van Hout S, Akers J, et al.

\section{No. 5}

Use of classical and novel biomarkers as prognostic risk factors for localised prostate cancer: a systematic review.

By Sutcliffe P, Hummel S, Simpson E, Young T, Rees A, Wilkinson A, et al.

No. 6

The harmful health effects of recreational ecstasy: a systematic review of observational evidence.

By Rogers G, Elston J, Garside R, Roome C, Taylor R, Younger P, et al.

\section{No. 7}

Systematic review of the clinical effectiveness and cost-effectiveness of oesophageal Doppler monitoring in critically ill and high-risk surgical patients.

By Mowatt G, Houston G, Hernández R, de Verteuil R, Fraser C, Cuthbertson $\mathrm{B}$, et al.

No. 8

The use of surrogate outcomes in modelbased cost-effectiveness analyses: a survey of UK Health Technology Assessment reports.

By Taylor RS, Elston J.

No. 9

Controlling Hypertension and

Hypotension Immediately Post Stroke (CHHIPS) - a randomised controlled trial.

By Potter J, Mistri A, Brodie F, Chernova J, Wilson E, Jagger C, et al.

No. 10

Routine antenatal anti-D prophylaxis for RhD-negative women: a systematic review and economic evaluation.

By Pilgrim H, Lloyd-Jones M, Rees A.

\section{No. 11}

Amantadine, oseltamivir and zanamivir for the prophylaxis of influenza (including a review of existing guidance no. 67): a systematic review and economic evaluation.

By Tappenden P, Jackson R, Cooper K, Rees A, Simpson E, Read R, et al. 


\section{No. 12}

Improving the evaluation of therapeutic interventions in multiple sclerosis: the role of new psychometric methods.

By Hobart J, Cano S.

\section{No. 13}

Treatment of severe ankle sprain: a pragmatic randomised controlled trial comparing the clinical effectiveness and cost-effectiveness of three types of mechanical ankle support with tubular bandage. The CAST trial.

By Cooke MW, Marsh JL, Clark M, Nakash R, Jarvis RM, Hutton JL, et al., on behalf of the CAST trial group.

\section{No. 14}

Non-occupational postexposure prophylaxis for HIV: a systematic review.

By Bryant J, Baxter L, Hird S.

\section{No. 15}

Blood glucose self-monitoring in type 2 diabetes: a randomised controlled trial.

By Farmer AJ, Wade AN, French DP, Simon J, Yudkin P, Gray A, et al.

No. 16

How far does screening women for domestic (partner) violence in different health-care settings meet criteria for a screening programme? Systematic reviews of nine UK National Screening Committee criteria.

By Feder G, Ramsay J, Dunne D, Rose M, Arsene C, Norman R, et al.

\section{No. 17}

Spinal cord stimulation for chronic pain of neuropathic or ischaemic origin: systematic review and economic evaluation.

By Simpson EL, Duenas A, Holmes MW, Papaioannou D, Chilcott J.

\section{No. 18}

The role of magnetic resonance imaging in the identification of suspected acoustic neuroma: a systematic review of clinical and cost-effectiveness and natural history.

By Fortnum H, O'Neill C, Taylor R, Lenthall R, Nikolopoulos T, Lightfoot $\mathrm{G}$, et al.

\section{No. 19}

Dipsticks and diagnostic algorithms in urinary tract infection: development and validation, randomised trial, economic analysis, observational cohort and qualitative study.

By Little P, Turner S, Rumsby K, Warner G, Moore M, Lowes JA, et al.

\section{No. 20}

Systematic review of respite care in the frail elderly.

By Shaw C, McNamara R, Abrams K, Cannings-John R, Hood K, Longo M, et al.
No. 21

\section{No. 22} determine the clinical effectiveness

\section{No. 23}

\section{No. 24}

No. 25 patients with abnormal liver function (ALFIE).

No. 26 consent systems for deceased organ

\section{No. 27}

No. 28

A randomised controlled trial to
Neuroleptics in the treatment of aggressive challenging behaviour for people with intellectual disabilities: a randomised controlled trial (NACHBID).

By Tyrer P, Oliver-Africano P, Romeo R, Knapp M, Dickens S, Bouras N, et al.

Randomised controlled trial to and cost-effectiveness of selective serotonin reuptake inhibitors plus supportive care, versus supportive care alone, for mild to moderate depression with somatic symptoms in primary care: the THREAD (THREshold for AntiDepressant response) study.

By Kendrick T, Chatwin J, Dowrick C, Tylee A, Morriss R, Peveler R, et al.

Diagnostic strategies using DNA testing for hereditary haemochromatosis in at-risk populations: a systematic review and economic evaluation.

By Bryant J, Cooper K, Picot J, Clege A, Roderick P, Rosenberg W, et al.

Enhanced external counterpulsation for the treatment of stable angina and heart failure: a systematic review and economic analysis.

By McKenna C, McDaid C, Suekarran S, Hawkins N, Claxton K, Light K, et al.

Development of a decision support tool for primary care management of tests without clinically apparent liver disease: a record-linkage population cohort study and decision analysis

By Donnan PT, McLernon D, Dillon JF, Ryder S, Roderick P, Sullivan F, et al.

A systematic review of presumed donation.

By Rithalia A, McDaid C, Suekarran S, Norman G, Myers L, Sowden A.

Paracetamol and ibuprofen for the treatment of fever in children: the PITCH randomised controlled trial.

By Hay AD, Redmond NM, Costelloe C, Montgomery AA, Fletcher M, Hollinghurst S, et al. compare minimally invasive glucose monitoring devices with conventional monitoring in the management of insulin-treated diabetes mellitus (MITRE).

By Newman SP, Cooke D, Casbard A

\section{No. 29}

Sensitivity analysis in economic evaluation: an audit of NICE current practice and a review of its use and value in decision-making.

By Andronis L, Barton P, Bryan S.

\section{Suppl. 1}

Trastuzumab for the treatment of primary breast cancer in HER2-positive women: a single technology appraisal.

By Ward S, Pilgrim H, Hind D.

Docetaxel for the adjuvant treatment of early node-positive breast cancer: a single technology appraisal.

By Chilcott J, Lloyd Jones M, Wilkinson A.

The use of paclitaxel in the management of early stage breast cancer.

By Griffin S, Dunn G, Palmer S, Macfarlane K, Brent S, Dyker A, et al.

Rituximab for the first-line treatment of stage III/IV follicular non-Hodgkin's lymphoma.

By Dundar Y, Bagust A, Hounsome J, McLeod C, Boland A, Davis H, et al.

Bortezomib for the treatment of multiple myeloma patients.

By Green C, Bryant J, Takeda A, Cooper K, Clegg A, Smith A, et al.

Fludarabine phosphate for the firstline treatment of chronic lymphocytic leukaemia.

By Walker S, Palmer S, Erhorn S, Brent S, Dyker A, Ferrie L, et al.

Erlotinib for the treatment of relapsed non-small cell lung cancer

By McLeod C, Bagust A, Boland A, Hockenhull J, Dundar Y, Proudlove C, et al.

Cetuximab plus radiotherapy for the treatment of locally advanced squamou cell carcinoma of the head and neck.

By Griffin S, Walker S, Sculpher M, White S, Erhorn S, Brent S, et al.

Infliximab for the treatment of adults with psoriasis.

By Loveman E, Turner D, Hartwell D, Cooper K, Clegg A.

No. 30

Psychological interventions for postnatal depression: cluster randomised trial and economic evaluation. The PoNDER trial.

By Morrell CJ, Warner R, Slade P, Dixon S, Walters S, Paley G, et al.

No. 31

The effect of different treatment durations of clopidogrel in patients with non-ST-segment elevation acute coronary syndromes: a systematic review and value of information analysis.

By Rogowski R, Burch J, Palmer S, Walker S, Meredith S, Nunn A, et al. 


\section{No. 32}

Systematic review and individual patient data meta-analysis of diagnosis of heart failure, with modelling of implications of different diagnostic strategies in primary care.

By Mant J, Doust J, Roalfe A, Barton P, Cowie MR, Glasziou P, et al.

\section{No. 33}

A multicentre randomised controlled trial of the use of continuous positive airway pressure and non-invasive positive pressure ventilation in the early treatment of patients presenting to the emergency department with severe acute cardiogenic pulmonary oedema: the 3CPO trial.

By Gray AJ, Goodacre S, Newby DE, Masson MA, Sampson F, Dixon $\mathrm{S}$, et al., on behalf of the 3CPO study investigators.

\section{No. 34}

Early high-dose lipid-lowering therapy to avoid cardiac events: a systematic review and economic evaluation.

By Ara R, Pandor A, Stevens J, Rees A, Rafia $\mathrm{R}$

\section{No. 35}

Adefovir dipivoxil and pegylated interferon alpha for the treatment of chronic hepatitis B: an updated systematic review and economic evaluation.

By Jones J, Shepherd J, Baxter L, Gospodarevskaya E, Hartwell D, Harris $\mathrm{P}$, et al.

\section{No. 36}

Methods to identify postnatal depression in primary care: an integrated evidence synthesis and value of information analysis.

By Hewitt CE, Gilbody SM, Brealey S, Paulden M, Palmer S, Mann R, et al.

\section{No. 37}

A double-blind randomised placebocontrolled trial of topical intranasal corticosteroids in 4- to 11-year-old children with persistent bilateral otitis media with effusion in primary care.

By Williamson I, Benge S, Barton S, Petrou S, Letley L, Fasey N, et al.

\section{No. 38}

The effectiveness and cost-effectiveness of methods of storing donated kidneys from deceased donors: a systematic review and economic model.

By Bond M, Pitt M, Akoh J, Moxham T, Hoyle M, Anderson R.

\section{No. 39}

Rehabilitation of older patients: day hospital compared with rehabilitation at home. A randomised controlled trial.

By Parker SG, Oliver P, Pennington M, Bond J, Jagger C, Enderby PM, et al.

\section{No. 40}

Breastfeeding promotion for infants in neonatal units: a systematic review and economic analysis.

By Renfrew MJ, Craig D, Dyson L, McCormick F, Rice S, King SE, et al.

\section{No. 41}

The clinical effectiveness and costeffectiveness of bariatric (weight loss) surgery for obesity: a systematic review and economic evaluation.

By Picot J, Jones J, Colquitt JL, Gospodarevskaya E, Loveman E, Baxter $\mathrm{L}$, et al.

\section{No. 42}

Rapid testing for group B streptococcus during labour: a test accuracy study with evaluation of acceptability and costeffectiveness.

By Daniels J, Gray J, Pattison H, Roberts T, Edwards E, Milner P, et al.

\section{No. 43}

Screening to prevent spontaneous preterm birth: systematic reviews of accuracy and effectiveness literature with economic modelling.

By Honest H, Forbes CA, Durée KH, Norman G, Duffy SB, Tsourapas A, et al

\section{No. 44}

The effectiveness and cost-effectiveness of cochlear implants for severe to profound deafness in children and adults: a systematic review and economic model.

By Bond M, Mealing S, Anderson R Elston J, Weiner G, Taylor RS, et al.

\section{Suppl. 2}

Gemcitabine for the treatment of metastatic breast cancer.

By Jones J, Takeda A, Tan SC, Cooper $\mathrm{K}$, Loveman E, Clegg A.

Varenicline in the management of smoking cessation: a single technology appraisal.

By Hind D, Tappenden P, Peters J, Kenjegalieva K.

Alteplase for the treatment of acute ischaemic stroke: a single technology appraisal.

By Lloyd Jones M, Holmes M.

Rituximab for the treatment of rheumatoid arthritis.

By Bagust A, Boland A, Hockenhull J, Fleeman N, Greenhalgh J, Dundar Y, et al.

Omalizumab for the treatment of severe persistent allergic asthma.

By Jones J, Shepherd J, Hartwell D, Harris P, Cooper K, Takeda A, et al.

Rituximab for the treatment of relapsed or refractory stage III or IV follicular non-Hodgkin's lymphoma.

By Boland A, Bagust A, Hockenhull J, Davis H, Chu P, Dickson R.
Adalimumab for the treatment of psoriasis.

By Turner D, Picot J, Cooper K, Loveman E.

Dabigatran etexilate for the prevention of venous thromboembolism in patients undergoing elective hip and knee

surgery: a single technology appraisal. By Holmes M, Carroll C,

Papaioannou D.

Romiplostim for the treatment of chronic immune or idiopathic thrombocytopenic purpura: a single technology appraisal.

By Mowatt G, Boachie C, Crowther M, Fraser C, Hernández R, Jia X, et al.

Sunitinib for the treatment of gastrointestinal stromal tumours: a critique of the submission from Pfizer.

By Bond M, Hoyle M, Moxham T, Napier M, Anderson R.

No. 45

Vitamin K to prevent fractures in older women: systematic review and economic evaluation.

By Stevenson M, Lloyd-Jones M, Papaioannou D.

No. 46

The effects of biofeedback for the treatment of essential hypertension: a systematic review.

By Greenhalgh J, Dickson R, Dundar Y.

No. 47

A randomised controlled trial of the use of aciclovir and/or prednisolone for the early treatment of Bell's palsy: the BELLS study.

By Sullivan FM, Swan IRC, Donnan PT, Morrison JM, Smith BH, McKinstry $\mathrm{B}$, et al.

Suppl. 3

Lapatinib for the treatment of HER2overexpressing breast cancer.

By Jones J, Takeda A, Picot J, von

Keyserlingk C, Clegg A.

Infliximab for the treatment of ulcerative colitis.

By Hyde C, Bryan S, Juarez-Garcia A, Andronis L, Fry-Smith A.

Rimonabant for the treatment of overweight and obese people.

By Burch J, McKenna C, Palmer S, Norman G, Glanville J, Sculpher M, et al.

Telbivudine for the treatment of chronic hepatitis B infection.

By Hartwell D, Jones J, Harris P, Cooper K

Entecavir for the treatment of chronic hepatitis B infection.

By Shepherd J, Gospodarevskaya E, Frampton G, Cooper K.

Febuxostat for the treatment of hyperuricaemia in people with gout: a single technology appraisal.

By Stevenson M, Pandor A. 
Rivaroxaban for the prevention of venous thromboembolism: a single technology appraisal.

By Stevenson M, Scope A, Holmes M, Rees A, Kaltenthaler E.

Cetuximab for the treatment of recurrent and/or metastatic squamous cell carcinoma of the head and neck.

By Greenhalgh J, Bagust A, Boland A, Fleeman N, McLeod C, Dundar Y, et al.

Mifamurtide for the treatment of osteosarcoma: a single technology appraisal.

By Pandor A, Fitzgerald P, Stevenson M, Papaioannou D.

Ustekinumab for the treatment of moderate to severe psoriasis.

By Gospodarevskaya E, Picot J, Cooper K, Loveman E, Takeda A.

\section{No. 48}

Endovascular stents for abdominal aortic aneurysms: a systematic review and economic model.

By Chambers D, Epstein D, Walker S, Fayter D, Paton F, Wright K, et al.

\section{No. 49}

Clinical and cost-effectiveness of epoprostenol, iloprost, bosentan, sitaxentan and sildenafil for pulmonary arterial hypertension within their licensed indications: a systematic review and economic evaluation.

By Chen Y-F, Jowett S, Barton P, Malottki K, Hyde C, Gibbs JSR, et al.

\section{No. 50}

Cessation of attention deficit hyperactivity disorder drugs in the young (CADDY) - a pharmacoepidemiological and qualitative study.

By Wong ICK, Asherson P, Bilbow A, Clifford S, Coghill D, DeSoysa R, et al.

No. 51

ARTISTIC: a randomised trial of human papillomavirus (HPV) testing in primary cervical screening.

By Kitchener HC, Almonte M, Gilham C, Dowie R, Stoykova B, Sargent A, et al.

\section{No. 52}

The clinical effectiveness of glucosamine and chondroitin supplements in slowing or arresting progression of osteoarthritis of the knee: a systematic review and economic evaluation.

By Black C, Clar C, Henderson R, MacEachern C, McNamee P, Quayyum $\mathrm{Z}$, et al.

\section{No. 53}

Randomised preference trial of medical versus surgical termination of pregnancy less than 14 weeks' gestation (TOPS).

By Robson SC, Kelly T, Howel D,

Deverill M, Hewison J, Lie MLS, et al.
No. 54

Randomised controlled trial of the use of three dressing preparations in the management of chronic ulceration of the foot in diabetes.

By Jeffcoate WJ, Price PE, Phillips CJ, Game FL, Mudge E, Davies S, et al.

\section{No. 55}

VenUS II: a randomised controlled trial of larval therapy in the management of leg ulcers.

By Dumville JC, Worthy G, Soares MO, Bland JM, Cullum N, Dowson C, et al.

\section{No. 56}

A prospective randomised controlled trial and economic modelling of antimicrobial silver dressings versus non-adherent control dressings for venous leg ulcers: the VULCAN trial.

By Michaels JA, Campbell WB,

King BM, MacIntyre J, Palfreyman SJ, Shackley $\mathrm{P}$, et al.

No. 57

Communication of carrier status information following universal newborn screening for sickle cell disorders and cystic fibrosis: qualitative study of experience and practice.

By Kai J, Ulph F, Cullinan T, Qureshi N

\section{No. 58}

Antiviral drugs for the treatment of influenza: a systematic review and economic evaluation.

By Burch J, Paulden M, Conti S, Stock C, Corbett M, Welton NJ, et al.

\section{No. 59}

Development of a toolkit and glossary to aid in the adaptation of health technology assessment (HTA) reports for use in different contexts.

By Chase D, Rosten C, Turner S, Hicks N, Milne R.

\section{No. 60}

Colour vision testing for diabetic retinopathy: a systematic review of diagnostic accuracy and economic evaluation.

By Rodgers M, Hodges R, Hawkins J, Hollingworth W, Duffy S, McKibbin $\mathrm{M}$, et al.

\section{No. 61}

Systematic review of the effectiveness and cost-effectiveness of weight management schemes for the under fives: a short report

By Bond M, Wyatt K, Lloyd J, Welch $\mathrm{K}$, Taylor R.

\section{No. 62}

Are adverse effects incorporated in economic models? An initial review of current practice.

By Craig D, McDaid C, Fonseca T, Stock C, Duffy S, Woolacott N.

\section{Volume 14, 2010}

No. 1

Multicentre randomised controlled trial examining the cost-effectiveness of contrast-enhanced high field magnetic resonance imaging in women with primary breast cancer scheduled for wide local excision (COMICE).

By Turnbull LW, Brown SR, Olivier C, Harvey I, Brown J, Drew P, et al.

\section{No. 2}

Bevacizumab, sorafenib tosylate, sunitinib and temsirolimus for renal cell carcinoma: a systematic review and economic evaluation.

By Thompson Coon J, Hoyle M, Green C, Liu Z, Welch K, Moxham T, et al.

\section{No. 3}

The clinical effectiveness and costeffectiveness of testing for cytochrome $\mathrm{P} 450$ polymorphisms in patients with schizophrenia treated with antipsychotics: a systematic review and economic evaluation.

By Fleeman N, McLeod C, Bagust A, Beale S, Boland A, Dundar Y, et al.

\section{No. 4}

Systematic review of the clinical effectiveness and cost-effectiveness of photodynamic diagnosis and urine biomarkers (FISH, ImmunoCyt, NMP22) and cytology for the detection and follow-up of bladder cancer.

By Mowatt G, Zhu S, Kilonzo M, Boachie C, Fraser C, Griffiths TRL, et al.

\section{No. 5}

Effectiveness and cost-effectiveness of arthroscopic lavage in the treatment of osteoarthritis of the knee: a mixed methods study of the feasibility of conducting a surgical placebo-controlled trial (the KORAL study).

By Campbell MK, Skea ZC, Sutherland AG, Cuthbertson BH, Entwistle VA, McDonald AM, et al.

No. 6

A randomised $2 \times 2$ trial of community versus hospital pulmonary rehabilitation for chronic obstructive pulmonary disease followed by telephone or conventional follow-up.

By Waterhouse JC, Walters SJ, Oluboyede Y, Lawson RA.

No. 7

The effectiveness and cost-effectiveness of behavioural interventions for the prevention of sexually transmitted infections in young people aged 13-19: a systematic review and economic evaluation.

By Shepherd J, Kavanagh J, Picot J, Cooper K, Harden A, Barnett-Page E, et al. 
No. 8

Dissemination and publication of research findings: an updated review of related biases.

By Song F, Parekh S, Hooper L, Loke YK, Ryder J, Sutton AJ, et al.

\section{No. 9}

The effectiveness and cost-effectiveness of biomarkers for the prioritisation of patients awaiting coronary revascularisation: a systematic review and decision model.

By Hemingway H, Henriksson M, Chen R, Damant J, Fitzpatrick N, Abrams K, et al.

\section{No. 10}

Comparison of case note review methods for evaluating quality and safety in health care.

By Hutchinson A, Coster JE, Cooper KL, McIntosh A, Walters SJ, Bath PA, et al.

\section{No. 11}

Clinical effectiveness and costeffectiveness of continuous subcutaneous insulin infusion for diabetes: systematic review and economic evaluation.

By Cummins E, Royle P, Snaith A, Greene A, Robertson L, McIntyre L, et al.

\section{No. 12}

Self-monitoring of blood glucose in type 2 diabetes: systematic review.

By Clar C, Barnard K, Cummins E, Royle P, Waugh N.

\section{No. 13}

North of England and Scotland Study of Tonsillectomy and Adeno-tonsillectomy in Children (NESSTAC): a pragmatic randomised controlled trial with a parallel non-randomised preference study.

By Lock C, Wilson J, Steen N, Eccles M, Mason H, Carrie S, et al.

\section{No. 14}

Multicentre randomised controlled trial of the clinical and cost-effectiveness of a bypass-surgery-first versus a balloonangioplasty-first revascularisation strategy for severe limb ischaemia due to infrainguinal disease. The Bypass versus Angioplasty in Severe Ischaemia of the Leg (BASIL) trial.

By Bradbury AW, Adam DJ, Bell J, Forbes JF, Fowkes FGR, Gillespie I, et al.

\section{No. 15}

A randomised controlled multicentre trial of treatments for adolescent anorexia nervosa including assessment of cost-effectiveness and patient acceptability - the TOuCAN trial.

By Gowers SG, Clark AF, Roberts C, Byford S, Barrett B, Griffiths A, et al.

\section{No. 16}

Randomised controlled trials for policy interventions: a review of reviews and meta-regression.

By Oliver S, Bagnall AM, Thomas J, Shepherd J, Sowden A, White I, et al.

\section{No. 17}

Paracetamol and selective and non-selective non-steroidal antiinflammatory drugs (NSAIDs) for the reduction of morphine-related side effects after major surgery: a systematic review.

By McDaid C, Maund E, Rice S, Wright K, Jenkins B, Woolacott N.

\section{No. 18}

A systematic review of outcome measures used in forensic mental health research with consensus panel opinion.

By Fitzpatrick R, Chambers J, Burns T, Doll H, Fazel S, Jenkinson C, et al.

\section{No. 19}

The clinical effectiveness and costeffectiveness of topotecan for small cell lung cancer: a systematic review and economic evaluation.

By Loveman E, Jones J, Hartwell D, Bird A, Harris P, Welch K, et al.

\section{No. 20}

Antenatal screening for

haemoglobinopathies in primary care: a cohort study and cluster randomised trial to inform a simulation model. The Screening for Haemoglobinopathies in First Trimester (SHIFT) trial.

By Dormandy E, Bryan S, Gulliford MC, Roberts T, Ades T, Calnan M, et al.

\section{No. 21}

Early referral strategies for management of people with markers of renal disease: a systematic review of the evidence of clinical effectiveness, costeffectiveness and economic analysis.

By Black C, Sharma P, Scotland G,

McCullough K, McGurn D, Robertson $\mathrm{L}$, et al.

\section{No. 22}

A randomised controlled trial of cognitive behaviour therapy and motivational interviewing for people with Type 1 diabetes mellitus with persistent sub-optimal glycaemic control: A Diabetes and Psychological Therapies (ADaPT) study.

By Ismail K, Maissi E, Thomas S, Chalder T, Schmidt U, Bartlett J, et al.

\section{No. 23}

A randomised controlled equivalence trial to determine the effectiveness and cost-utility of manual chest physiotherapy techniques in the management of exacerbations of chronic obstructive pulmonary disease (MATREX).

By Cross J, Elender F, Barton G Clark A, Shepstone L, Blyth A, et al.

\section{No. 24}

A systematic review and economic evaluation of the clinical effectiveness and cost-effectiveness of aldosterone antagonists for postmyocardial infarction heart failure.

By McKenna C, Burch J, Suekarran S, Walker S, Bakhai A, Witte K, et al.

\section{No. 25}

Avoiding and identifying errors in health technology assessment models: qualitative study and methodological review.

By Chilcott JB, Tappenden P, Rawdin A, Johnson M, Kaltenthaler E, Paisley S, et al.

\section{No. 26}

BoTULS: a multicentre randomised controlled trial to evaluate the clinical effectiveness and cost-effectiveness of treating upper limb spasticity due to stroke with botulinum toxin type A.

By Shaw L, Rodgers H, Price C, van Wijck F, Shackley P, Steen N, et al., on behalf of the BoTULS investigators.

No. 27

Weighting and valuing quality-adjusted life-years using stated preference methods: preliminary results from the Social Value of a QALY Project.

By Baker R, Bateman I, Donaldson C, Jones-Lee M, Lancsar E, Loomes G, et al.

\section{Suppl. 1}

Cetuximab for the first-line treatment of metastatic colorectal cancer.

By Meads C, Round J, Tubeuf S, Moore D, Pennant M, Bayliss S.

Infliximab for the treatment of acute exacerbations of ulcerative colitis.

By Bryan S, Andronis L, Hyde C, Connock M, Fry-Smith A, Wang D.

Sorafenib for the treatment of advanced hepatocellular carcinoma.

By Connock M, Round J, Bayliss S, Tubeuf S, Greenheld W, Moore D.

Tenofovir disoproxil fumarate for the treatment of chronic hepatitis B infection.

By Jones J, Colquitt J, Shepherd J, Harris P, Cooper K.

Prasugrel for the treatment of acute coronary artery syndromes with percutaneous coronary intervention.

By Greenhalgh J, Bagust A, Boland A, Saborido CM, Fleeman N, McLeod C, et al.

Alitretinoin for the treatment of severe chronic hand eczema.

By Paulden M, Rodgers M, Griffin S, Slack R, Duffy S, Ingram JR, et al.

Pemetrexed for the first-line treatment of locally advanced or metastatic nonsmall cell lung cancer.

By Fleeman N, Bagust A, McLeod C, Greenhalgh J, Boland A, Dundar Y, et al. 
Topotecan for the treatment of recurrent and stage IVB carcinoma of the cervix.

By Paton F, Paulden M, Saramago P, Manca A, Misso K, Palmer S, et al.

Trabectedin for the treatment of advanced metastatic soft tissue sarcoma.

By Simpson EL, Rafia R, Stevenson MD, Papaioannou D.

Azacitidine for the treatment of myelodysplastic syndrome, chronic myelomonocytic leukaemia and acute myeloid leukaemia.

By Edlin R, Connock M, Tubeuf S Round J, Fry-Smith A, Hyde C, et al.

No. 28

The safety and effectiveness of different methods of earwax removal: a systematic review and economic evaluation.

By Clegg AJ, Loveman E, Gospodarevskaya E, Harris P, Bird A, Bryant J, et al.

\section{No. 29}

Systematic review of the clinical effectiveness and cost-effectiveness of rapid point-of-care tests for the detection of genital chlamydia infection in women and men.

By Hislop J, Quayyum Z, Flett G, Boachie C, Fraser C, Mowatt G.

\section{No. 30}

School-linked sexual health services for young people (SSHYP): a survey and systematic review concerning current models, effectiveness, cost-effectiveness and research opportunities.

By Owen J, Carroll C, Cooke J, Formby E, Hayter M, Hirst J, et al.

\section{No. 31}

Systematic review and cost-effectiveness evaluation of 'pill-in-the-pocket' strategy for paroxysmal atrial fibrillation compared to episodic in-hospital treatment or continuous antiarrhythmic drug therapy.

By Martin Saborido C, Hockenhull J, Bagust A, Boland A, Dickson R, Todd D.

\section{No. 32}

Chemoprevention of colorectal cancer: systematic review and economic evaluation.

By Cooper K, Squires H, Carroll C, Papaioannou D, Booth A, Logan RF, et al.
No. 33

Cross-trimester repeated measures testing for Down's syndrome screening: an assessment.

By Wright D, Bradbury I, Malone F, D'Alton M, Summers A, Huang T, et al.

No. 34

Exploring the needs, concerns and behaviours of people with existing respiratory conditions in relation to the H1N1 'swine influenza' pandemic: a multicentre survey and qualitative study.

By Caress A-L, Duxbury P, Woodcock A, Luker KA, Ward D, Campbell M, et al.

Influenza $\mathrm{A} / \mathrm{H} 1 \mathrm{~N} 1 \mathrm{v}$ in pregnancy: an investigation of the characteristics and management of affected women and the relationship to pregnancy outcomes for mother and infant.

By Yates L, Pierce M, Stephens S, Mill AC, Spark P, Kurinczuk JJ, et al.

The impact of communications about swine flu (influenza A H1N1v) on public responses to the outbreak: results from 36 national telephone surveys in the UK.

By Rubin GJ, Potts HWW, Michie S.

The impact of illness and the impact of school closure on social contact patterns.

By Eames KTD, Tilston NL, White PJ, Adams E, Edmunds WJ.

Vaccine effectiveness in pandemic influenza - primary care reporting (VIPER): an observational study to assess the effectiveness of the pandemic influenza $\mathrm{A}(\mathrm{H} 1 \mathrm{~N} 1) \mathrm{v}$ vaccine.

By Simpson CR, Ritchie LD, Robertson C, Sheikh A, McMenamin J.

Physical interventions to interrupt or reduce the spread of respiratory viruses: a Cochrane review.

By Jefferson T, Del Mar C, Dooley L, Ferroni E, Al-Ansary LA, Bawazeer GA, et al.

No. 35

Randomised controlled trial and parallel economic evaluation of conventional ventilatory support versus extracorporeal membrane oxygenation for severe adult respiratory failure (CESAR).

By Peek GJ, Elbourne D, Mugford M, Tiruvoipati R, Wilson A, Allen E, et al.

\section{No. 36}

Newer agents for blood glucose control in type 2 diabetes: systematic review and economic evaluation.

By Waugh N, Cummins E, Royle P, Clar C, Marien M, Richter B, et al.

No. 37

Barrett's oesophagus and cancers of the biliary tract, brain, head and neck, lung, oesophagus and skin.

By Fayter D, Corbett M, Heirs M, Fox D, Eastwood A.

\section{No. 38}

Towards single embryo transfer?

Modelling clinical outcomes of potential treatment choices using multiple data sources: predictive models and patient perspectives.

By Roberts SA, McGowan L, Hirst

WM, Brison DR, Vail A, Lieberman BA.

No. 39

Sugammadex for the reversal of muscle relaxation in general anaesthesia: a systematic review and economic assessment.

By Chambers D, Paulden M, Paton F, Heirs M, Duffy S, Craig D, et al.

No. 40

Systematic review and economic modelling of the effectiveness and costeffectiveness of non-surgical treatments for women with stress urinary incontinence.

By Imamura M, Abrams P, Bain C, Buckley B, Cardozo L, Cody J, et al. 


\section{Health Technology Assessment programme}

\author{
Director, \\ Professor Tom Walley, \\ Director, NIHR HTA \\ programme, Professor of \\ Clinical Pharmacology, \\ University of Liverpoo
}

\author{
Deputy Director, \\ Professor Jon Nicholl, \\ Director, Medical Care Research \\ Unit, University of Sheffield
}

Members

\section{Chair,}

Professor Tom Walley,

Director, NIHR HTA

programme, Professor of

Clinical Pharmacology,

University of Liverpool

\section{Deputy Chair,}

Professor Jon Nicholl,

Director, Medical Care Research

Unit, University of Sheffield

Dr Bob Coates,

Consultant Advisor, NETSCC,

HTA

\section{Prioritisation Strategy Group}

\author{
Dr Andrew Cook, \\ Consultant Advisor, NETSCC, \\ HTA \\ Dr Peter Davidson, \\ Director of NETSCC, Health \\ Technology Assessment \\ Professor Robin E Ferner, \\ Consultant Physician and \\ Director, West Midlands Centre \\ for Adverse Drug Reactions, \\ City Hospital NHS Trust, \\ Birmingham
}

Professor Paul Glasziou,

Professor of Evidence-Based

Medicine, University of Oxford

Dr Nick Hicks,

Consultant Adviser, NETSCC,

HTA

Dr Edmund Jessop,

Medical Adviser, National

Specialist, National

Commissioning Group (NCG),

Department of Health, London
Ms Lynn Kerridge,

Chief Executive Officer,

NETSCC and NETSCC, HTA

Dr Ruairidh Milne,

Director of NETSCC External

Relations

Ms Kay Pattison,

Senior NIHR Programme Manager, Department of

Health

Ms Pamela Young,

Specialist Programme Manager, NETSCC, HTA

\section{HTA Commissioning Board}

\section{Members}

Programme Director,

Professor Tom Walley,

Director, NIHR HTA

programme, Professor of

Clinical Pharmacology,

University of Liverpool

\section{Chairs,}

Professor Sallie Lamb,

Director, Warwick Clinical Trials

Unit

Professor Hywel Williams,

Director, Nottingham Clinical

Trials Unit

\section{Deputy Chair,}

Dr Andrew Farmer,

Senior Lecturer in General

Practice, Department of

Primary Health Care,

University of Oxford

Professor Ann Ashburn,

Professor of Rehabilitation

and Head of Research,

Southampton General Hospital
Professor Deborah Ashby, Professor of Medical Statistics, Queen Mary, University of London

Professor John Cairns, Professor of Health Economics, London School of Hygiene and Tropical Medicine

Professor Peter Croft, Director of Primary Care

Sciences Research Centre, Keele University

Professor Nicky Cullum, Director of Centre for EvidenceBased Nursing, University of York

Professor Jenny Donovan, Professor of Social Medicine, University of Bristol

Professor Steve Halligan, Professor of Gastrointestinal Radiology, University College Hospital, London
Professor Freddie Hamdy, Professor of Urology,

University of Sheffield

Professor Allan House, Professor of Liaison Psychiatry, University of Leeds

Dr Martin J Landray, Reader in Epidemiology, Honorary Consultant Physician, Clinical Trial Service Unit,

University of Oxford

Professor Stuart Logan, Director of Health \& Social Care Research, The Peninsula Medical School, Universities of Exeter and Plymouth

Dr Rafael Perera,

Lecturer in Medical Statisitics, Department of Primary Health Care, University of Oxford
Professor Ian Roberts, Professor of Epidemiology \& Public Health, London School of Hygiene and Tropical Medicine

Professor Mark Sculpher, Professor of Health Economics, University of York

Professor Helen Smith, Professor of Primary Care, University of Brighton

Professor Kate Thomas, Professor of Complementary \& Alternative Medicine Research, University of Leeds

Professor David John Torgerson,

Director of York Trials Unit, University of York

\section{Observers}

Ms Kay Pattison,

Section Head, NHS R\&D

Programme, Department of Health
Dr Morven Roberts,

Clinical Trials Manager,

Medical Research Council 


\section{Diagnostic Technologies and Screening Panel}

Members

Chair,
Professor Paul Glasziou,
Professor of Evidence-Based
Medicine, University of Oxford
Deputy Chair,
Dr David Elliman,
Consultant Paediatrician and
Honorary Senior Lecturer,
Great Ormond Street Hospital,
London
Professor Judith E Adams,
Consultant Radiologist,
Manchester Royal Infirmary,
Central Manchester \&
Manchester Children's
University Hospitals NHS
Trust, and Professor of
Diagnostic Radiology, Imaging
Science and Biomedical
Engineering, Cancer \&
Imaging Sciences, University of
Manchester

Dr Dianne Baralle,

Consultant \& Senior Lecturer

in Clinical Genetics, Human

Genetics Division \& Wessex

Clinical Genetics Service,

Southampton, University of

Southampton

Dr Stephanie Dancer,

Consultant Microbiologist,

Hairmyres Hospital, East

Kilbride

Dr Ron Gray,

Consultant, National Perinatal Epidemiology Unit, Institute of Health Sciences, University of Oxford

Professor Paul D Griffiths, Professor of Radiology, Academic Unit of Radiology, University of Sheffield

Mr Martin Hooper,

Service User Representative
Professor Anthony Robert Kendrick,

Professor of Primary

Medical Care, University of Southampton

Dr Susanne M Ludgate, Director, Medical Devices Agency, London

Dr Anne Mackie, Director of Programmes, UK National Screening Committee

Dr David Mathew Service User Representative

Dr Michael Millar, Lead Consultant in Microbiology, Department of Pathology \& Microbiology, Barts and The London NHS Trust, Royal London Hospital

Mr Stephen Pilling, Director, Centre for Outcomes, Research \& Effectiveness, University College London
Mrs Una Rennard, Service User Representative

Ms Jane Smith, Consultant Ultrasound Practitioner, Ultrasound Department, Leeds Teaching Hospital NHS Trust, Leeds

Dr W Stuart A Smellie, Consultant, Bishop Auckland General Hospital

Professor Lindsay Wilson Turnbull,

Scientific Director of the Centre for Magnetic Resonance Investigations and YCR Professor of Radiology, Hull Royal Infirmary

Dr Alan J Williams, Consultant in Genera Medicine, Department of Thoracic Medicine, The Royal Bournemouth Hospital

Honorary Senior Lecturer,

University of Birmingham and

Consultant Urogynaecologist

and Obstetrician, City Hospital

\section{Observers}

Dr Tim Elliott,

Team Leader, Cancer

Screening, Department of

Health
Dr Catherine Moody,

Programme Manager,

Neuroscience and Mental

Health Board
Dr Ursula Wells,

Principal Research Officer

Department of Health

\section{Disease Prevention Panel}

Members

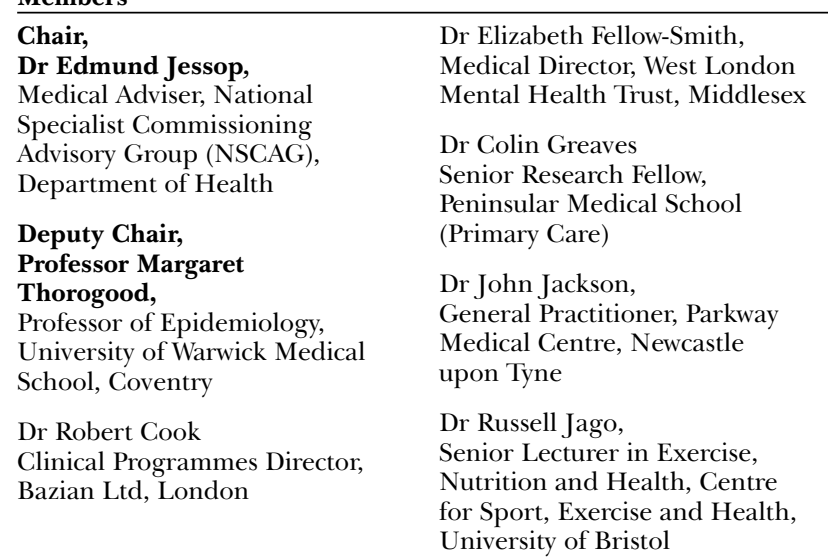

Dr Chris McCall,

General Practitioner, The

Hadleigh Practice, Corfe

Mullen, Dorset

Miss Nicky Mullany, Service User Representative

Dr Julie Mytton,

Locum Consultant in Public

Health Medicine, Bristol

Primary Care Trust

Professor Irwin Nazareth, Professor of Primary Care and Director, Department of Primary Care and Population Sciences, University College

London
Professor Ian Roberts,

Professor of Epidemiology and Public Health, London School of Hygiene \& Tropical Medicine

Professor Carol Tannahill, Glasgow Centre for Population Health

Mrs Jean Thurston, Service User Representative

Professor David Weller Head, School of Clinical Science and Community Health, University of Edinburgh

\section{Observers}

Ms Christine McGuire, Research \& Development, Department of Health
Ms Kay Pattison

Senior NIHR Programme

Manager, Department of

Health
Dr Caroline Stone,

Programme Manager, Medical Research Council 


\section{External Devices and Physical Therapies Panel}

Members

\section{Chair, \\ Dr John Pounsford, \\ Consultant Physician North \\ Bristol NHS Trust, Bristol \\ Deputy Chair, \\ Professor E Andrea Nelson,}

Reader in Wound Healing and

Director of Research, University

of Leeds, Leeds

Professor Bipin Bhakta

Charterhouse Professor in

Rehabilitation Medicine,

University of Leeds, Leeds

Mrs Penny Calder

Service User Representative

Professor Paul Carding,

Professor of Voice Pathology,

Newcastle Hospital NHS Trust,

Newcastle
Dr Dawn Carnes,

Senior Research Fellow, Barts

and the London School of

Medicine and Dentistry,

London

Dr Emma Clark,

Clinician Scientist Fellow

\& Cons. Rheumatologist,

University of Bristol, Bristol

Mrs Anthea De Barton-Watson, Service User Representative

Professor Christopher Griffiths, Professor of Primary Care,

Barts and the London School

of Medicine and Dentistry,

London

Dr Shaheen Hamdy,

Clinical Senior Lecturer

and Consultant Physician,

University of Manchester,

Manchester
Dr Peter Martin,

Consultant Neurologist,

Addenbrooke's Hospital,

Cambridge

Dr Lorraine Pinnigton,

Associate Professor in

Rehabilitation, University of

Nottingham, Nottingham

Dr Kate Radford,

Division of Rehabilitation and

Ageing, School of Community

Health Sciences. University of

Nottingham, Nottingham

Mr Jim Reece,

Service User Representative

Professor Maria Stokes,

Professor of

Neuromusculoskeletal

Rehabilitation, University of

Southampton, Southampton
Dr Pippa Tyrrell,

Stroke Medicine, Senior

Lecturer/Consultant Stroke

Physician, Salford Royal

Foundation Hospitals' Trust,

Salford

Dr Sarah Tyson,

Senior Research Fellow \&

Associate Head of School,

University of Salford, Salford

Dr Nefyn Williams,

Clinical Senior Lecturer, Cardiff

University, Cardiff

Dr Morven Roberts,

Dr Ursula Wells

Ms Kay Pattison
Senior NIHR Programme

Clinical Trials Manager, MRC,
Lr Morven Roberts,

Manager, Department of

London
PRP, DH, London
Primary Care, Department of

Health, London
Health

\section{Interventional Procedures Panel}

Members

Chair,

Consultant Surgeon \&

Honorary Clinical Lecturer,

University of Sheffield

Mr David P Britt,

Service User Representative,

Cheshire

Mr Sankaran

ChandraSekharan,

Consultant Surgeon, Colchester

Hospital University NHS

Foundation Trust

Professor Nicholas Clarke,

Consultant Orthopaedic

Surgeon, Southampton

University Hospitals NHS Trust
Mr Seamus Eckford,

Consultant in Obstetrics \&

Gynaecology, North Devon

District Hospital

Professor David Taggart,

Consultant Cardiothoracic

Surgeon, John Radcliffe

Hospital

Dr Matthew Hatton,

Consultant in Clinical

Oncology, Sheffield Teaching

Hospital Foundation Trust

Dr John Holden,

General Practitioner, Garswood

Surgery, Wigan
Dr Nadim Malik,

Consultant Cardiologist/

Honorary Lecturer, University

of Manchester

Mr Hisham Mehanna,

Consultant \& Honorary

Associate Professor, University

Hospitals Coventry \&

Warwickshire NHS Trust

Dr Jane Montgomery,

Consultant in Anaesthetics and

Critical Care, South Devon

Healthcare NHS Foundation

Trust

Dr Simon Padley,

Consultant Radiologist, Chelsea

\& Westminster Hospital
Dr Ashish Paul,

Medical Director, Bedfordshire PCT

Dr Sarah Purdy,

Consultant Senior Lecturer,

University of Bristol

Mr Michael Thomas,

Consultant Colorectal Surgeon, Bristol Royal Infirmary

Professor Yit Chiun Yang, Consultant Ophthalmologist,

Royal Wolverhampton Hospitals NHS Trust

Mrs Isabel Boyer,

Service User Representative,

London 


\section{Pharmaceuticals Panel}

Members

$\begin{array}{ll}\begin{array}{l}\text { Chair, } \\ \text { Professor Imti Choonara, } \\ \text { Professor in Child Health, } \\ \text { University of Nottingham }\end{array} & \begin{array}{l}\text { Dr Peter Elton, } \\ \text { Director of Public Health, } \\ \text { Bury Primary Care Trust }\end{array} \\ \begin{array}{l}\text { Deputy Chair, } \\ \text { Dr Lesley Wise, }\end{array} & \begin{array}{l}\text { Professor Robin Ferner, } \\ \text { Consultant Physician and }\end{array} \\ \text { Unit Manager, } & \begin{array}{l}\text { Director, West Midlands Centre } \\ \text { for Adverse Drug Reactions, }\end{array} \\ \text { Pharmacoepidemiology } & \text { City Hospital NHS Trust, } \\ \text { Research Unit, VRMM, } & \text { Birmingham } \\ \text { Medicines \& Healthcare } & \text { Dr Ben Goldacre, } \\ \text { Products Regulatory Agency } & \text { Research Fellow, Division of } \\ \text { Mrs Nicola Carey, } & \text { Psychological Medicine and } \\ \text { Senior Research Fellow, } & \text { Psychiatry, King's College } \\ \text { School of Health and Social } & \text { London } \\ \text { Care, The University of } & \text { Dr Bill Gutteridge, } \\ \text { Reading } & \text { Medical Adviser, London } \\ \text { Mr John Chapman, } & \text { Strategic Health Authority } \\ \text { Service User Representative } & \end{array}$

Dr Dyfrig Hughes,

Reader in Pharmacoeconomics and Deputy Director, Centre for Economics and Policy in Health, IMSCaR, Bangor University

Dr Yoon K Loke,

Senior Lecturer in Clinical Pharmacology, University of East Anglia

Professor Femi Oyebode, Consultant Psychiatrist and Head of Department, University of Birmingham

Dr Andrew Prentice,

Senior Lecturer and Consultant Obstetrician and Gynaecologist, The Rosie Hospital, University of Cambridge
Dr Martin Shelly,

General Practitioner, Leeds, and Associate Director, NHS Clinical Governance Support Team, Leicester

Dr Gillian Shepherd, Director, Health and Clinical Excellence, Merck Serono Ltd

Mrs Katrina Simister Assistant Director New Medicines, National Prescribing Centre, Liverpool

Mr David Symes,

Service User Representative

\section{Observers}

\section{Ms Kay Pattison}

Senior NIHR Programme

Manager, Department of

Health

Mr Simon Reeve,

Head of Clinical and Cost-

Effectiveness, Medicines,

Pharmacy and Industry Group,

Department of Health
Dr Heike Weber,

Programme Manager,

Medical Research Council
Dr Ursula Wells,

Principal Research Officer,

Department of Health

\section{Psychological and Community Therapies Panel}

Members

\section{Chair,}

Professor Scott Weich,

Professor of Psychiatry,

University of Warwick

Professor Jane Barlow, Professor of Public Health in the Early Years, Health Sciences Research Institute, Warwick Medical School

Dr Sabyasachi Bhaumik, Consultant Psychiatrist,

Leicestershire Partnership NHS Trust

Mrs Val Carlill,

Service User Representative, Gloucestershire

\section{Dr Steve Cunningham,}

Consultant Respiratory

Paediatrician, Lothian Health

Board

Dr Anne Hesketh,

Senior Clinical Lecturer in

Speech and Language Therapy,

University of Manchester

Dr Yann Lefeuvre,

GP Partner, Burrage Road

Surgery, London

Dr Jeremy J Murphy,

Consultant Physician \&

Cardiologist, County Durham \&

Darlington Foundation Trust

Mr John Needham,

Service User, Buckingmashire
Ms Mary Nettle,

Mental Health User Consultant,

Gloucestershire

Professor John Potter,

Professor of Ageing and Stroke Medicine, University of East

Anglia

Dr Greta Rait,

Senior Clinical Lecturer and General Practitioner, University College London

Dr Paul Ramchandani,

Senior Research Fellow/Cons

Child Psychiatrist, University of

Oxford

Dr Morven Roberts,

Clinical Trials Manager, MRC,

London
Professor Tom Walley,

HTA Programme Director,

Liverpool
Dr Howard Ring,

Consultant \& University

Lecturer in Psychiatry,

University of Cambridge

Dr Karen Roberts,

Nurse/Consultant, Dunston Hill Hospital, Tyne and Wear

Dr Karim Saad,

Consultant in Old Age

Psychiatry, Coventry \&

Warwickshire Partnership Trust

Dr Alastair Sutcliffe,

Senior Lecturer, University

College London

Dr Simon Wright,

GP Partner, Walkden Medical

Centre, Manchester
Senior NIHR Programme

Manager, Department of

Health 


\section{Expert Advisory Network}

\section{Members}

Professor Douglas Altman, Professor of Statistics in Medicine, Centre for Statistics in Medicine, University of Oxford

Professor John Bond, Professor of Social Gerontology \& Health Services Research, University of Newcastle upon Tyne

Professor Andrew Bradbury, Professor of Vascular Surgery, Solihull Hospital, Birmingham

Mr Shaun Brogan,

Chief Executive, Ridgeway Primary Care Group, Aylesbury

Mrs Stella Burnside OBE, Chief Executive, Regulation and Improvement Authority, Belfast

Ms Tracy Bury,

Project Manager, World

Confederation for Physical

Therapy, London

Professor Iain T Cameron, Professor of Obstetrics and Gynaecology and Head of the School of Medicine, University of Southampton

Dr Christine Clark,

Medical Writer and Consultant Pharmacist, Rossendale

Professor Collette Clifford, Professor of Nursing and Head of Research, The Medical School, University of Birmingham

Professor Barry Cookson, Director, Laboratory of Hospital Infection, Public Health Laboratory Service, London

Dr Carl Counsell,

Clinical Senior Lecturer in Neurology, University of Aberdeen

Professor Howard Cuckle, Professor of Reproductive Epidemiology, Department of Paediatrics, Obstetrics \& Gynaecology, University of Leeds

Dr Katherine Darton, Information Unit, MIND - The Mental Health Charity, London

Professor Carol Dezateux, Professor of Paediatric Epidemiology, Institute of Child Health, London

Mr John Dunning, Consultant Cardiothoracic Surgeon, Papworth Hospital NHS Trust, Cambridge
Mr Jonothan Earnshaw, Consultant Vascular Surgeon, Gloucestershire Royal Hospital, Gloucester

Professor Martin Eccles, Professor of Clinical Effectiveness, Centre for Health Services Research, University of Newcastle upon Tyne

Professor Pam Enderby, Dean of Faculty of Medicine, Institute of General Practice and Primary Care, University of Sheffield

Professor Gene Feder, Professor of Primary Care Research \& Development, Centre for Health Sciences, Barts and The London School of Medicine and Dentistry

Mr Leonard R Fenwick,

Chief Executive, Freeman Hospital, Newcastle upon Tyne

Mrs Gillian Fletcher, Antenatal Teacher and Tutor and President, National Childbirth Trust, Henfield

Professor Jayne Franklyn, Professor of Medicine, University of Birmingham

Mr Tam Fry

Honorary Chairman, Child Growth Foundation, London

Professor Fiona Gilbert, Consultant Radiologist and NCRN Member, University of Aberdeen

Professor Paul Grego Professor of Orthopaedic Surgical Science, South Tees Hospital NHS Trust

Bec Hanley,

Co-director, TwoCan Associates, West Sussex

Dr Maryann L Hardy, Senior Lecturer, University of Bradford

Mrs Sharon Hart,

Healthcare Management

Consultant, Reading

Professor Robert E Hawkins,

CRC Professor and Director

of Medical Oncology, Christie CRC Research Centre, Christie Hospital NHS Trust, Manchester

Professor Richard Hobbs, Head of Department of Primary Care \& General Practice, University of Birmingham

Professor Alan Horwich, Dean and Section Chairman,

The Institute of Cancer

Research, London
Professor Allen Hutchinson, Director of Public Health and Deputy Dean of ScHARR,

University of Sheffield

Professor Peter Jones, Professor of Psychiatry, University of Cambridge, Cambridge

Professor Stan Kaye

Cancer Research UK Professor of Medical Oncology, Royal Marsden Hospital and Institute of Cancer Research, Surrey

Dr Duncan Keeley,

General Practitioner (Dr Burch \& Ptnrs), The Health Centre, Thame

Dr Donna Lamping, Research Deorees Programme Director and Reader in Psychology, Health Services Research Unit, London School of Hygiene and Tropical Medicine, London

Mr George Levvy,

Chief Executive, Motor

Neurone Disease Association, Northampton

Professor James Lindesay, Professor of Psychiatry for the Elderly, University of Leicester

Professor Julian Little, Professor of Human Genome Epidemiology, University of Ottawa

Professor Alistaire McGuire, Professor of Health Economics, London School of Economics

Professor Rajan Madhok, Medical Director and Director of Public Health, Directorate of Clinical Strategy \& Public Health, North \& East Yorkshire \& Northern Lincolnshire Health Authority, York

Professor Alexander Markham, Director, Molecular Medicine Unit, St James's University Hospital, Leeds

Dr Peter Moore, Freelance Science Writer, Ashtead

Dr Andrew Mortimore, Public Health Director, Southampton City Primary Care Trust

Dr Sue Moss,

Associate Director, Cancer Screening Evaluation Unit, Institute of Cancer Research, Sutton
Professor Miranda Mugford, Professor of Health Economics and Group Co-ordinator, University of East Anglia

Professor Jim Neilson, Head of School of Reproductive \& Developmental Medicine and Professor of Obstetrics and Gynaecology, University of Liverpool

Mrs Julietta Patnick,

National Co-ordinator, NHS

Cancer Screening Programmes, Sheffield

Professor Robert Peveler, Professor of Liaison Psychiatry, Royal South Hants Hospital, Southampton

Professor Chris Price,

Director of Clinical Research, Bayer Diagnostics Europe, Stoke Poges

Professor William Rosenberg, Professor of Hepatology and Consultant Physician,

University of Southampton

Professor Peter Sandercock, Professor of Medical Neurology, Department of Clinical

Neurosciences, University of Edinburgh

Dr Susan Schonfield, Consultant in Public Health, Hillingdon Primary Care Trust, Middlesex

Dr Eamonn Sheridan,

Consultant in Clinical Genetics, St James's University Hospital, Leeds

Dr Margaret Somerville, Director of Public Health Learning, Peninsula Medical School, University of Plymouth

Professor Sarah Stewart-Brown, Professor of Public Health, Division of Health in the Community, University of Warwick, Coventry

Professor Ala Szczepura, Professor of Health Service Research, Centre for Health Services Studies, University of Warwick, Coventry

Mrs Joan Webster,

Consumer Member, Southern Derbyshire Community Health Council

Professor Martin Whittle, Clinical Co-director, National Co-ordinating Centre for Women's and Children's Health, Lymington 




\section{Feedback}

The HTA programme and the authors would like to know your views about this report.

The Correspondence Page on the HTA website (www.hta.ac.uk) is a convenient way to publish your comments. If you prefer, you can send your comments

to the address below, telling us whether you would like us to transfer them to the website.

\section{We look forward to hearing from you.}

NETSCC, Health Technology Assessment

Alpha House

University of Southampton Science Park

Southampton SOI6 7NS, UK

Email:hta@hta.ac.uk

www.hta.ac.uk 